\title{
Epidemiological and biological mechanisms of cannabis as cause of psychosis
}

Citation for published version (APA):

Küpper, R. (2012). Epidemiological and biological mechanisms of cannabis as cause of psychosis.

[Doctoral Thesis, Maastricht University]. Maastricht University. https://doi.org/10.26481/dis.20120504rk

Document status and date:

Published: 01/01/2012

DOI:

10.26481/dis.20120504rk

Document Version:

Publisher's PDF, also known as Version of record

\section{Please check the document version of this publication:}

- A submitted manuscript is the version of the article upon submission and before peer-review. There can be important differences between the submitted version and the official published version of record.

People interested in the research are advised to contact the author for the final version of the publication, or visit the DOI to the publisher's website.

- The final author version and the galley proof are versions of the publication after peer review.

- The final published version features the final layout of the paper including the volume, issue and page numbers.

Link to publication

\footnotetext{
General rights rights.

- You may freely distribute the URL identifying the publication in the public portal. please follow below link for the End User Agreement:

www.umlib.nl/taverne-license

Take down policy

If you believe that this document breaches copyright please contact us at:

repository@maastrichtuniversity.nl

providing details and we will investigate your claim.
}

Copyright and moral rights for the publications made accessible in the public portal are retained by the authors and/or other copyright owners and it is a condition of accessing publications that users recognise and abide by the legal requirements associated with these

- Users may download and print one copy of any publication from the public portal for the purpose of private study or research.

- You may not further distribute the material or use it for any profit-making activity or commercial gain

If the publication is distributed under the terms of Article $25 \mathrm{fa}$ of the Dutch Copyright Act, indicated by the "Taverne" license above, 
EPIDEMIOLOGICAL AND BIOLOGICAL MECHANISMS OF CANNABIS AS CAUSE OF PSYCHOSIS 
(C) Rebecca Küpper, Maastricht 2012

PRINT | Wöhrmann Print Service

ISBN | 978-90-8570-826-1 


\section{Epidemiological and biological mechanisms of cannabis as cause of psychosis}

\section{PROEFSCHRIFT}

ter verkrijging van de graad van doctor aan de Universiteit Maastricht, op gezag van de Rector Magnificus, Prof. Mr. G.P.M.F. Mols, volgens het besluit van het College van Decanen,

in het openbaar te verdedigen

op vrijdag 4 mei 2012 om 16:00 uur

door

\section{Rebecca Küpper}

Geboren op 9 oktober 1979 te Aken 


\section{Promotor}

Prof. dr. Jim van Os

\section{Co-promotor}

Dr. Cécile Henquet

\section{Beoordelingscommissie}

Prof. dr. Inez Myin-Germeys (voorzitter)

Prof. dr. Jan G. Ramaekers

Prof. dr. Don H. Linszen (Universiteit van Amsterdam, Amsterdam, The Netherlands)

Dr. Deepak C. D'Souza (Yale School of Medicine, New Haven, Connecticut, USA)

Dr. Ruud van Winkel

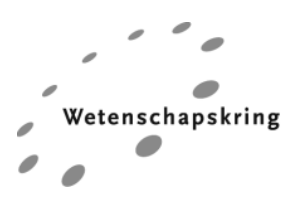

South Limburg Mental Health Research and Teaching Network PhD Series

The research presented in this thesis was conducted at the School for Mental Health and Neuroscience (MHeNS), Department of Psychiatry and Neuropsychology, Maastricht University, and Mondriaan Zorggroep Vijverdal. 


\section{Perspektiven}

Ob Lösung oder Trauma, Ein Doppel im Sinn macht Sinn.

Sei es verrückt oder verspielt, Die Deutung der Frage macht die Aussage.

Steh ich oben oder unten, Die Sicht macht die Aussicht.

Ist es fein oder klein, Der Stand macht den Punkt.

[Kai Savelsberg] 


\section{Paranimfen}

Anne-Kathrin Fett

Margreet Oorschot 


\section{CONTENTS}

CHAPTER 1

Introduction

\section{CHAPTER 2}

Cannabis use and risk of incidence and persistence of psychotic symptoms: 10 year follow-up cohort study

\section{CHAPTER 3}

Do cannabis and urbanicity co-participate in causing psychosis?

Evidence from a 10-year follow-up cohort study

\section{CHAPTER 4}

Non-replication of interaction between cannabis and trauma in predicting psychosis

\section{CHAPTER 5}

The dopamine dysfunction in psychosis revisited: New insights into topography and course

\section{CHAPTER 6}

Does dopamine mediate the psychosis-inducing effects of cannabis? A review and integration of findings across disciplines

\section{CHAPTER 7}

Delta-9-tetrahydrocannabinol-induced dopamine release as a function of psychotic disorder and psychotic vulnerability:

$\left[{ }^{18} \mathrm{~F}\right]$ fallypride PET study

\section{CHAPTER 8}

Is craving for cannabis increased in patients with psychotic disorder?

An Experience Sampling Study

\section{CHAPTER 9}

Does cannabidiol protect against the negative effects of THC?

\section{ChAPTER 10}

Discussion

Summary

Publications 

INTRODUCTION 
10 | INTRODUCTION 


\section{Phenomenology of psychosis}

Psychotic disorders, such as schizophrenia or other non-affective psychoses, are characterized by profound problems in the recognition of and interaction with reality [1]. Among the core symptoms are delusions and hallucinations, commonly referred to as the positive symptom cluster, and flattened affect, lack of motivation and anhedonia, also called the negative symptom dimension of psychosis [2]. Also, depressive mood, manic features and disorganized behavior are common in psychosis. According to the $4^{\text {th }}$ version of the Diagnostic and Statistical Manual of Mental Disorders [2], a diagnosis of schizophrenia or other psychotic disorder is fulfilled when symptoms occur independent of substance use or other medical conditions, are present a significant portion of time during one month, and interfere with social and occupational activities. Although not included in the classification of the DSM IV, psychotic disorders are further often accompanied by impairments in neurocognitive and social cognitive domains, including memory, executive functioning and theory of mind [3-5]. Some of the neurocognitive disturbances have been shown to strongly correlate with the negative and disorganized symptom clusters, yet not with the positive and depressive symptom dimensions [6], suggesting the existence of different cerebral mechanisms underlying the different symptom clusters. This is also in line with the proposed distinction between positive syndrome type I schizophrenia and negative syndrome type II schizophrenia [7-9]. While the former subtype is thought to be more common in females and characterized by mostly positive and affective symptoms, a better outcome, and a rather episodic and recurrent course, the latter subtype appears to be more common in males and has been associated with negative and cognitive symptoms, a worse outcome, and a rather deteriorating course [7-9]. Still, the distinction into two separable subtypes of schizophrenia remains a matter of debate. Notably, although neurocognition has been shown to correlate with certain symptom dimension, it does not seem to be a good predictor of functional outcome in psychotic disorders, which has been shown to more strongly correlate with measures of social cognition, in particular theory of mind [10].

The expression of psychosis does not appear to be restricted to clinically relevant psychotic disorders. Qualitatively similar psychotic experiences have been shown to be distributed in the general population [1 1-14]. Therefore, a dimensional view of psychosis, in which developmental expression of subclinical psychotic experiences is distinguished from clinically relevant psychotic symptoms by quantitative differences in symptom expression, might constitute a more adequate approach in contrast to the classic categorical view of a discrete and qualitatively different illness $[15,16]$. In support of the dimensional approach of psychosis are studies demonstrating that psychotic experiences show continuity with psychotic disorders such as schizophrenia in terms of familial clustering and shared etiological factors [17-20]. In addition, 
given the fact that transient psychotic experiences may, under certain circumstances, become abnormally persistent, giving rise to clinical psychotic disorder [13, 21-24], psychotic experiences represent an important phenotype for the investigation of mechanisms by which environmental risk factors such as cannabis impact on psychosis risk [24]. Therefore, these so-called sub-clinical psychotic symptoms are targeted in the epidemiological studies presented in this thesis (chapters 2, 3, and 4).

\section{Etiology of psychosis}

The risk factors for psychotic disorders such as schizophrenia are diverse. Besides a strong genetic component - first-degree relatives of patients with schizophrenia have a ten times higher risk of developing the disease compared to the general population [25] - research has identified a number of environmental factors that impact on psychosis risk [26]. Among the most common environmental influences are growing up in an urban environment [27-29], migration and discrimination [30-32], social deprivation and isolation [33-35], abuse and victimization [36, 37], obstetric complications [38-40] and cannabis use [41]. The latter being the central topic of investigation in this thesis.

The development and expression of vulnerability to psychosis might best be understood in terms of interaction between genes and environment [42]. Unlike gene-environment correlation, where a certain genetic make-up predisposes an individual to the exposure to certain environmental influences, gene-environment interaction implies that the degree to which environmental risk factors impact on psychosis risk is dependent on the level of individual, pre-existing genetic vulnerability for psychosis $[43,44]$. Statistically speaking, interaction between genes and environment may either be additive or multiplicative (discussed in more detail in chapter 3) and it has been suggested that interaction on the additive scale is biologically more plausible, yielding information on the extent to which two causes biologically depend on each other or co-participate in disease causation [45]. In line with these considerations, synergistic interaction between genetic predisposition for psychosis and environmental risk factors in causing psychosis has been identified for urbanicity [28], prenatal exposure to infections [46], psychosocial stress [47], trauma [37] and cannabis use [48]. 


\section{Cannabis use as cause of psychosis}

After tobacco and alcohol, cannabis is the most widely used recreational drug in the world [49]. According to the European Monitoring Centre on Drugs and Drug Addiction (EMCDDA), about $22.5 \%$ of European adults aged 15 to 65 have used cannabis at least once in their life and more than $6 \%$ have used cannabis in the last year [50]. Cannabis use is particularly prominent among young adults, with lifetime prevalence rates of about $31.6 \%$ in 15-34 year olds [50], which is alarming given the fact that the adolescent, still maturing brain might be particularly vulnerable to the adverse effects of cannabis [51, 52]. Notably, the prevalence of cannabis use differs largely throughout Europe but seems to be independent of availability in the respective countries: In the Netherlands, where cannabis is legally sold and consumed in so-called 'coffeeshops', the prevalence is rather moderate $(22.6 \%)$, compared to high-prevalence countries, which include Denmark (38.6\%), Italy $(32.0 \%)$ and the United Kingdom (31.1\%) [50].

\section{Biological and psychological effects of cannabis}

Cannabis is derived from the female plant of Cannabis Sativa and most of the effects associated with its use are caused by delta-9-tetrahydrocannabinol (THC), the main psychoactive constituent of cannabis. In the human brain, THC mainly binds to cannabinoid-1-receptors $(C B 1)$, where it acts as a partial agonist [53]. $C B 1$ receptors, which are the primary binding site of endogenous cannabinoids (eCBs) are densely distributed in various regions of the brain, including hippocampus, basal ganglia, amygdala, prefrontal cortex and cerebellum [54]. Among the best characterized eCBs are anandamide ( $\mathrm{N}$-arachidonoylethanolamide) and 2-AG (2arachidonoylglycerol) $[55,56]$. Being synthesized in and released on demand from the postsynaptic neuron, $\mathrm{eCBs}$ act in a retrograde fashion and bind to $C B 1$ receptors located on neighboring presynaptic terminals [57]. Activation of $C B 1$ receptors inhibits neurotransmitter release and thereby modulates several neurotransmitter systems, including the dopamine system [58]. The endocannabinoid system thus plays an important role in the fine-tuning of synaptic neurotransmission and activation of this system by exogenous cannabinoids such as THC can lead to profound and longlasting changes in synaptic functioning [52]. Yet, the exact effects of THC on the brain remain unknown, in particular with regard to long-term effects on mental health. The acute, psychological effects of cannabis include relaxation, sociability and euphoria but also anxiety, panic attacks or paranoia [49, 59]. Furthermore, cannabis use has been shown to affect cognitive functioning, in particular mnemonic and executive functioning as well as psychomotor speed, both in the short-term and beyond the period of acute intoxication [60-62]. Whether the neurocognitive effects of chronic long-term use are reversible remains unclear, since some level of impairment might still be detectable in abstinent users [63]. Intriguingly, in patients 
with schizophrenia, cannabis use has been associated with superior neurocognitive functioning compared to non-using patients $[64,65]$. With regard to the effects of cannabis use on brain structure, there is accumulating evidence for cannabis-related changes in brain morphology of chronic users, such as ventricular enlargement, reduced hippocampal and amygdala volume, and structural abnormalities in the corpus callosum [66-70]. Some studies further suggest that these changes occur dosedependent and are related to early age of onset of use $[67,68,70]$. With regard to long-term effects of cannabis use on mental health, cannabis use has been particularly associated with an increased risk to develop psychotic symptoms and schizophrenia [41].

\section{Epidemiological studies of cannabis as cause of psychosis}

Early evidence for a role of cannabis use in the emergence of psychosis was provided by a number of experimental studies on the acute effects of cannabis which demonstrated its ability to induce paranoid delusions and visual and auditory hallucinations in healthy individuals [71-73]. Later experimental studies on the acute effects of THC on psychopathology and cognitive endophenotypes of psychosis have replicated these psychotomimetic effects of cannabis [74-76]. One of the first large epidemiological studies that investigated the link between cannabis use and psychosis was conducted in 1987 [77]. The researchers examined the association between cannabis use and the development of schizophrenia during a 15-year follow-up in 45570 Swedish conscripts and revealed a dose-response effect: Individuals, who had used cannabis on 11 to 50 occasions had a three-fold increased risk of developing schizophrenia compared to non-users and in individuals who had used cannabis on more than fifty occasions the risk was increased even sixfold [77]. A later re-analysis of the same cohort for reasons of methodological refinement similarly reported a strong dose-response relationship between cannabis use and later development of schizophrenia, which was not explained by use of other drugs [78]. The link between cannabis use and psychosis was subsequently investigated by numerous epidemiological studies. By now, meta-analytic work has confirmed cannabis use as a risk factor for any psychotic outcome, including psychotic symptoms, schizophrenia or schizophreniform disorder, independent of factors such as age, gender, socio-economic status, and use of other drugs [41, 7982]. Yet, a much discussed topic in the cannabis-psychosis debate remains the issue of self-medication or reverse causality [83-86]: Is the association between cannabis use and psychosis indeed causal, or do early psychotic experiences in fact prompt cannabis use as a means of self-medication?

\section{Neurobiological aspects of the association between cannabis and psychosis}

It has been suggested that the psychosis-inducing effects of cannabis are mediated by increased dopaminergic neurotransmission in striatal regions of the brain [87]. 
Dysregulation of the dopamine system, in particular hypoactivity in the mesocortical dopamine pathway along with hyperactivity in subcortical dopamine pathways, has been implicated in the emergence and experience of psychotic symptoms for many year [88]. However, experimental studies employing neurochemical imaging techniques to investigate cannabis-induced changes in striatal dopamine neurotransmission in healthy human volunteers produced inconsistent results: While one study found increased striatal dopamine release following the acute administration of THC [89], two other studies failed to detect such an effect $[90,91]$. Yet, no study has investigated striatal dopamine release in response to THC in individuals with psychotic disorder. More research is therefore needed in order to clarify the apparent inconsistencies and to investigate neurobiological effects of THC in at-risk populations such as patients with psychotic disorder or their first-degree relatives.

\section{Differential sensitivity to cannabis as cause of psychosis}

The use of cannabis is neither sufficient nor necessary to precipitate psychotic symptoms. Cannabis use thus most likely constitutes a component cause of psychotic disorder, interacting with other genetic and environmental risk factors in increasing the risk for psychotic outcomes [92]. Accordingly, an important goal in recent research has been to identify factors that moderate the cannabis-psychosis relationship, rendering some individuals particularly vulnerable to the psychosisinducing effects of cannabis. Patients with a diagnosis of psychotic disorder have been found to show increased sensitivity to the acute effects of cannabis on behavioral outcomes such as cognition and psychotic reactivity [93]. Recently it was furthermore demonstrated that patients as well as their siblings showed increased cerebral cortical sensitivity to cannabis in terms of stronger reductions in cortical thickness compared to healthy controls [94]. Increased sensitivity with regard to the long-term effects of cannabis exposure on psychotic outcomes has also been demonstrated in individuals with underlying psychosis vulnerability or high schizotypy $[48,95,96]$. Studies on genetic moderation of the cannabis-psychosis relationship have mostly focused on the gene, which codes for the enzyme COMT (Catechyl-OMethyl-Transferase, an enzyme that is involved in the degradation of dopamine in the PFC). A functional single nucleotide polymorphism (SNP) at codon 158, the COMT val ${ }^{158}$ met polymorphism, has been shown to modulate dopamine levels in the human PFC $[97,98]$. Caspi and colleagues [99] were the first to demonstrate COMT $\mathrm{val}^{158}$ met moderation of the effects of adolescent cannabis use on later psychotic outcome. The risk for adult schizophreniform disorder was highest among individuals with the $\mathrm{ral} / \mathrm{val}$ genotype and lowest for the met/met genotype. The risk was intermediate for individuals with the val/met genotype. Experimental [100] as well as observational [101] studies have since replicated the moderating role of COMT val158met genotype. Yet, this idea has been challenged by a non-replication by 
now [102]. Recently, two other SNPs situated in the schizophrenia candidate gene AKT 1 were identified in relation to increased sensitivity to the psychosis-inducing effects of cannabis [103]. Overall, research thus suggests that (genetic) predisposition for psychosis as well as genetic variation of specific functional SNPs contributes to differential sensitivity to cannabis. Environmental factors, on the other hand, that might impact on the pathway from cannabis to psychosis have received only little attention. Some studies have indicated that early exposure to trauma induces differential sensitivity to cannabis effects in terms of psychosis risk [104, 105].

\section{Clinical implications}

Cannabis use is extremely common in clinical populations [106, 107] and has been associated with poor compliance, higher relapse rates and worse outcome among patients with schizophrenia [107-110]. Accordingly, cannabis use is often a major concern in the treatment of these patients and has been targeted by intervention strategies, albeit with little success [ 1111,112$]$. Yet, our understanding of patterns of initiation and continuation of use in at-risk populations and patients with established psychotic disorder is limited [1 13]. Self-reported reasons for cannabis use in patients with schizophrenia include enhancement of positive affect, alleviation of negative affect and dysphoria as well as avoidance of isolation and getting 'high' [1 14, 115]. These reasons similarly seem to apply to healthy controls [1 15], and are supported by research findings showing that cannabis use is associated with decreased negative symptoms in patients with schizophrenia [1 16 ]. In addition, evidence from Experience Sampling research suggests that cannabis use indeed increases positive affect and decreases negative affect in individuals with schizotypal traits [117]. Recently, Henquet and colleagues [1 18 ] investigated reactivity to cannabis use in daily life in patients with schizophrenia and healthy controls. In line with previous research, the study revealed a mood-enhancing effect of cannabis in both groups. Yet, the effect was greater in the patient group, suggesting that patients with schizophrenia were more sensitive to the positive effects of cannabis on mood. In addition, cannabis use was followed by an increase in hallucinatory experiences in patients only, which is in line with experimental research showing increased vulnerability to the acute psychotogenic effects of THC in patients with schizophrenia [93]. Furthermore it was demonstrated that the mood-enhancing effects occurred acutely, while the effect on hallucinations emerged only sub-acutely [1 18$]$. The temporal dissociation of cannabis effects in daily life might help to explain why patients continue to use: Cannabis use might be mostly associated with its positive effects while its negative psychosis-inducing effects are obscured. On the contrary, Dekker and colleagues recently demonstrated that, both implicitly and explicitly, patients tend to have negative associations with cannabis use [119]. 
Recently it has been suggested for the first time that patients with psychotic disorder experience higher levels of craving compared to healthy controls, independent of frequency of cannabis use [120]. Yet, this study did not control for the effects of antipsychotic medication and there is evidence that craving is associated with type of antipsychotic treatment [121]. Still, increased craving might be particularly instrumental for continuation of use in individuals with psychotic disorder. Increasing our understanding of patterns of cannabis use in such vulnerable populations is thus of great clinical relevance, in particular for the development of new and effective treatment strategies.

\section{Aims of this thesis}

Taken together, (genetic) epidemiological as well as experimental research has identified cannabis use as a component cause of psychotic disorder. Yet, issues such as reverse causality, differential sensitivity and environment-environment interaction, as well as the biological basis of the cannabis-psychosis relationship remain in need for further investigation. The research presented in this thesis aimed at shedding light on these matters by combining epidemiological (chapters 2, 3 and 4), observational (chapter 8) and experimental imaging research techniques (chapter 7) to elucidate both epidemiological and biological mechanisms by which exposure to cannabis increases the risk of psychotic disorder:

In order to clarify the temporal association, including the issue of reverse causality, between cannabis use and the development of psychotic symptoms and to shed light on possible mechanisms underlying this association, chapter 2 tests the hypothesis that adolescent cannabis use increases risk for psychotic outcomes by impacting on incidence and persistence of sub-clinical psychotic experiences in the general population.

The link between cannabis use and psychotic outcomes can best be understood in terms of gene-environment and environment-environment interactions. However, little is known about the moderating role of other environmental risk factors. Therefore, chapters 3 and 4 investigate possible environmental determinants of differential sensitivity to the long-term psychosis-inducing effects of cannabis, in particular urbanicity and trauma.

The neurotransmitter dopamine plays an important role in the pathophysiology of psychosis and interacts with the endocannabinoid system. Both systems might be involved in the psychosis-inducing effects of cannabis. Therefore, in chapters 5 and $\mathbf{6}$, evidence for the involvement of the dopamine system in the pathophysiology of 
psychosis is reviewed and the interaction between the dopamine and the endocannabinoid system is discussed, presenting possible biological mechanisms by which cannabis might increase psychosis risk. In line with these considerations, we subsequently test the hypothesis that the use of cannabis increases psychosis risk partly by inducing striatal dopamine release (chapter $\mathbf{7}$ ).

In order to shed further light on patterns and mechanisms of cannabis use in psychosis, chapter 8 applied the Experience Sampling Method to elucidate the role of craving and its association with continuation of use in the daily lives of patients with psychotic disorder and healthy controls.

Finally, chapter 9 discusses cannabidiol, another constituent of cannabis, as a potential antipsychotic agent and emphasizes the need to take into account differences in potency when investigating the effects of cannabis in future studies. 


\section{References}

1. Lewis, D.A. and J.A. Lieberman, Catching up on schizophrenia: natural history and neurobiology. Neuron, 2000. 28(2): p. 325-34.

2. American Psychiatric Association, Diagnostic and statistical manual of mental disorders. 4 ed. 2000, Washington, D.C.: Author.

3. Heinrichs, R.W. and K.K. Zakzanis, Neurocognitive deficits in schizophrenia: A quantitative review of the evidence. Neuropsychology, 1998. 12: p. 426-445.

4. Nuechterlein, K.H., et al., Identification of separable cognitive factors in schizophrenia. Schizophr Res, 2004. 72(1): p. 29-39.

5. Green, M.F., et al., Approaching a consensus cognitive battery for clinical trials in schizophrenia: the NIMH-MATRICS conference to select cognitive domains and test criteria. Biol Psychiatry, 2004. 56(5): p. $301-7$.

6. Dominguez Mde, G., et al., Are psychotic psychopathology and neurocognition orthogonal? A systematic review of their associations. Psychol Bull, 2009. 135(1): p. 157-71.

7. Andreasen, N.C., Positive vs. negative schizophrenia: a critical evaluation. Schizophrenia Bulletin, 1985. 11 (3): p. 380-9.

8. Carpenter, W.T., Jr., D.W. Heinrichs, and A.M. Wagman, Deficit and nondeficit forms of schizophrenia: the concept. American Journal of Psychiatry, 1988. 145(5): p. 578-83.

9. Murray, R.M., et al., A neurodevelopmental approach to the classification of schizophrenia. Schizophrenia Bulletin, 1992. 18(2): p. 319-32.

10. Fett, A.K., et al., The relationship between neurocognition and social cognition with functional outcomes in schizophrenia: A meta-analysis. Neuroscience and Biobehavioral Reviews, 2010.

11. Stefanis, N.C., et al., Evidence that three dimensions of psychosis have a distribution in the general population. Psychol Med, 2002. 32(2): p. 347-58.

12. Verdoux, H. and J. van Os, Psychotic symptoms in non-clinical populations and the continuum of psychosis. Schizophr Res, 2002. 54(1-2): p. 59-65.

13. Hanssen, M., et al., The incidence and outcome of subclinical psychotic experiences in the general population. British Journal of Clinical Psychology, 2005. 44(P+ 2): p. 181-91.

14. Rossler, W., et al., Psychotic experiences in the general population: a twenty-year prospective community study. Schizophrenia Research, 2007. 92(1-3): p. 1-14.

15. Johns, L.C. and J. van Os, The continuity of psychotic experiences in the general population. Clinical Psychology Review, 2001. 21(8): p. $1125-41$.

16. van Os, J., et al., A systematic review and meta-analysis of the psychosis continuum: evidence for a psychosis proneness-persistence-impairment model of psychotic disorder. Psychological Medicine, 2009. 39(2): p. 179-95.

17. Hanssen, M., et al., Evidence for instrument and family-specific variation of subclinical psychosis dimensions in the general population. Journal of Abnormal Psychology, 2006. 115(1): p. 5-14.

18. Versmissen, D., et al., Evidence for a relationship between mentalising deficits and paranoia over the psychosis continuum. Schizophrenia Research, 2008. 99(1-3): p. 103-10.

19. Krabbendam, L., et al., Familial covariation of the subclinical psychosis phenotype and verbal fluency in the general population. Schizophr Res, 2005. 74(1): p. 37-41.

20. Lataster, T., et al., Does reactivity to stress cosegregate with subclinical psychosis? A general population twin study. Acta Psychiatr Scand, 2009. 11 (1): p. 45-53.

21. Dominguez, M.D., et al., Evidence That Onset of Clinical Psychosis Is an Outcome of Progressively More Persistent Subclinical Psychotic Experiences: An 8-Year Cohort Study. Schizophrenia Bulletin, 2009. 
22. Cougnard, A., et al., Does normal developmental expression of psychosis combine with environmental risk to cause persistence of psychosis? A psychosis proneness-persistence model. Psychological Medicine, 2007. 37(4): p. 513-27.

23. Spauwen, J., et al., Does urbanicity shift the population expression of psychosis? Journal of Psychiatric Research, 2004. 38(6): p. 613-8.

24. Kuepper, R., et al., Continued cannabis use and risk of incidence and persistence of psychotic symptoms: 10 year follow-up cohort study. British Medical Journal, 2011 . 342: p. d738.

25. Gottesmann, I.I., Schizophrenia genesis: The origins of madness. 1991, New York: WH Freeman.

26. van Os, J., et al., The schizophrenia envirome. Curr Opin Psychiatry, 2005. 18(2): p. 141-5.

27. Marcelis, M., N. Takei, and J. van Os, Urbanization and risk for schizophrenia: does the effect operate before or around the time of illness onset? Psychological Medicine, 1999. 29(5): p. 1197-203.

28. Krabbendam, L. and J. van Os, Schizophrenia and urbanicity: a major environmental influence-conditional on genetic risk. Schizophrenia Bulletin, 2005. 31 (4): p. 795-9.

29. Kelly, B.D., et al., Schizophrenia and the city: A review of literature and prospective study of psychosis and urbanicity in Ireland. Schizophr Res, 2010. 116(1): p. 75-89.

30. Veling, W., H.W. Hoek, and J.P. Mackenbach, Perceived discrimination and the risk of schizophrenia in ethnic minorities: a case-control study. Soc Psychiatry Psychiatr Epidemiol, 2008. 43(12): p. 953-9.

31. Morgan, C., et al., Migration, ethnicity, and psychosis: toward a sociodevelopmental model. Schizophrenia Bulletin, 2010. 36(4): p. 655-64.

32. Weiser, M., et al., Elaboration on immigration and risk for schizophrenia. Psychological Medicine, 2008. 38(8): p. $1113-9$.

33. Allardyce, J., et al., Social fragmentation, deprivation and urbanicity: relation to first-admission rates for psychoses. British Journal of Psychiatry, 2005. 187: p. 401-6.

34. van Os, J., et al., Neighbourhood variation in incidence of schizophrenia. Evidence for personenvironment interaction. British Journal of Psychiatry, 2000. 176: p. 243-8.

35. Zammit, S., et al., Individuals, schools, and neighborhood: a multilevel longitudinal study of variation in incidence of psychotic disorders. Archives of General Psychiatry, 2010. 67(9): p. 914-22.

36. Read, J., et al., Childhood trauma, psychosis and schizophrenia: a literature review with theoretical and clinical implications. Acta Psychiatrica Scandinavica, 2005. $112(5)$ : p. 330-50.

37. Spauwen, J., et al., Impact of psychological trauma on the development of psychotic symptoms: relationship with psychosis proneness. British Journal of Psychiatry, 2006. 188: p. 527-33.

38. Geddes, J.R. and S.M. Lawrie, Obstetric complications and schizophrenia: a meta-analysis. British Journal of Psychiatry, 1995. 167(6): p. 786-93.

39. Marcelis, M., et al., Obstetric complications and familial morbid risk of psychiatric disorders. American Journal of Medical Genetics, 1998. 81(1): p. 29-36.

40. Spauwen, J., et al., Early maternal stress and health behaviours and offspring expression of psychosis in adolescence. Acta Psychiatrica Scandincavica, 2004. 110(5): p. 356-64.

41. Moore, T.H., et al., Cannabis use and risk of psychotic or affective mental health outcomes: a systematic review. Lancet, 2007. 370(9584): p. 319-28.

42. van Os, J., G. Kenis, and B.P. Rutten, The environment and schizophrenia. Nature, 2010. 468(7321): p. 203-12.

43. van Os, J. and P. Sham, Gene-environment correlation and interaction in schizophrenia, in The Epidemiology of Schizophrenia, R.M. Murray, et al., Editors. 2002, Cambridge University Press: Cambridge.

44. Nuechterlein, K.H. and M.E. Dawson, A heuristic vulnerability/stress model of schizophrenic episodes. Schizophrenia Bulletin, 1984. 10(2): p. 300-12.

45. Darroch, J., Biologic synergism and parallelism. American Journal of Epidemiology, 1997. 145(7): p. 661-8. 
46. Clarke, M.C., et al., Evidence for an interaction between familial liability and prenatal exposure to infection in the causation of schizophrenia. American Journal of Psychiatry, 2009. 166(9): p. 1025 30.

47. van Winkel, R., N.C. Stefanis, and I. Myin-Germeys, Psychosocial stress and psychosis. A review of the neurobiological mechanisms and the evidence for gene-stress interaction. Schizophrenia Bulletin, 2008. 34(6): p. 1095-105.

48. Henquet, C., et al., Prospective cohort study of cannabis use, predisposition for psychosis, and psychotic symptoms in young people. British Medical Journal, 2005. 330(7481): p. 11.

49. Murray, R.M., et al., Cannabis, the mind and society: the hash realities. Nature Reviews Neuroscience, 2007. 8(11): p. 885-95.

50. EMCDDA, Annual report on the state of the drugs problem in Europe. Vol. November 2010. 2010, Lisbon: EMCDDA.

51. Bossong, M.G. and R.J. Niesink, Adolescent brain maturation, the endogenous cannabinoid system and the neurobiology of cannabis-induced schizophrenia. Progress in Neurobiology, 2010.

52. Rubino, T. and D. Parolaro, Long lasting consequences of cannabis exposure in adolescence. Mol Cell Endocrinol, 2008. 286(1-2 Suppl 1): p. S108-13.

53. Pertwee, R.G., The diverse $C B 1$ and $C B 2$ receptor pharmacology of three plant cannabinoids: delta9tetrahydrocannabinol, cannabidiol and delta9-tetrahydrocannabivarin. Br J Pharmacol, 2008. 153(2): p. 199-215.

54. Herkenham, M., et al., Cannabinoid receptor localization in brain. Proc Natl Acad Sci U S A, 1990. 87(5): p. 1932-6.

55. Mechoulam, R., et al., Identification of an endogenous 2-monoglyceride, present in canine gut, that binds to cannabinoid receptors. Biochem Pharmacol, 1995. 50(1): p. 83-90.

56. Devane, W.A., et al., Isolation and structure of a brain constituent that binds to the cannabinoid receptor. Science, 1992. 258(5090): p. 1946-9.

57. Hashimotodani, Y., T. Ohno-Shosaku, and M. Kano, Endocannabinoids and synaptic function in the CNS. The Neuroscientist, 2007. 13: p. 127-137.

58. Fernandez-Ruiz, J., M. Hernandez, and J.A. Ramos, Cannabinoid-dopamine interaction in the pathophysiology and treatment of CNS disorders. CNS Neurosci Ther, 2010. 16(3): p. e72-91.

59. Hall, W. and N. Solowii, Adverse effects of cannabis. Lancet, 1998. 352(9140): p. $1611-6$.

60. Lundqvist, T., Cognitive consequences of cannabis use: comparison with abuse of stimulants and heroin with regard to attention, memory and executive functions. Pharmacol Biochem Behav, 2005. 81(2): p. 319-30.

61. Ranganathan, M. and D.C. D'Souza, The acute effects of cannabinoids on memory in humans: a review. Psychopharmacology (Berl), 2006. 188(4): p. 425-44.

62. Solowii, N. and R. Battisti, The chronic effects of cannabis on memory in humans: a review. Curr Drug Abuse Rev, 2008. 1(1): p. 81-98.

63. Solowii, N., Do cognitive impairments recover following cessation of cannabis use? Life Sci, 1995. 56(23-24): p. $2119-26$.

64. Yucel, M., et al., The Impact of Cannabis Use on Cognitive Functioning in Patients With Schizophrenia: A Meta-analysis of Existing Findings and New Data in a First-Episode Sample. Schizophrenia Bulletin, 2010.

65. Rabin, R.A., K.K. Zakzanis, and T.P. George, The effects of cannabis use on neurocognition in schizophrenia: a meta-analysis. Schizophrenia Research, 2011. 128(1-3): p. 111-6.

66. Welch, K.A., et al., The Impact of Substance Use on Brain Structure in People at High Risk of Developing Schizophrenia. Schizophrenia Bulletin, 2010.

67. Yucel, M., et al., Regional brain abnormalities associated with long-term heavy cannabis use. Arch Gen Psychiatry, 2008. 65(6): p. 694-701. 
68. Arnone, D., et al., Corpus callosum damage in heavy marijuana use: preliminary evidence from diffusion tensor tractography and tract-based spatial statistics. Neuroimage, 2008. 41(3): p. 1067-74.

69. Matochik, J.A., et al., Altered brain tissue composition in heavy marijuana users. Drug Alcohol Depend, 2005. 77(1): p. 23-30.

70. Wilson, W., et al., Brain morphological changes and early marijuana use: a magnetic resonance and positron emission tomography study. J Addict Dis, 2000. 19(1): p. 1-22.

71. Ames, F., A clinical and metabolic study of acute intoxication with Cannabis sativa and its role in the model psychoses. J Ment Sci, 1958. 104(437): p. 972-99.

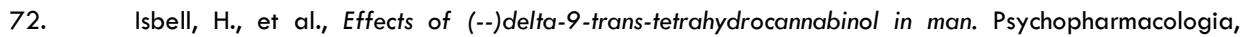
1967. $11(2):$ p. 184-8.

73. Talbott, J.A. and J.W. Teague, Marihuana psychosis. Acute toxic psychosis associated with the use of Cannabis derivatives. Jama, 1969. 210(2): p. 299-302.

74. D'Souza, D.C., et al., The psychotomimetic effects of intravenous delta-9-tetrahydrocannabinol in healthy individuals: implications for psychosis. Neuropsychopharmacology, 2004. 29(8): p. 1558-72.

75. Morrison, P.D., et al., The acute effects of synthetic intravenous Delta9-tetrahydrocannabinol on psychosis, mood and cognitive functioning. Psychol Med, 2009. 39(10): p. 1607-16.

76. Koethe, D., et al., Disturbances of visual information processing in early states of psychosis and experimental delta-9-tetrahydrocannabinol altered states of consciousness. Schizophrenia Research, 2006. 88(1-3): p. 142-50.

77. Andreasson, S., et al., Cannabis and schizophrenia. A longitudinal study of Swedish conscripts. Lancet, 1987. 2(8574): p. 1483-6.

78. Zammit, S., et al., Self reported cannabis use as a risk factor for schizophrenia in Swedish conscripts of 1969: historical cohort study. British Medical Journal, 2002. 325(7374): p. 1199.

79. Degenhardt, L. and W. Hall, Is cannabis use a contributory cause of psychosis? Can J Psychiatry, 2006. $51(9)$ : p. 556-65.

80. Henquet, C., et al., The environment and schizophrenia: the role of cannabis use. Schizophrenia Bulletin, 2005. 31(3): p. 608-12.

81. Semple, D.M., A.M. Mclntosh, and S.M. Lawrie, Cannabis as a risk factor for psychosis: systematic review. Journal of Psychopharmacology, 2005. 19(2): p. 187-94.

82. Arseneault, L., et al., Causal association between cannabis and psychosis: examination of the evidence. British Journal of Psychiatry, 2004. 184: p. 110-7.

83. Goswami, S., et al., Substance-abusing schizophrenics: do they self-medicate? American Journal of Addiction, 2004. 13(2): p. 139-50.

84. Ferdinand, R.F., et al., Cannabis use predicts future psychotic symptoms, and vice versa. Addiction, 2005. 100(5): p. $612-8$.

85. Degenhardt, L., W. Hall, and M. Lynskey, Testing hypotheses about the relationship between cannabis use and psychosis. Drug Alcohol Depend, 2003. 71(1): p. 37-48.

86. McGrath, J., et al., Association between cannabis use and psychosis-related outcomes using sibling pair analysis in a cohort of young adults. Archives of General Psychiatry, 2010. 67(5): p. 440-7.

87. Voruganti, L.N., et al., Cannabis induced dopamine release: an in-vivo SPECT study. Psychiatry Res, 2001. 107(3): p. 173-7.

88. Howes, O.D. and S. Kapur, The dopamine hypothesis of schizophrenia: version III--the final common pathway. Schizophrenia Bulletin, 2009. 35(3): p. 549-62.

89. Bossong, M.G., et al., Delta9-Tetrahydrocannabinol Induces Dopamine Release in the Human Striatum. Neuropsychopharmacology, 2008.

90. Stokes, P.R., et al., Can recreational doses of THC produce significant dopamine release in the human striatum? Neuroimage, 2009. 48(1): p. 186-90.

91. Barkus, E., et al., Does intravenous \{triangleup\}9-tetrahydrocannabinol increase dopamine release? $A$ SPET study. J Psychopharmacol, 2010. 
92. Henquet, C., et al., Gene-environment interplay between cannabis and psychosis. Schizophrenia Bulletin, 2008. 34(6): p. $1111-21$.

93. D'Souza, D.C., et al., Delta-9-tetrahydrocannabinol effects in schizophrenia: implications for cognition, psychosis, and addiction. Biological Psychiatry, 2005. 57(6): p. 594-608.

94. Habets, P., et al., Reduced cortical thickness as an outcome of differential sensitivity to environmental risks in schizophrenia. Biological Psychiatry, 2010. 69(5): p. 487-94.

95. Stirling, J., et al., Cannabis-induced psychotic-like experiences are predicted by high schizotypy. Confirmation of preliminary results in a large cohort. Psychopathology, 2008. 41(6): p. 371-8.

96. Barkus, E.J., et al., Cannabis-induced psychosis-like experiences are associated with high schizotypy. Psychopathology, 2006. 39(4): p. 175-8.

97. Chen, J., et al., Functional analysis of genetic variation in catechol-O-methyltransferase (COMT): effects on mRNA, protein, and enzyme activity in postmortem human brain. American Journal of Human Genetics, 2004. 75(5): p. 807-21.

98. Akil, M., et al., Catechol-O-methyltransferase genotype and dopamine regulation in the human brain. Journal of Neuroscience, 2003. 23(6): p. 2008-13.

99. Caspi, A., et al., Moderation of the effect of adolescent-onset cannabis use on adult psychosis by a functional polymorphism in the catechol-O-methyltransferase gene: longitudinal evidence of a gene $X$ environment interaction. Biological Psychiatry, 2005. 57(10): p. 1117-27.

100. Henquet, C., et al., An experimental study of catechol-o-methyltransferase Val158Met moderation of delta-9-tetrahydrocannabinol-induced effects on psychosis and cognition. Neuropsychopharmacology, 2006. 31 (12): p. 2748-57.

101. Henquet, C., et al., COMT ValMet moderation of cannabis-induced psychosis: a momentary assessment study of 'switching on' hallucinations in the flow of daily life. Acta Psychiatr Scand, 2009. 119(2): p. 156-60.

102. Zammit, S., et al., Cannabis, COMT and psychotic experiences. Br J Psychiatry, 2011.

103. van Winkel, R., Family-Based Analysis of Genetic Variation Underlying Psychosis-Inducing Effects of Cannabis: Sibling Analysis and Proband Follow-up. Archives of General Psychiatry, 201 1. 68(2): p. 148-57.

104. Harley, M., et al., Cannabis use and childhood trauma interact additively to increase the risk of psychotic symptoms in adolescence. Psychological Medicine, 2010. 40(10): p. 1627-34.

105. Houston, J.E., et al., Childhood sexual abuse, early cannabis use, and psychosis: testing an interaction model based on the National Comorbidity Survey. Schizophrenia Bulletin, 2008. 34(3): p. 580-5.

106. Green, B., R. Young, and D. Kavanagh, Cannabis use and misuse prevalence among people with psychosis. Br J Psychiatry, 2005. 187: p. 306-13.

107. Koskinen, J., et al., Rate of cannabis use disorders in clinical samples of patients with schizophrenia: a meta-analysis. Schizophrenia Bulletin, 2010. 36(6): p. $1115-30$.

108. Gregg, L., C. Barrowclough, and G. Haddock, Reasons for increased substance use in psychosis. Clinical Psychology Review, 2007. 27(4): p. 494-510.

109. Buhler, B., et al., Precipitation and determination of the onset and course of schizophrenia by substance abuse--a retrospective and prospective study of 232 population-based first illness episodes. Schizophrenia Research, 2002. 54(3): p. 243-51.

110. Zammit, S., et al., Effects of cannabis use on outcomes of psychotic disorders: systematic review. British Journal of Psychiatry, 2008. 193(5): p. 357-63.

111. Lobbana, F., et al., Understanding factors influencing substance use in people with recent onset psychosis: A qualitative study. Soc Sci Med, 2010. 70(8): p. $1141-7$.

112. Barrowclough, C., et al., Integrated motivational interviewing and cognitive behavioural therapy for people with psychosis and comorbid substance misuse: randomised controlled trial. British Medical Journal, 2010. 341: p. c6325.

113. Kolliakou, A., et al., Why do patients with psychosis use cannabis and are they ready to change their use? Int J Dev Neurosci, 2011 1. 29(3): p. 335-46. 


\section{$24 \mid$ INTRODUCTION}

114. Dekker, N., D.H. Linszen, and L. De Haan, Reasons for cannabis use and effects of cannabis use as reported by patients with psychotic disorders. Psychopathology, 2009. 42(6): p. 350-60.

115. Green, B., D.J. Kavanagh, and R.M. Young, Reasons for cannabis use in men with and without psychosis. Drug and Alcohol Review, 2004. 23(4): p. 445-53.

116. Potvin, S., A.A. Sepehry, and E. Stip, A meta-analysis of negative symptoms in dual diagnosis schizophrenia. Psychological Medicine, 2006. 36(4): p. 431-40.

117. Verdoux, H., et al., Effects of cannabis and psychosis vulnerability in daily life: an experience sampling test study. Psychological Medicine, 2003. 33(1): p. 23-32.

118. Henquet, C., et al., Psychosis reactivity to cannabis use in daily life: an experience sampling study. Br J Psychiatry, 2010. 196(6): p. 447-53.

119. Dekker, N., et al., Implicit and explicit affective associations towards cannabis use in patients with recent-onset schizophrenia and healthy controls. Psychol Med, 2010. 40(8): p. 1325-36.

120. Dekker, N., et al., Craving for cannabis in patients with psychotic disorderd, their non-affected siblings and healthy controls: psychometric analysis of the Obsessive Compulsive Drug Use Scale. International Journal of Methods in Psychiatric Research, in press.

121. Machielsen, M., et al., Differences in craving for cannabis between schizophrenia patients using risperidone, olanzapine or clozapine. J Psychopharmacol, 2011. 
\begin{tabular}{l|l} 
CHAPTER 1 & 25
\end{tabular} 



\title{
CONTINUED CANNABIS USE AND RISK OF INCIDENCE AND PERSISTENCE OF PSYCHOTIC SYMPTOMS: 10-YEAR FOLLOW-UP COHORT STUDY
}

\author{
Rebecca Kuepper ${ }^{1}$, Jim van Os1, 3, Roselind Lieb4, Hans-Ulrich \\ Wittchen ${ }^{5}$, Michael Hoefler ${ }^{5}$ \& Cécile Henquet $^{1,2}$
}

1 Department of Psychiatry and Neuropsychology, South Limburg Mental Health Research and Teaching Network, EURON, Maastricht University Medical Center, PO Box 616 (location

Vijverdal), NL-6200 MD, Maastricht, The Netherlands

2 PsyQ Heerlen, Mondriaan, South Limburg, The Netherlands

${ }^{3}$ King's College London, King's Health Partners, Department of Psychosis Studies, Institute of Psychiatry, London, United Kingdom

${ }^{4}$ Institute of Psychology, University of Basel, Birmannsgasse 8, CH-4009 Basel, Switzerland

5 Institute of Clinical Psychology and Psychotherapy, Technical University Dresden, Chemnitzerstrasse 46, D-01187 Dresden, Germany

British Medical Journal, 201 1. 342: p. d738. 


\section{Abstract}

Objective To test the hypothesis that adolescent cannabis use increases risk for psychotic outcomes by impacting on incidence and persistence of sub-threshold expression of psychosis in the general population.

Design Analysis of data from a prospective population-based cohort study in Germany (Early Developmental Stages of Psychopathology study).

Participants 1923 individuals from the general population (aged 14-24 years at baseline).

Main outcome measure Incidence and persistence of sub-threshold psychotic symptoms following adolescent cannabis use. Cannabis use and psychotic symptoms were assessed three times (baseline, T2, T3) over a 10-year follow-up period using the Munich version of the Composite International Diagnostic Interview (M-CIDI).

Results In individuals who were negative for lifetime psychotic symptoms and lifetime cannabis use at baseline, incident cannabis use over the baseline-T2 period increased the risk of later incident psychotic symptoms over the T2-T3 period (adjusted odds ratio $1.85,95 \%$ confidence interval 1.10 to $3.13, \mathrm{P}=0.021$ ). Furthermore, continued use of cannabis increased the risk of persistence of psychotic symptoms over the T2-T3 period (adjusted odds ratio $2.20,95 \%$ confidence interval 1.16 to $4.17, \mathrm{P}=0.016)$. The incidence rate of psychotic symptoms over the baseline-T2 period was $31 \%$ in exposed versus $20 \%$ in non-exposed individuals; over the period T2-T3 these rates were $14 \%$ and $8 \%$, respectively.

Conclusion Cannabis use is a risk factor for the development of incident psychotic symptoms. Furthermore, the data suggest that continued cannabis use increases the risk for psychotic disorder by impacting on the persistence of psychotic symptoms. 


\section{Introduction}

Cannabis is the most frequently used illicit drug in the world, particularly in adolescents [1,2]. The use of cannabis is consistently associated with mental illness [3], in particular psychotic disorder [4-9]. It remains a matter of debate, however, whether the association between cannabis and psychosis is causal, or whether early psychotic experiences may in fact prompt cannabis use as a means of selfmedication [10, 11]. This issue can only be resolved if incident cannabis use is investigated in relation to later incident psychotic symptoms or disorder. Rarely have studies been able to examine the longitudinal relationship between cannabis use and psychosis in this fashion.

The issue of self-medication was addressed by Henquet and colleagues [6], using data from the German prospective EDSP cohort study [12, 13]. The authors investigated the association between cannabis use at baseline and subsequent development of psychotic symptoms at four-year follow-up, and reported that after adjustment for pre-existing psychotic symptoms, cannabis use at baseline still remained significantly associated with psychotic symptoms at four-year follow-up. There was no evidence for self-medication effects, as pre-existing psychotic symptoms did not significantly predict later cannabis use [6]. Ferdinand and coworkers [11] investigated the role of pre-existing self-reported psychotic symptoms and demonstrated a bi-directional association between cannabis and psychotic symptoms over a 14-year follow-up study in the general population. The authors showed that cannabis use predicted later psychotic symptoms in individuals with no evidence of psychotic symptoms prior to onset of cannabis use and that the reverse was also true, in that psychotic symptoms predicted cannabis use in those who were cannabis naïve prior to onset of psychotic symptoms [11]. Evidence for a selfmedication effect was also demonstrated in a prospective population-based cohort study [14], in which it was found that individuals with self-reported hallucinations at the age of 14 years had a higher risk of using cannabis on a daily basis at 21 years. However, in a sibling pair analysis, this study also suggested an independent effect of cannabis use on self-reported delusional ideation later in life [14]. Thus, although the cannabis-psychosis link has been investigated in many studies, results on the temporal association between cannabis use and psychotic symptoms remain conflicting. Longitudinal cohort studies with multiple, repeated, interview-based measures of cannabis use and psychotic symptoms are needed to clarify this issue. The EDSP study $[12,13]$, which completed its recent 10-year follow-up, representing the fourth assessment (assessments at baseline, T1, T2 and T3, see also figure 1) is uniquely suitable for the renewed investigation of the temporal association between cannabis and psychosis. 
Another issue regards the mechanism by which cannabis may increase risk for psychotic symptoms, particularly the question whether cannabis may increase the risk for psychotic disorder by causing persistence of normally transitory developmental expression of psychotic experiences. For most individuals, sub-threshold psychotic phenomena are of a transitory nature and never progress to psychotic illness [15]. However, it has been shown that sub-threshold psychotic experiences may become abnormally persistent, depending on the degree of additional exposure to environmental risk factors [16-18], and that progressively greater levels of persistence are associated with greater risk for transition to clinical psychotic disorder [19]. Spauwen and colleagues [16] showed that the persistence rate of psychotic experiences was much higher for individuals growing up in an urban environment compared to those growing up in a rural environment [16]. Similarly, Cougnard and co-workers [17] provided evidence that childhood trauma, urbanicity and cannabis act additively in increasing the risk of persistence of psychotic experiences [17]. The fact that cannabis use increases psychosis risk in a doseresponse fashion $[6,14,20]$ and the finding that psychotic patients with continued cannabis use show more severe and persistent symptoms [21] suggests that cannabis use may increase the risk for psychotic illness by impacting on the persistence rate of psychotic experiences that under normal circumstances (i.e. without exposure to cannabis) would have remained transitory phenomena for the majority of individuals.

The aim of the present study, therefore, was to investigate, in a population-based 10-year follow-up cohort study of adolescents and young adults, the association between incident cannabis use and (i) true incidence of psychotic experiences (i.e. after exclusion of individuals with lifetime pre-existing psychotic experiences), and (ii) risk of persistence of psychotic experiences.

\section{Method}

\section{Sample and study design}

The observation frame was part of the Early Developmental Stages of Psychopathology (EDSP) study, which collected data on the prevalence, incidence, risk factors, comorbidity, and course of mental disorders in a random, representative population sample of adolescents and young adults in the general population [12, 13]. The baseline sample, following ethics committee approval, was randomly drawn, in 1994, from the respective population registry offices of Munich and its 29 counties, to mirror the distribution of individuals expected to be 14-24 years of age at the time of the baseline interview in 1995. The base population were all those born between June 1, 1970, and May 31, 1981, registered as residents in these 
localities and having German citizenship. These registers can be regarded as highly accurate because ( 1 ) each German is registered by his town, (2) they are regularly updated, (3) in the interest of scientific studies, any number of randomly drawn addresses with a given sex and age-group can be obtained, and (4) strict enforcement of registration by law and the police applies. More details on the sampling, representativeness, instruments, procedures, and statistical methods of the EDSP Study sample have been presented previously $[12,13]$.
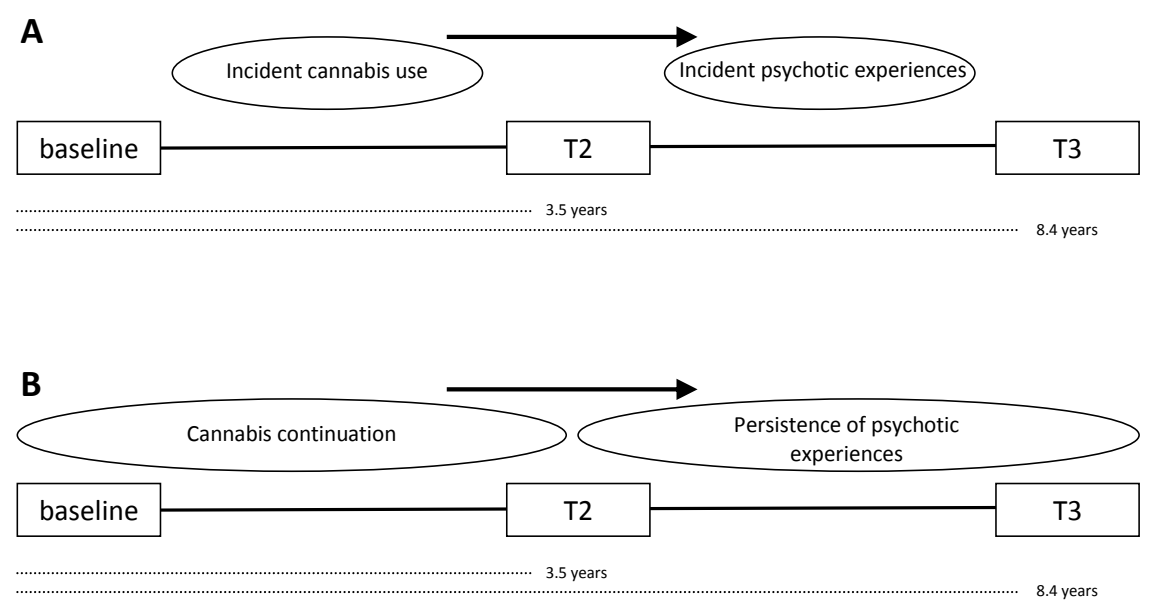

Figure 1. Study design.

A. Testing the association between incident cannabis use with onset in the period baseline-T2, and incident psychotic symptoms with onset in the period T2-T3, in individuals who were cannabis naïve at baseline and who had not reported any psychotic experience at T2 (i.e. no lifetime psychotic experiences by T2).

B. Testing the association between different cannabis exposure states (the combinations of cannabis use at baseline (lifetime) and/or T2 (interval) and persistence of psychotic experiences (i.e. presence of psychotic experiences at both T2 (lifetime) and T3 (interval)).

The overall design of EDSP is longitudinal and prospective, consisting of a baseline and 3 follow-up surveys, covering a time period of on average 1.6 years (baseline$\mathrm{T} 1, \mathrm{SD}=0.2$ ), 3.5 years (baseline-T2, SD $=0.3$ ), and 8.4 years (baseline-T3, range $=7.3-10.5 \mathrm{y}, \mathrm{SD}=0.7)$. Because the primary goal of the study was to examine the incidence and developmental risk factors for psychopathology, the younger group (14-15 y), presumed to have the highest incidence density, was sampled at twice the rate of persons aged 16-21 years, and the oldest group (2224 y) was sampled at half this rate. For the same reason, subjects aged 14-17 years at baseline were examined at the 4 time points and subjects aged 18-24 years were assessed only 3 times (baseline, T2, T3). The present study is based on the whole cohort assessed at baseline, T2 and T3. For a schematic illustration of the 
overall design and the current analyses see figure 1. Response rates were $84 \%$ at T2 and $74 \%$ at T3 (Fig. 2).

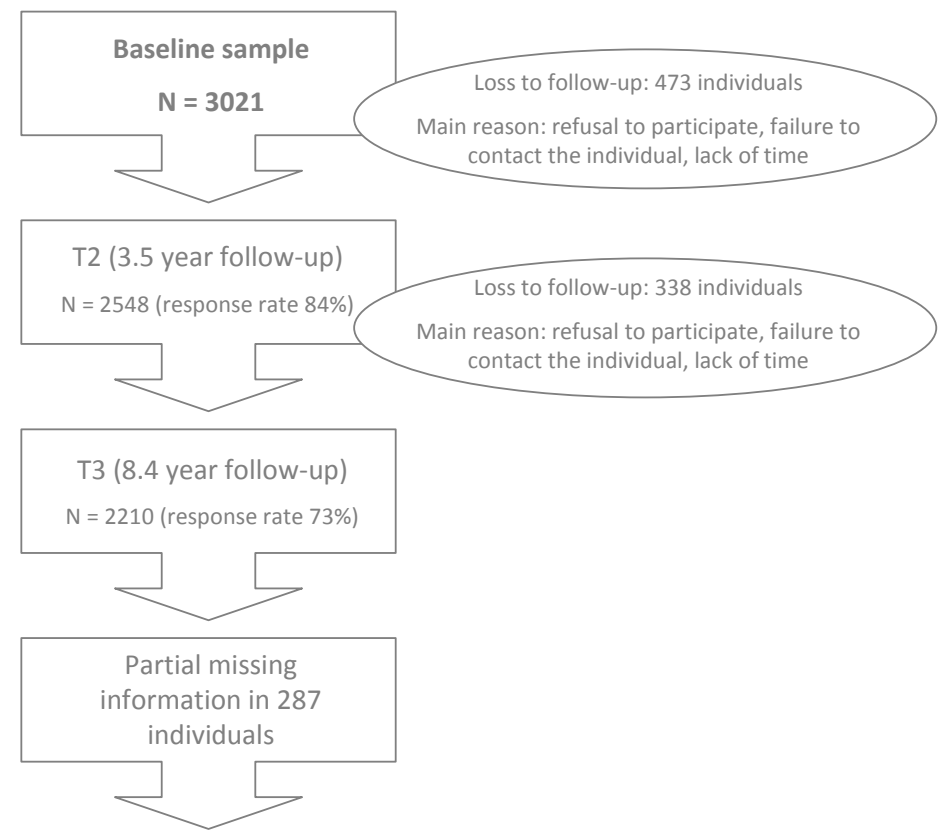

\section{Risk set $\mathrm{N}=1923$}

Figure 2. Schematic illustration of response rates and reasons for loss to follow-up.

\section{Instruments}

Participants were assessed using the computerized version of the Munich-Composite International Diagnostic Interview (DIA-X/M-CIDI) [22], an updated version of the World Health Organization's CIDI version 1.2. [23]. The DIA-X/M-CIDI is a comprehensive, fully standardized diagnostic interview and assesses symptoms, syndromes, and diagnoses of various mental disorders in accordance with the definitions and criteria of the International Classification of Diseases, Tenth Revision, and Diagnostic and Statistical Manual of Mental Disorders (Fourth Edition), along with information about onset, duration, severity of symptoms, and psychosocial impairment. The CIDI has been primarily designed for use in epidemiological studies of mental disorders and can also be used for clinical purposes. It is divided into 16 sections: 1 sociodemographic section, 12 sections assessing 288 symptoms of groups of mental disorders (including 'somatoform and dissociative,' 'phobic and other anxiety,' 'depressive and dysthymic,' 'manic and bipolar affective,' 'schizophrenia 
and other psychotic,' 'eating,' 'dementia and other cognitive,' 'posttraumatic stress,' as well as 'tobacco,' 'alcohol,' and 'substance-related' disorders), and 3 final sections containing concluding questions, interviewer observations, and interviewer ratings. The instrument, designed for use by trained interviewers who are not clinicians, has shown high inter-rater [24, 25] and test-retest reliability [22, 26]. However, the assessment of psychosis with CIDI by lay interviewers is not considered reliable. Therefore, in the EDSP Study, trained clinical interviewers at the level of clinical psychologist, who were allowed to probe with follow-up clinical questions, conducted the interviews in the respondents' homes. At baseline, the DIA-X/M-CIDI lifetime version was used. At each of the follow-up assessments, participants applied the interval version, which covers the period of assessment from the last interview until the next. Data on the $G$ section concerning psychosis and its clinical relevance were only collected at T2 (lifetime version) and T3 (interval version). Since the assessment of substance use was part of the diagnostic interview with the DIA-X/M-CIDI, psychologists who did the interviews were not blinded for cannabis use.

\section{Assessment of psychotic symptoms}

Data on positive psychotic experiences were collected at time T2 (lifetime version) and T3 (interval version) using the G-section of the DIA-X/M-CIDI. Since the primary objective of the EDSP study was to investigate the early stages of substance abuse in adolescents and young adults, data on the occurrence of psychotic symptoms as assessed with the G-section were added at T2 (measuring lifetime experience of psychotic symptoms) and T3 (measuring interval experience of psychotic symptoms) [13]. Conform previous work [6, 19] presence of positive psychotic experiences was broadly defined as any rating of 'present' on any of the 20 DIAX/M-CIDI core psychosis items (G1, G2a, G3-G5, G7-G13, G13b, G14, G17, G18, G20, G20C, G21, and G22a), including 14 delusion items, 5 hallucination items, and 1 item on passivity phenomena. Items relate to classic psychotic symptoms involving, e.g., persecution, thought interference, auditory hallucinations, and passivity phenomena. Participants were first invited by the psychologist to read a list of all the psychotic experiences and then asked whether they ever experienced such symptoms (list and phrasing available upon request). All these psychosis items were rated in 2 ways: 'absent' and 'present'. Thus, all the psychosis DIA-X/M-CIDI items used in the present study were coded in a dichotomous manner without intermediate levels. Psychotic symptoms at T2 accordingly referred to lifetime occurrence of minimally one positive rating on any psychosis item in the G-section; T3 psychotic symptoms denote interval occurrence of minimally one positive rating on any psychosis item in this section.

\section{Assessment of cannabis use}

Cannabis use was assessed with the L-section of the DIA-X/M-CIDI at all three assessments. The question 'Have you ever used cannabis more than five times' was 
used to define cannabis exposure. Consistent with previous work in this sample [6], the CIDI cut-off of use of five times or more was used to define cannabis exposure (yes versus no). Thus, at baseline, cannabis use was defined as lifetime use of cannabis of five times or more ('baseline cannabis use', dichotomously defined yes versus no). At T2, and similarly at $T 3$, cannabis use was defined as interval use of cannabis of five times or more, applied to the intervals of baseline-T2 and T2-T3, respectively ('T2 cannabis use', 'T3 cannabis use', both dichotomously defined yes versus no). As assessment of frequency of cannabis use differed between time points (i.e. at baseline: 'How often do you use cannabis on a five point scale ranging from less than monthly to almost daily?'; at T2: 'How many times have you used cannabis since the last assessment?'), the frequency variable was not included in the analyses.

\section{Statistical analyses}

Data were analyzed using STATA, release 10.0 (StataCorp, College Station, TX). Associations were expressed as odds ratios from logistic regression models.

All analyses were a priori adjusted for the following fixed and time-varying confounding factors: age at baseline (in years), gender $(0=$ female, $1=$ male), socioeconomic status (lower, middle, upper, other, as assessed at baseline), use of other drugs at baseline and T2, childhood trauma (i.e. trauma before the age of 14 years as assessed at baseline) [27] and urbanicity [28, 29]. The variable "use of other drugs' included psychostimulants, sedatives, opiates, cocaine, phencyclidine and psychedelic drugs and refers to CIDI-rated use of 5 times or more (lifetime or interval use at baseline and T2 respectively) of any of these drugs, as assessed with the L-section of the M-CIDI. 'Childhood trauma' refers to experience of any traumatic experience during childhood (i.e. before the age of 14 years) as assessed with the $\mathrm{N}$-section of the M-CIDI at baseline and was dichotomously defined as 'present' versus 'absent'. As demonstrated recently by Fisher and colleagues [30], self-report questionnaires constitute a valid method to assess childhood trauma in individuals presenting with psychotic features. Urbanicity was assessed at baseline by retrieving data on place of residence from the population registry offices of the city and the 29 counties of Munich, and was defined as either living in the city of Munich (4061 persons per square mile) or in the rural surroundings (553 persons per square mile) at baseline. To examine whether associations between cannabis use and psychotic symptoms were independent of other psychiatric diagnoses at baseline, analyses were additionally adjusted for any psychiatric diagnosis other than psychosis at baseline. A dichotomous variable 'any psychiatric diagnosis' (present versus absent) was calculated, summarizing main psychiatric diagnoses other than psychosis at baseline (i.e. major depressive disorder, hypomania, dysthymic disorder, bipolar disorder, panic disorder, obsessive compulsive disorder, posttraumatic stress disorder, conversion disorder, somatisation disorder, eating disorders). Sensitivity 
analyses were conducted to investigate whether attrition occurred at random and to assess potential bias introduced by missing data. This was done by multiple imputation of missing values using the ICE routine in Stata 11.1. This method imputes several alternative versions of the complete dataset using the data that is not missing.

Incident cannabis use and incidence of psychotic symptoms

Logistic regression analyses were used to investigate the association between baseline-T2 incident cannabis use and T2-T3 incident psychosis outcome (Fig. 1a). In order to investigate true incidence of psychotic symptoms following cannabis use, rather than persistence of pre-existing psychotic experiences, individuals who had admitted to lifetime presence of any psychotic symptom at T2 ( $\mathrm{n}=574,22.7 \%$ ) were excluded from the analysis. Similarly, all individuals with cannabis use at baseline were excluded, thus only including individuals with de novo cannabis exposure between baseline and T2. Reverse causality (i.e. self-medication) was investigated by testing the association between baseline-T2 psychosis and T2-T3 cannabis use in individuals without cannabis use at both baseline and T2.

Cannabis use and risk of persistence of psychotic symptoms

A dichotomous 'persistence' variable was calculated ('no persistence' versus 'persistence'), 'no persistence' referring to experience of psychotic symptoms either never or only once (at T2 or T3), and 'persistence' referring to to experience of psychotic symptoms at both T2 and T3. In order to investigate whether different levels of exposure to cannabis affected persistence of psychotic symptoms differentially, a categorical 'cannabis continuation' variable $0=$ never cannabis use; $1=$ cannabis use at baseline but not at T2; 2 = cannabis use at T2 but not at baseline, 3 = cannabis use at both baseline and T2) was calculated. Logistic regression analyses were used to investigate the association between cannabis continuation and psychosis persistence (Fig. 1b).

\section{Results}

\section{Sample}

A total of 2210 individuals completed the T3 assessment. 287 participants had partial missing information on substance use and psychotic symptoms, resulting in a final risk set for analysis of 1923 individuals of which 926 (48\%) were men (Figure 2). Mean age was 18.3 years (SD $=3.3$ years) at baseline, 21.8 (SD $=3.4$ years) at $\mathrm{T} 2$ and $26.6(\mathrm{SD}=3.5$ years) at T3. Further participant characteristics of the risk set are summarized in table 1 . 
Table 1. Participant characteristics per T2 cannabis usage group in the risk set $(N=1923)$. Figures are numbers (percentages organized by column).

\begin{tabular}{|c|c|c|}
\hline Gender & T2 cannabis use & no T2 cannabis use \\
\hline Male & $119(30.3 \%)$ & $807(52.7 \%)$ \\
\hline Female & $274(69.7 \%)$ & $723(47.3 \%)$ \\
\hline \multicolumn{3}{|l|}{ Socio-economic status* } \\
\hline Lower & $30(7.3 \%)$ & $85(5.6 \%)$ \\
\hline Middle & $220(53.8 \%)$ & $894(59.2 \%)$ \\
\hline Upper & $137(33.5 \%)$ & $524(34.7 \%)$ \\
\hline Other & $22(5.4 \%)$ & $6(0.4 \%)$ \\
\hline \multicolumn{3}{|l|}{ Urbanicity } \\
\hline Urban & $295(75.1 \%)$ & $1050(68.6 \%)$ \\
\hline Rural & $98(24.9 \%)$ & $480(31.4 \%)$ \\
\hline \multicolumn{3}{|l|}{ Childhood trauma } \\
\hline Present & $97(24.7 \%)$ & $266(17.4 \%)$ \\
\hline Absent & $296(75.3 \%)$ & $1264(82.6 \%)$ \\
\hline \multicolumn{3}{|l|}{$\begin{array}{l}\text { Use of other drugs } \\
\text { baseline }\end{array}$} \\
\hline Present & $25(6.4 \%)$ & $11(0.7 \%)$ \\
\hline Absent & $368(93.6 \%)$ & $1519(99.3 \%)$ \\
\hline \multicolumn{3}{|l|}{ Use of other drugs T2 } \\
\hline Present & $40(10.2 \%)$ & $3(0.2 \%)$ \\
\hline Absent & $353(89.8 \%)$ & $1527(99.8 \%)$ \\
\hline \multicolumn{3}{|l|}{$\begin{array}{l}\text { Any psychiatric disorder } \\
\text { baseline** }\end{array}$} \\
\hline Present & $76(19.3 \%)$ & $180(11.8 \%)$ \\
\hline Absent & $317(80.7 \%)$ & $1350(88.2 \%)$ \\
\hline
\end{tabular}

Note. Socio-economic status: lower (lower class, lower middle class), middle (middle middle class), upper (higher middle class, upper class) other (none of the above or missing). Residence: urban (the city of Munich, 4061 persons per square mile), rural (the surroundings of Munich, 553 persons per square mile). Childhood trauma: any traumatic experience during childhood. Use of other drugs more than five times baseline (lifetime) and T2 (interval).

* SES in $\mathrm{N}=1918$ due to missing values of 5 participants.

** denotes any psychiatric disorder at baseline according to M-CIDI diagnoses other than psychosis.

At baseline, 247 participants (12.8\%, lifetime use) reported cannabis use. Of those, 56 participants $(22.7 \%)$ used cannabis almost every day, $69(27.9 \%)$ reported weekly use and $57(23.1 \%)$ used cannabis monthly; 65 participants $(26.3 \%)$ reported cannabis use at a frequency of less than monthly. At T2, 392 participants (20.4\%, interval use) reported cannabis use. Mean frequency of those who reported cannabis use at T2 was 130 times within the baseline-T2 period (minimum 5 and maximum 997 times). Psychotic symptoms were reported by 436 participants 
$(22.7 \%)$ at T2 (lifetime) and by 231 participants (12.0\%) at T3 (interval). Table 2 summarises patterns of cannabis use in relation to psychotic symptoms.

Table 2. Patterns of cannabis use in relation to psychotic symptoms in $N=1923$ (columns: numbers of participants and percentages*).

\begin{tabular}{|c|c|c|c|c|}
\hline \multirow[b]{2}{*}{ Cannabis use $^{2}$} & \multicolumn{4}{|c|}{ Psychotic symptoms at follow up' } \\
\hline & \multicolumn{2}{|c|}{ T2 } & \multicolumn{2}{|c|}{ T3 } \\
\hline & Yes & No & Yes & No \\
\hline \multicolumn{5}{|l|}{ baseline } \\
\hline Yes & $81(4.2 \%)$ & $166(8.6 \%)$ & $42(2.2 \%)$ & $205(10.7 \%)$ \\
\hline No & $355(18.4 \%)$ & $1.321(68.7 \%)$ & $189(9.8 \%)$ & $1.487(77.3 \%)$ \\
\hline \multicolumn{5}{|l|}{ T2 } \\
\hline Yes & $126(6.7 \%)$ & $267(13.9 \%)$ & $69(3.6 \%)$ & $324(16.8 \%)$ \\
\hline No & $310(16.1 \%)$ & $1.220(63.5 \%)$ & $162(8.4 \%)$ & $1.368(71.2 \%)$ \\
\hline
\end{tabular}

*Some percentages do not total 100 due to rounding.

'Any psychotic symptom lifetime (T2) and interval (T3) as assessed with the M-CIDI (G) section.

${ }^{2}$ Use of more than 5 times lifetime (baseline) and interval (T2) as assessed with the M-CIDI (L) section.

\section{Incident cannabis use and incidence of psychotic symptoms}

Lifetime cannabis use as assessed at T2 significantly increased the risk of psychotic experiences at $\mathrm{T} 3$ (adjusted odds ratio $=1.49,95 \%$ confidence interval 1.07 to 2.07, $\mathrm{P}=0.018$; tables 3 and 4). After excluding all individuals who had used cannabis at baseline and had reported psychotic experiences at T2, incident cannabis use over the baseline-T2 period similarly increased the risk for incident psychotic experiences between T2 and T3 (adjusted odds ratio $=1.85,95 \%$ confidence interval 1.10 to $3.13, P=0.021)$. Results were unchanged after additional adjustment for any psychiatric diagnosis other than psychosis at baseline (adjusted odds ratio $=1.86,95 \%$ confidence interval 1.10 to $3.13, \mathrm{P}=0.020$ ). There was no evidence for self-medication effects, as T2 psychotic experiences did not predict incident cannabis use between T2 and T3 (adjusted odds ratio $=0.82$, $95 \%$ confidence interval 0.57 to $1.17, \mathrm{P}=0.3$ ).

\section{Cannabis use and persistence of psychotic experiences}

Analyses revealed a significant association between continuation of cannabis use and risk of persistence of psychotic experiences (adjusted $X^{2}=16.22, P=0.001$; tables 4 and 5). After adjustment for age, gender, socio-economic status, use of other drugs at baseline and T2, childhood trauma and urbanicity, occasional cannabis use (cannabis use at either baseline or T2, exposure states 1 and 2) effect sizes were attenuated, with statistical significance only for the combination of cannabis use at both baseline and $\mathrm{T} 2$ (exposure state 3 ; adjusted odds ratio $=2.20$, 
$95 \%$ confidence interval 1.16 to $4.17 ; \mathrm{P}=0.016)$. Additional adjustment for any psychiatric diagnosis other than psychosis at baseline only occasioned a slight reduction in the association between cannabis use at both baseline and T2 and the risk for persistence of psychotic symptoms (adjusted odds ratio $=1.99,95 \%$ confidence interval 1.04 to $3.81, P=0.037$ ).

Table 3. Association between incident cannabis use and incident psychotic experiences (columns: odds ratios and $95 \%$ confidence intervals; $P$-values).

\begin{tabular}{|c|c|c|}
\hline \multirow[b]{2}{*}{ T2 Cannabis use } & \multicolumn{2}{|c|}{ Risk of psychotic experiences at T3 } \\
\hline & Unadjusted & Adjusted* \\
\hline Whole sample & $1.8(1.32$ to $2.44 ; 0.000)$ & $1.49(1.07$ to $2.07 ; 0.018)$ \\
\hline After exclusion ${ }^{1}$ & $2.06(1.26$ to $3.38 ; 0.004)$ & $1.85(1.10$ to $3.13 ; 0.021)$ \\
\hline
\end{tabular}

*Adjusted for age, gender, socio-economic status, use of other drugs, childhood trauma and urbanicity.

${ }^{1}$ Individuals with baseline cannabis use and pre-existing psychotic symptoms were excluded from the analyses.

Table 4. Course of psychotic experiences in relation to level of continued cannabis use (columns: numbers of participants and percentages).

\begin{tabular}{|c|c|c|c|}
\hline \multirow[b]{2}{*}{ Cannabis continuation ${ }^{1}$} & \multicolumn{3}{|c|}{ Psychotic experiences at follow-up } \\
\hline & none & at either $\mathrm{T} 2$ or $\mathrm{T} 3$ & at both T2 and T3 \\
\hline 0 & $1.071(75 \%)$ & $303(21 \%)$ & $64(4 \%)$ \\
\hline 1 & $59(64 \%)$ & $25(27 \%)$ & $8(9 \%)$ \\
\hline 2 & $144(60 \%)$ & $75(32 \%)$ & $19(8 \%)$ \\
\hline 3 & $90(58 \%)$ & $48(31 \%)$ & $17(11 \%)$ \\
\hline
\end{tabular}

${ }^{1} 0=$ no use (reference category), $1=$ cannabis use at baseline but not at T2, 2 = cannabis use at T2 but not at baseline, 3 = cannabis use at baseline and $\mathrm{T} 2$

Table 5. Association between cannabis exposure states and persistence of psychotic experiences (columns: odds ratios and $95 \%$ confidence intervals; $P$-values).

\begin{tabular}{|c|c|c|}
\hline \multirow[b]{2}{*}{ Cannabis continuation ${ }^{1}$} & \multicolumn{2}{|c|}{ Risk of persistence of psychotic experiences } \\
\hline & Unadjusted & Adjusted* \\
\hline 0 & 1 & 1 \\
\hline 1 & $2.04(0.95$ to $4.40 ; 0.068)$ & $2.07(0.92$ to $4.66 ; 0.078)$ \\
\hline 2 & $1.86(1.09$ to $3.17 ; 0.022)$ & $1.43(0.82$ to $2.49 ; 0.202)$ \\
\hline 3 & $2.64(1.51$ to $4.64 ; 0.001)$ & $2.20(1.16$ to $4.17 ; 0.016)$ \\
\hline
\end{tabular}

\footnotetext{
*Adjusted for age, gender, socio-economic status, use of other drugs baseline and T2, childhood trauma and urbanicity.

$10=$ no use (reference category), $1=$ cannabis use at baseline but not at T2, 2 = cannabis use at T2 but not at baseline, 3 = cannabis use at baseline and $\mathrm{T} 2$.
} 


\section{Missing data}

Imputation of 20 datasets with the ICE routine in Stata 11.1, which imputes multiple alternatives based on data that is not missing revealed that the association between continued cannabis use and the risk of persistence of psychotic symptoms remained significant (adjusted $X^{2}=16.65, P=0.001$ ). Again, the strongest association was found for exposure state 3 (i.e. cannabis use at both baseline and T2) with an adjusted odds ratio of 1.99 (95\% confidence interval 1.08 to $3.66, \mathrm{P}=0.028$ ).

\section{Discussion}

This 10-year follow up study showed that incident cannabis use significantly increased the risk of incident psychotic experiences in individuals. The association was independent of age, gender, socio-economic status, use of other drugs, urbanicity and childhood trauma; additional adjustment for other psychiatric diagnoses similarly did not change the results. There was no evidence for selfmedication effects, since psychotic experiences did not predict later cannabis use. The results thus aid in clarifying the temporal association between cannabis use and psychotic experiences by systematically addressing the issue of reverse causality, given that the long follow-up period allowed all individuals with sensitively assessed pre-existing psychotic experiences or pre-existing cannabis use to be excluded. In addition, cannabis use was confirmed as an environmental risk factor impacting on the risk of persistence of psychotic experiences (Fig. 3).

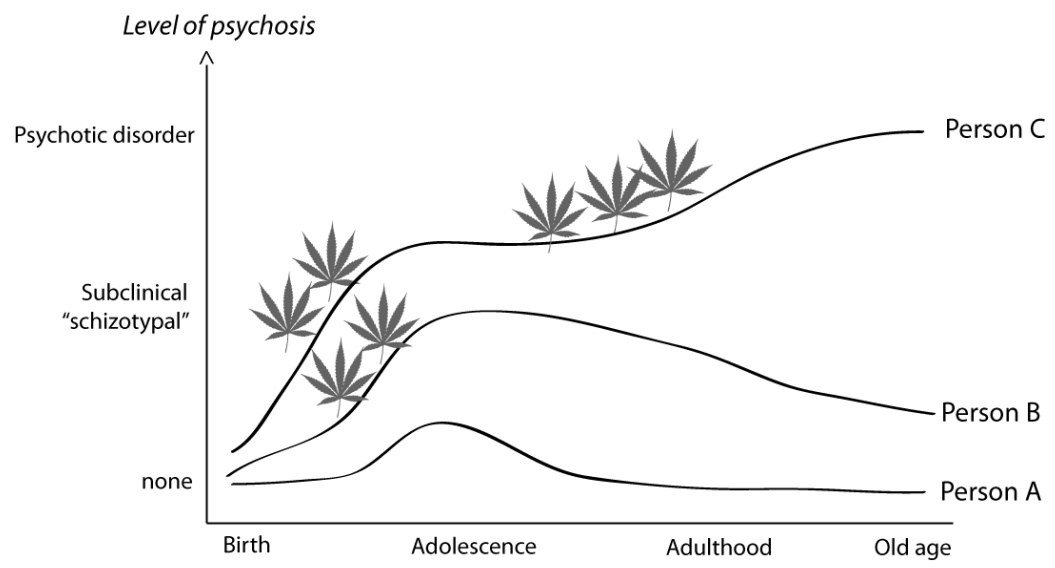

Figure 3. The cannabis-psychosis-persistence model.

Person A has "normal" developmental expression of sub-threshold psychotic experiences that are mild and transient. Person B has similar expression but longer persistence due to additional environmental exposure (here cannabis). Person $C$ has prolonged persistence and subsequent transition to clinical psychotic disorder due to repeated environmental exposure, i.e. repeated cannabis use. 


\section{Cannabis and psychosis: what is the mechanism behind the association?}

The present study investigated the association between cannabis use and psychosis risk by analyzing the expression of psychotic experiences. Psychotic experiences share many characteristics with clinically relevant psychosis, such as demographic, environmental and genetic risks, and are thought to represent a behavioural marker for psychosis liability [18, 31, 32]. It has been shown that psychotic experiences are a common and generally transitory phenomenon in the general population, that nevertheless may become abnormally persistent and progress to clinical psychotic disorder if combined with exposure to environmental risks [19,33,34]. The current study confirmed cannabis as an environmental risk factor, impacting on psychosis risk by (i) increasing the risk of incident psychotic experiences, and, if use continues over time, (ii) increasing the risk of persistence of psychotic experiences.

The finding that longer exposure to cannabis was associated with greater risk for persistence of psychotic experiences is in line with an earlier study showing that continued cannabis use over time increases the risk for psychosis in a dose-response fashion [5]. This is also in agreement with the hypothesis that a process of sensitization may underlie emergence and persistence of psychotic experiences [35] as an indicator of liability to psychotic disorder [18, 31]. Sensitization refers to the phenomenon that repeated exposure to an (environmental) stressor leads to progressively greater responses over time [35-38]. In rats, repeated exposure to THC (delta-9-tetrahydrocannabinol, the main psychoactive component of cannabis) induces behavioural sensitization: rats pre-treated with increasing doses of THC show greater behavioural (locomotor) responses to a THC challenge after a 14-day washout period than do THC-naïve rats [39, 40]. However, in humans, direct evidence for cannabis sensitization is lacking. Still, since the present study showed that the risk of persistence of psychotic experiences increases with longer periods of cannabis exposure, it is attractive to hypothesize that a process of sensitization underlies the association between cannabis and psychosis [32].

\section{Methodological issues}

The results should be interpreted in the light of several limitations. First, information on substance use and psychosis outcome was acquired using the DIA-X/M-CIDI, which essentially provides self-reported information. However, the interview was conducted face-to-face by clinical psychologists who were allowed to follow up with clinical questioning in order to ensure systematic and valid assessment of outcomes and can therefore be assumed to yield better and more valid results than a selfreport questionnaire. Secondly, the analyses were not directly adjusted for the possible confounding effects of a positive family history of psychosis, since this information was not available in the EDSP data. However, previous research has shown that associations between cannabis use and psychotic symptoms are not 
reducible to family history of psychosis [41, 42] and that genetic liability for psychotic disorder does not predict cannabis use [43]. In addition, individuals with a positive family history of psychosis report more positive symptoms than individuals without such predisposition [44, 45]. As all individuals with at least one T2 lifetime psychotic symptom were excluded from the analysis, the possible confounding effect of positive family history for psychosis was indirectly adjusted for to a degree. Further, we used a rather broad outcome measure, defined as a minimum of one positive rating on a $G$-section item, representing psychotic experiences rather than clinically relevant psychotic disorder. It has been shown however, that psychotic experiences show continuity with psychotic disorders such as schizophrenia $[18,46]$. In addition, given the fact that transient psychotic experiences may, under certain circumstances, become abnormally persistent, giving rise to clinical psychotic disorder $[15,17,19]$, psychotic experiences represent an important phenotype for the investigation of mechanisms and pathways by which environmental risk factors such as cannabis impact on psychosis risk. A further limitation concerns the use of the Gsection of the DIA-X/M-CIDI. This section was administered at T2 with a view to assess lifetime occurrence of symptoms which represents a long period for retrospective assessment of psychotic phenomena, possibly resulting in false negatives. As participants with T2 lifetime experience of psychotic symptoms were excluded from the analyses, underreporting would have resulted in false negatives being incorrectly kept in the analyses. It is unlikely however, that underreporting would have occurred as a function of cannabis use, which could have resulted in biased estimates. In addition, T2 lifetime subclinical psychotic symptoms were reported by $23 \%$, which is in keeping with the literature on the estimated $15-28 \%$ rate of subclinical psychotic symptoms in the general population [47]. Therefore, the influence of underreporting is likely limited. Finally, since the time between follow-up visits was 4 years on average, selective recall may have influenced the results. Spurious findings may have arisen if those with psychotic symptoms had better recall of earlier cannabis use. However, given the well-known link between psychosis liability and cognitive alterations including impaired memory, any influence of selective recall likely would have been conservative rather than anti-conservative. 


\section{References}

1. Wittchen, H.U., et al., A typology of cannabis-related problems among individuals with repeated illegal drug use in the first three decades of life: Evidence for heterogeneity and different treatment needs. Drug Alcohol Depend, 2009. 102(1-3): p. 151-7.

2. Perkonigg, A., et al., The natural course of cannabis use, abuse and dependence during the first decades of life. Addiction, 2008. 103(3): p. 439-49; discussion 450-1.

3. Wittchen, H.U., et al., Cannabis use and cannabis use disorders and their relationship to mental disorders: a 10-year prospective-longitudinal community study in adolescents. Drug and Alcohol Dependence, 2007. 88 Suppl 1: p. S60-70.

4. Moore, T.H., et al., Cannabis use and risk of psychotic or affective mental health outcomes: a systematic review. Lancet, 2007. 370(9584): p. 319-28.

5. van Os, J., et al., Cannabis use and psychosis: a longitudinal population-based study. American Journal of Epidemiology, 2002. 156(4): p. 319-27.

6. Henquet, C., et al., Prospective cohort study of cannabis use, predisposition for psychosis, and psychotic symptoms in young people. British Medical Journal, 2005. 330(7481): p. 11.

7. Arseneault, L., et al., Causal association between cannabis and psychosis: examination of the evidence. British Journal of Psychiatry, 2004. 184: p. $110-7$.

8. Semple, D.M., A.M. Mclntosh, and S.M. Lawrie, Cannabis as a risk factor for psychosis: systematic review. Journal of Psychopharmacology, 2005. 19(2): p. 187-94.

9. Degenhardt, L. and W. Hall, Is cannabis use a contributory cause of psychosis? Can J Psychiatry, 2006. 51 (9): p. 556-65.

10. Degenhardt, L., W. Hall, and M. Lynskey, Testing hypotheses about the relationship between cannabis use and psychosis. Drug Alcohol Depend, 2003. 71(1): p. 37-48.

11. Ferdinand, R.F., et al., Cannabis use predicts future psychotic symptoms, and vice versa. Addiction, 2005. 100(5): p. 612-8.

12. Lieb, R., et al., The Early Developmental Stages of Psychopathology Study (EDSP): a methodological update. European Addiction Research, 2000. 6(4): p. 170-82.

13. Wittchen, H.U., et al., Early developmental stages of psychopathology study (EDSP): objectives and design. European Addiction Research, 1998. 4(1-2): p. 18-27.

14. McGrath, J., et al., Association between cannabis use and psychosis-related outcomes using sibling pair analysis in a cohort of young adults. Archives of General Psychiatry, 2010. 67(5): p. 440-7.

15. Hanssen, M., et al., The incidence and outcome of subclinical psychotic experiences in the general population. British Journal of Clinical Psychology, 2005. 44(Pt 2): p. $181-91$.

16. Spauwen, J., et al., Evidence that the outcome of developmental expression of psychosis is worse for adolescents growing up in an urban environment. Psychological Medicine, 2006. 36(3): p. 407-15.

17. Cougnard, A., et al., Does normal developmental expression of psychosis combine with environmental risk to cause persistence of psychosis? A psychosis proneness-persistence model. Psychological Medicine, 2007. 37(4): p. 513-27.

18. van Os, J., et al., A systematic review and meta-analysis of the psychosis continuum: evidence for a psychosis proneness-persistence-impairment model of psychotic disorder. Psychological Medicine, 2009. 39(2): p. 179-95.

19. Dominguez, M.D., et al., Evidence That Onset of Clinical Psychosis is an Outcome of Progressively More Persistent Subclinical Psychotic Experiences: An 8-Year Cohort Study. Schizophrenia Bulletin, 2009.

20. Zammit, S., et al., Self reported cannabis use as a risk factor for schizophrenia in Swedish conscripts of 1969: historical cohort study. British Medical Journal, 2002. 325(7374): p. 1199.

21. Grech, A., et al., Cannabis use and outcome of recent onste psychosis. European Psychiatry, 2005. 20(4): p. 349-353. 
22. Wittchen, H.U., et al., Test-retest reliability of the computerized DSM-IV version of the MunichComposite International Diagnostic Interview (M-CIDI). Social Psychiatry and Psychiatric Epidemiology, 1998. 33(1 1): p. 568-78.

23. Robins, L.N., et al., The Composite International Diagnostic Interview. An epidemiologic Instrument suitable for use in conjunction with different diagnostic systems and in different cultures. Archives of General Psychiatry, 1988. 45(12): p. 1069-77.

24. Cottler, L.B., et al., Agreement between DSM-III and III-R substance use disorders. Drug and Alcohol Dependence, 1991. 29(1): p. 17-25.

25. Wittchen, H.U., et al., Cross-cultural feasibility, reliability and sources of variance of the Composite International Diagnostic Interview (CIDI). The Multicentre WHO/ADAMHA Field Trials. British Journal of Psychiatry, 1991. 159: p. 645-53, 658.

26. Wittchen, H.U., Reliability and validity studies of the WHO--Composite International Diagnostic Interview (CIDI): a critical review. Journal of Psychiatry Research, 1994. 28(1): p. 57-84.

27. Read, J., et al., Childhood trauma, psychosis and schizophrenia: a literature review with theoretical and clinical implications. Acta Psychiatrica Scandinavica, 2005. 112 (5): p. 330-50.

28. Krabbendam, L. and J. van Os, Schizophrenia and urbanicity: a major environmental influence-conditional on genetic risk. Schizophrenia Bulletin, 2005. 31 (4): p. 795-9.

29. Van Os, J., et al., The schizophrenia envirome. Current Opinion in Psychiatry, 2005. 18: p. $141-145$.

30. Fisher, H.L., et al., Reliability and Comparability of Psychosis Patients' Retrospective Reports of Childhood Abuse. Schizophr Bull, 2009.

31. Polanczyk, G., et al., Etiological and clinical features of childhood psychotic symptoms: results from a birth cohort. Archives of General Psychiatry, 2010. 67(4): p. 328-38.

32. Van Os, J., G. Kenis, and B. Rutten, The environment and schizophrenia. Nature, in press.

33. Rossler, W., et al., Psychotic experiences in the general population: a twenty-year prospective community study. Schizophrenia Research, 2007. 92(1-3): p. 1-14.

34. van Rossum, l., et al., Affective Dysregulation and Reality Distortion: A 10-Year Prospective Study of Their Association and Clinical Relevance. Schizophrenia Bulletin, 2009.

35. Collip, D., I. Myin-Germeys, and J. Van Os, Does the concept of "sensitization" provide a plausible mechanism for the putative link between the environment and schizophrenia? Schizophrenia Bulletin, 2008. 34(2): p. 220-5.

36. Myin-Germeys, I., P. Delespaul, and J. van Os, Behavioural sensitization to daily life stress in psychosis. Psychological Medicine, 2005. 35(5): p. 733-41.

37. O'Daly, O., et al., Schizophrenia and substance abuse co-morbidity: a role for dopamine sensitization? Journal of Dual Diagnosis, 2005. 1(2): p. 11-40.

38. Howes, O.D., et al., Pathways to schizophrenia: the impact of environmental factors. International Journal of Neuropsychopharmacology, 2004. 7 Suppl 1: p. S7-S13.

39. Cadoni, C., et al., Behavioural sensitization after repeated exposure to Delta 9tetrahydrocannabinol and cross-sensitization with morphine. Psychopharmacology (Berl), 2001. 158(3): p. 259-66.

40. Cadoni, C., V. Valentini, and G. Di Chiara, Behavioral sensitization to delta 9-tetrahydrocannabinol and cross-sensitization with morphine: differential changes in accumbal shell and core dopamine transmission. Journal of Neurochemistry, 2008. 106(4): p. 1586-93.

41. Andreasson, S., et al., Cannabis and schizophrenia. A longitudinal study of Swedish conscripts. Lancet, 1987. 2(8574): p. 1483-6.

42. van Os, J., et al., Cannabis use and psychosis: a longitudinal population-based study. Am J Epidemiol, 2002. 156(4): p. 319-27.

43. G.R.O.U.P, Evidence That Familial Liability for Psychosis Is Expressed as Differential Sensitivity to Cannabis: An Analysis of Patient-Sibling and Sibling-Control Pairs. Arch Gen Psychiatry, 2010. 
44. Keshavan, M., et al., Psychopathology among offspring of parents with schizophrenia: relationship to premorbid impairments. Schizophr Res, 2008. 103(1-3): p. 114-20.

45. Niemi, L.T., et al., Childhood developmental abnormalities in schizophrenia: evidence from high-risk studies. Schizophr Res, 2003. 60(2-3): p. 239-58.

46. Johns, L.C. and J. van Os, The continuity of psychotic experiences in the general population. Clinical Psychology Review, 2001. 21 (8): p. $1125-41$.

47. Verdoux, H. and J. van Os, Psychotic symptoms in non-clinical populations and the continuum of psychosis. Schizophr Res, 2002. 54(1-2): p. 59-65. 



\title{
DO CANNABIS AND URBANICITY CO-PARTICIPATE IN
}

\section{CAUSING PSYCHOSIS? EVIDENCE FROM A 10 -YEAR}

\section{FOLLOW-UP COHORT STUDY}

\author{
Rebecca Kuepper ${ }^{1}$, Jim van Os ${ }^{1}, 3$, Roselind Lieb ${ }^{4}$, Hans-Ulrich Wittchen ${ }^{5}$, \\ \& Cécile Henquet ${ }^{1,2}$
}

\footnotetext{
1 Department of Psychiatry and Neuropsychology, South Limburg Mental Health Research and Teaching Network, EURON, Maastricht University Medical Center, PO Box 616 (location Vijverdal), NL-6200 MD, Maastricht, The Netherlands

2 PsyQ Heerlen, Mondriaan, South Limburg, The Netherlands ${ }^{3}$ King's College London, King's Health Partners, Department of Psychosis Studies, Institute of Psychiatry, London, United Kingdom

${ }^{4}$ Institute of Psychology, University of Basel, Birmannsgasse 8, CH-4009 Basel, Switzerland 5 Institute of Clinical Psychology and Psychotherapy, Technical University Dresden, Chemnitzerstrasse 46, D-01187 Dresden, Germany
}

Psychological Medicine, 2011.41(10): p. 2121-2129. 


\section{Abstract}

Background Cannabis use is considered a component cause of psychotic illness, interacting with genetic and other environmental risk factors. Little is known, however, about these putative interactions. The present study investigated whether an urban environment plays a role in moderating the effects of adolescent cannabis use on psychosis risk.

Methods Prospective data ( $N=1923$, aged 14-24 years at baseline) from the longitudinal population-based German Early Developmental Stages of Psychopathology (EDSP) cohort study were analysed. Urbanicity was assessed at baseline and defined as living in the city of Munich (4061 individuals per square mile) or in the rural surroundings (553 individuals per square mile). Cannabis use and psychotic symptoms were assessed three times over a 10-year follow-up period using the Munich version of the Composite International Diagnostic Interview (M-CIDI). Results Analyses revealed a significant interaction between cannabis and urbanicity (10.9\% adjusted difference in risk, $95 \% \mathrm{Cl}$ : 3.2-18.6, $\mathrm{p}=0.005)$. The effect of cannabis use on follow-up incident psychotic symptoms was much stronger in individuals who grew up in an urban environment (adjusted risk difference $6.8 \%$, $95 \% \mathrm{Cl}: 1-12.5, \mathrm{p}=0.021$ ) compared to individuals from rural surroundings (adjusted risk difference $-4.1 \%, 95 \% \mathrm{Cl}$ : $-9.8-1.6, \mathrm{p}=0.159$ ). The statistical interaction was compatible with substantial underlying biological synergism.

Conclusions Exposure to environmental influences associated with urban upbringing may increase vulnerability to the psychotomimetic effects of cannabis use later in life. 


\section{Introduction}

Adolescent cannabis use may increase the risk of psychotic disorder [1-3], the first stage of which is expressed as abnormal persistence of subclinical psychotic symptoms $[4,5]$. However, only a minority of cannabis users will eventually develop a psychotic disorder and many individuals with psychotic illness have never been exposed. Therefore, cannabis use likely constitutes a component cause, codepending on other causal influences in shaping risk for psychosis [6]. Little is known, however, about the nature of these putative interactions $[1,7,8]$.

Using data from the prospective German Early Developmental Stages of Psychopathology study [9, 10], Henquet and colleagues [1 1] showed that individuals scoring high on schizotypy had a much higher risk of developing psychotic symptoms after cannabis use compared to individuals with low or average schizotypy scores [11]. A subsequent study suggested that the moderating effect of schizotypy on the psychotomimetic effect of cannabis use may be mediated by genetic risk for psychotic disorder [12] with some evidence for specific molecular genetic variation $[8,13,14]$. Environmental factors may similarly moderate the long term effects of cannabis on psychosis outcomes, as both Houston and colleagues [15] and Harley and colleagues [16] reported that individuals who were exposed to trauma early in life, compared to those without trauma, had a much higher risk to develop psychotic outcomes following adolescent cannabis use. Thus, the risk conveyed by the combination of cannabis and trauma was much higher than the sum of the risk posed by either factor alone $[15,16]$.

Other environmental risk factors may also interact with cannabis use. For example, there is evidence that cannabis use, childhood trauma and growing up in an urban environment independently increase the risk of onset and persistence of psychotic symptoms [4] and it has been hypothesized that all three may be associated with the same underlying mechanism of behavioural 'sensitisation' over time [17-19]. Metaanalyses suggest that growing up in an urban environment is consistently associated, in a dose-response fashion, with increased psychosis risk [20, 21], particularly if there is additional evidence of genetic risk [22-24]. The aim of the present study was to investigate whether environmental factors associated with urbanicity moderate the strength of the cannabis-psychosis relationship. Data from the German Early Developmental Stages of Psychopathology (EDSP) study [9, 10] were analyzed to investigate interaction between cannabis use and urbanicity on later expression of psychotic symptoms in adolescents and young adults. 


\section{Method}

\section{Sample and study design}

The Early Developmental Stages of Psychopathology (EDSP) study collected data on the prevalence, incidence, risk factors, comorbidity, and course of mental disorders in a random, representative population sample of adolescents and young adults in the general population [9, 10]. The baseline sample, following ethics committee approval, was randomly drawn, in 1994, from the respective population registry offices of Munich and its 29 counties, to mirror the distribution of individuals expected to be 14-24 years of age at the time of the baseline (TO) interview in 1995. The base population were all those born between June 1, 1970, and May 31,1981 , registered as residents in these localities and having German citizenship. These registers can be regarded as highly accurate because (1) each German is registered by his town, (2) they are regularly updated, (3) in the interest of scientific studies, any number of randomly drawn addresses with a given sex and age-group can be obtained, and (4) strict enforcement of registration by law and the police applies. More details on the sampling, representativeness, instruments, procedures, and statistical methods of the EDSP Study sample have been presented previously $[9,10]$.

The overall design of the cohort study is longitudinal and prospective, consisting of a baseline (TO) and 3 follow-up surveys, covering a time period of on average 1.6 years $(T 0-T 1, S D=0.2), 3.5$ years $(T 0-T 2, S D=0.3)$, and 8.4 years $(T 0-T 3$, range $=7.3-10.5 \mathrm{y}, \mathrm{SD}=0.7)$. Because the primary goal of the study was to examine the incidence and developmental risk factors for psychopathology, the younger group (14-15 y), presumed to have the highest incidence density, was sampled at twice the rate of persons aged 16-21 years, and the oldest group (22-24 y) was sampled at half this rate. For the same reason, subjects aged 14-17 years at baseline were examined at the 4 time points and subjects aged 18-24 years were assessed only 3 times (TO, T2, T3). The present study is based on the whole cohort assessed at TO, T2 and T3. Response rates were $84 \%(N=2548)$ for T2 and $74 \%$ $(\mathrm{N}=2210)$ for T3.

\section{Instruments}

Participants were assessed using the computerized version of the Munich-Composite International Diagnostic Interview (DIA-X/M-CIDI) [25], an updated version of the World Health Organization's CIDI version 1.2 [26]. The DIA-X/M-CIDI is a comprehensive, fully standardized diagnostic interview and assesses symptoms, syndromes, and diagnoses of various mental disorders in accordance with the definitions and criteria of the International Classification of Diseases, Tenth Revision, and Diagnostic and Statistical Manual of Mental Disorders (Fourth Edition), along 
with information about onset, duration, severity of symptoms, and psychosocial impairment. The CIDI has been primarily designed for use in epidemiological studies of mental disorders and can also be used for clinical purposes. It is divided into 16 sections: 1 sociodemographic section, 12 sections assessing 288 symptoms of groups of mental disorders (including 'somatoform and dissociative,' 'phobic and other anxiety,' 'depressive and dysthymic,' 'manic and bipolar affective,' 'schizophrenia and other psychotic,' 'eating,' 'dementia and other cognitive,' 'posttraumatic stress,' as well as 'tobacco,' 'alcohol,' and 'substance-related' disorders), and 3 final sections containing concluding questions, interviewer observations, and interviewer ratings. The instrument, designed for use by trained interviewers who are not clinicians, has shown high inter-rater [27, 28] and test-retest reliability [25, 29]. However, the assessment of psychosis with CIDI by lay interviewers is not considered reliable. Therefore, in the EDSP Study, trained and experienced clinical interviewers at the level of clinical psychologist, who were allowed to probe with follow-up clinical questions, conducted the interviews in the respondents' homes. At baseline, the DIA$\mathrm{X} / \mathrm{M}$-CIDI lifetime version was used. At each of the follow-up assessments, participants applied the interval version, which covers the period of assessment from the last interview until the next. Data on the $G$ section concerning psychosis and its clinical relevance were collected at T2 (lifetime version) and T3 (interval version).

\section{Assessment of psychotic experiences}

Data on positive psychotic experiences were collected at time T2 (lifetime version) and T3 (interval version) using the G-section of the DIA-X/M-CIDI. Presence of positive psychotic experiences was broadly defined as any rating of 'present' on any of the 20 DIAX/M-CIDI core psychosis items (G1, G2a, G3-G5, G7-G13, G13b, G14, G17, G18, G20, G20C, G21, and G22a), including 14 delusion items, 5 hallucination items, and 1 item on passivity phenomena. Items relate to classic psychotic symptoms involving, e.g., persecution, thought interference, auditory hallucinations, and passivity phenomena. Participants were first invited by the psychologist to read a list of all the psychotic experiences and then asked whether they ever experienced such symptoms. All these psychosis items were rated in 2 ways: 'absent' and 'present'. Thus, all the psychosis DIA-X/M-CIDI items used in the present study were coded in a dichotomous manner without intermediate levels.

\section{Assessment of urbanicity}

Urbanicity was assessed at baseline and was defined dichotomously as either living in the city of Munich (4061 persons per square mile) or in the rural surroundings (553 persons per square mile) at time of inclusion. Data on living location were derived from the population registry offices of the city and the 29 counties of Munich. 
Assessment of cannabis use

Cannabis use was assessed with the L-section of the DIA-X/M-CIDI at all three assessments. Conform previous analyses [11], cannabis use at TO was defined as lifetime use of cannabis of five times or more ('TO cannabis use') and cannabis use at T2 was defined as use of cannabis of five times or more since TO ('T2 cannabis use').

\section{Statistical analyses}

Data were analyzed using STATA, release 10.0 (StataCorp, College Station, TX). Associations are expressed as risk differences (RD) from logistic regression models.

\section{Cannabis $X$ urbanicity interaction}

To investigate whether the effects of incident cannabis use over the T0-T2 period on expression of incident psychotic experiences over the T2-T3 period were moderated by the urban environment, interaction between TO-T2 cannabis use and urbanicity was calculated under an additive model $[22,23]$. This was done as the additive interaction can be interpreted in a meaningful way, yielding information on the extent to which two causes biologically depend on each other or co-participate in disease causation [30]. Thus, we derived from the additive statistical interaction an estimate of the amount of biological synergism, as originally described by Darroch [30], and since successfully applied to psychiatric research [31-33]. The amount of biological synergism in this context can be understood as the proportion of individuals exposed to both cannabis use and urbanicity that developed the psychosis outcome because of the specific co-participation of these two factors. If this proportion is low, the statistical interaction is of little practical importance as most individuals exposed to both cannabis use and urbanicity will have developed the outcome because of either cannabis or urbanicity in isolation, not because of their synergistic action [30]. How the amount of biological synergism can be estimated approximately from the additive statistical interaction has been described in detail previously [30, 33]. To ensure prediction of strictly incident psychotic symptoms over the T2-T3 follow-up period, all individuals who had reported lifetime psychotic experiences at T2 were excluded from the analyses. The interaction between TO-T2 cannabis use and urbanicity was calculated using the BINREG procedure in STATA yielding RD's, followed by calculation of the appropriate linear combinations from the model with the interaction, using the STATA LINCOM command. In addition, in order to check for possible mediation between cannabis and urbanicity (in the sense of one factor influencing the occurrence of the other), logistic regression analysis was used to test whether urbanicity was associated with TO cannabis use.

All analyses were a priori adjusted for the following confounding risk factors: age (in years), sex $(0=$ female, $1=$ male), socio-economic status (lower, middle, upper, 
other), cannabis use at baseline (to control for possible mediating effects between urbanicity and cannabis use), use of other drugs and childhood trauma [34]. Use of 'other drugs' included psychostimulants, sedatives, opiates, cocaine, phencyclidine and psychedelic drugs and refers to use of 5 times or more of any of these drugs, as assessed with the L-section of the M-CIDI. Conform previous analyses in this sample [35], 'Childhood trauma' was defined as lifetime experience of any traumatic experience during childhood as assessed with the $\mathrm{N}$-section of the M-CIDI, dichotomously defined as 'present' versus 'absent'.

\section{Results}

\section{Sample}

A total of 2210 individuals completed the T3 assessment (response rate $74.0 \%$ ). Two-hundred-ninety participants had partial missing information on substance use and psychotic symptoms, resulting in a final risk set for analysis of 1923 individuals of which $926(48.2 \%)$ were men. Mean age was 18.3 years (SD $=3.3$ years) at TO, 21.8 (SD $=3.4$ years) at $\mathrm{T} 2$ and 26.6 (SD $=3.5$ years) at T3. Participant characteristics of the risk set are summarized in table 1 .

At T0, 247 participants (12.8\%) reported lifetime cannabis use. At T2, 392 participants $(20.4 \%)$ reported using cannabis over the TO-T2 interval and 381 individuals $(27.1 \%$ ) reported T2-T3 interval cannabis use at T3. Psychotic symptoms were reported by 436 participants $(22.7 \%$ - lifetime) at T2 and by 231 participants (12.0\% - interval) at T3. At the time of inclusion, $1.345(69.9 \%)$ participants were registered as living in the city of Munich; 578 participants $(30.1 \%)$ were registered as living in the rural surroundings.

\section{Main effects of cannabis and urbanicity}

In individuals with no history of psychotic symptoms, cannabis use over the TO-T2 period was significantly associated with incident psychotic symptoms over the T2-T3 period (unadjusted $\mathrm{OR}=1.77,95 \% \mathrm{Cl}: 1.16-2.70, \mathrm{p}=0.008$ ). After adjustment for age, sex, socio-economic status, cannabis use at baseline, childhood trauma and use of other drugs, the strength of the association was reduced somewhat (adjusted OR $=1.59,95 \% \mathrm{Cl}: 0.98-2.60, \mathrm{p}=0.061)$. There was no significant association between urbanicity and incident psychotic symptoms over the T2-T3 period (adjusted $\mathrm{OR}=1.16,95 \% \mathrm{Cl}: 0.77-1.76, \mathrm{p}=0.497)$.

\section{Interaction between cannabis and urbanicity}

Analysis revealed a significant interaction between TO-T2 cannabis use and urbanicity (test for additive interaction: $10.9 \%$ adjusted difference in risk, $95 \% \mathrm{Cl}$ : $3.2-18.6, p=0.005$, table 2). The association between TO-T2 cannabis use and T2- 
T3 psychosis was much stronger for individuals from an urban environment (adjusted RD 6.8\%, 95\% Cl: $1-12.5, \mathrm{p}=0.021$, table 2) compared to individuals from rural surroundings (adjusted RD $-4.1 \%, 95 \% \mathrm{Cl}:-9.8-1.6, \mathrm{p}=0.159$, table 2).

Further analyses revealed that biological synergism was between $51 \%$ and $66 \%$, i.e. of all the individuals in the risk set exposed to both urbanicity and cannabis, the majority had developed the psychosis outcome because of the specific synergistic effect of the two, assuming causality. There was a small but significant positive association between urbanicity and TO cannabis use (adjusted OR $=1.40,95 \% \mathrm{Cl}$ : $1.02-1.98, p=0.038)$.

Table 1. Participant characteristics of the risk set $(N=1923)$.

\begin{tabular}{|c|c|c|c|}
\hline \multirow{2}{*}{\multicolumn{2}{|c|}{ Gender }} & $n$ & $\%$ \\
\hline & & & \\
\hline & Male & 926 & 48.2 \\
\hline & Female & 997 & 51.8 \\
\hline \multicolumn{4}{|c|}{ Socio-economic status } \\
\hline & Lower & 115 & 6 \\
\hline & Middle & 1114 & 58 \\
\hline & Upper & 661 & 34.4 \\
\hline & Other & 33 & 1.7 \\
\hline \multicolumn{4}{|c|}{ Urbanicity } \\
\hline & Urban & 1345 & 69.9 \\
\hline & Rural & 578 & 30.1 \\
\hline \multicolumn{4}{|c|}{ Childhood trauma } \\
\hline & Yes & 363 & 18.9 \\
\hline & No & 1560 & 81.1 \\
\hline \multicolumn{4}{|c|}{ Cannabis use (TO) } \\
\hline & Yes & 247 & 12.8 \\
\hline & No & 1676 & 87.2 \\
\hline \multicolumn{4}{|c|}{ Use of other drugs (TO) } \\
\hline & Yes & 36 & 1.9 \\
\hline & No & 1887 & 89.1 \\
\hline
\end{tabular}

Note. Socio-economic status: lower (lower class, lower middle class), middle (middle middle class), upper (higher middle class, upper class) other (none of the above or missing). Urbanicity: urban (the city of Munich, 4061 persons per square mile), rural (the surroundings of Munich, 553 persons per square mile). Cannabis use/Use of other drugs: Any use of more than five times. Childhood trauma: Any traumatic experience during childhood. 
\begin{tabular}{l|l} 
CHAPTER 3 & 55
\end{tabular}

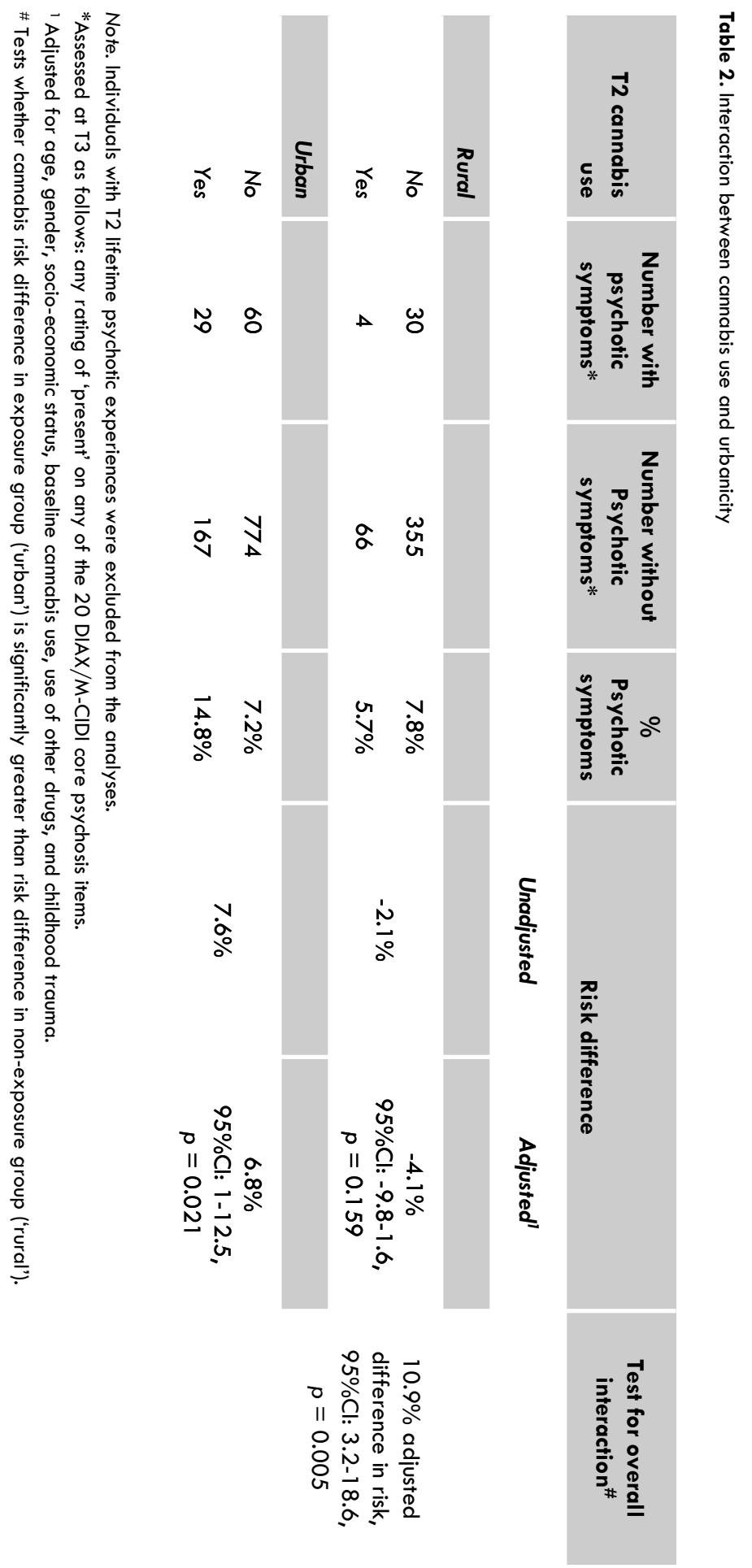




\section{Discussion}

There was evidence that urbanicity may moderate the long-term effects of cannabis on psychosis: adolescents who grew up in the city of Munich were much more likely to develop psychotic symptoms after cannabis use than individuals who grew up in the rural surroundings of Munich. This interaction effect was independent of confounding factors such as age, gender, socio-economic status, use of other drugs and childhood trauma and was not irrelevant, as the majority of those exposed to both urbanicity and cannabis developed the psychosis outcome because of their co-action. There was also some evidence that this interaction could be interpreted in the sense of mediation (urbanicity leading to cannabis use leading to psychosis), as a small but significant association existed between urbanicity and cannabis use.

\section{Cannabis and urbanicity: moderation and possible mechanisms}

The present study identified urbanicity as one of the factors that may moderate the association between cannabis and psychosis. Previously, a similar interaction was reported between cannabis and developmental trauma, another environmental exposure associated with psychotic outcomes [15, 16]. Given evidence that cannabis, trauma, and urbanicity do not reflect the same environmental influence in the association with psychotic outcomes [4], the current analyses, in combination with the findings on similar moderation by developmental trauma $[15,16]$, suggest that they do share the same interactive pathway. A mechanism of developmental sensitization has been suggested to underlie this pathway [17].

Epidemiological evidence indicates that although developmental expression of subclinical psychotic experiences in adolescence is mostly transient, repeated exposure to environmental risk factors causes subclinical psychotic experiences to persist and become more severe, resulting in onset of psychotic illness in a minority of individuals $[4,36,37]$. Urbanicity represents a complex proxy environmental influence, and the mechanism of its impact on psychosis remains largely unknown. The effect does not appear to be mediated by demographic factors, obstetric complications, childhood socio-economic position, neuropsychological impairment, air pollution, drug use or ethnic group, and there is evidence that exposure between the ages of 5-15 years is associated with the greatest effect $[20,21,38]$, suggesting mediation by factors impacting during development. One study showed that an urban environment induced anxiety, negative feelings towards other people and reasoning biases associated with delusional ideation [39]. Allardyce and colleagues [40] showed that social fragmentation as well as deprivation had a significant effect on first psychosis admission rates independently of urbanicity, and that the effect of urbanicity on first psychosis admission rates disappeared after statistical adjustment for social fragmentation and deprivation [40]. Similarly, there is evidence that risk 
for psychotic syndrome associated with indicators of social maladjustment, for example single parent family, single marital status and residential instability, similarly varies with the degree to which this represents the exception in relation to the wider social environment [41, 42]. This type of interaction between individuallevel and area-level social "fragmentation" may mediate the effect of the urban environment [41].

The absence of an association between urban environment and incident psychotic symptoms in the current study is in line with previous research [43]. The likely explanation is that the time window of exposure to urban environment in relation to risk for psychosis outcomes is from 5-15 years [44]. Individuals with lifetime psychotic symptoms, assessed at baseline, that previous work in this sample showed were associated with urban environment [45], were excluded from the analyses. Later onset 'incident' psychotic symptoms could not be traced directly anymore to earlier exposure to the urban environment, although an indirect effect was still apparent, through moderation of sensitivity to the psychotomimetic effects of cannabis. It may be hypothesized that early exposure, i.e. between the ages of 5-15 years, to increased social fragmentation associated with an urban environment may constitute an environmental stressor that, through epigenetic mechanisms, leads to permanent neurodevelopmental alterations, which in turn result may contribute to enhanced sensitivity to the psychotomimetic effects of cannabis later in life.

\section{Cannabis and urbanicity: Moderation or mediation?}

In the present study, a small but significant association was found between urbanicity and cannabis use: Individuals living in the urban environment were more likely to use cannabis at baseline than individuals from the rural area. This finding indicates that the interaction between cannabis use and urbanicity may represent not only an underlying mechanism of moderation (the psychotomimetic effect of cannabis is larger in urban areas) but also of mediation (living in the city may enhance cannabis use). Both mechanisms would be important form a public health perspective.

\section{Cannabis $X$ urbanicity: What it does and what it does not tell us?}

One of the limitations of epidemiological research concerns the inability to make inferences about underlying molecular mechanisms. Therefore, our findings are not informative with regard to molecular mechanisms such as developmental sensitization. However, when epidemiological research is followed by targeted experimental animal and human research, it may constitute the first step in a chain of research efforts to uncover the biological mechanisms of environment-environment or gene-environment interaction [7]. Thus, the fact that the majority of those exposed to both cannabis and urbanicity developed the psychosis outcome because of a specific mechanism of co-participation between these two factors suggests the finding 
constitutes an important starting point requiring follow-up experimental research targeting a final common pathway that both factors may impact on.

The interest of epidemiological findings like these lies in the area of public health and disease causation. As shown by Darroch [30], evidence of non-additivity represents a way to estimate the population amounts of biological synergism (the population exposed to both risk factors that developed the outcome because of the specific co-participation between the two factors) and biological parallelism (the population exposed to both risk factors that developed the outcome because of the action of only one factor). Examination of biological synergism and parallelism is of vital interest to epidemiologists and public health, since it allows identification of populations that would benefit most from a given intervention: If synergism is large, public health gains could be obtained by targeting either factor. On the other hand, if parallelism is large, public health may gain by targeting both factors. In the current study, synergism was between 50-65\%, indicating that targeting either factor could potentially result in public health gains.

\section{Limitations/methodological issues}

There are several limitations. First, information on substance use and psychosis outcome was acquired using the M-CIDI, which is meant to essentially provide selfreported information. Nevertheless, the interview was conducted face-to-face by clinical psychologists who were allowed to follow up with clinical questioning in order to ensure systematic and valid assessment of outcomes and can therefore be assumed to yield better and more valid results than a self-report questionnaire.

Secondly, urbanicity was assessed by retrieving data on living location from the German registry offices. Although these registers can be considered as highly accurate since in Germany registration is obligatory and registries are regularly updated, the information is restricted to current living location and therefore does not provide information on duration of living at a certain location. Pedersen en Mortensen [44] showed that increasing duration of living in urban areas leads to increased psychosis risk in a dose-response fashion. Research has furthermore suggested that the effect of urbanicity on psychosis risk operates during upbringing rather than at birth [44] or around illness onset [46]. Considering the current study, even under the hypothetical assumption that participants registered as living in the city of Munich had just moved there from the rural surroundings or, the other way around, individuals from the rural surroundings had just moved to the city, this would have yielded an under-, rather than an overestimation of the effects size. In addition, previous work suggest current urban residence in young people reflects urban upbringing in the majority [46]. The paradigm used in the current study was based on the notion that psychotic symptoms can be expressed below the threshold 
of diagnosis and are meaningful in relation to the clinical phenotype. Meta-analytic work of sub-diagnostic expression of psychotic experiences in the general population has shown aetiological, psychopathological and longitudinal continuity between the extended subclinical phenotype and clinical expression of psychotic disorder, as well as a similar age-related developmental pattern of expression [47]; a recent landmark general population birth cohort study confirmed this pattern of findings [48]. Further, since transient and normally expressed psychotic experiences may, under certain circumstances, become abnormally persistent and develop into clinical psychosis [4, 36, 49], psychotic experiences represent an important phenotype for the investigation of mechanisms and pathways by which environmental risk factors such as cannabis impact on psychosis risk [5].

Finally, in interpreting the findings of this current study, it was assumed that in this area in Germany, cannabis used in the city does not differ from cannabis used in rural areas. Although this cannot be formally explored the assumption may be valid, since availability of cannabis in Germany is limited in general (compared to countries such as the Netherlands, where 'coffeeshops' are allowed to sell cannabis on a semi-legal basis) and access to cannabis is likely comparable between rural and urban areas. 


\section{References}

1. Henquet, C., et al., The environment and schizophrenia: the role of cannabis use. Schizophrenia Bulletin, 2005. 31(3): p. 608-12.

2. Moore, T.H., et al., Cannabis use and risk of psychotic or affective mental health outcomes: a systematic review. Lancet, 2007. 370(9584): p. 319-28.

3. Semple, D.M., A.M. Mclntosh, and S.M. Lawrie, Cannabis as a risk factor for psychosis: systematic review. Journal of Psychopharmacology, 2005. 19(2): p. 187-94.

4. Cougnard, A., et al., Does normal developmental expression of psychosis combine with environmental risk to cause persistence of psychosis? A psychosis proneness-persistence model. Psychological Medicine, 2007. 37(4): p. 513-27.

5. Kuepper, R., et al., Continued cannabis use and risk of incidence and persistence of psychotic symptoms: 10 year follow-up cohort study. British Medical Journal, 2011 . 342: p. d738.

6. Henquet, C., et al., Gene-environment interplay between cannabis and psychosis. Schizophrenia Bulletin, 2008. 34(6): p. $1111-21$.

7. van Os, J., G. Kenis, and B.P. Rutten, The environment and schizophrenia. Nature, 2010. 468(7321): p. 203-12.

8. Caspi, A., et al., Moderation of the effect of adolescent-onset cannabis use on adult psychosis by a functional polymorphism in the catechol-O-methyltransferase gene: longitudinal evidence of a gene $X$ environment interaction. Biological Psychiatry, 2005. 57(10): p. 1117-27.

9. Lieb, R., et al., The Early Developmental Stages of Psychopathology Study (EDSP): a methodological update. European Addiction Research, 2000. 6(4): p. 170-82.

10. Wittchen, H.U., et al., Early developmental stages of psychopathology study (EDSP): objectives and design. European Addiction Research, 1998. 4(1-2): p. 18-27.

11. Henquet, C., et al., Prospective cohort study of cannabis use, predisposition for psychosis, and psychotic symptoms in young people. British Medical Journal, 2005. 330(7481): p. 11.

12. G.R.O.U.P., Evidence that familial liability for psychosis is expressed as differential sensitivity to cannabis: an analysis of patient-sibling and sibling-control pairs. Archives of General Psychiatry, $2011.68(2):$ p. 138-47.

13. Henquet, C., et al., An experimental study of catechol-o-methyltransferase Val158Met moderation of delta-9-tetrahydrocannabinol-induced effects on psychosis and cognition. Neuropsychopharmacology, 2006. 31(12): p. 2748-57.

14. van Winkel, R., Family-Based Analysis of Genetic Variation Underlying Psychosis-Inducing Effects of Cannabis: Sibling Analysis and Proband Follow-up. Archives of General Psychiatry, 201 1. 68(2): p. 148-57.

15. Houston, J.E., et al., Childhood sexual abuse, early cannabis use, and psychosis: testing an interaction model based on the National Comorbidity Survey. Schizophrenia Bulletin, 2008. 34(3): p. 580-5.

16. Harley, M., et al., Cannabis use and childhood trauma interact additively to increase the risk of psychotic symptoms in adolescence. Psychological Medicine, 2009: p. 1-8.

17. Collip, D., I. Myin-Germeys, and J. Van Os, Does the concept of "sensitization" provide a plausible mechanism for the putative link between the environment and schizophrenia? Schizophrenia Bulletin, 2008. 34(2): p. 220-5.

18. Lardinois, M., et al., Childhood trauma and increased stress sensitivity in psychosis. Acta Psychiatrica Scandinavica, 2011 . 123(1): p. 28-35.

19. van Winkel, R., N.C. Stefanis, and I. Myin-Germeys, Psychosocial stress and psychosis. A review of the neurobiological mechanisms and the evidence for gene-stress interaction. Schizophrenia Bulletin, 2008. 34(6): p. 1095-105.

20. Kelly, B.D., et al., Schizophrenia and the city: A review of literature and prospective study of psychosis and urbanicity in Ireland. Schizophrenia Research, 2010. 116(1): p. 75-89. 
21. Krabbendam, L. and J. van Os, Schizophrenia and urbanicity: a major environmental influence-conditional on genetic risk. Schizophrenia Bulletin, 2005. 31 (4): p. 795-9.

22. van Os, J., C.B. Pedersen, and P.B. Mortensen, Confirmation of synergy between urbanicity and familial liability in the causation of psychosis. American Journal of Psychiatry, 2004. 161(12): p. 2312-4.

23. van Os, J., et al., Do urbanicity and familial liability coparticipate in causing psychosis? American Journal of Psychiatry, 2003. 160(3): p. 477-82.

24. Weiser, M., et al., Social and cognitive functioning, urbanicity and risk for schizophrenia. British Journal of Psychiatry, 2007. 191: p. 320-4.

25. Wittchen, H.U., et al., Test-retest reliability of the computerized DSM-IV version of the MunichComposite International Diagnostic Interview (M-CIDI). Social Psychiatry and Psychiatric Epidemiology, 1998. 33(1 1): p. 568-78.

26. Robins, L.N., et al., The Composite International Diagnostic Interview. An epidemiologic Instrument suitable for use in conjunction with different diagnostic systems and in different cultures. Archives of General Psychiatry, 1988. 45(12): p. 1069-77.

27. Wittchen, H.U., et al., Cross-cultural feasibility, reliability and sources of variance of the Composite International Diagnostic Interview (CIDI). The Multicentre WHO/ADAMHA Field Trials. British Journal of Psychiatry, 1991. 159: p. 645-53, 658.

28. Cottler, L.B., et al., Agreement between DSM-III and III-R substance use disorders. Drug and Alcohol Dependence, 1991. 29(1): p. 17-25.

29. Wittchen, H.U., Reliability and validity studies of the WHO--Composite International Diagnostic Interview (CIDI): a critical review. Journal of Psychiatry Research, 1994. 28(1): p. 57-84.

30. Darroch, J., Biologic synergism and parallelism. American Journal of Epidemiology, 1997. 145(7): p. $661-8$.

31. Corcoran, C. and D. Malaspina, Traumatic brain injury and schizophrenia. International Journal of Mental Health, 2001. 30: p. 17-33.

32. Clarke, M.C., et al., Evidence for an interaction between familial liability and prenatal exposure to infection in the causation of schizophrenia. American Journal of Psychiatry, 2009. 166(9): p. 1025 30 .

33. van Os, J., et al., Cannabis use and psychosis: a longitudinal population-based study. American Journal of Epidemiology, 2002. 156(4): p. 319-27.

34. Read, J., et al., Childhood trauma, psychosis and schizophrenia: a literature review with theoretical and clinical implications. Acta Psychiatrica Scandinavica, 2005. 112(5): p. 330-50.

35. Spauwen, J., et al., Evidence that the outcome of developmental expression of psychosis is worse for adolescents growing up in an urban environment. Psychological Medicine, 2006. 36(3): p. 407-15.

36. Dominguez, M.D., et al., Evidence that onset of clinical psychosis is an outcome of progressively more persistent subclinical psychotic experiences: an 8-year cohort study. Schizophrenia Bulletin, 2011. 37(1): p. 84-93.

37. Mackie, C.J., N. Castellanos-Ryan, and P.J. Conrod, Developmental trajectories of psychotic-like experiences across adolescence: impact of victimization and substance use. Psychological Medicine, $2011.41(1)$ : p. $47-58$.

38. March, D., et al., Psychosis and place. Epidemiologic Reviews, 2008. 30: p. 84-100.

39. Ellett, L., D. Freeman, and P.A. Garety, The psychological effect of an urban environment on individuals with persecutory delusions: the Camberwell walk study. Schizophrenia Research, 2008. 99(1-3): p. 77-84.

40. Allardyce, J., et al., Social fragmentation, deprivation and urbanicity: relation to first-admission rates for psychoses. British Journal of Psychiatry, 2005. 187: p. 401-6.

41. Zammit, S., et al., Individuals, schools, and neighborhood: a multilevel longitudinal study of variation in incidence of psychotic disorders. Archives of General Psychiatry, 2010. 67(9): p. 914-22. 
42. van Os, J., et al., Neighbourhood variation in incidence of schizophrenia. Evidence for personenvironment interaction. British Journal of Psychiatry, 2000. 176: p. 243-8.

43. Wiles, N.J., et al., Self-reported psychotic symptoms in the general population: results from the longitudinal study of the British National Psychiatric Morbidity Survey. British Journal of Psychiatry, 2006. 188: p. 519-26.

44. Pedersen, C.B. and P.B. Mortensen, Evidence of a dose-response relationship between urbanicity during upbringing and schizophrenia risk. Archives of General Psychiatry, 2001. 58(11): p. 1039-46.

45. Spauwen, J., et al., Does urbanicity shift the population expression of psychosis? Journal of Psychiatric Research, 2004. 38(6): p. 613-8.

46. Marcelis, M., N. Takei, and J. van Os, Urbanization and risk for schizophrenia: does the effect operate before or around the time of illness onset? Psychological Medicine, 1999. 29(5): p. 1197-203.

47. van Os, J., et al., A systematic review and meta-analysis of the psychosis continuum: evidence for a psychosis proneness-persistence-impairment model of psychotic disorder. Psychological Medicine, 2009. 39(2): p. 179-95.

48. Polanczyk, G., et al., Etiological and clinical features of childhood psychotic symptoms: results from a birth cohort. Archives of General Psychiatry, 2010. 67(4): p. 328-38.

49. Hanssen, M., et al., The incidence and outcome of subclinical psychotic experiences in the general population. British Journal of Clinical Psychology, 2005. 44(P+ 2): p. 181-91. 
\begin{tabular}{l|l} 
CHAPTER 3 & 63
\end{tabular} 



\title{
NON-REPLICATION OF INTERACTION BETWEEN CANNABIS USE AND TRAUMA IN PREDICTING
} PSYCHOSIS

\author{
Rebecca Kuepper ${ }^{1}$, Cécile Henquet ${ }^{1}$ 3, Roselind Lieb ${ }^{4}$, Hans-Ulrich \\ Wittchen ${ }^{5} \&$ Jim van $\mathrm{Os}^{1,2}$
}

\footnotetext{
1 Department of Psychiatry and Neuropsychology, South Limburg Mental Health Research and Teaching Network, EURON, Maastricht University Medical Center, PO Box 616 (location Vijverdal), NL-6200 MD, Maastricht, The Netherlands

2 King's College London, King's Health Partners, Department of Psychosis Studies, Institute of Psychiatry, London, United Kingdom 3 PsyQ Heerlen, Mondriaan, South Limburg, The Netherlands ${ }^{4}$ Institute of Psychology, University of Basel, Birmannsgasse 8, CH-4009 Basel, Switzerland 5 Institute of Clinical Psychology and Psychotherapy, Technical University Dresden, Chemnitzerstrasse 46, D-01 187 Dresden, Germany
}

Schizophrenia Research, 2011 . 131(1-3): p. 262-263. 
NON-REPLICATION OF INTERACTION BETWEEN CANNABIS USE AND TRAUMA IN PREDICTING PSYCHOSIS 


\section{Dear Editors,}

Cannabis use is considered a component cause of psychotic disorder interacting with genetic and environmental risk factors in increasing psychosis risk [1]. Recently, two cross-sectional and one prospective study provided evidence that cannabis use interacts additively with trauma to increase psychosis risk [2-4]. In an attempt at further replication, we examined prospective data from the German Early Developmental Stages of Psychopathology (EDSP) study $[5,6]$.

The EDSP study collected data on the prevalence, incidence, risk factors, comorbidity, and course of mental disorders in a random, representative population sample of adolescents and young adults in the general population [5, 6]. Individuals were assessed three times (at TO, T2, and T3) over a 10-year follow-up period. More details on the sampling, representativeness, instruments, procedures, and statistical methods of the EDSP Study sample have been presented elsewhere $[5,6]$. Data on psychotic symptoms, cannabis use and trauma were acquired with the computerized version of the Munich-Composite International Diagnostic Interview (DIA-X/M-CIDI) [7], an updated version of the World Health Organization's CIDI version 1.2 [8]. At TO, the DIA-X/M-CIDI lifetime version was used. At each of the follow-up assessments, participants applied the interval version, covering the period of assessment from the last interview until the next. Data on positive psychotic symptoms were collected at T2 (lifetime version, representing lifetime experience of symptoms) and T3 (interval version, representing symptoms that occurred over the T2-T3 period). Presence of positive psychotic experiences was broadly defined as any rating of 'present' on any of the DIAX/M-CIDI core psychosis items. All items were dichotomously rated as 'absent' or 'present'. Cannabis use was assessed at all three assessments. Conform previous analyses [9], cannabis use at TO was defined as lifetime use of cannabis of five times or more and cannabis use at T2 was defined as use of cannabis of five times or more since TO. Trauma was assessed at TO and was dichotomously defined as having experienced any of the following events at least once lifetime: war experiences, physical threats or attacks, rape, sexual abuse, natural disasters, serious accidents, kidnapping and hostage-taking, and witnessing any of the aforementioned events.

Data were analyzed using STATA, release 11.1 (StataCorp, College Station, TX). Associations were expressed as odds ratios (OR) derived from logistic regression models. Interaction between T2 cannabis use and trauma was calculated under an additive model [10], using the BINREG procedure in STATA yielding risk differences $(R D)$, followed by calculation of the appropriate linear combinations from the model with the interaction, using the STATA LINCOM command. To ensure prediction of strictly incident psychotic symptoms over the T2-T3 follow-up period, all individuals 
who had reported lifetime psychotic experiences at T2 were excluded from the analyses. Analyses were adjusted for the following confounding risk factors: age (in years), sex ( $0=$ female, $1=$ male), socio-economic status (lower, middle, upper, other), cannabis use at baseline, use of other drugs at baseline

(including psychostimulants, sedatives, opiates, cocaine, phencyclidine and psychedelic drugs), and urbanicity, defined dichotomously as living in the city of Munich ('urban', 4061 persons per square mile) or in the rural surroundings ('rural', 553 persons per square mile) at the time of inclusion.

We analyzed data of 1923 individuals of which $926(48.2 \%)$ were men. Mean age was 18.3 years (SD $=3.3$ years) at T0, 21.8 (SD = 3.4 years) at T2 and 26.6 (SD $=3.5$ years) at T3. There was no evidence that trauma moderated the association between T2 cannabis use and incident psychotic symptoms over the T2-T3 period (see table 1 for statistics). The interaction remained small and non-significant when examining a more stringent outcome criterion, defined as having experienced at least two psychotic symptoms, and when examining the influence of having been exposed to at least two or three traumatic events, respectively. In order to strictly predict incident psychotic symptoms over the T2-T3 period, the above described analyses excluded all individuals with lifetime pre-existing psychotic symptoms as assessed at T2. Accordingly, the remaining group of subjects exclusively consisted of individuals who had not developed any psychotic symptoms by the time of the T2 assessment, possibly constituting a relatively resilient subgroup. However, the interaction remained non-significant also when analyzing the whole cohort $(-1.3 \%$ adjusted difference in risk, $95 \% \mathrm{Cl}:-11.1-8.4, \mathrm{p}=0.782$ ).

Opposed to what was hypothesized and in contrast to previous findings [2-4], the current analyses did not provide evidence for interaction between cannabis use and trauma in increasing psychosis risk. This may be due to sampling variation, or alternatively, the relatively long follow-up between T2 and T3 was insensitive to this type of analysis. More work in the area of environment-environment interactions in predicting psychosis is necessary. 

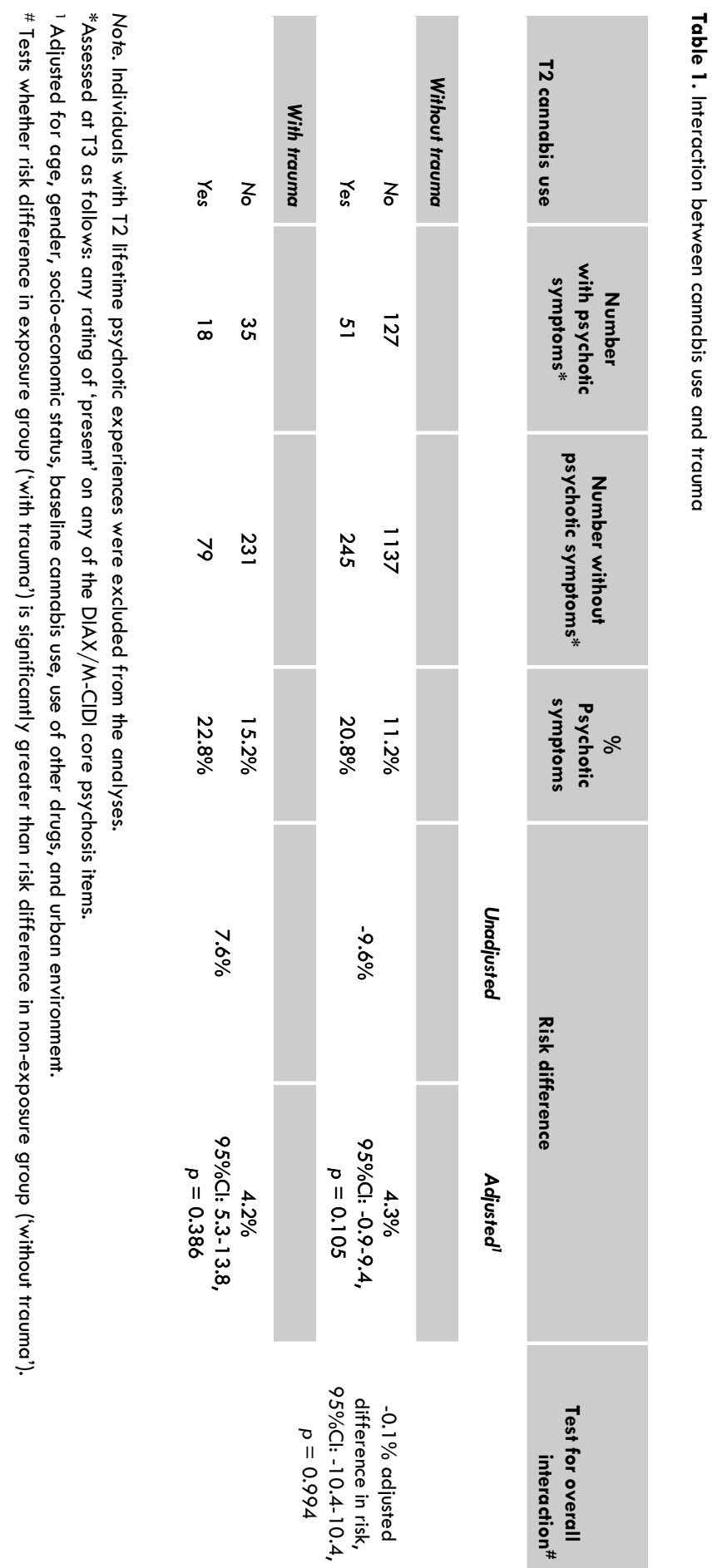

$\frac{1}{2}$
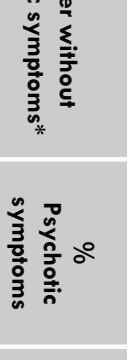

 


\section{References}

1. Henquet, C., et al., Gene-environment interplay between cannabis and psychosis. Schizophrenia Bulletin, 2008. 34(6): p. $1111-21$.

2. Houston, J.E., et al., Childhood sexual abuse, early cannabis use, and psychosis: testing an interaction model based on the National Comorbidity Survey. Schizophrenia Bulletin, 2008. 34(3): p. 580-5.

3. Harley, M., et al., Cannabis use and childhood trauma interact additively to increase the risk of psychotic symptoms in adolescence. Psychological Medicine, 2010. 40(10): p. 1627-34.

4. Konings, M., et al., Replication in two independent population-based samples that childhood trauma interacts with cannabis use on psychosis risk. Psychological Medicine, in press.

5. Wittchen, H.U., et al., Early developmental stages of psychopathology study (EDSP): objectives and design. European Addiction Research, 1998. 4(1-2): p. 18-27.

6. Lieb, R., et al., The Early Developmental Stages of Psychopathology Study (EDSP): a methodological update. European Addiction Research, 2000. 6(4): p. 170-82.

7. Wittchen, H.U., et al., Test-retest reliability of the computerized DSM-IV version of the MunichComposite International Diagnostic Interview (M-CIDI). Social Psychiatry and Psychiatric Epidemiology, 1998. 33(1 1): p. 568-78.

8. Robins, L.N., et al., The Composite International Diagnostic Interview. An epidemiologic Instrument suitable for use in conjunction with different diagnostic systems and in different cultures. Archives of General Psychiatry, 1988. 45(12): p. 1069-77.

9. Kuepper, R., et al., Continued cannabis use and risk of incidence and persistence of psychotic symptoms: 10 year follow-up cohort study. British Medical Journal, 2011 . 342: p. d738.

10. Darroch, J., Biologic synergism and parallelism. American Journal of Epidemiology, 1997. 145(7): $p$. 661-8. 



\title{
THE DOPAMINE DYSFUNCTION IN PSYCHOSIS REVISITED: NEW INSIGHTS INTO TOPOGRAPHY AND COURSE
}

\author{
Rebecca Kuepper ${ }^{1}$, Mette Skinbjerg ${ }^{2}$ \& Anissa Abi-Dargham ${ }^{2}$
}

\footnotetext{
1 Department of Psychiatry and Neuropsychology, South Limburg Mental Health Research and Teaching Network, EURON, Maastricht University Medical Center, PO Box 616 (location Vijverdal), NL-6200 MD, Maastricht, The Netherlands 2 Department of Psychiatry, Columbia College of Physicians and Surgeons, New York State Psychiatric Institute, Columbia University, New York, NY, USA
}

$$
\text { In press for }
$$

Handbook of Experimental Pharmacology: Current antipsychotics. 


\begin{abstract}
Schizophrenia has long been associated with an imbalance in dopamine (DA) neurotransmission, and brain imaging has played an important role in advancing our knowledge and providing evidence for the dopaminergic abnormalities. This chapter reviews the evidence for DA dysfunction in different brain regions in schizophrenia, in particular striatal, extrastriatal and prefrontal regions, with emphasis on recently published findings. As opposed to the traditional view that most striatal dopaminergic excess, associated with the positive symptoms of schizophrenia, involves the dopaminergic mesolimbic pathway, recent evidence points to the nigrostriatal pathway as the area of highest dysregulation. Furthermore, evidence from translational research suggests that dopaminergic excess may be present in the prodromal phase, and may by itself, as suggested by the phenotype observed in transgenic mouse with developmental overexpression of dorso-striatal D2 receptors, be an early pathogenic condition, leading to irreversible cortical dysfunction.
\end{abstract}




\section{Introduction}

The psychotomimetic effects of stimulant drugs, in combination with the observed antipsychotic effects of D2-blocking drugs, gave rise to the initial dopamine (DA) hypothesis of schizophrenia [1-3]. Refined and modified in the intervening years, this theory to date remains central to the pathophysiology of schizophrenia [4-6].

Based on the observation that DA-enhancing drugs such as amphetamine show psychotomimetic properties while the effectiveness of classic antipsychotic medication, blocking DA D2 receptors, had been shown to directly correlate with its affinity for the D2 receptor [7], the dopamine hypothesis initially assumed a general dopaminergic hyperfunction [1, 2]. However, subsequent findings, including reduced cerebral blood flow in the prefrontal cortex (PFC) of patients with schizophrenia and the observation that negative symptoms such as flattened affect, anhedonia and compromised cognitive function did not respond to classic antipsychotic treatment targeting D2 receptors, were incompatible with a generally overactive DA system. Accordingly, the dopamine hypothesis was reformulated as an imbalance in DA neurotransmission in different regions of the brain, characterized in particular by hyperactivity in the subcortical DA pathway and hypoactivity in the cortical DA system [8-10]. It was also suggested that DA hyperactivity in subcortical brain regions, in particular striatal areas, accounted primarily for the positive symptoms of schizophrenia, while the negative symptoms were mostly associated with DA hypoactivity in cortical areas, particularly PFC [11].

In this chapter, we present the history as well as the latest research findings relating to dopamine dysfunction in schizophrenia. The two main new findings that will be emphasized here are the following: Unlike the traditional thinking that most dopaminergic excess causing positive symptoms involves the dopaminergic mesolimbic pathway, recent evidence points to the nigrostriatal pathway, projecting to the anterior caudate, part of the associative striatum, as the area of highest dysregulation. Furthermore, as opposed to the thinking that dopamine is a final common pathway, recent evidence from translational research suggests that dopamine dysregulation may be an early pathogenic mechanism that leads to further dysregulation of brain function. We will review the evidence and outline future research needed to understand the molecular mechanisms and develop better therapeutic interventions. 


\section{Evidence for DA dysfunction in schizophrenia}

Direct empirical evidence for DA alterations in schizophrenia was initially elusive. Postmortem studies were difficult to interpret due to the possible confounding by prior neuroleptic exposure and the first imaging studies using Positron Emission Tomography (PET) and Single Photon Emission Tomography (SPECT) produced inconsistent findings. Advances in PET and SPECT imaging techniques, such as the development of new, high affinity radiotracers, made it possible to study DA neurotransmission in several regions of the brain, including striatal, extrastriatal and prefrontal cortical regions with more anatomical detail than previously possible.

\section{Striatal DA alterations}

Using PET and SPECT imaging techniques, different paradigms have been employed in order to study aspects of presynaptic and postsynaptic striatal DA alterations in schizophrenia. These include markers of DA synthesis, release and reuptake, as well as DA receptor availability and differences in receptor affinity states [12-14].

\section{Presynaptic DAergic parameters}

Dopamine synthesis. DA is synthesized by hydroxylation of the precursor tyrosine to L-DOPA, which then is decarboxylated to DA by aromatic acid decarboxylase (AADC). Radioactive analogues of L-DOPA, such as [ $\left.{ }^{1} \mathrm{C}\right] \mathrm{L}-\mathrm{DOPA}$ and [18F]DOPA, have been used to estimate enzymatic activity of $A A D C$, as an indicator of DA synthesis capacity [15-18]. However, this is overly simplified and other factors may affect the overall signal measured with $\left[{ }^{11} \mathrm{C}\right] \mathrm{L}-\mathrm{DOPA}$ and $\left[{ }^{18} \mathrm{~F}\right] \mathrm{DOPA}$, including delivery of $\left[{ }^{11} \mathrm{C}\right] \mathrm{L}-\mathrm{DOPA}$ and $\left[{ }^{18} \mathrm{~F}\right] \mathrm{DOPA}$ to the brain, crossing the plasma membrane, storage in presynaptic vesicles as a result of VMAT activity, release of $\left[{ }^{11} \mathrm{C}\right] \mathrm{L}-\mathrm{DOPA}$ and [ $\left.{ }^{18} \mathrm{~F}\right] \mathrm{DOPA}$, and degradation, all may play a role in addition to the activity of DOPA decarboxylase. Better knowledge of these mechanisms is needed to better understand the exact molecular processes underlying [ $\left.{ }^{1} \mathrm{C}\right] \mathrm{L}-\mathrm{DOPA}$ and [ ${ }^{18}$ F]DOPA uptake and their disturbance in schizophrenia. Nevertheless, a number of studies have used radiolabelled L-DOPA, such as [ ${ }^{1}$ C]L-DOPA or [18F]DOPA in schizophrenia. The first study, by Reith and colleagues [19], revealed significantly elevated [ ${ }^{8}$ F]DOPA uptake in drug-naivve patients compared to healthy controls [19]. While the majority of the following studies using the same technique replicated this finding [20-26], two studies failed to find markers of elevated DA synthesis capacity: One study reported a small but not significant increase in [ ${ }^{18}$ F]DOPA uptake [27], while another found decreased levels of [ ${ }^{8}$ F]DOPA uptake [28]. This discrepancy might be explained by differences in study population: Studies reporting positive findings were all conducted in acutely psychotic, mostly drug-naive, patients (with the exception of McGowan and colleagues), while the two 
studies reporting negative or inverse findings were done in chronic, previously medicated, patients. Recently, DA synthesis capacity was investigated in twins discordant for schizophrenia. No elevation in striatal DA synthesis was observed in the unaffected twin, nor in the low-symptomatic medicated twin with chronic schizophrenia when compared to controls, suggesting that increased DA synthesis is not a genetic marker for schizophrenia [29].

Dopamine release. The next step in DA transmission is the release of DA from the presynaptic terminal. An estimation of DA release from striatal DA neuron terminals can be obtained by measuring changes in D2 binding potential after pharmacologically induced DA release with SPECT (Single Emission Computed Tomography) and PET imaging techniques [30-32]. One of the first studies to investigate amphetamine-induced DA release in schizophrenia was conducted by Laruelle and colleagues [33], using SPECT and the radiotracer [123|]IBZM, an antagonist at $D 2 / 3$ receptors. Changes in $D 2 / 3$ binding potential as an estimation of amphetamine-induced DA release were measured in 15 medication-free patients with schizophrenia and 15 healthy control participants. The decrease in binding potential induced by amphetamine was significantly greater in patients with schizophrenia compared to controls. Furthermore, the amphetamine-induced decrease in D2/3 binding potential was associated with the transient increase in positive symptoms induced by amphetamine in the schizophrenia group [33]. The results of a subsequent PET study using [ $\left.{ }^{11} \mathrm{C}\right]$ raclopride as radiotracer were in line with these observations [34]: The schizophrenia group showed greater amphetamine-induced decreases in $\left[{ }^{1} \mathrm{C}\right]$ raclopride binding compared to controls, indicative of greater amphetamine-induced DA release. Furthermore, the study did not find differences in amphetamine effects on $\left[{ }^{11} \mathrm{C}\right]$ raclopride binding between medication-free $(\mathrm{N}=5)$ and medication-naïve $(\mathrm{N}=6)$ patients with schizophrenia, indicating that the effect occurs independent of earlier treatment with neuroleptics. Abi-Dargham and colleagues [35] have since then replicated these findings. Furthermore, it was shown that changes in amphetamine-induced dopamine release in schizophrenia might be associated with illness phase, as amphetamine-induced transient worsening of psychotic symptoms correlated with changes in [ $\left.{ }^{1}{ }^{1} \mathrm{C}\right]$ raclopride binding in patients with schizophrenia and no difference in $\left[{ }^{1} \mathrm{C}\right]$ raclopride binding was seen in patients in remission compared to controls [36]. The amphetamine paradigm shows stimulated release, as amphetamine increases dopamine levels by reversing DAT. Another aspect of DA transmission is uncovered with depletion paradigms: The administration of alpha-methyl-para-tyrosine ( $\alpha M P T)$, a reversible competitive inhibitor of tyrosine hydroxylase, causes acute depletion of endogenous DA and can be used as a method to assess the degree of baseline (non-stimulated) intrasynaptic DA activity [37], most likely reflecting basal release as a result of neuronal firing. Abi-Dargham and colleagues [38] used this technique in combination 
with SPECT and [123|]IBZM in 18 untreated patients with schizophrenia and 18 matched control participants before and after AMPT-induced DA depletion. Exposure to aMPT led to increased D2 receptor availability in both patients with schizophrenia and healthy controls, however, the effect was larger in schizophrenia patients, indicative of higher baseline DA activity in this group. The magnitude of the effect did not differ between medication-naïve patients with schizophrenia and those, previously treated with antipsychotics [38]. Similar results were recently obtained by Kegeles and colleagues [39], who employed the same depletion paradigm in combination with PET and [ ${ }^{1} \mathrm{C}$ C]raclopride [39]. Kegeles and colleagues furthermore showed that the largest effect of acute DA depletion on D2 receptor binding was observed in the associative striatum and not in limbic striatum as was long hypothesized. Recently it was shown that amphetamine-stimulated DA release and baseline DA activity are related in patients with schizophrenia but not in controls [40]. Pilot data from Canada furthermore suggest that DA release in patients with schizophrenia and individuals at high risk compared to healthy controls is also increased in response to psychosocial stress [41]. Altogether, studies have consistently demonstrated increased amphetamine-stimulated DA release in schizophrenia. Depletion studies further suggest that schizophrenia is characterized by increased baseline DA activity.

Dopamine reuptake. DA transmission in the striatum is regulated by DA transporters (DAT), which are located on the presynaptic membrane of DA terminals and rapidly remove DA from the synaptic cleft [42]. Several studies have investigated DAT density in schizophrenia, using PET or SPECT, to obtain an index of density of DA terminals and striatal innervation [43-54]. The majority of studies did not observe differences in DAT availability between neuroleptic-naïve patients with schizophrenia and controls [43, 45, 46, 49, 51-53]. Two studies reported reduced DAT binding in patients with schizophrenia compared to controls $[47,50]$. However, since the patients were not medication-naive, the researchers concluded that the observed reduction in DAT binding was secondary to prior treatment with neuroleptics. In order to clarify this issue, Mateos and colleagues [48] repeated the experiment in a cohort of neuroleptic-naïve patients with schizophrenia and also found lower DAT density [48]. Still, this remains the only study, which reported decreased DAT binding in schizophrenia. So, taken together the evidence suggests that schizophrenia is not associated with changes in DAT density. Recently, two dualisotope SPECT studies used [ $\left.{ }^{99} \mathrm{mTc}\right]$ TRODAT and [123|]IBZM to simultaneously measure DAT and D2 receptor availability in drug-naïve patients with schizophrenia and controls $[44,54]$. In line with most of the previous findings, no overall differences in DAT or D2 receptor availability were observed. However, in patients but not controls, DAT density correlated positively with D2 receptor availability [54], which 
was most pronounced in patients presenting with predominantly positive symptoms [44].

\section{Postsynaptic DAergic parameters}

DA receptor availability. The most extensively studied receptor in schizophrenia is the D2 receptor, which is abundantly present in the human striatum. Numerous studies have measured the density of striatal D2 receptors in schizophrenia, both in vivo and postmortem, and three meta-analyses have reviewed the strength and consistency of the reported findings $[14,55,56]$. The first of these meta-analyses was conducted by Zakzanis and Hansen [56] and included 17 studies of which 7 were postmortem. The results from 511 patients with schizophrenia and 534 controls suggested that, although D2 receptor availability was elevated in approximately $70 \%$ of the patients, increased receptor density failed to discriminate patients from controls and is therefore unlikely to represent a general marker of schizophrenia [56]. In line with this are the findings of the second meta-analyses, which included 15 in vivo studies on D2 receptor density in schizophrenia, and concluded that there was small but significant elevation of receptor availability along with greater variability in schizophrenia patients compared to controls [14]. The more recent, comprehensive meta-analysis by Kestler and colleagues [55] included 20 postmortem studies and 17 in vivo studies on D2 receptor availability in schizophrenia and took into account differences in methodology such as in vivo versus postmortem measures (which are likely to be confounded by prior treatment with neuroleptics, which results in upregulation of D2 when administered chronically) as well as the possible influence of sample characteristics such as age, gender or medication status. The authors concluded that the data were compatible with the idea of a subgroup of patients with schizophrenia being characterized by elevated D2 density [55]. The results of a recent study using the high affinity D2/D3 ligand $\left[{ }^{18} \mathrm{~F}\right]$ fallypride were also in line with this, showing selective alterations in D2 density in schizophrenia patients [57]. Several lines of research indicate that, in addition to the D2 receptor, the D3 receptor might play an important role in the pathophysiology of schizophrenia [5860]. However, until recently it was not feasible to selectively target D3 receptors with neurochemical imaging to obtain a direct measure of D3 in schizophrenia, since the available radiotracers exhibited similar affinities for the D2 and D3 receptors and therefore could not distinguish between them. Using the D2/3 agonist radiotracer $\left[{ }^{11} \mathrm{C}\right] \mathrm{PHNO}$, which has higher affinity for D3 than for D2, a recent study did not reveal differences in receptor occupancy between patients with schizophrenia compared to controls [61], although a more selective tracer would be needed to replicate this initial finding. Several studies have used ligand subtraction methods to measure the distribution of striatal D4 receptors in schizophrenia. However, these studies produced inconsistent results, some reporting increased availability of D4 [62-65], while others did not find differences in D4 between 
schizophrenia patients and controls [66-68]. More selective tracers are needed to better characterize these receptors. Most (postmortem) studies did not find altered levels of D1 receptors in the striatum [69-74], one reported a slight decrease in D1 density [75]. The results of two in vivo studies of striatal DI density in schizophrenia are in line with the majority of postmortem findings and do not suggest alterations in striatal D1 receptor levels in schizophrenia $[76,77]$. Taken together, it seems that a subgroup of schizophrenia patients is characterized by increased density of D2-like (i.e. D2/D3) receptors, independent of age, gender and prior neuroleptic exposure. The density of striatal D1 receptors on the other hand seems to be unaltered. No conclusive data is available for D4.

Balance in D2 receptor affinity states. Being the primary target of antipsychotic medication, the D2 receptor plays a major role in schizophrenia and psychosis [78, 79], although, as discussed above, its involvement in the pathophysiology of psychotic disorders remains unclear $[14,55,56]$. The D2 receptor has been shown to exist in two different affinity states: a high affinity, active state (D2 ${ }^{\text {high }}$ ) and a low affinity, inert state (D2 $\left.{ }^{\text {low }}\right)[80,81]$. Preclinical work has shown that radiolabelled DA agonists such as (+)-PHNO, which selectively binds to D2/3 receptors in the highaffinity state, in combination with PET can be used to study the distribution of D2 ${ }^{\text {high }}$ both in-vitro [82] and in-vivo [83, 84]. The distribution of D2high using PET and [ $\left.{ }^{11} \mathrm{C}\right]-$ $(+)$-PHNO was recently demonstrated also in human volunteers [81, 85]. For schizophrenia, it has been suggested that the observed super-sensitivity to DAenhancing drugs such as amphetamine is the result of an imbalance in D2 affinity states, in particular elevated availability of D2 high receptors $[86,87]$. However, evidence for this primarily stems from animal models of schizophrenia [for a review see 86]. Recently, the distribution of D2high was for the first time studied in patients with schizophrenia, and contrary to what could be expected from pre-clinical work, no elevation in D2 ${ }^{\text {high }}$ was observed in medication-free patients with schizophrenia compared to controls [61]. Although the authors acknowledge that differences in D2 ${ }^{\text {high }}$ between schizophrenia patients and controls could have been masked by endogenous DA, to date, there is no evidence for D2high dysregulation in schizophrenia.

\section{Extrastriatal DA alterations}

Several studies have now used high affinity radiotracers such as [ $\left.{ }^{18} \mathrm{~F}\right]$ fallypride, [11C]FLB 457, and [123|]epidepride, to measure the distribution of D2 receptors in low-density brain regions such as the thalamus, anterior cingulate cortex, temporal cortex or substantia nigra, in unmedicated and medication-naïve patients with schizophrenia. Some studies found decreased D2 receptor availability in the thalamus [88-92], anterior cingulate cortex [91, 93], temporal cortex [91, 94] and midbrain [95], while some found no differences in the thalamus [95], anterior 
cingulate and temporal cortex $[88,92,96]$, and one study found an increase in D2 receptor availability in schizophrenia in the substantia nigra [92]. A very large recent study did not confirm any of the above reported extrastriatal D2 receptor alterations in schizophrenia [57].

Another recent study has used the radiotracer $\left[{ }^{1} \mathrm{C}\right] \mathrm{CE} 21$ in combination with PET to visualize thalamic DAT in patients with schizophrenia, who were either medicationnaive or off medication for at least six months [97]. In contrast to striatal brain regions, where DAT seems to be unaffected in schizophrenia, this study reported increased DAT binding in the thalamus of patients with schizophrenia compared to controls. Another study measured extrastriatal DA synthesis capacity using PET and $\left[{ }^{1} \mathrm{C}\right] \mathrm{L}-\mathrm{DOPA}$ and found no differences between medication-naïve patients with schizophrenia and controls with regard to DA synthesis capacity in the thalamus or anterior cingulate and temporal cortex [20], although the ability of this tracer to measure extrastriatal dopamine synthesis is questionable [98].

\section{Prefrontal cortical DA alterations}

While D2 receptors are abundantly present in striatal regions of the brain, the predominant DA receptors in prefrontal cortical regions are of the D1 type [99]. The distribution of D1 receptors in schizophrenia has been studied using the PET radiotracers [ $\left.{ }^{1} \mathrm{C}\right] \mathrm{NNC} 112$ or $\left[{ }^{11} \mathrm{C}\right] \mathrm{SCH} 23390$. However, the few studies that have been conducted produced conflicting results. While Okubo and colleagues [76], using $\left[{ }^{1} \mathrm{C}\right] \mathrm{SCH}$ 23390, found a decrease in receptor binding in patients with schizophrenia compared to controls, the study by Karlsson and colleagues [100], using the same radiotracer, did not reveal any differences between groups [76, 100]. However, patients in this latter study were all drug-naïve, while the former study also included drug-free patients. The more recent study by Hirvonen and colleagues [101] reported decreased [ $\left.{ }^{11} \mathrm{C}\right] \mathrm{SCH} 23390$ binding in frontotemporal brain regions of previously medicated patients with schizophrenia compared to their unaffected co-twins and healthy comparison twins and higher doses of antipsychotics were associated with greater decreases in D1 receptor binding [101]. Interestingly, unaffected monozygotic co-twins displayed increased receptor binding compared to healthy comparison twins, and unaffected dizygotic co-twins showed intermediate levels. Two other studies used the radiotracer $\left[{ }^{11} \mathrm{C}\right] N N C$ 112: One reported increased D1 receptor binding in the schizophrenia group, which correlated with deficits in working memory performance [77], and the second study found increases limited to the drug naïve patients but not the previously treated ones [102]. Studies on D1 receptor availability in schizophrenia are summarized in table 1. 
Table 1. PET studies on D1 receptor availability in PFC in schizophrenia

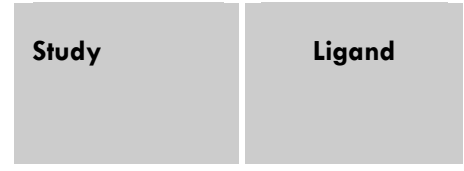

Okubo et al., 1997 $\left[{ }^{1} \mathrm{C}\right] \mathrm{SCH} 23390$

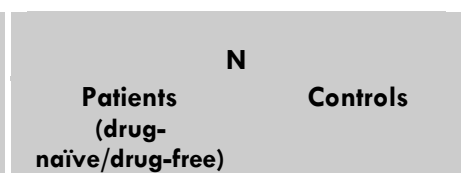

18
$17(10 / 7)$

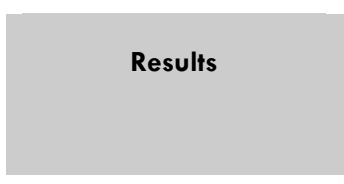

Decreased receptor binding in patients versus controls

\section{Karlsson et al., 2002}

Hirvonen et al., $\quad\left[{ }^{11} \mathrm{C}\right] \mathrm{SCH} 23390$ 2006

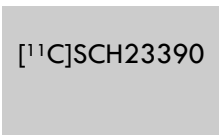

$9(0 / 9)$
$10(10 / 0)$
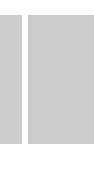

10

24
No difference between patients and controls
Decreased receptor

binding in patients versus controls (i.e. their unaffected co-twins and healthy comparison twins), but increased receptor binding in unaffected co-twins versus healthy comparison twins

\begin{tabular}{|c|c|c|c|c|}
\hline $\begin{array}{l}\text { Abi-Dargham } \\
\text { et al., } 2002\end{array}$ & {$\left[{ }^{11} \mathrm{C}\right] \mathrm{NNC} 112$} & $16(9 / 7)$ & 16 & $\begin{array}{l}\text { Increased receptor } \\
\text { binding in patients ver } \\
\text { controls }\end{array}$ \\
\hline $\begin{array}{l}\text { Abi-Dargham } \\
\text { et al., } \\
\text { in press }\end{array}$ & {$\left[{ }^{11} \mathrm{C}\right] \mathrm{NNC} 112$} & $25(12 / 13)$ & 40 & $\begin{array}{l}\text { Increased receptor } \\
\text { binding only in drug- } \\
\text { naive patients versus } \\
\text { controls; no change in } \\
\text { drug-free patients }\end{array}$ \\
\hline
\end{tabular}

While it is possible that the discrepancy in findings is due to the influence of neuroleptic medication, it might also be that differences in radioligand properties contributed to the diverging results, as shown by Guo and colleagues [103]: Using a DA depletion paradigm the researchers could demonstrate that the in vivo binding of the two radiotracers $\left[{ }^{1} \mathrm{C}\right] \mathrm{SCH} 23390$ and $\left[{ }^{1} \mathrm{C}\right] \mathrm{NNC} 112$ was affected differentially by changes in endogenous DA, indicating that the increased DI receptor availability observed in the studies by Abi-Dargham and colleagues using [11C]NNC 112 might reflect an upregulation of D1 receptors, secondary to chronically low DA levels. This would also be consistent with the observed correlation between increased DI receptor binding and deficits in working memory [77]. However, since both radioligands also bind to the serotonergic $5-\mathrm{HT}_{2 \mathrm{~A}}$ receptor 
$[104,105]$, selective tracers are needed to pursue these investigations of the role of cortical D1 receptors in schizophrenia.

\section{Dopamine in at-risk and prodromal states}

The above described studies have revealed several aspects of DA dysfunction in patients with established schizophrenia. In order to extend these findings and shed light on the timing of DA dysfunction in schizophrenia, researchers have started recently to examine different aspects of DA transmission in individuals with prodromal signs of schizophrenia and individuals at genetic or psychometric risk for psychosis.

Three studies have investigated striatal DA synthesis capacity with PET and [ $\left.{ }^{18} \mathrm{~F}\right] \mathrm{F}$ DOPA. While Huttunen and colleagues [106] observed increased [18F]F-DOPA uptake in the caudate-putamen in low-symptomatic first-degree relatives of patients with schizophrenia compared to controls, the study by Shotbolt and colleagues [29] did not reveal any changes in radiotracer uptake in unaffected and completely asymptomatic co-twins of schizophrenia patients compared to controls $[29,106]$. Howes and colleagues [24] found increased [ $\left.{ }^{18} \mathrm{~F}\right]-\mathrm{DOPA}$ uptake in individuals with prodromal signs of schizophrenia compared to controls [24]. Interestingly, this effect was most pronounced in the associative striatum and correlated positively with severity of prodromal symptoms. Moreover, in a subsequent study the authors could indicate that the increase in DA synthesis capacity observed in individuals in the prodromal phase of the illness progressed over time only in those individuals who later developed schizophrenia, but not in those who did not develop the disease [107]. However, in contrast to the findings in prodromal individuals, where the effect was most pronounced in associative striatum, the progression in DA synthesis alteration in those who developed the disease was only seen in sensorimotor striatum [107]. Three other studies have examined stimulated DA release in individuals psychometrically at-risk for schizophrenia (i.e. individuals with schizoptypal traits). Soliman and colleagues [108] employed a psychosocial stress paradigm in combination with PET and [11C]raclopride to study stress-induced DA release in psychometric schizotypes compared to controls. No changes in DA release in response to stress were observed in normal controls, nor in the 'positive' schizotypes (i.e. individuals with perceptual aberrations). Only the 'negative' schizotypes (i.e. individuals with physical anhedonia) showed an increase in DA release in response to stress compared to baseline [108]. Abi-Dargham and colleagues [109] as well as Woodward and colleagues [110] studied amphetamine-induced DA release in individuals with schizotypal personality disorder (SPD) and individuals with schizotypal traits, respectively. In the SPD subjects of the first study, amphetamine caused a larger decrease in [123/]IBZM binding compared to controls [109]. Similarly, amphetamine-induced DA release measured with PET and [18F]fallypride 
correlated positively with schizotypal traits in the second study [1 10]. In accordance with previous findings showing that DA dysregulation might be most pronounced in associated striatum as opposed to limbic or sensorimotor regions [24, 39], the correlation between stimulated DA release and schizotypal traits was strongest in associative striatum. Finally, one study has investigated striatal D2 receptor availability in six monozygotic and 5 dizygotic unaffected co-twins of patients with schizophrenia and compared them to control twins without a family history of psychosis. Elevated caudate D2 receptor availability was observed only in the monozygotic co-twins of schizophrenia patients, compared to dizygotic co-twins and controls. No changes however were revealed in the dizygotic co-twins compared to controls [ $\left[\begin{array}{lll}1 & 1 & 1\end{array}\right]$.

In summary, studies suggest an increase in DA in schizotypal states and in relation to schizotypal and prodromal symptoms, linking DA dysfunction to the expression of the schizophrenia phenotype. Combined with the findings of Shotbolt and colleagues who did not observe any changes in DA synthesis capacity in unaffected co-twins of patients with schizophrenia [29], this suggests that DA dysfunction does not relate to a general genetic vulnerability in the absence of behavioral manifestations but rather represents a biological marker for the very early expression of schizophrenia symptomatology.

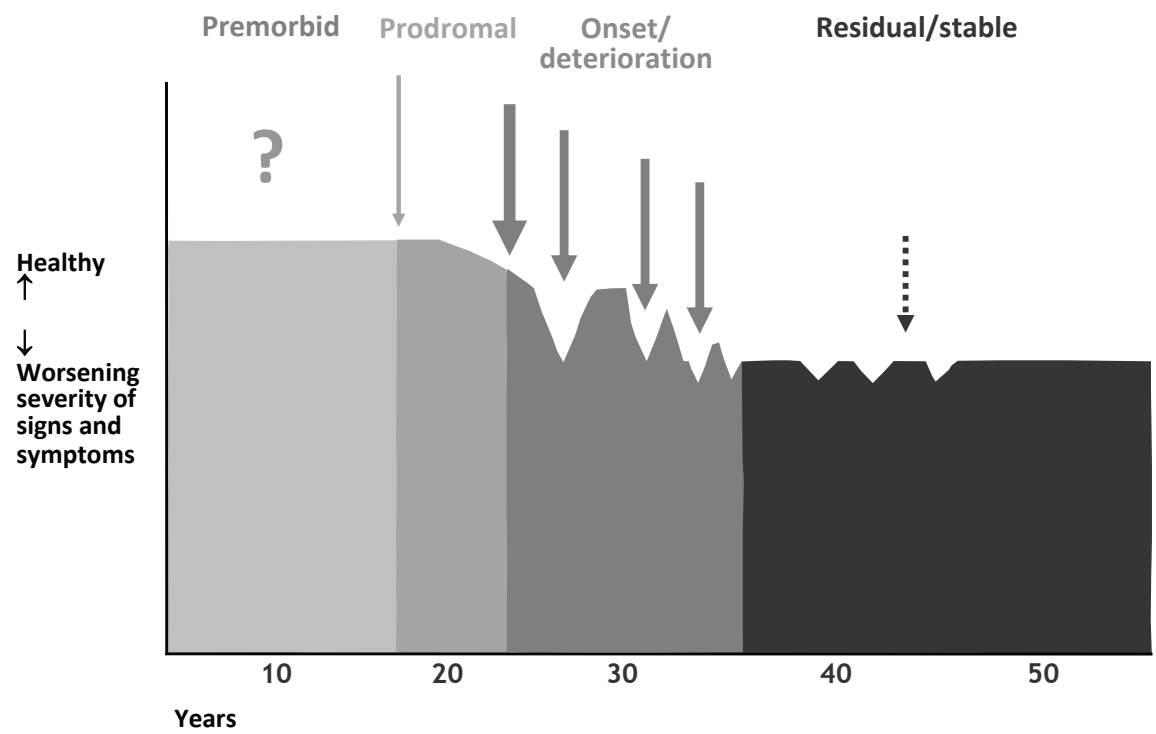

Figure 1. Schematic illustration of the course of the DA deficit in schizophrenia. DA dysregulation has been shown to occur already in the prodromal phase of the illness and seems to progress along with illness progression, peaking at illness onset. Adapted with permission from Lieberman [ 1112$]$. 


\section{Summary}

The most consistently found dopaminergic alteration in schizophrenia is elevated presynaptic DA function in striatal brain regions (see figures 2 and 3 for an illustration), in particular associative striatum, as demonstrated by imaging studies showing i) increased L- DOPA uptake as an index of increased DA synthesis capacity, ii) elevated amphetamine-induced DA release and iii) elevated D2 occupancy by DA as revealed by the acute DA depletion. DAT seems to be unaffected in schizophrenia, and increased D2 availability was only found in a subgroup of patients. Findings concerning the distribution of D3 and D4 remain understudied and equivocal. Although preclinical work suggests an imbalance in D2 receptor affinity states being associated with psychosis, the only study in patients with schizophrenia did not find elevated D2 ${ }^{\text {high. }}$. The evidence concerning DA alterations in extrastriatal and prefrontal cortical brain areas in schizophrenia seems less consistent. While some studies found elevated levels of D2 in areas such as the thalamus, anterior cingulate and temporal cortex or the midbrain and substantia nigra, others did not. The results of the few studies on D1 receptor availability in prefrontal cortex in schizophrenia are also conflicting. Thus, while a lot of effort has been put into the investigation of striatal DA alterations in schizophrenia, research has been less successful with regard to extrastriatal and prefrontal cortical regions. More research is needed to resolve the inconsistencies in findings research has provided so far.

\section{Functional and clinical implications}

As defined in the current, fourth version of the Diagnostic and Statistical Manual of Mental Disorders [113], schizophrenia is a highly heterogeneous disease, presenting with positive, negative and cognitive symptoms. Positive symptoms include hallucinations and delusions, while negative symptoms refer to flattened affect, anhedonia and loss of motivation. Cognitive disturbances are mostly seen in domains such as working memory, executive function and aspects of social cognition. DA dysregulation plays a role within each of these dimensions. While positive symptoms seem to most directly relate to excessive striatal DA transmission, negative and cognitive symptoms have been associated with decreased DA function in PFC. The latter assumption was primarily based on the known and crucial involvement of the PFC in cognitive and emotional processes [1 $114-116]$. Still, after decades of research into DA and schizophrenia, it remains elusive how DA dysregulation actually translates into the complex and multifactorial symptoms that characterize the clinical picture of schizophrenia. 


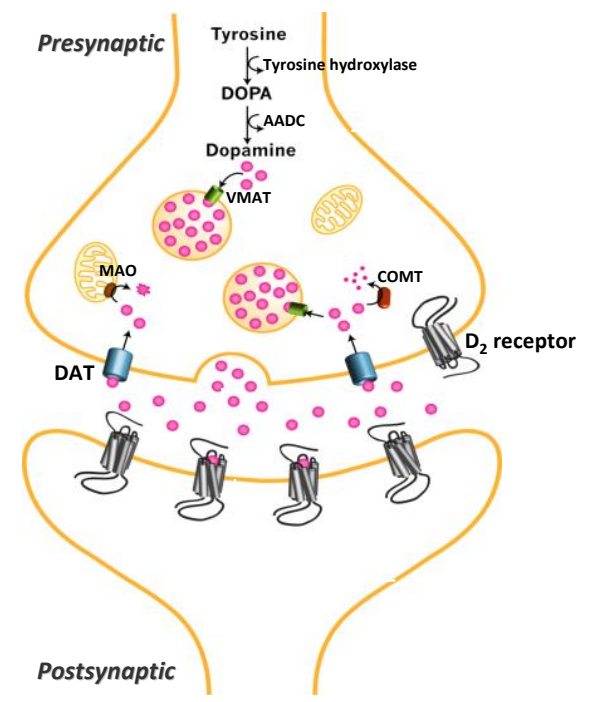

Figure 2. DA transmission at striatal medium spiny neurons. The most consistently found dysregulation in schizophrenia points to increased DA synthesis, increased stimulated DA release and increased D2 receptor binding after acute DA depletion.

The DA hypothesis:

Excess DA in mesolimbic pathways - VST

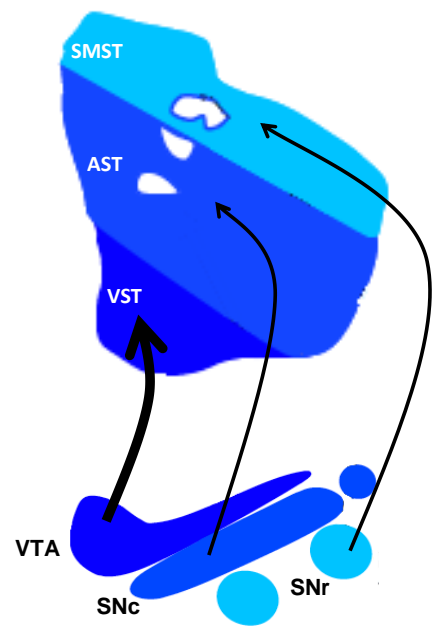

The new evidence:

Excess DA in nigrostriatal pathways - AST

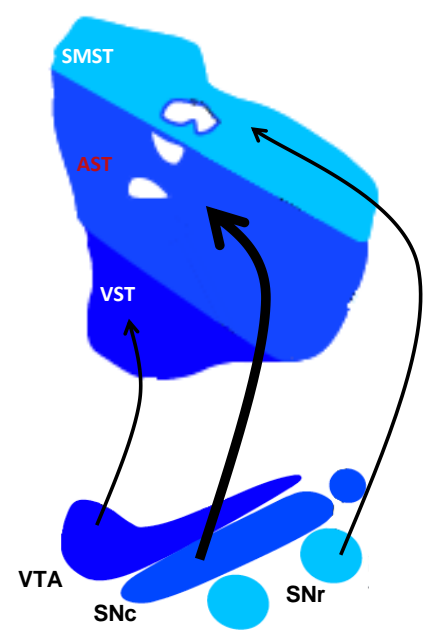

Figure 3. New data bringing new evidence: The DA dysfunction in schizophrenia seems to be most pronounced in AST as opposed to VST as previously assumed. Adapted with permission from Simpson and colleagues [1 17]. SMST (sensorimotor striatum), AST (associative striatum), VST (ventral striatum), VTA (ventral tegmental area), $\mathrm{SNc}$ (substantia nigra pars compacta), $\mathrm{SNr}$ (substantia nigra pars reticulate). 


\section{Relating DA dysfunction to positive symptoms}

Several of the imaging studies discussed in this chapter, have reported associations between alterations in striatal DA function and symptomatology in patients with schizophrenia. Regarding DA synthesis, Hietala and colleagues found a negative correlation between striatal F-DOPA uptake and depressive symptoms and a positive correlation with paranoid symptoms, although this was significant at trendlevel only [22]. Howes and colleagues reported a positive correlation between severity of positive prodromal symptoms as well as neuropsychological impairment and increased DA synthesis capacity in associative striatum. However, this was not true for depressive symptoms [24]. With regard to DA release, Laruelle and colleagues [33] found that amphetamine-induced decrease in D2 binding potential was associated with positive symptoms. In a subsequent study the authors could furthermore establish a relation between amphetamine-stimulated DA release and illness-phase, as amphetamine-stimulated DA release was only increased in patients presenting with acute schizophrenia but not in patients in remission [36]. Recently, Woodward and colleagues revealed a positive correlation between stimulated DA release and schizoptypal personality traits in healthy individuals [1 10$]$.

Assuming that striatal hyperdopaminergia plays a role in the emergence and experience of positive psychotic symptoms, the question arises as to how dopaminergic alterations in striatal brain regions ultimately give rise to the experience of hallucinations and delusions. Altered salience attribution has been suggested as a possible mechanism [118]. Burst firing of dopamine neurons in the ventral tegmental area markedly increases dopamine release in the striatum [119] and is believed to mediate the perception of salience or reward associated with stimuli [120-122]. The phasic bursts of dopamine release, which are highly dependent on glutamatergic excitatory afferents, have been shown to be regulated by constant low-frequency tonic firing of dopamine neurons [42]. Tonic dopamine tone in turn is under control of GABAergic inhibition. Increased levels of tonic dopamine firing may result in decreased amplitude of phasic dopamine burst firing, thus dampening responsivity of this system. Decreased tonic dopamine levels, on the other hand, may result in a heightened responsivity of the phasic dopamine component [42, 123]. Kapur has suggested that, in schizophrenia, dopamine dysregulation results in a psychological state of aberrant salience, in which mundane events and ideas may be attributed with undue significance [118]. Thus, a hyperdopaminergic state in striatal brain regions, which most likely reflects dysregulation of the phasic component of DA release [124, 125], is believed to create a condition in which logically unconnected ideas and associations are weaved together and elaborated upon, eventually leading to the emergence of a delusional system. The process of salience attribution has been related to associative and reinforcement learning, in which what is called "reward prediction error" plays a key 
role [126]. It is hypothesized that previous reward outcomes are used to form a reward prediction, which is then compared to the actual current reward. The difference between reward prediction and actual outcome is referred to as the reward prediction error [126], and has been shown to be mediated, in animals as well as in humans, by dopamine activity in ventral midbrain and striatum [127-130]. Compared to healthy controls, patients with psychosis seem to exhibit aberrant reward prediction and reward-related learning, both at the behavioral and the neural level $[131,132]$. However, the relationship between dopamine dysfunction in these brain regions, alterations in reward processing and symptomatology in schizophrenia has not directly been studied and remains speculative.

\section{Relating DA dysfunction to negative and cognitive symptoms}

As intact dopaminergic neurotransmission is critical for PFC functioning and cognition, $[114,133]$, the negative and cognitive symptoms of schizophrenia have been particularly associated with cortical hypodopaminergia [77, 134, 135], although direct evidence for this association in schizophrenia is missing. The nature of dopamine dysfunction in the cortex remains unclear, although one study showing decreased tyrosine hydroxylase immunolabeling suggests decreased innervation [136]. Recently, the group of Simpson, Kellendonk and Kandel and colleagues suggested a role for the striatum in the etiology of negative and cognitive symptoms of schizophrenia [117]. Based on their preclinical work in D2 overexpressing mice, the researchers demonstrated that striatal DA alterations in form of overexpression of D2 leads to changes in DA turnover and prefrontal D1 receptor stimulation [137]. Behaviorally, this was accompanied by deficits in working memory [137] and operant performance, expressed in both reduced motivation and deficits in timing of the rewards [138]. It was moreover shown that the deficits in cognitive performance were secondary to the motivational deficit directly resulting from the D2 overexpression [139], and remained even after the D2 overexpression had been reversed [138]. In line with these preclinical findings, studies in individuals with prodromal symptoms of schizophrenia have revealed an association between increased striatal DA synthesis capacity and altered activation in prefrontal cortical brain regions during cognitive engagement [140, 141].

\section{Conclusions}

This chapter reviewed evidence for DA dysfunction in schizophrenia. Most of this evidence stems form imaging studies applying PET and SPECT techniques. Due to advances in these imaging techniques it has become possible to study neurochemical alterations in the DA system in several regions of the brain. Accordingly, recent studies were able to examine DA neurotransmission in the different substructures of 
the striatum and revealed that, in contrast to the prevailing idea of DA hyperactivity in the mesolimbic DA pathway, it is rather hyperactivity in associative striatum that is implicated in schizophrenia pathology, as illustrated in figure 3. It further seems that the dysfunction is presynaptic rather than postsynaptic, characterized by increased DA synthesis capacity and increased phasic release to pharmacological and possibly also psychosocial challenges (see figure 2). Some of these alterations have been demonstrated also in individuals with prodromal signs of schizophrenia and seem to progress along with illness progression, suggesting that the dysfunction occurs early in the disease and may represent an early pathogenic process leading to further dysregulation (for an illustration on the course of the DA deficit see figure 1).

Despite recent advances in the study of DA dysregulation in schizophrenia, the etiology of DA imbalance remains unknown. It has been assumed that striatal DA hyperactivity results from decreased activity in PFC, due to its functional role in inhibiting subcortical DA transmission [26, 142]. Conversely, preclinical work has recently shown that striatal DA abnormalities result in altered PFC DA activity [137]. The striatum is a complex integrative structure, receiving among others input from the hippocampus, an area of pathology in schizophrenia [143, 144], and in animal models changes in hippocampal activity lead to dysregulation of DA neuronal acitvity [145]. Future research should take into account these preclinical observations to understand the circuitry involved in the striatal and cortical dopamine dysfunction in schizophrenia. 


\section{References}

1. Carlsson, A., Does dopamine play a role in schizophrenia? Psychological Medicine, 1977. 7(4): p. 583-97.

2. Carlsson, A., Antipsychotic drugs, neurotransmitters, and schizophrenia. American Journal of Psychiatry, 1978. 135(2): p. 165-73.

3. van Rossum, J.M., The significance of dopamine-receptor blockade for the mechanism of action of neuroleptic drugs. Arch Int Pharmacodyn Ther, 1966. 160(2): p. 492-4.

4. Lieberman, J.A., B.B. Sheitman, and B.J. Kinon, Neurochemical sensitization in the pathophysiology of schizophrenia: deficits and dysfunction in neuronal regulation and plasticity. Neuropsychopharmacology, 1997. 17(4): p. 205-29.

5. Laruelle, M., L.S. Kegeles, and A. Abi-Dargham, Glutamate, Dopamine, and Schizophrenia: From Pathophysiology to Treatment. Ann N Y Acad Sci, 2003. 1003: p. 138-158.

6. Howes, O.D. and S. Kapur, The dopamine hypothesis of schizophrenia: version III--the final common pathway. Schizophrenia Bulletin, 2009. 35(3): p. 549-62.

7. Creese, I., D.R. Burt, and S.H. Snyder, Dopamine receptor binding predicts clinical and pharmacological potencies of antischizophrenic drugs. Science, 1976. 192(4238): p. 481-3.

8. Davis, K.L., et al., Dopamine in schizophrenia: a review and reconceptualization. American Journal of Psychiatry, 1991. 148(11): p. 1474-86.

9. Weinberger, D.R., Implications of normal brain development for the pathogenesis of schizophrenia. Archives of General Psychiatry, 1987. 44(7): p. 660-9.

10. Knable, M.B. and D.R. Weinberger, Dopamine, the prefrontal cortex and schizophrenia. Journal of Psychopharmacology, 1997. 11 (2): p. 123-31.

11. Abi-Dargham, A., Do we still believe in the dopamine hypothesis? New data bring new evidence. International Journal of Neuropsychopharmacology, 2004. 7 Suppl 1: p. S1-5.

12. Erritzoe, D., et al., Positron emission tomography and single photon emission CT molecular imaging in schizophrenia. Neuroimaging Clin N Am, 2003. 13(4): p. 817-32.

13. Seeman, P., et al., Psychosis pathways converge via D2high dopamine receptors. Synapse, 2006. 60(4): p. 319-46.

14. Laruelle, M., Imaging dopamine transmission in schizophrenia. A review and meta-analysis. Quarterly Journal of Nuclear Medicine, 1998. 42(3): p. $211-21$.

15. Brown, W.D., et al., FluoroDOPA PET shows the nondopaminergic as well as dopaminergic destinations of levodopa. Neurology, 1999. 53(6): p. $1212-8$.

16. Cumming, P. and A. Gjedde, Compartmental analysis of dopa decarboxylation in living brain from dynamic positron emission tomograms. Synapse, 1998. 29(1): p. 37-61.

17. Garnett, E.S., G. Firnau, and C. Nahmias, Dopamine visualized in the basal ganglia of living man. Nature, 1983. 305(5930): p. 137-8.

18. Garnett, E.S., et al., [18F]fluoro-dopa, an analogue of dopa, and its use in direct external measurements of storage, degradation, and turnover of intracerebral dopamine. Proc Natl Acad Sci U S A, 1978. 75(1): p. 464-7.

19. Reith, J., et al., Elevated dopa decarboxylase activity in living brain of patients with psychosis. Proc Natl Acad Sci U S A, 1994. 91(24): p. 11651-4.

20. Nozaki, S., et al., Regional dopamine synthesis in patients with schizophrenia using L-[beta-1 1C]DOPA PET. Schizophr Res, 2009. 108(1-3): p. 78-84.

21. Lindstrom, L.H., et al., Increased dopamine synthesis rate in medial prefrontal cortex and striatum in schizophrenia indicated by L-(beta-1 1C) DOPA and PET. Biol Psychiatry, 1999. 46(5): p. 681-8.

22. Hietala, J., et al., Depressive symptoms and presynaptic dopamine function in neuroleptic-naive schizophrenia. Schizophr Res, 1999. 35(1): p. 41-50. 
23. Hietala, J., et al., Presynaptic dopamine function in striatum of neuroleptic-naive schizophrenic patients. Lancet, 1995. 346(8983): p. $1130-1$.

24. Howes, O.D., et al., Elevated striatal dopamine function linked to prodromal signs of schizophrenia. Archives of General Psychiatry, 2009. 66(1): p. 13-20.

25. McGowan, S., et al., Presynaptic dopaminergic dysfunction in schizophrenia: a positron emission tomographic [18F]fluorodopa study. Arch Gen Psychiatry, 2004. 61(2): p. 134-42.

26. Meyer-Lindenberg, A., et al., Reduced prefrontal activity predicts exaggerated striatal dopaminergic function in schizophrenia. Nat Neurosci, 2002. 5(3): p. 267-71.

27. Dao-Castellana, M.H., et al., Presynaptic dopaminergic function in the striatum of schizophrenic patients. Schizophrenia Research, 1997. 23(2): p. 167-74.

28. Elkashef, A.M., et al., 6-(18)F-DOPA PET study in patients with schizophrenia. Positron emission tomography. Psychiatry Research, 2000. 100(1): p. 1-11.

29. Shotbolt, P., et al., Striatal dopamine synthesis capacity in twins discordant for schizophrenia. Psychological Medicine, 201 1: p. 1-8.

30. Laruelle, M., et al., SPECT imaging of striatal dopamine release after amphetamine challenge. J Nucl Med, 1995. 36(7): p. $1182-90$.

31. Kegeles, L.S., et al., Stability of [123I]IBZM SPECT measurement of amphetamine-induced striatal dopamine release in humans. Synapse, 1999. 31(4): p. 302-8.

32. Laruelle, M., Imaging synaptic neurotransmission with in vivo binding competition techniques: a critical review. J Cereb Blood Flow Metab, 2000. 20(3): p. 423-51.

33. Laruelle, M., et al., Single photon emission computerized tomography imaging of amphetamineinduced dopamine release in drug-free schizophrenic subjects. Proc Natl Acad Sci U S A, 1996. 93(17): p. 9235-40.

34. Breier, A., et al., Schizophrenia is associated with elevated amphetamine-induced synaptic dopamine concentrations: evidence from a novel positron emission tomography method. Proceedings of the National Academy of Sciences, 1997. 94(6): p. 2569-74.

35. Abi-Dargham, A., et al., Increased striatal dopamine transmission in schizophrenia: confirmation in a second cohort. American Journal of Psychiatry, 1998. 155(6): p. 761-7.

36. Laruelle, M., et al., Increased dopamine transmission in schizophrenia: relationship to illness phases. Biol Psychiatry, 1999. 46(1): p. 56-72.

37. Laruelle, M., et al., Imaging D2 receptor occupancy by endogenous dopamine in humans. Neuropsychopharmacology, 1997. 17(3): p. 162-74.

38. Abi-Dargham, A., et al., Increased baseline occupancy of D2 receptors by dopamine in schizophrenia. Proceedings of the National Acadademy of Science 2000. 97(14): p. 8104-9.

39. Kegeles, L.S., et al., Increased synaptic dopamine function in associative regions of the striatum in schizophrenia. Arch Gen Psychiatry, 2010. 67(3): p. 231-9.

40. Abi-Dargham, A., et al., Baseline and amphetamine-stimulated dopamine activity are related in drugnaive schizophrenic subjects. Biological Psychiatry, 2009. 65(12): p. 1091-3.

41. Mizrahi, R., Advances in PET analyses of stress and dopamine. Neuropsychopharmacology, 2010. 35(1): p. 348-9.

42. Goto, Y., S. Otani, and A.A. Grace, The Yin and Yang of dopamine release: a new perspective. Neuropharmacology, 2007. 53(5): p. 583-7

43. Yoder, K.K., et al., Dopamine transporter density in schizophrenic subjects with and without tardive dyskinesia. Schizophr Res, 2004. 71(2-3): p. 371-5.

44. Schmitt, G.J., et al., Dual-isotope SPECT imaging of striatal dopamine: first episode, drug naive schizophrenic patients. Schizophr Res, 2008. 101 (1-3): p. 133-41.

45. Schmitt, G.J., et al., The striatal dopamine transporter in first-episode, drug-naive schizophrenic patients: evaluation by the new SPECT-ligand[99mTc]TRODAT-1. J Psychopharmacol, 2005. 19(5): p. 488-93. 
46. Schmitt, G.J., et al., Striatal dopamine transporter availability is associated with the productive psychotic state in first episode, drug-naive schizophrenic patients. Eur Arch Psychiatry Clin Neurosci, 2006. 256(2): p. 115-21.

47. Mateos, J.J., et al., Decreased striatal dopamine transporter binding assessed with [123I] FP-CIT in first-episode schizophrenic patients with and without short-term antipsychotic-induced parkinsonism. Psychopharmacology (Berl), 2005. 181(2): p. $401-6$.

48. Mateos, J.J., et al., Lower striatal dopamine transporter binding in neuroleptic-naive schizophrenic patients is not related to antipsychotic treatment but it suggests an illness trait. Psychopharmacology (Berl), 2007. 191(3): p. 805-11.

49. Laruelle, M., et al., Dopamine and serotonin transporters in patients with schizophrenia: an imaging study with [(123)I]beta-CIT. Biol Psychiatry, 2000. 47(5): p. 371-9.

50. Laakso, A., et al., Decreased striatal dopamine transporter binding in vivo in chronic schizophrenia. Schizophr Res, 2001. 52(1-2): p. 115-20.

51. Laakso, A., et al., Striatal dopamine transporter binding in neuroleptic-naive patients with schizophrenia studied with positron emission tomography. Am J Psychiatry, 2000. 157(2): p. 269-71.

52. Hsiao, M.C., et al., Dopamine transporter change in drug-naive schizophrenia: an imaging study with 99mTc-TRODAT-1. Schizophr Res, 2003. 65(1): p. 39-46.

53. Lavalaye, J., et al., Dopamine transporter density in young patients with schizophrenia assessed with [1 23]FP-CIT SPECT. Schizophrenia Research, 2001. 47(1): p. 59-67.

54. Yang, Y.K., et al., Associated alterations of striatal dopamine D2/D3 receptor and transporter binding in drug-naive patients with schizophrenia: a dual-isotope SPECT study. American Journal of Psychiatry, 2004. 161(8): p. 1496-8.

55. Kestler, L.P., E. Walker, and E.M. Vega, Dopamine receptors in the brains of schizophrenia patients: a meta-analysis of the findings. Behavioural Pharmacology, 2001. 12(5): p. 355-71.

56. Zakzanis, K.K. and K.T. Hansen, Dopamine D2 densities and the schizophrenic brain. Schizophrenia Research, 1998. 32(3): p. 201-6.

57. Kegeles, L.S., et al., Striatal and extrastriatal dopamine D2/D3 receptors in schizophrenia evaluated with [18F]fallypride positron emission tomography. Biological Psychiatry, 2010. 68(7): p. 634-41.

58. Gurevich, E.V., et al., Mesolimbic dopamine D3 receptors and use of antipsychotics in patients with schizophrenia. A postmortem study. Arch Gen Psychiatry, 1997. 54(3): p. 225-32.

59. Griffon, N., et al., The dopamine D3 receptor and schizophrenia: pharmacological, anatomical and genetic approaches. European Neuropsychopharmacology, 1995. 5 Suppl: p. 3-9.

60. Sokoloff, P., et al., The dopamine D3 receptor: a therapeutic target for the treatment of neuropsychiatric disorders. CNS \& Neurological Disorders Drug Targets, 2006. 5(1): p. 25-43.

61. Graff-Guerrero, A., et al., The dopamine D2 receptors in high-affinity state and D3 receptors in schizophrenia: a clinical [11C]-(+)-PHNO PET study. Neuropsychopharmacology, 2009. 34(4): p. 1078-86.

62. Marzella, P.L., et al., The binding of both $[3 \mathrm{H}]$ nemonapride and $[3 \mathrm{H}]$ raclopride is increased in schizophrenia. Biological Psychiatry, 1997. 42(8): p. 648-54.

63. Murray, A.M., et al., Distribution of putative D4 dopamine receptors in postmortem striatum from patients with schizophrenia. Journal of Neuroscience, 1995. 15(3 P+ 2): p. 2186-91.

64. Seeman, P., H.C. Guan, and H.H. Van Tol, Dopamine D4 receptors elevated in schizophrenia. Nature, 1993. 365(6445): p. $441-5$.

65. Sumiyoshi, T., et al., Dopamine D4 receptors and effects of guanine nucleotides on [3H]raclopride binding in postmortem caudate nucleus of subjects with schizophrenia or major depression. Brain Research, 1995. 681(1-2): p. 109-16.

66. Lahti, R.A., et al., D2-type dopamine receptors in postmortem human brain sections from normal and schizophrenic subjects. Neuroreport, 1996. 7(12): p. 1945-8.

67. Lahti, R.A., et al., Direct determination of dopamine D4 receptors in normal and schizophrenic postmortem brain tissue: a [3H]NGD-94-1 study. Molecular Psychiatry, 1998. 3(6): p. 528-33. 
68. Reynolds, G.P. and S.L. Mason, Are striatal dopamine D4 receptors increased in schizophrenia? Journal of Neurochemistry, 1994. 63(4): p. 1576-7.

69. Joyce, J.N., et al., Organization of dopamine D1 and D2 receptors in human striatum: receptor autoradiographic studies in Huntington's disease and schizophrenia. Synapse, 1988. 2(5): p. 546-57.

70. Pimoule, C., et al., [3H]SCH 23390 labeled D1 dopamine receptors are unchanged in schizophrenia and Parkinson's disease. European Journal of Pharmacology, 1985. 114(2): p. 235-7.

71. Reynolds, G.P. and C. Czudek, Status of the dopaminergic system in post-mortem brain in schizophrenia. Psychopharmacology Bulletin, 1988. 24(3): p. 345-7.

72. Seeman, P., et al., Human brain D1 and D2 dopamine receptors in schizophrenia, Alzheimer's, Parkinson's, and Huntington's diseases. Neuropsychopharmacology, 1987. 1(1): p. 5-15.

73. Knable, M.B., et al., Quantitative autoradiography of dopamine-D1 receptors, D2 receptors, and dopamine uptake sites in postmortem striatal specimens from schizophrenic patients. BiologicalPsychiatry, 1994. 36(12): p. 827-35.

74. Cross, A.J., T.J. Crow, and F. Owen, 3H-Flupenthixol binding in post-mortem brains of schizophrenics: evidence for a selective increase in dopamine D2 receptors. Psychopharmacology (Berl), 1981. 74(2): p. 122-4.

75. Hess, E.J., et al., Dopamine receptor subtype imbalance in schizophrenia. Life Sciences, 1987. 40(15): p. 1487-97.

76. Okubo, Y., et al., Decreased prefrontal dopamine D1 receptors in schizophrenia revealed by PET. Nature, 1997. 385(6617): p. 634-6.

77. Abi-Dargham, A., et al., Prefrontal dopamine D1 receptors and working memory in schizophrenia. Journal of Neuroscience, 2002. 22(9): p. 3708-19.

78. Kapur, S. and D. Mamo, Half a century of antipsychotics and still a central role for dopamine D2 receptors. Progress in Neuropsychopharmacology \& Biologial Psychiatry, 2003. 27(7): p. 1081-90.

79. Kapur, S. and G. Remington, Dopamine $D(2)$ receptors and their role in atypical antipsychotic action: still necessary and may even be sufficient. Biologial Psychiatry, 2001. 50(11): p. 873-83.

80. Sibley, D.R., A. De Lean, and I. Creese, Anterior pituitary dopamine receptors. Demonstration of interconvertible high and low affinity states of the D-2 dopamine receptor. Journal of Biological Chemistry, 1982. 257(11): p. 6351-61.

81. Willeit, M., et al., High-affinity states of human brain dopamine $D 2 / 3$ receptors imaged by the agonist [1 1C]-(+)-PHNO. Biologial Psychiatry, 2006. 59(5): p. 389-94.

82. Nobrega, J.N. and P. Seeman, Dopamine D2 receptors mapped in rat brain with $[3 H](+) P H N O$. Synapse, 1994. 17(3): p. 167-72.

83. Ginovart, N., et al., Binding characteristics and sensitivity to endogenous dopamine of [1 1C]-(+)$P H N O$, a new agonist radiotracer for imaging the high-affinity state of D2 receptors in vivo using positron emission tomography. Journal of Neurochemistry, 2006. 97(4): p. 1089-103.

84. Galineau, L., et al., In vivo characterization of the pharmacokinetics and pharmacological properties of $[11 \mathrm{C}]-(+)-$ PHNO in rats using an intracerebral beta-sensitive system. Synapse, 2006. 60(2): $p$. 172-83.

85. Graff-Guerrero, A., et al., Brain region binding of the D2/3 agonist [ $11 \mathrm{C}]-(+)-P H N O$ and the D2/3 antagonist [ 1 1C]raclopride in healthy humans. Human Brain Mapping, 2008. 29(4): p. 400-10.

86. Seeman, P., All Roads to Schizophrenia Lead to Dopamine Supersensitivity and Elevated Dopamine D2 Receptors. CNS Neurosci Ther, 2010.

87. Seeman, P., et al., Dopamine supersensitivity correlates with D2High states, implying many paths to psychosis. Proceedings of the National Academy of Sciences, 2005. 102(9): p. 3513-8.

88. Talvik, M., et al., Decreased thalamic D2/D3 receptor binding in drug-naive patients with schizophrenia: a PET study with [1 1C]FLB 457. International Journal of Neuropsychopharmacology, 2003. 6(4): p. $361-70$.

89. Yasuno, F., et al., Low dopamine d(2) receptor binding in subregions of the thalamus in schizophrenia. Amerian Journal of Psychiatry, 2004. 161(6): p. 1016-22. 
90. Talvik, M., et al., Dopamine D2 receptor binding in drug-naive patients with schizophrenia examined with raclopride-C11 and positron emission tomography. Psychiatry Research, 2006. 148(2-3): p. 165-73.

91. Buchsbaum, M.S., et al., D2/D3 dopamine receptor binding with [F-18]fallypride in thalamus and cortex of patients with schizophrenia. Schizophrenia Research, 2006. 85(1-3): p. 232-44.

92. Kessler, R.M., et al., Dopamine D2 receptor levels in striatum, thalamus, substantia nigra, limbic regions, and cortex in schizophrenic subjects. Biological Psychiatry, 2009. 65(12): p. 1024-31.

93. Suhara, T., et al., Decreased dopamine D2 receptor binding in the anterior cingulate cortex in schizophrenia. Archives of General Psychiatry, 2002. 59(1): p. 25-30.

94. Tuppurainen, $\mathrm{H}_{\text {., et al., Extrastriatal dopamine } D} 2 / 3$ receptor density and distribution in drug-naive schizophrenic patients. Molecular Psychiatry, 2003. 8(4): p. 453-5.

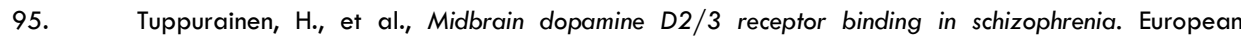
Archives of Psychiatry and Clinical Neuroscience, 2006. 256(6): p. 382-7.

96. Glenthoj, B.Y., et al., Frontal dopamine $D(2 / 3)$ receptor binding in drug-naive first-episode schizophrenic patients correlates with positive psychotic symptoms and gender. Biological Psychiatry, 2006. 60(6): p. $621-9$.

97. Arakawa, R., et al., Increase in thalamic binding of [(1 1)C]PE2I in patients with schizophrenia: a positron emission tomography study of dopamine transporter. Journal of Psychiatry Research, 2009. 43(15): p. 1219-23.

98. Cropley, V.L., et al., Pre- and post-synaptic dopamine imaging and its relation with frontostriatal cognitive function in Parkinson disease: PET studies with [1 1C]NNC 112 and [18F]FDOPA. Psychiatry Research, 2008. 163(2): p. 171-82.

99. Hall, H., et al., Distribution of D1- and D2-dopamine receptors, and dopamine and its metabolites in the human brain. Neuropsychopharmacology, 1994. 11 (4): p. 245-56.

100. Karlsson, P., et al., PET study of $D(1)$ dopamine receptor binding in neuroleptic-naive patients with schizophrenia. American Journal of Psychiatry, 2002. 159(5): p. 761-7.

101. Hirvonen, J., et al., Brain dopamine $d 1$ receptors in twins discordant for schizophrenia. American Journal of Psychiatry, 2006. 163(10): p. 1747-53.

102. Abi-Dargham, A., et al., Increased prefrontal cortical D1 receptors in drug-naive patients with schizophrenia: a PET study with [1 1C]NNC 11 2. Journal of Psychopharmacology, in press.

103. Guo, N., et al., Dopamine depletion and in vivo binding of PET D1 receptor radioligands: implications for imaging studies in schizophrenia. Neuropsychopharmacology, 2003. 28(9): p. 1703-11.

104. Slifstein, M., et al., [1 1C]NNC 112 selectivity for dopamine D1 and serotonin 5-HT(2A) receptors: a PET study in healthy human subjects. Journal of Cerebral Blood Flow and Metabolism, 2007. 27(10): p. 1733-41.

105. Ekelund, J., et al., In vivo DA D(1) receptor selectivity of NNC 112 and SCH 23390. Molecular Imaging and Biology, 2007. 9(3): p. 117-25.

106. Huttunen, J., et al., Striatal dopamine synthesis in first-degree relatives of patients with schizophrenia. Biological Psychiatry, 2008. 63(1): p. $114-7$.

107. Howes, O.D., et al., Progressive increase in striatal dopamine synthesis capacity as patients develop psychosis: a PET study. Molecular Psychiatry, 2011.

108. Soliman, A., et al., Stress-Induced Dopamine Release in Humans at Risk of Psychosis: a [(1 1)C]Raclopride PET Study. Neuropsychopharmacology, 2007.

109. Abi-Dargham, A., et al., Striatal amphetamine-induced dopamine release in patients with schizotypal personality disorder studied with single photon emission computed tomography and [123I]iodobenzamide. Biological Psychiatry, 2004. 55(10): p. 1001-6.

110. Woodward, N.D., et al., Correlation of Individual Differences in Schizotypal Personality Traits With Amphetamine-Induced Dopamine Release in Striatal and Extrastriatal Brain Regions. American Journal of Psychiatry, 2010. 
111. Hirvonen, J., et al., Increased caudate dopamine D2 receptor availability as a genetic marker for schizophrenia. Archives of General Psychiatry, 2005. 62(4): p. 371-8.

112. Lieberman, J.A., Neurobiology and the natural history of schizophrenia. J Clin Psychiatry, 2006. 67(10): p. el 14 .

113. American Psychiatric Association, Diagnostic and statistical manual of mental disorders. 4 ed. 2000, Washington, D.C.: Author.

114. Arnsten, A.F., Catecholamine and second messenger influences on prefrontal cortical networks of "representational knowledge": a rational bridge between genetics and the symptoms of mental illness. Cerebral Cortex, 2007. 17 Suppl 1: p. i6-15.

115. Goldman-Rakic, P.S. and L.D. Selemon, Functional and anatomical aspects of prefrontal pathology in schizophrenia. Schizophrenia Bulletin, 1997. 23(3): p. 437-58.

116. Arnsten, A.F., Prefrontal cortical network connections: key site of vulnerability in stress and schizophrenia. Int J Dev Neurosci, 2011.

117. Simpson, E.H., C. Kellendonk, and E. Kandel, A possible role for the striatum in the pathogenesis of the cognitive symptoms of schizophrenia. Neuron, 2010. 65(5): p. 585-96.

118. Kapur, S., R. Mizrahi, and M. Li, From dopamine to salience to psychosis--linking biology, pharmacology and phenomenology of psychosis. Schizophrenia Research, 2005. 79(1): p. 59-68.

$119 . \quad F l o r e s c o$, S.B., et al., Afferent modulation of dopamine neuron firing differentially regulates tonic and phasic dopamine transmission. Nat Neurosci, 2003. 6(9): p. 968-73.

120. Berridge, K.C. and T.E. Robinson, What is the role of dopamine in reward: hedonic impact, reward learning, or incentive salience? Brain Res Brain Res Rev, 1998. 28(3): p. 309-69.

121. Schultz, W., Predictive reward signal of dopamine neurons. J Neurophysiol, 1998. 80(1): p. 1-27.

122. Stuber, G.D., et al., Reward-predictive cues enhance excitatory synaptic strength onto midbrain dopamine neurons. Science, 2008. 321(5896): p. 1690-2.

123. Bilder, R.M., et al., The catechol-O-methyltransferase polymorphism: relations to the tonic-phasic dopamine hypothesis and neuropsychiatric phenotypes. Neuropsychopharmacology, 2004. 29(1 1): p. 1943-61.

124. Grace, A.A., Phasic versus tonic dopamine release and the modulation of dopamine system responsivity: a hypothesis for the etiology of schizophrenia. Neuroscience, 1991. 41(1): p. 1-24.

125. Grace, A.A., The tonic/phasic model of dopamine system regulation: its relevance for understanding how stimulant abuse can alter basal ganglia function. Drug Alcohol Depend, 1995. 37(2): p. 111 -29.

126. Smith, A., et al., Dopamine, prediction error and associative learning: a model-based account. Network, 2006. 17(1): p. 61-84.

127. Pessiglione, M., et al., Dopamine-dependent prediction errors underpin reward-seeking behaviour in humans. Nature, 2006. 442(7106): p. 1042-5.

128. Abler, B., et al., Prediction error as a linear function of reward probability is coded in human nucleus accumbens. Neuroimage, 2006. 31 (2): p. 790-5.

129. D'Ardenne, K., et al., BOLD responses reflecting dopaminergic signals in the human ventral tegmental area. Science, 2008. 319(5867): p. 1264-7.

130. Bayer, H.M. and P.W. Glimcher, Midbrain dopamine neurons encode a quantitative reward prediction error signal. Neuron, 2005. 47(1): p. 129-41.

131. Juckel, G., et al., Dysfunction of ventral striatal reward prediction in schizophrenia. Neuroimage, 2006. 29(2): p. 409-16.

132. Jensen, J., et al., The formation of abnormal associations in schizophrenia: neural and behavioral evidence. Neuropsychopharmacology, 2008. 33(3): p. 473-9.

133. Goldman-Rakic, P.S., The "psychic" neuron of the cerebral cortex. Ann N Y Acad Sci, 1999. 868: p. 13-26.

134. Lynch, M.R., Schizophrenia and the $D 1$ receptor: focus on negative symptoms. Prog Neuropsychopharmacol Biol Psychiatry, 1992. 16(6): p. 797-832. 
135. Goldman-Rakic, P.S., et al., Targeting the dopamine D1 receptor in schizophrenia: insights for cognitive dysfunction. Psychopharmacology (Berl), 2004. 174(1): p. 3-16.

136. Akil, M., et al., Decreased density of tyrosine hydroxylase-immunoreactive axons in the entorhinal cortex of schizophrenic subjects. Biologial Psychiatry, 2000. 47(5): p. 361-70.

137. Kellendonk, C., et al., Transient and selective overexpression of dopamine D2 receptors in the striatum causes persistent abnormalities in prefrontal cortex functioning. Neuron, 2006. 49(4): p. 603-15.

138. Drew, M.R., et al., Transient overexpression of striatal D2 receptors impairs operant motivation and interval timing. Journal of Neuroscience, 2007. 27(29): p. 7731-9.

139. Ward, R.D., et al., Impaired timing precision produced by striatal D2 receptor overexpression is mediated by cognitive and motivational deficits. Behav Neurosci, 2009. 123(4): p. 720-30.

140. Fusar-Poli, P., et al., Abnormal prefrontal activation directly related to pre-synaptic striatal dopamine dysfunction in people at clinical high risk for psychosis. Mol Psychiatry, 201 1. 16(1): p. 67-75.

141. Fusar-Poli, P., et al., Abnormal frontostriatal interactions in people with prodromal signs of psychosis: a multimodal imaging study. Arch Gen Psychiatry, 2010. 67(7): p. 683-91.

142. Deutch, A.Y., The regulation of subcortical dopamine systems by the prefrontal cortex: interactions of central dopamine systems and the pathogenesis of schizophrenia. J Neural Transm Suppl, 1992. 36: p. 61-89.

143. Meyer-Lindenberg, A., et al., Regionally specific disturbance of dorsolateral prefrontal-hippocampal functional connectivity in schizophrenia. Archives of General Psychiatry, 2005. 62(4): p. 379-86.

144. Harrison, P.J., The hippocampus in schizophrenia: a review of the neuropathological evidence and its pathophysiological implications. Psychopharmacology (Berl), 2004. 174(1): p. 151-62.

145. Lodge, D.J. and A.A. Grace, Hippocampal dysfunction and disruption of dopamine system regulation in an animal model of schizophrenia. Neurotoxicity Research, 2008. 14(2-3): p. 97-104. 



\title{
DOES DOPAMINE MEDIATE THE PSYCHOSIS-INDUCING EFFECTS OF CANNABIS? A REVIEW AND INTEGRATION OF FINDINGS ACROSS DISCIPLINES
}

\author{
Rebecca Kuepper ${ }^{1}$, Paul D. Morrison ${ }^{3}$, Jim van Os'1,3, Robin M. Murray3, 4,
} Gunter Kenis ${ }^{1} \&$ Cécile Henquet ${ }^{1,2}$

\footnotetext{
' Department of Psychiatry and Psychology, EURON, Maastricht University Medical Centre, South Limburg Mental Health Research and Teaching Network, Maastricht, The Netherlands. 2 Mondriaan Zorggroep, Division Addiction Care, South Limburg, The Netherlands 3 Psychosis Department, Institute of Psychiatry, Kings College, London, United Kingdom
} ${ }^{4}$ NIHR Biomedical Research Centre, Institute of Psychiatry, Kings College, London, United Kingdom

Schizophrenia Research, 2010. 121 (1-3): p. 107-117. 


\begin{abstract}
General population epidemiological studies have consistently found that cannabis use increases the risk of developing psychotic disorders in a dose-dependent manner. While the epidemiological signal between cannabis and psychosis has gained considerable attention, the biological mechanism whereby cannabis increases risk for psychosis remains poorly understood. Animal research suggests that delta-9tetrahydrocannabinol (THC, the main psychoactive component of cannabis) increases dopamine levels in several regions of the brain, including striatal and prefrontal areas. Since dopamine is hypothesized to represent a crucial common final pathway between brain biology and actual experience of psychosis, a focus on dopamine may initially be productive in the examination of the psychotomimetic effects of cannabis. Therefore, this review examines the evidence concerning the interactions between THC, endocannabinoids and dopamine in the cortical as well as subcortical regions implicated in psychosis, and considers possible mechanisms whereby cannabis-induced dopamine dysregulation may give rise to delusions and hallucinations. It is concluded that further study of the mechanisms underlying the link between cannabis and psychosis may be conducted productively from the perspective of progressive developmental sensitization, resulting from geneenvironment interactions.
\end{abstract}




\section{Introduction}

Stimulant drugs such as amphetamine have long been associated with psychotic symptoms. High doses can induce positive symptoms in people with no previous psychiatric history while even low doses can exacerbate positive symptoms in patients with a psychotic disorder [1, 2]. By the mid-1970s, experimental replications of the psychotomimetic effects of stimulant drugs, in combination with the observed antipsychotic effects of D2-blocking drugs, had given rise to the initial dopamine hypothesis of schizophrenia. Refined and modified in subsequent years, this theory remains central to theories about psychosis [3-6].

Recent developments suggest that the dopamine hypothesis may need to be reexamined in order to incorporate recent, seemingly unrelated research findings concerning risk factors for the development of psychosis [7]. One example concerns the insight that use of cannabis may impact negatively on mental health [8]. Cannabis use is associated with poor outcome in existing psychotic illness [9] and has consistently been shown to increase the risk to develop psychotic symptoms or disorder in healthy individuals [10-12]. However, the vast majority of cannabis users never develop any psychotic symptoms or mental health problems. Thus, cannabis is not a sufficient cause for psychotic illness but rather may constitute a component cause, interacting with other environmental and genetic factors [13]. Although the epidemiological link between cannabis and psychosis has been investigated extensively, the biological basis of this association remains poorly understood (see box 1 for a summary of main findings). Recent research suggests that heavy longterm cannabis use, particularly when started during adolescence, may lead to abnormalities in brain structure [14-18], but it seems unlikely that the use of cannabis increases the risk of psychosis by inducing major structural brain changes $[19,20]$. Its neurochemical interactions with neurotransmitters such as dopamine, however, are in urgent need of further investigation (DeLisi, 2008). Pharmacological and geneknockout studies have demonstrated that the central effects of THC are mediated via partial agonism at cannabinoid 1 receptors ( $C B 1$ receptors), the primary binding site of endogenous cannabinoids (eCBs) [21]. Expression of $C B 1$ receptors is high in the hippocampus, cerebellum, basal ganglia, prefrontal cortex (PFC), amygdala and substantia nigra pars reticulata [22]. Endocannabinoids, of which the best characterized are anandamide ( $\mathrm{N}$-arachidonoylethanolamine; AEA) and 2arachidonoylglycerol (2-AG), are synthesized in and released from postsynaptic neurons but act predominantly at $C B 1$ receptors located on neighboring presynaptic terminals, that is, they act in a retrograde fashion [23-25]. Activation of $C B 1$ receptors inhibits pre-synaptic neurotransmitter release (direct targets are GABA and glutamate terminals) and consequently modulates several neurotransmitter systems, including the dopamine system [26]. Furthermore, it is thought that principal 
output neurons, such as mesencephalic dopamine neurons, regulate their excitatory and inhibitory inputs via retrograde $\mathrm{eCB}$ signaling [26].

Box 1. What do we know about the association between cannabis and psychosis?

1. Epidemiological studies have shown that cannabis use increases the risk of developing psychotic symptoms in a dose-response fashion, independent of possible confounding factors and self-medication effects. Effects may be more prominent during early adolescence [1 1, 28-30].

2. Cannabis use is neither a necessary nor a sufficient cause of psychotic illness. Therefore, the link between cannabis use and psychosis may best be understood in terms of geneenvironment interactions [13]:

a. Patients with a diagnosis of schizophrenia are more sensitive to the cognitive and behavioral effects of cannabis than controls [31].

b. High schizotypy, psychotic disorder and COMT val158met genotype have been found to moderate sensitivity to the psychosis-inducing effects of cannabis [31-35].

c. Environmental risk factors may induce differential sensitivity to cannabis in terms of psychosis risk, e.g. childhood trauma $[36,37]$ and urban rearing environment [38].

3. Cumulative exposure to environmental risks may increase the risk for psychosis in an additive fashion. The concept of behavioral sensitization may therefore provide a plausible mechanism for the link between cannabis and psychosis [27, 39, 40].

4. Although some studies did not find cannabis-use related changes in brain morphology [41], others do suggest that long-term heavy cannabis use may lead to structural brain changes $[14,17,18]$, possibly related to early onset of use $[15,16]$.

5. Alterations in the endocannabinoid system have been revealed in schizophrenia, in particular increased levels of anandamide and increased $C B 1$ receptor availability, independent of cannabis use [42-44].

In order to discuss how modulation of dopaminergic neurotransmission by cannabinoids, including THC, may contribute to the development of psychotic symptoms - and ultimately schizophrenia - following persistent cannabis use, the literature (using the databases Pubmed and Psychinfo) was searched systematically for relevant experimental, clinical and epidemiological findings across different disciplines, including molecular, neurobiological and behavioral findings. This review will therefore first outline the involvement of dopamine in the pathophysiology of schizophrenia, with an emphasis on mechanisms of how dopaminergic dysregulations may translate into delusions and hallucinations. Subsequently, the question of how cannabinoids, such as THC, may impact on dopaminergic pathways to provoke psychosis will be examined. We also address the potential role of cannabinoids in sensitization [27] processes in psychosis. 


\section{What are the dopaminergic pathways to psychosis?}

The involvement of dopamine in the pathophysiology of schizophrenia is still central to the understanding of psychosis [45-47]. The classic dopamine hypothesis of schizophrenia assumed a hyperfunctioning dopamine system to be the central feature of schizophrenia symptomatology, primarily in terms of the positive symptom cluster [48, 49]. Additional formulations later proposed that reduced dopaminergic activity in prefrontal cortical brain regions might be linked to negative and cognitive symptoms [50-52]. Although current models of schizophrenia pathophysiology also consider other neurotransmitters such as glutamate and GABA $[4,46]$, dopamine is still assumed to play a key role in the emergence and experience of positive as well as negative and cognitive symptoms in schizophrenia $[6,53]$.

\section{The subcortical pathway}

Indirect evidence supporting the notion of a hyperfunction in the mesolimbic dopamine system comes from observations that amphetamine, through a mechanism of hyperdopaminergia, induces positive psychotic symptoms whereas antipsychotic medications, blocking dopamine D2 receptors, dampen symptoms [48, 49, 54, 55]. Direct empirical support for hyperdopaminergia, showing alterations in the mesolimbic dopamine system of schizophrenia patients, was initially elusive. While some studies found increased striatal D2 receptor availability in schizophrenia patients compared to healthy controls $[54,56,57]$, others failed to reveal such a difference [58-60]. However, more recent evidence suggests that it is elevated presynaptic dopamine function that is associated with schizophrenia [reviewed in 53].Furthermore, elevated pre-synaptic dopamine function was also reported in individuals with prodromal signs of schizophrenia [61] and in first-degree relatives of patients with schizophrenia [62].

Assuming that mesolimbic hyperdopaminergia plays an important role in the emergence and experience of psychotic symptoms, the question arises how dopaminergic alterations in mesolimbic brain regions actually give rise to the experience of hallucinations and delusions. Altered salience attribution has been suggested as a possible mechanism [63]. The mesolimbic dopamine system projects from the ventral tegmental area (VTA) to, among other areas, the ventral striatum. Burst firing of dopamine neurons in the VTA markedly increases dopamine release in the striatum [64] and is believed to mediate the perception of salience or reward associated with stimuli [65-67]. The phasic bursts of dopamine release, which are highly dependent on glutamatergic excitatory afferents, have been shown to be regulated by constant low-frequency tonic firing of dopamine neurons [68]. Tonic dopamine tone in turn is under control of GABAergic inhibition. Increased levels of tonic dopamine firing may result in decreased amplitude of phasic dopamine burst 
firing, thus dampening responsivity of this system. Decreased tonic dopamine levels, on the other hand, may result in a heightened responsivity of the phasic dopamine component [68, 69]. Kapur has suggested that, in schizophrenia, dopamine dysregulation results in a psychological state of aberrant salience, in which mundane events and ideas may be attributed with undue significance [63]. Thus, a hyperdopaminergic state in striatal brain regions is believed to create a condition in which logically unconnected ideas and associations are weaved together and elaborated upon, eventually leading to the emergence of a delusional system. Only a few experimental studies have actually studied the process of salience attribution in schizophrenia. This process has been related to associative and reinforcement learning, in which what is called "reward prediction error" plays a key role [70]. It is hypothesized that previous reward outcomes are used to form a reward prediction, which is then compared to the actual current reward. The difference between reward prediction and actual outcome is referred to as the reward prediction error [70]. Reward prediction error signaling is mediated, in animals as well as in humans, by dopamine activity in ventral midbrain and striatum [71-74]. Compared to healthy controls, patients with psychosis seem to exhibit aberrant reward prediction and reward-related learning, both at the behavioral and the neural level $[75,76]$. G. K. Murray and colleagues [77] employed a functional magnetic resonance imaging (fMRI) paradigm using a monetary reward task, and found that patients were less able to distinguish between neutral and rewarding stimuli. This was also evident at the brain activation level as patients with schizophrenia showed an increased midbrain response to neutral prediction, but attenuated activity in response to reward prediction [77]. Taken together, these results support the notion that hyperactivity in the mesolimbic dopamine system promotes psychotic symptoms by disrupting the process of salience attribution.

\section{The cortical pathway}

In addition to mesolimbic dopamine hyperfunction, schizophrenia has been associated with decreased activity in the mesocortical dopamine pathway, which projects from the VTA to, among other areas, the PFC [1]. As dopaminergic neurotransmission at prefrontal dopamine DI receptors is critical for PFC functioning and cognition [78, 79], cortical hypodopaminergia has been associated with the cognitive and negative symptoms of schizophrenia, such as impairments in working memory and executive functioning as well as anhedonia and flattened affect [50, 80, 81]. Evidence for this, however, has been mainly indirect and primarily stems from neuroimaging studies investigating prefrontal D1 receptor availability $[82,83]$.

\section{Subcortical and cortical dopamine: two distinct pathways?}

As outlined above, schizophrenia may be associated with dopamine imbalances in both cortical and subcortical brain regions. Furthermore, the mesocortical dopamine 
pathway, projecting from VTA to PFC, has been shown to exert inhibitory influences on mesolimbic dopamine activity $[84,85]$. It has thus been proposed that mesolimbic hyperdopaminergia in schizophrenia may result from failed inhibition by cortical dopamine [84, 85]. Meyer-Lindenberg and colleagues [86] used Positron Emission Tomography to study regional cerebral blood flow (with $\left[{ }^{15} \mathrm{O}\right] \mathrm{H}_{2} \mathrm{O}$ as radiotracer) and presynaptic dopaminergic function (with $6-[18 \mathrm{~F}] \mathrm{DOPA}$ as radiotracer) in the same session. Patients with a diagnosis of schizophrenia exhibited significantly lower levels of prefrontal cortical activity during an executive functioning task, compared to controls. Furthermore, the decrease in PFC activity in the patient group was predictive of a relative increase in striatal dopaminergic function, indicating that frontal hypodopaminergia leads to mesolimbic hyperdopaminergia.

While the above suggests that striatal dopamine transmission is under the influence of PFC dopamine, Kellendonk and colleagues have demonstrated the opposite as well: mesolimbic dopamine activity may exert influences on prefrontal dopamine function [87-89]. Transgenic mice over-expressing striatal D2 receptors were found to be characterized by impairments in working memory and executive functioning, both of which are functions that critically require prefrontal dopamine activity. At the molecular level, over-expression of striatal D2 receptors was associated with increased dopamine turnover in the PFC and increased activation of prefrontal DI receptors [87]. It was further demonstrated that over-expression of striatal D2 receptors resulted in disturbed motivational and associative learning [88, 89], core features of schizophrenia. There is thus some evidence that the cortical and subcortical dopamine pathways are subject to bi-directional influences.

\section{Does THC influence dopaminergic pathways to psychosis?}

Is it possible that some of the psychosis-inducing effects of exogenous cannabinoids such as THC may be mediated by dopamine? THC has been shown to affect endocannabinoid neurotransmission and much evidence indicates that eCBs are key components in the regulation of dopamine neurotransmission [90-92]. Animal research furthermore suggests that exogenous cannabinoids like THC facilitate dopaminergic neurotransmission in several regions of the brain, including striatum and PFC [93-95].

\section{Do cannabinoids influence subcortical dopamine?}

Dopamine neuronal firing in the VTA is controlled by excitatory glutamatergic and inhibitory GABAergic inputs, which in turn are reciprocally modulated by eCBs released from dopaminergic dendrites (figure 1) [91, 96, 97]. Findings from numerous animal studies have shown that the overall result of exogenous $C B 1$ 
receptor agonists, such as THC, is to evoke burst-firing in the VTA and thereby increase extracellular dopamine concentrations in striatal brain regions (figure 1) [94, 98-100]. Recently, the acute effects of THC on striatal dopamine transmission have been investigated in human volunteers, using experimental Positron Emission Tomography (PET) paradigms. However, findings were inconsistent. While one study reported a THC-induced increase in striatal dopamine release [101], another study failed to detect such an effect [102]. An earlier Single Photon Emission Computed Tomography (SPECT) study, however, did find increased dopamine release in the striatum of a single patient with schizophrenia following cannabis exposure; in addition, the dopamine response in the patient was followed by an acute worsening of positive psychotic symptoms [103]. Researchers have therefore asked whether D2 blockade might prevent such an effect. D'Souza and colleagues showed that haloperidol (a D2 antagonist) did not significantly inhibit acute THC induced psychopathology in healthy subjects, which was in agreement with their earlier finding that ongoing D2 blockade in schizophrenia patients (i.e. antipsychotic treatment) offered no protection against THC-elicited acute positive psychotic symptoms [104, 105]. Liem-Moolenaar [106] on the other hand demonstrated that pre-treatment with haloperidol did reverse THC-induced increases in Positive and Negative Syndrome Scale scores in healthy volunteers [106].

At the molecular level, both dopamine and $\mathrm{eCBs}$ are fundamental for the synaptic re-organisation which underpins new striatal learning [107-110]. So called spiketiming dependent plasticity (STDP) has an absolute requirement for nearsimultaneous electrical activity in pre-synaptic terminals (cortical inputs) and the dendritic spines of medium spiny neurons (MSNs), the major striatal neuron. Crucially, change occurs rapidly, after only one or two spike pairings, conditions which are feasible under normal physiological conditions [ 1111,112$]$. In the striatum, long-term depression (LTD) of cortical inputs onto MSNs has repeatedly been shown to be mediated by retrograde endocannabinoid signaling [113-115]. In addition corticostriatal LTD often requires the activation of D2 receptors by dopamine (figure 2) $[111,116,117]$. For instance, in mice in which dopamine was depleted by reserpine or 6-hydroxy-dopamine, LTD of corticostriatal synapses was absent but could be restored by administration of the D2 receptor agonist quinpirole; and quinpirole combined with an inhibitor of $\mathrm{eCB}$ clearance showed synergistic effects in improving the severe psychomotor impairments associated with dopamine depletion [1 18 ]. 
A

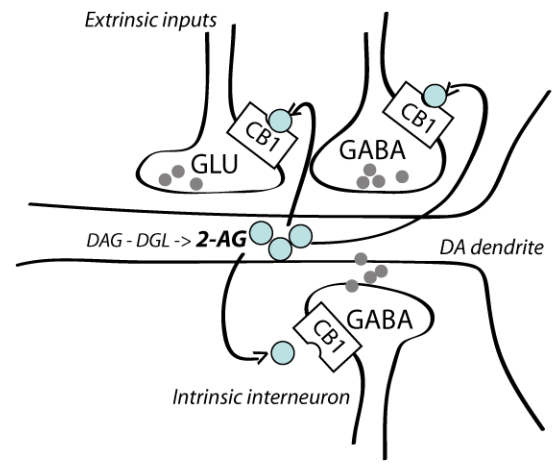

B

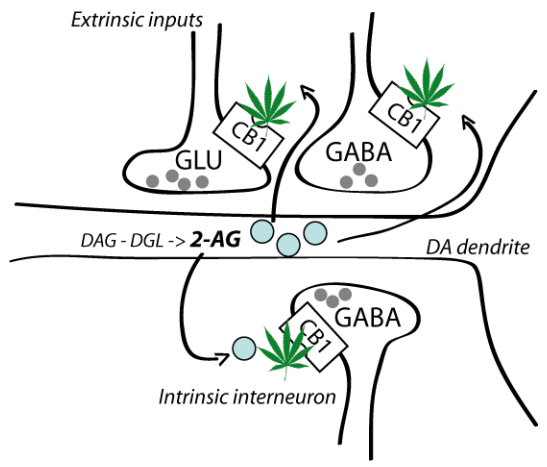

Figure 1. The convergence of dopamine and the endocannabinoids in the VTA.

A. Firing patterns in midbrain dopaminergic (DA) neurons are influenced by a host of excitatory (glutamatergic, GLU, as indicated in grey) and inhibitory (GABAergic, GABA, as indicated in grey) inputs. DA neurons regulate neighboring presynaptic terminals via retrograde endocannabinoid (2-AG, as indicated in blue) signaling.

B. When exogenous cannabinoids (THC, as indicated by cannabis leaves) bind to $C B 1$ R's located on glutamatergic (GLU) and GABAergic (GABA) terminals, retrograde endocannabinoid signaling (2-AG, as indicated in blue) is disrupted and stimulation of $C B 1$ R's by THC inhibits glutamate and GABA release.

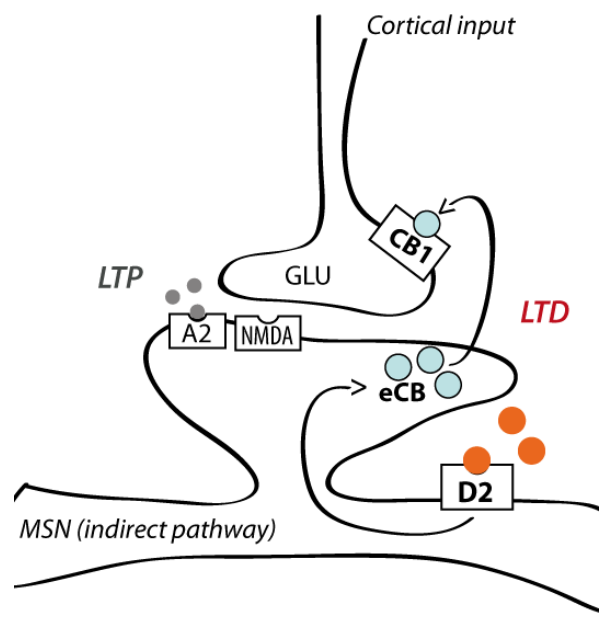

Figure 2. Striatal plasticity at medium spiny neurons belonging to the indirect pathway. Cortical fibers form glutamatergic synapses (GLU) at the medium spiny neuron (MSN). At these synapses, endocannabinoidmediated (as indicated in blue) long-term depression (LTD) is induced as long as dopamine (as indicated in orange) is present at D2 receptors. In the absence of dopamine or in the presence of $A_{2 A}$ receptor agonists (as indicated in grey) long-term potentiation (LTP) is induced. 
New insights into the roles of dopamine and $\mathrm{eCBs}$ in striatal learning and plasticity have been provided by Shen and colleagues [112]. They investigated plasticity at identified populations of striatal MSNs. MSNs can be divided into two roughly equally sized groups. MSNs belonging to the direct pathway express DI receptors while MSNs of the indirect pathway express D2 receptors. Shen et al. [1 112 ] showed that cortical inputs onto both MSN sub-types exhibit bi-directional plasticity (strengthening or weakening), dependent on the pattern of ongoing neuromodulation: At D2 MSNs, LTD was expressed pre-synaptically via retrograde endocannabinoid signaling, but only as long as dopamine was present at D2 receptors. Co-joint adenosine $A_{2 a}$ receptor and glutamate NMDA receptor stimulation could overpower LTD and trigger LTP instead (figure 2). For completeness, the opposite was observed at Dl expressing MSNs. Here, endocannabinoid-mediated LTD was induced in the absence of dopamine from DI receptors, whereas D1 agonists led to the induction of LTP [112]. These findings indicate that eCBs, glutamate, adenosine and dopamine act in concert to promote plasticity at simultaneously active corticostriatal synapses. One could speculate that under tonic D2 receptor stimulation, active MSN's belonging to the indirect pathway weaken their cortical inputs via endocannabinoid-mediated LTD (figure 2) and a net positive signal would be returned to the cortex, facilitating an emerging psychomotor program. In contrast, if extracellular dopamine levels fall, albeit transiently following the non-arrival of a predicted reward [119], the indirect pathway would tend towards LTP (strengthening), and an overall negative signal would be returned to the cortex. Failure to signal the non-arrival of reward, that is, failure to inhibit tonic D2 signaling would be predicted to have major effects on corticostriatal loops and psychomotor health.

Returning to the pharmacology, caffeine, an antagonist at $A_{2 A}$ receptors (LTP blockade), has been shown to exacerbate positive psychotic symptoms as well [120, 121], while $A_{2 A}$ receptor agonists (LTP promotion) such as CGS 21680 appear to have anti-psychotic properties [122, 123]. Further, inhibitors of adenosine re-uptake or metabolic degradation have anti-psychotic properties in schizophrenia patients [124-127]. In addition, it is widely acknowledged that CB 1 receptor agonists, such as THC (LTD promotion), are psychotomimetic and constitute a component risk factor for the development of schizophrenia, whereas cannabidiol (CBD) an allosteric inverse agonist at $C B 1$ receptors (and inhibitor of adenosine re-uptake) has shown promise as an antipsychotic [128-131]. Overall, pharmaceutical manipulations which alter the balance of corticostriatal synapses of the indirect pathway display consistent pro- or anti-psychotic properties. Drugs which weaken cortical inputs onto MSNs of the indirect pathway (LTD) appear to be pro-psychotic. In contrast, drugs which favor a shift towards strengthening (LTP) of the same synapses appear to be anti-psychotic [132]. 


\section{Do cannabinoids interact with the cortical dopamine pathway?}

In addition to influencing dopaminergic neurotransmission in the mesolimbic pathway, THC also seems to affect dopaminergic neurotransmission in the PFC, at least as far as evidence from animal studies is concerned [93, 133]. At the human behavioral level, the acute effects of THC include cognitive impairment in domains such as memory and attention [134, 135]. Furthermore, short term cognitive impairment following cannabis use has been suggested to overlap largely with cognitive dysfunctions as observed in schizophrenia [136]. It is well established that cognitive performance strongly depends on optimal prefrontal dopamine levels, with too low as well as too high levels of dopamine being equally detrimental to cognition [78]. In rats, acute administration of THC has been shown to increase prefrontal dopamine levels [137, 138], and to impair spatial working memory [139]. Furthermore, Pistis and colleagues [138] found that THC-induced increases in prefrontal dopamine could be prevented by administration of rimonabant, a CB1 receptor antagonist. This suggests that the effects of THC on dopamine transmission are mediated by the activation of $C B 1$ receptors [138]. In contrast to these acute effects, repeated exposure to THC has been found to result in a reduction of dopamine metabolism in the rat PFC [133, 140]. The mechanism underlying the differences associated with acute and repeated exposure need to be investigated further - sensitization, as discussed below, may provide a concept to advance this field. The effects of repeated exposure again were accompanied by cognitive impairment, which follows the inverted U-curve association between dopamine levels and cognitive performance [141]. In an experimental study in human volunteers, carriers of the Val allele of the COMT (catechol-o-methyltransferase) vall 58met polymorphism who had repeatedly used cannabis were more sensitive to both the psychosis-inducing and the cognitive effects of THC than Met allele carriers [32]. The Val allele of this common polymorphism is associated with higher enzymatic activity of COMT and accordingly lower levels of prefrontal dopamine [142].

Although THC has been found to affect dopaminergic processes in PFC, the nature of endocannabinoid function in the human PFC remains largely unknown [143]. CB 1 receptors are densely expressed in the PFC [144] and studies on CB 1 receptor knock-out mice have provided insights in prefrontal endocannabinoid function. By using the Morris water maze paradigm, Varvel \& Lichtman [145] showed that CB 1 receptor knock-out mice did not differ from wild type control mice with regard to recognition memory. However, $C B 1$ receptor knock-out mice made significantly more perseveration errors after the location of the platform had been changed. In addition, administration of THC was found to significantly worsen mnemonic performance in the wild type control mice, while task performance of the $C B 1$ receptor knock-out was unaffected [145]. Since memory function partly relies on 
prefrontal brain functioning, it may be hypothesized that the observed performance impairments are attributable to THC effects in prefrontal brain regions.

Taken together, it seems that the repeated administration of THC alters PFC function and impairs cognition by acting on dopamine signaling via activation of $C B 1$ receptors. In particular, repeated exposure to THC has been found to decrease dopamine levels in the PFC. As decreased prefrontal dopaminergic function is postulated to constitute a core feature of schizophrenia pathophysiology [82], cannabis use may increase the risk for psychosis partly by contributing to a hypodopaminergic state in the PFC. Taking into account THC effects in striatal regions, it thus appears that THC aggravates dopaminergic imbalances by increasing the dopaminergic tone in striatal regions of the brain, while, when administered repeatedly, decreasing dopamine levels in prefrontal regions of the brain. Evidence to support this idea predominantly stems from animal research and it remains speculative whether this applies to humans as well.

\section{Does dopamine-mediated behavioral sensitization constitute a potential mechanism behind the cannabis-psychosis relationship?}

The above suggests that repeated exposure to THC may cause changes in the dopamine system and subsequently progressively greater behavioral responses over time [146, 147]. A process of dopamine sensitization thus may constitute a possible mechanism by which environmental factors such as stress or stimulant drugs contribute to psychosis risk (figure 3) [27, 148, 149]. Rats pretreated with THC twice a day over a period of three days, were found to be more sensitive to the locomotor effects of a THC challenge, after a 14-day washout period, compared to rats that had not been pretreated [146]. Neurobiologically, the process of sensitization is thought to involve dopaminergic signaling in the mesolimbic pathway, as sensitization to stimulants, such as amphetamine, has frequently been shown to lead to increased activity of dopamine neurons in the VTA [150] and increased dopamine release in the striatum [151-154] [147].

Cross-sensitization between stimulant drugs and stress has been shown as well. De Jong and colleagues [155] demonstrated that mice, after being exposed to psychosocial stress using a social defeat paradigm, exhibited an enhanced locomotor response to an amphetamine challenge compared to mice that had not been stressed previously. Using repeated maternal separation as a psychosocial stress paradigm, Kikusui and co-workers [156] found that mice which were kept separated from their mothers from day 1 to day 13 in the post-natal period, were more sensitive to the locomotor effects of a cocaine challenge at day 50, than mice which had not been stressed [156]. Most likely, cross-sensitization between stress and stimulants is mediated by striatal dopamine, as cocaine-induced striatal dopamine 
increase was higher in rats which had been repeatedly pre-exposed to a stressor in form of foot shocks $[157,158]$.

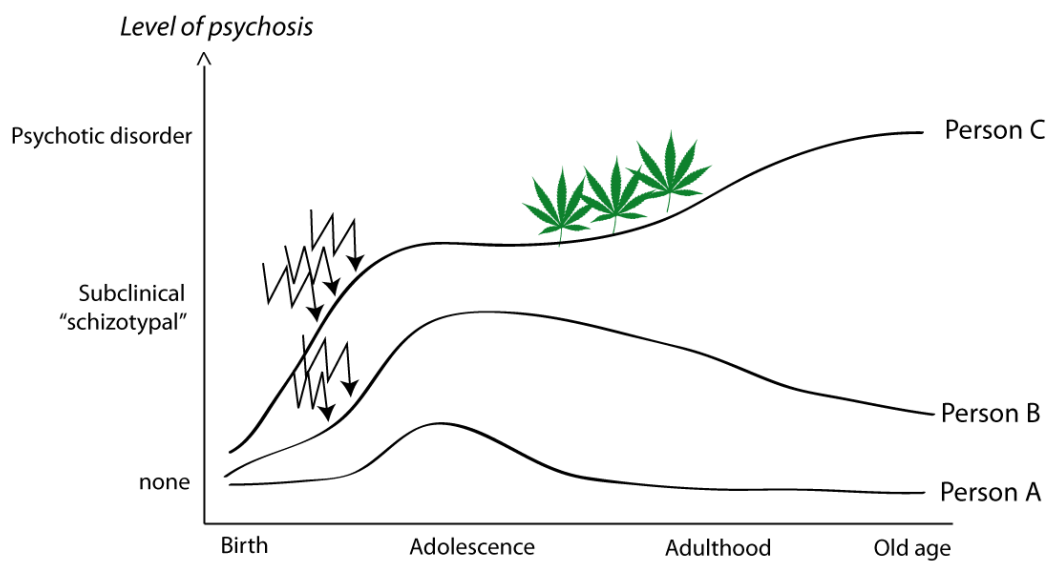

Figure 3. Sensitization Behavioral Phenotype. Person A has "normal" developmental expression of subclinical psychotic experiences that are mild and transient. Person B has similar expression but longer persistence due to additional environmental exposure such as stress (as indicated by zigzag arrows). Person $C$ has prolonged persistence and subsequent transition to clinical psychotic disorder possibly due to severe repeated environmental exposure such as repeated cannabis use (as indicated by cannabis leaves) in addition to prior stress. Adapted with permission from Collip et al., 2008.

Although THC shows cross-sensitization with other drugs such as amphetamine, heroin and morphine [146, 159], only a few studies have investigated cross-sensitization between THC and stress. Findings from animal studies have shown that the effects of THC seem to be contingent on the environmental conditions under which THC is administered [160]. In rats housed under normal conditions (housed in groups with water and food freely available) the exposure to THC only caused slight behavioral changes in form of mild sedation and hypothermia and did not alter dopaminergic neurotransmission. In contrast, under stressful housing conditions (isolation and food deprivation), THC administration had marked behavioral consequences, including immobility and hyperactivity. Furthermore, exposure to THC in the stressful environment resulted in significantly increased striatal dopamine uptake [160]. Mokler and colleagues [161] showed that in rats, pre-treatment with THC on days 4, 6 and 8 postnatally changed the behavioral response to electric foot shocks in the adult period. THC pretreatment furthermore altered the stress-induced dopamine response in the hypothalamus and frontal cortex [161]. Houston [36] investigated interaction between early cannabis use and childhood sexual trauma on the development of psychotic disorder in a large population-based sample ( $N=5877)$. 
The data were suggestive of cross-sensitization between stress and early cannabis exposure, as the effect of cannabis on psychosis outcome was significant only in the individuals who had been exposed to trauma during early childhood. The interactive effective of cannabis use and childhood trauma on psychosis risk was replicated by Harley et al. [37].

At the molecular level, sensitization processes seem to involve endocannabinoid signaling. Filip and colleagues [162] demonstrated that administration of rimonabant, a CB1 receptor antagonist potently blocked the expression of hypersensitivity to cocaine [162]. Further, Corbille and colleagues showed that sensitization following a single dose of cocaine or amphetamine was reduced in $C B 1$ receptor knockout mice [163] and $C B 1$ receptor knockouts failed to sensitize to amphetamine administered daily for 7 days [164]. In addition, it was shown that mice treated with the $C B 1$ receptor antagonist $A M 251$ in both a single and 7 day dosing paradigm showed a reduction in the development of sensitization to stimulants [165]. Finally, Chiang and Chen showed that microinjection of the CB1-R inverse agonist/antagonist SR 147778 directly into the ventral striatum potently blocked the expression of hypersensitivity to methamphetamine (Chiang and Chen, 2007).

Thus, despite some contradictory findings, research suggests an important role for the endocannabinoid system in the neurochemical processes underlying sensitization. Repeated exposure to THC may sensitize an individual to the psychotic effects of THC, in interaction with other environmental risk factors such as stress, and this may be particularly relevant for individuals genetically at risk of dopamine dysregulation (Henquet, 2008).

\section{Conclusion}

Although advances in the understanding of endocannabinoid function have been made, the endocannabinoid system is still far from being understood and its interactions with other neurotransmitter systems including the dopamine system are complex. While in the VTA, dopamine seems to be downstream of endocannabinoid function, it appears that, in the ventral striatum, part of dopaminergic neurotransmission is actually upstream of endocannabinoid function, at least concerning its involvement in plasticity at striatal MSN's. Accordingly, although THC might stimulate burst firing of DA neurons in the VTA and as a consequence increase DA levels in the striatum, here THC might additionally exert its effects by directly influencing synaptic plasticity [132]. 
Most evidence to date, however, stems from animal research and to date, a clear answer to the question of whether there is a case for dopamine mediating the psychosis-inducing effects of cannabis cannot be given. There is a lack of studies investigating the acute and long term effects of THC on dopaminergic neurotransmission, learning and reward experience in humans. Advances in imaging techniques, such as the development of novel radiotracers, as well as a growing understanding of the biology and psychology of positive psychotic symptoms, hold promise for the future study of how the biological underpinnings of the cannabispsychosis relationship may alter experience in such a way that psychotic symptoms ensue. 


\section{References}

1. Abi-Dargham, A., Do we still believe in the dopamine hypothesis? New data bring new evidence. International Journal of Neuropsychopharmacology, 2004. 7 Suppl 1: p. S1-5.

2. Curran, C., N. Byrappa, and A. McBride, Stimulant psychosis: systematic review. British Journal of Psychiatry, 2004. 185: p. 196-204.

3. Lieberman, J.A., B.B. Sheitman, and B.J. Kinon, Neurochemical sensitization in the pathophysiology of schizophrenia: deficits and dysfunction in neuronal regulation and plasticity. Neuropsychopharmacology, 1997. 17(4): p. 205-29.

4. Laruelle, M., L.S. Kegeles, and A. Abi-Dargham, Glutamate, dopamine, and schizophrenia: from pathophysiology to treatment. Ann N Y Acad Sci, 2003. 1003: p. 138-58.

5. Carlsson, A. and M.L. Carlsson, A dopaminergic deficit hypothesis of schizophrenia: the path to discovery. Dialogues Clin Neurosci, 2006. 8(1): p. 137-42.

6. Murray, R.M., J. Lappin, and M. Di Forti, Schizophrenia: from developmental deviance to dopamine dysregulation. European Neuropsychopharmacology, 2008. 18 Suppl 3: p. S129-34.

7. Di Forti, M., J.M. Lappin, and R.M. Murray, Risk factors for schizophrenia--all roads lead to dopamine. Eur Neuropsychopharmacol, 2007. 17 Suppl 2: p. S101-7.

8. Murray, R.M., et al., Cannabis, the mind and society: the hash realities. Nature Reviews. Neuroscience, 2007. 8(11): p. 885-895.

9. Zammit, S., et al., Effects of cannabis use on outcomes of psychotic disorders: systematic review. British Journal of Psychiatry, 2008. 193(5): p. 357-63.

10. Henquet, C., et al., The environment and schizophrenia: the role of cannabis use. Schizophrenia Bulletin, 2005. 31(3): p. 608-12.

11. Moore, T.H., et al., Cannabis use and risk of psychotic or affective mental health outcomes: a systematic review. Lancet, 2007. 370(9584): p. 319-28.

12. Semple, D.M., A.M. Mclntosh, and S.M. Lawrie, Cannabis as a risk factor for psychosis: systematic review. Journal of Psychopharmacology, 2005. 19(2): p. 187-94.

13. Henquet, C., et al., Gene-environment interplay between cannabis and psychosis. Schizophrenia Bulletin, 2008. 34(6): p. $1111-21$.

14. Yucel, M., et al., Regional brain abnormalities associated with long-term heavy cannabis use. Arch Gen Psychiatry, 2008. 65(6): p. 694-701.

15. Wilson, W., et al., Brain morphological changes and early marijuana use: a magnetic resonance and positron emission tomography study. J Addict Dis, 2000. 19(1): p. 1-22.

16. Arnone, D., et al., Corpus callosum damage in heavy marijuana use: preliminary evidence from diffusion tensor tractography and tract-based spatial statistics. Neuroimage, 2008. 41(3): p. 1067 . 74.

17. Matochik, J.A., et al., Altered brain tissue composition in heavy marijuana users. Drug Alcohol Depend, 2005. 77(1): p. 23-30.

18. Welch, K.A., et al., The Impact of Substance Use on Brain Structure in People at High Risk of Developing Schizophrenia. Schizophrenia Bulletin, 2010.

19. DeLisi, L.E., The effect of cannabis on the brain: can it cause brain anomalies that lead to increased risk for schizophrenia? Curr Opin Psychiatry, 2008. 21(2): p. 140-50.

20. Linszen, D. and T. van Amelsvoort, Cannabis and psychosis: an update on course and biological plausible mechanisms. Current Opinion in Psychiatry, 2007. 20(2): p. 116-20.

21. Pertwee, R.G., Cannabinoid pharmacology: the first 66 years. Br J Pharmacol, 2006. 147 Suppl 1: p. S163-71.

22. Freund, T.F., I. Katona, and D. Piomelli, Role of endogenous cannabinoids in synaptic signaling. Physiol Rev, 2003. 83(3): p. 1017-66. 
23. Wilson, R.I. and R.A. Nicoll, Endogenous cannabinoids mediate retrograde signalling at hippocampal synapses. Nature, 2001. 410(6828): p. 588-92.

24. Devane, W.A., et al., Isolation and structure of a brain constituent that binds to the cannabinoid receptor. Science, 1992. 258(5090): p. 1946-9.

25. Mechoulam, R., et al., Identification of an endogenous 2-monoglyceride, present in canine gut, that binds to cannabinoid receptors. Biochem Pharmacol, 1995. 50(1): p. 83-90.

26. Chevaleyre, V., K.A. Takahashi, and P.E. Castillo, Endocannabinoid-Mediated Synaptic Plasticity in the CNS. Annu Rev Neurosci, 2006.

27. Collip, D., I. Myin-Germeys, and J. Van Os, Does the concept of "sensitization" provide a plausible mechanism for the putative link between the environment and schizophrenia? Schizophrenia Bulletin, 2008. 34(2): p. 220-5.

28. Konings, M., et al., Early exposure to cannabis and risk for psychosis in young adolescents in Trinidad. Acta Psychiatr Scand, 2008.

29. Arseneault, L., et al., Cannabis use in adolescence and risk for adult psychosis: longitudinal prospective study. British Medical Journal, 2002. 325(7374): p. 1212-3.

30. McGrath, J., et al., Association Between Cannabis Use and Psychosis-Related Outcomes Using Sibling Pair Analysis in a Cohort of Young Adults. Arch Gen Psychiatry, 2010.

31. D'Souza, D.C., et al., Delta-9-tetrahydrocannabinol effects in schizophrenia: implications for cognition, psychosis, and addiction. Biological Psychiatry, 2005. 57(6): p. 594-608.

32. Henquet, C., et al., An experimental study of catechol-o-methyltransferase Val158Met moderation of delta-9-tetrahydrocannabinol-induced effects on psychosis and cognition. Neuropsychopharmacology, 2006. 31 (12): p. $2748-57$.

33. Henquet, C., et al., Prospective cohort study of cannabis use, predisposition for psychosis, and psychotic symptoms in young people. British Medical Journal, 2005. 330(7481): p. 11.

34. Caspi, A., et al., Moderation of the effect of adolescent-onset cannabis use on adult psychosis by a functional polymorphism in the catechol-O-methyltransferase gene: longitudinal evidence of a gene $X$ environment interaction. Biological Psychiatry, 2005. 57(10): p. 1117-27.

35. van Os, J., et al., Cannabis use and psychosis: a longitudinal population-based study. American Journal of Epidemiology, 2002. 156(4): p. 319-27.

36. Houston, J.E., et al., Childhood sexual abuse, early cannabis use, and psychosis: testing an interaction model based on the National Comorbidity Survey. Schizophrenia Bulletin, 2008. 34(3): p. 580-5.

37. Harley, M., et al., Cannabis use and childhood trauma interact additively to increase the risk of psychotic symptoms in adolescence. Psychol Med, 2009: p. 1-8.

38. Henquet, C., et al., Do cannabis and urbanicity interact in causing psychosis? Schizophrenia Bulletin, 2009. 35(supplement 1): p. 87.

39. Cougnard, A., et al., Does normal developmental expression of psychosis combine with environmental risk to cause persistence of psychosis? A psychosis proneness-persistence model. Psychological Medicine, 2007. 37(4): p. 513-27.

40. Dominguez, M.D., et al., Evidence That Onset of Clinical Psychosis Is an Outcome of Progressively More Persistent Subclinical Psychotic Experiences: An 8-Year Cohort Study. Schizophr Bull, 2009. Published Online May 21 2009; doi:10.1093/schbul/sbp022

41. Delisi, L.E., et al., A preliminary DTI study showing no brain structural change associated with adolescent cannabis use. Harm Reduct J, 2006. 3: p. 17.

42. Leweke, M., et al., Elevated endogenous cannabinoids in schizophrenia. Neuroreport, 1999. 10(8): p. 1665-9.

43. Dean, B., et al., Studies on [3H]CP-55940 binding in the human central nervous system: regional specific changes in density of cannabinoid-1 receptors associated with schizophrenia and cannabis use. Neuroscience, 2001. 103(1): p. 9-15.

44. Koethe, D., et al., Expression of CB1 cannabinoid receptor in the anterior cingulate cortex in schizophrenia, bipolar disorder, and major depression. J Neural Transm, 2007. 114(8): p. 1055-63. 
45. Carlsson, A., Does dopamine play a role in schizophrenia? Psychological Medicine, 1977. 7(4): p. 583-97.

46. Carlsson, A., The Neurochemical Circuitry of Schizophrenia. Pharmacopsychiatry, 2006. 39 (suppl.1): p. 10-14.

47. Guillin, O., A. Abi-Dargham, and M. Laruelle, Neurobiology of dopamine in schizophrenia. Int Rev Neurobiol, 2007. 78: p. 1-39.

48. Kapur, S. and D. Mamo, Half a century of antipsychotics and still a central role for dopamine D2 receptors. Prog Neuropsychopharmacol Biol Psychiatry, 2003. 27(7): p. 1081-90.

49. Carlsson, A., Antipsychotic drugs, neurotransmitters, and schizophrenia. American Journal of Psychiatry, 1978. 135(2): p. 165-73.

50. Lynch, M.R., Schizophrenia and the D1 receptor: focus on negative symptoms. Prog Neuropsychopharmacol Biol Psychiatry, 1992. 16(6): p. 797-832.

51. Weinberger, D.R., Implications of normal brain development for the pathogenesis of schizophrenia. Arch Gen Psychiatry, 1987. 44(7): p. 660-9.

52. Murray, R.M. and S.W. Lewis, Is schizophrenia a neurodevelopmental disorder? Br Med J (Clin Res Ed), 1987. 295(6600): p. 681-2.

53. Howes, O.D. and S. Kapur, The dopamine hypothesis of schizophrenia: version III--the final common pathway. Schizophrenia Bulletin, 2009. 35(3): p. 549-62.

54. Seeman, P., Dopamine receptors and the dopamine hypothesis of schizophrenia. Synapse, 1987. 1(2): p. 133-52.

55. Laruelle, M. and A. Abi-Dargham, Dopamine as the wind of the psychotic fire: New evidence from brain imaging studies. Journal of Psychopharmacology, 1999. 13: p. 358-371.

56. Abi-Dargham, A., et al., Increased baseline occupancy of D2 receptors by dopamine in schizophrenia. Proceedings of the National Acadademy of Science 2000. 97(14): p. 8104-9.

57. Hietala, J., et al., Striatal D2 dopamine receptor characteristics in neuroleptic-naive schizophrenic patients studied with positron emission tomography. Archives of General Psychiatry, 1994. 51(2): p. 116-23.

58. Martinot, J.L., et al., Striatal D2 dopaminergic receptors assessed with positron emission tomography and $[76 \mathrm{Br}]$ bromospiperone in untreated schizophrenic patients. Am J Psychiatry, 1990. 147(1): p. 4450 .

59. Nordstrom, A.L., et al., No elevated D2 dopamine receptors in neuroleptic-naive schizophrenic patients revealed by positron emission tomography and [1 1C]N-methylspiperone. Psychiatry Res, 1995. 61(2) p. $67-83$.

60. Pilowsky, L.S., et al., D2 dopamine receptor binding in the basal ganglia of antipsychotic-free schizophrenic patients. An 123I-IBZM single photon emission computerised tomography study. $\mathrm{Br} J$ Psychiatry, 1994. 164(1): p. 16-26.

61. Howes, O.D., et al., Elevated striatal dopamine function linked to prodromal signs of schizophrenia. Archives of General Psychiatry, 2009. 66(1): p. 13-20.

62. Huttunen, J., et al., Striatal dopamine synthesis in first-degree relatives of patients with schizophrenia. Biological Psychiatry, 2008. 63(1): p. 114-7.

63. Kapur, S., R. Mizrahi, and M. Li, From dopamine to salience to psychosis--linking biology, pharmacology and phenomenology of psychosis. Schizophrenia Research, 2005. 79(1): p. 59-68.

64. Floresco, S.B., et al., Afferent modulation of dopamine neuron firing differentially regulates tonic and phasic dopamine transmission. Nat Neurosci, 2003. 6(9): p. 968-73.

65. Berridge, K.C. and T.E. Robinson, What is the role of dopamine in reward: hedonic impact, reward learning, or incentive salience? Brain Res Brain Res Rev, 1998. 28(3): p. 309-69.

66. Schultz, W., Predictive reward signal of dopamine neurons. J Neurophysiol, 1998. 80(1): p. 1-27.

67. Stuber, G.D., et al., Reward-predictive cues enhance excitatory synaptic strength onto midbrain dopamine neurons. Science, 2008. 321(5896): p. 1690-2. 
68. Goto, Y., S. Otani, and A.A. Grace, The yin and yang of dopamine release: a new perspective. Neuropharmacology, 2007. 53: p. 583-587.

69. Bilder, R.M., et al., The catechol-O-methyltransferase polymorphism: relations to the tonic-phasic dopamine hypothesis and neuropsychiatric phenotypes. Neuropsychopharmacology, 2004. 29(11): p. 1943-61.

70. Smith, A., et al., Dopamine, prediction error and associative learning: a model-based account. Network, 2006. 17(1): p. 61-84.

71. Pessiglione, M., et al., Dopamine-dependent prediction errors underpin reward-seeking behaviour in humans. Nature, 2006. 442(7106): p. 1042-5.

72. Abler, B., et al., Prediction error as a linear function of reward probability is coded in human nucleus accumbens. Neuroimage, 2006. 31(2): p. 790-5.

73. D'Ardenne, K., et al., BOLD responses reflecting dopaminergic signals in the human ventral tegmental area. Science, 2008. 319(5867): p. 1264-7.

74. Bayer, H.M. and P.W. Glimcher, Midbrain dopamine neurons encode a quantitative reward prediction error signal. Neuron, 2005. 47(1): p. 129-41.

75. Juckel, G., et al., Dysfunction of ventral striatal reward prediction in schizophrenia. Neuroimage, 2006. 29(2): p. 409-16.

76. Jensen, J., et al., The formation of abnormal associations in schizophrenia: neural and behavioral evidence. Neuropsychopharmacology, 2008. 33(3): p. 473-9.

77. Murray, G.K., et al., Substantia nigra/ventral tegmental reward prediction error disruption in psychosis. Molecular Psychiatry, 2008. 13(3): p. 239, 267-76.

78. Arnsten, A.F.T., Catecholamine and second messenger influences on prefrontal cortical networks of "representational knowledge": a rational bridge between genetics and the symptoms of mental illness. Cerebral Cortex, 2007. 17: p. 6-15.

79. Goldman-Rakic, P.S., The "psychic" neuron of the cerebral cortex. Ann N Y Acad Sci, 1999. 868: p. 13-26.

80. Abi-Dargham, A., et al., Prefrontal dopamine D1 receptors and working memory in schizophrenia. Journal of Neuroscience, 2002. 22(9): p. 3708-19.

81. Goldman-Rakic, P.S., et al., Targeting the dopamine D1 receptor in schizophrenia: insights for cognitive dysfunction. Psychopharmacology (Berl), 2004. 174(1): p. 3-16.

82. Abi-Dargham, A. and H. Moore, Prefrontal DA transmission at DI receptors and the pathology of schizophrenia. Neuroscientist, 2003. 9(5): p. 404-16.

83. Erritzoe, D., et al., Positron emission tomography and single photon emission CT molecular imaging in schizophrenia. Neuroimaging Clin N Am, 2003. 13(4): p. 817-32.

84. Deutch, A.Y., The regulation of subcortical dopamine systems by the prefrontal cortex: interactions of central dopamine systems and the pathogenesis of schizophrenia. J Neural Transm Suppl, 1992. 36: p. $61-89$.

85. Deutch, A.Y., W.A. Clark, and R.H. Roth, Prefrontal cortical dopamine depletion enhances the responsiveness of mesolimbic dopamine neurons to stress. Brain Research, 1990. 521: p. $311-315$.

86. Meyer-Lindenberg, A., et al., Reduced prefrontal activity predicts exaggerated striatal dopaminergic function in schizophrenia. Nat Neurosci, 2002. 5(3): p. 267-71.

87. Kellendonk, C., et al., Transient and selective overexpression of dopamine D2 receptors in the striatum causes persistent abnormalities in prefrontal cortex functioning. Neuron, 2006. 49(4): p. 603-15.

88. Bach, M.E., et al., Transient and selective overexpression of D2 receptors in the striatum causes persistent deficits in conditional associative learning. Proceedings of the National Academy of Sciences, 2008. 105(41): p. 16027-32.

89. Drew, M.R., et al., Transient overexpression of striatal D2 receptors impairs operant motivation and interval timing. Journal of Neuroscience, 2007. 27(29): p. 7731-9. 
90. Maldonado, R., O. Valverde, and F. Berrendero, Involvement of the endocannabinoid system in drug addiction. Trends Neurosci, 2006. 29(4): p. 225-32.

91. Lupica, C.R. and A.C. Riegel, Endocannabinoid release from midbrain dopamine neurons: a potential substrate for cannabinoid receptor antagonist treatment of addiction. Neuropharmacology, 2005. 48(8): p. $1105-16$.

92. Cheer, J.F., et al., Phasic dopamine release evoked by abused substances requires cannabinoid receptor activation. Journal of Neuroscience, 2007. 27(4): p. 791-5.

93. Chen, J., et al., Delta 9-tetrahydrocannabinol enhances presynaptic dopamine efflux in medial prefrontal cortex. Eur J Pharmacol, 1990. 190(1-2): p. 259-62.

94. Tanda, G., F.E. Pontieri, and G. Di Chiara, Cannabinoid and heroin activation of mesolimbic dopamine transmission by a common mul opioid receptor mechanism. Science, 1997. 276(5321): p. 2048-50.

95. Cheer, J.F., et al., Cannabinoids enhance subsecond dopamine release in the nucleus accumbens of awake rats. Journal of Neuroscience, 2004. 24(18): p. 4393-400.

96. Melis, M., et al., Prefrontal cortex stimulation induces 2-arachidonoyl-glycerol-mediated suppression of excitation in dopamine neurons. J Neurosci, 2004. 24(47): p. 10707-15.

97. Matyas, F., et al., Identification of the sites of 2-arachidonoylglycerol synthesis and action imply retrograde endocannabinoid signaling at both GABAergic and glutamatergic synapses in the ventral tegmental area. Neuropharmacology, 2008. 54(1): p. 95-107.

98. Cheer, J.F., et al., Cannabinoids enhance subsecond dopamine release in the nucleus accumbens of awake rats. J Neurosci, 2004. 24(18): p. 4393-400.

99. French, E.D., K. Dillon, and X. Wu, Cannabinoids excite dopamine neurons in the ventral tegmentum and substantia nigra. Neuroreport, 1997. 8(3): p. 649-52.

100. Riegel, A.C. and C.R. Lupica, Independent presynaptic and postsynaptic mechanisms regulate endocannabinoid signaling at multiple synapses in the ventral tegmental area. J Neurosci, 2004. 24(49): p. $11070-8$.

101. Bossong, M.G., et al., Delta 9-tetrahydrocannabinol induces dopamine release in the human striatum. Neuropsychopharmacology, 2009. 34(3): p. 759-66.

102. Stokes, P.R., et al., Can recreational doses of THC produce significant dopamine release in the human striatum? Neuroimage, 2009.

103. Voruganti, L.N., et al., Cannabis induced dopamine release: an in-vivo SPECT study. Psychiatry Res, 2001. 107(3): p. 173-7.

104. D'Souza, D.C., et al., Delta-9-tetrahydrocannabinol effects in schizophrenia: implications for cognition, psychosis, and addiction. Biol Psychiatry, 2005. 57(6): p. 594-608.

105. D'Souza, D.C., et al., Effects of haloperidol on the behavioral, subjective, cognitive, motor, and neuroendocrine effects of Delta-9-tetrahydrocannabinol in humans. Psychopharmacology (Berl), 2008. 198(4): p. 587-603.

106. Liem-Moolenaar, M., et al., Central nervous system effects of haloperidol on THC in healthy male volunteers. Journal of Psychopharmacology, 2010.

107. Kreitzer, A.C. and R.C. Malenka, Dopamine modulation of state-dependent endocannabinoid release and long-term depression in the striatum. The Journal of Neuroscience, 2005. 25: p. 10537-10545.

108. Hilario, M.R., et al., Endocannabinoid signaling is critical for habit formation. Front Integr Neurosci, 2007. 1: p. 6.

109. Yin, H.H., et al., Dynamic reorganization of striatal circuits during the acquisition and consolidation of a skill. Nat Neurosci, 2009. 12(3): p. 333-41.

110. Calabresi, P., et al., Dopamine-mediated regulation of corticostriatal synaptic plasticity. Trends in Neurosciences, 2007. 30(5): p. $211-9$.

111. Pawlak, V. and J.N. Kerr, Dopamine receptor activation is required for corticostriatal spike-timingdependent plasticity. J Neurosci, 2008. 28(10): p. 2435-46. 
112. Shen, W., et al., Dichotomous dopaminergic control of striatal synaptic plasticity. Science, 2008. 321 (5890): p. 848-51.

113. Gerdeman, G.L., J. Ronesi, and D.M. Lovinger, Postsynaptic endocannabinoid release is critical to long-term depression in the striatum. Nat Neurosci, 2002. 5(5): p. 446-51.

114. Robbe, D., et al., Endogenous cannabinoids mediate long-term synaptic depression in the nucleus accumbens. Proc Natl Acad Sci U S A, 2002. 99(12): p. 8384-8.

115. Ronesi, J., G.L. Gerdeman, and D.M. Lovinger, Disruption of endocannabinoid release and striatal long-term depression by postsynaptic blockade of endocannabinoid membrane transport. J Neurosci, 2004. 24(7): p. 1673-9.

116. Kreitzer, A.C. and R.C. Malenka, Dopamine modulation of state-dependent endocannabinoid release and long-term depression in the striatum. J Neurosci, 2005. 25(45): p. 10537-45.

117. Yin, H.H. and D.M. Lovinger, Frequency-specific and D2 receptor-mediated inhibition of glutamate release by retrograde endocannabinoid signaling. Proc Natl Acad Sci U S A, 2006. 103(21): p. 8251-6.

118. Kreitzer, A.C. and R.C. Malenka, Endocannabinoid-mediated rescue of striatal LTD and motor deficits in Parkinson's disease models. Nature, 2007. 445(71 28): p. 643-7.

119. Schultz, W., Getting formal with dopamine and reward. Neuron, 2002. 36(2): p. 241 -63.

120. Broderick, P. and A.B. Benjamin, Caffeine and psychiatric symptoms: a review. The Journal of the Oklahoma State Medical Association, 2004. 97(12): p. 538-42.

121. Hedges, D.W., F.L. Woon, and S.P. Hoopes, Caffeine-induced psychosis. CNS Spectr, 2009. 14(3): p. $127-9$.

122. Andersen, M.B., et al., The adenosine A2A receptor agonist CGS 21680 exhibits antipsychotic-like activity in Cebus apella monkeys. Behavioural Pharmacology, 2002. 13(8): p. 639-44.

123. Weiss, S.M., et al., Potential for antipsychotic and psychotomimetic effects of A2A receptor modulation. Neurology, 2003. 61(11 Suppl 6): p. S88-93.

124. Dickerson, F.B., et al., A double-blind trial of adjunctive allopurinol for schizophrenia. Schizophr Res, 2009. 109(1-3): p. 66-9.

125. Brunstein, M.G., et al., A clinical trial of adjuvant allopurinol therapy for moderately refractory schizophrenia. J Clin Psychiatry, 2005. 66(2): p. 213-9.

126. Akhondzadeh, S., A. Safarcherati, and H. Amini, Beneficial antipsychotic effects of allopurinol as addon therapy for schizophrenia: a double blind, randomized and placebo controlled trial. Prog Neuropsychopharmacol Biol Psychiatry, 2005. 29(2): p. 253-9.

127. Akhondzadeh, S., et al., Dipyridamole in the treatment of schizophrenia: adenosine-dopamine receptor interactions. J Clin Pharm Ther, 2000. 25(2): p. 131-7.

128. Pertwee, R.G., The diverse $C B(1)$ and $C B(2)$ receptor pharmacology of three plant cannabinoids: Delta(9)-tetrahydrocannabinol, cannabidiol and Delta(9)-tetrahydrocannabivarin. Br J Pharmacol, 2007.

129. Zuardi, A., et al., Cannabidiol for the treatment of psychosis in Parkinson's disease. J Psychopharmacol, 2008.

130. Bhattacharyya, S., et al., Opposite Effects of Delta-9-Tetrahydrocannabinol and Cannabidiol on Human Brain Function and Psychopathology. Neuropsychopharmacology, 2009.

131. Leweke, M., et al., Different effects of nabilone and cannabidiol on binocular depth inversion in Man. Pharmacol Biochem Behav, 2000. 66(1): p. 175-81.

132. Morrison, P.D. and R.M. Murray, From real-world events to psychosis: the emerging neuropharmacology of delusions. Schizophrenia Bulletin, 2009. 35(4): p. 668-74.

133. Verrico, C.D., J.D. Jentsch, and R.H. Roth, Persistent and anatomically selective reduction in prefrontal cortical dopamine metabolism after repeated, intermittent cannabinoid administration to rats. Synapse, 2003. 49(1): p. 61-6. 
134. Ranganathan, M. and D.C. D'Souza, The acute effects of cannabinoids on memory in humans: a review. Psychopharmacology (Berl), 2006. 188(4): p. 425-44.

135. Lundqvist, T., Cognitive consequences of cannabis use: comparison with abuse of stimulants and heroin with regard to attention, memory and executive functions. Pharmacol Biochem Behav, 2005. 81(2): $p$ $319-30$

136. Solowii, N. and P.T. Michie, Cannabis and cognitive dysfunction: parallels with endophenotypes of schizophrenia? Journal of Psychiatry and Neuroscience, 2007. 32(1): p. 30-52.

137. Pistis, M., et al., Effects of cannabinoids on prefrontal neuronal responses to ventral tegmental area stimulation. Eur J Neurosci, 2001. 14(1): p. 96-102.

138. Pistis, M., et al., Delta(9)-tetrahydrocannabinol decreases extracellular GABA and increases extracellular glutamate and dopamine levels in the rat prefrontal cortex: an in vivo microdialysis study. Brain Res, 2002. 948(1-2): p. 155-8.

139. Jentsch, J.D., et al., Delta 9-tetrahydrocannabinol increases prefrontal cortical catecholaminergic utilization and impairs spatial working memory in the rat: blockade of dopaminergic effects with HA966. Neuropsychopharmacology, 1997. 16(6): p. 426-32.

140. Jentsch, J.D., et al., Repeated exposure to delta 9-tetrahydrocannabinol reduces prefrontal cortical dopamine metabolism in the rat. Neuroscience Letters, 1998. 246(3): p. 169-72.

141. Verrico, C.D., et al., Repeated, intermittent delta(9)-tetrahydrocannabinol administration to rats impairs acquisition and performance of a test of visuospatial divided attention. Neuropsychopharmacology, 2004. 29(3): p. 522-9.

142. Akil, M., et al., Catechol-O-methyltransferase genotype and dopamine regulation in the human brain. Journal of Neuroscience, 2003. 23(6): p. 2008-13.

143. Egerton, A., et al., Cannabinoids and prefrontal cortical function: insights from preclinical studies. Neurosci Biobehav Rev, 2006. 30(5): p. 680-95.

144. Herkenham, M., et al., Cannabinoid receptor localization in brain. Proc Natl Acad Sci U S A, 1990. 87(5): p. 1932-6.

145. Varvel, S.A. and A.H. Lichtman, Evaluation of CB 1 receptor knockout mice in the Morris water maze. J Pharmacol Exp Ther, 2002. 301(3): p. 915-24.

146. Cadoni, C., et al., Behavioural sensitization after repeated exposure to Delta 9-tetrahydrocannabinol and cross-sensitization with morphine. Psychopharmacology (Berl), 2001. 158(3): p. 259-66.

147. Cadoni, C., V. Valentini, and G. Di Chiara, Behavioral sensitization to delta 9-tetrahydrocannabinol and cross-sensitization with morphine: differential changes in accumbal shell and core dopamine transmission. Journal of Neurochemistry, 2008. 106(4): p. 1586-93.

148. Myin-Germeys, I., P. Delespaul, and J. van Os, Behavioural sensitization to daily life stress in psychosis. Psychological Medicine, 2005. 35(5): p. 733-41.

149. O'Daly, O., et al., Schizophrenia and substance abuse co-morbidity: a role for dopamine sensitization? Journal of Dual Diagnosis, 2005. 1(2): p. 11-40.

150. Lodge, D.J. and A.A. Grace, Amphetamine activation of hippocampal drive of mesolimbic dopamine neurons: a mechanism of behavioral sensitization. J Neurosci, 2008. 28(31): p. 7876-82.

151. Boileau, I., et al., Modeling sensitization to stimulants in humans: an [1 1C]raclopride/positron emission tomography study in healthy men. Archives of General Psychiatry, 2006. 63(12): p. 1386-95.

152. Chen, J.C., et al., Significance of glutamate and dopamine neurons in the ventral pallidum in the expression of behavioral sensitization to amphetamine. Life Sci, 2001. 68(9): p. 973-83.

153. Patrick, S.L., et al., Concomitant sensitization of amphetamine-induced behavioral stimulation and in vivo dopamine release from rat caudate nucleus. Brain Res, 1991. 538(2): p. 343-6.

154. Robinson, T.E. and J.B. Becker, Behavioral sensitization is accompanied by an enhancement in amphetamine-stimulated dopamine release from striatal tissue in vitro. European Journal of Pharmacology, 1982. 85(2): p. 253-4.

155. de Jong, J.G., et al., A single social defeat induces short-lasting behavioral sensitization to amphetamine. Physiol Behav, 2005. 83(5): p. 805-11. 
156. Kikusui, T., S. Faccidomo, and K.A. Miczek, Repeated maternal separation: differences in cocaineinduced behavioral sensitization in adult male and female mice. Psychopharmacology (Berl), 2005. 178(2-3): p. 202-10.

157. Sorg, B.A. and P.W. Kalivas, Effects of cocaine and footshock stress on extracellular dopamine levels in the ventral striatum. Brain Res, 1991. 559(1): p. 29-36.

158. Prasad, B.M., et al., Sensitization to stress and psychostimulants. Involvement of dopamine transmission versus the HPA axis. Ann N Y Acad Sci, 1995. 771: p. 617-25.

159. Lamarque, S., K. Taghzouti, and H. Simon, Chronic treatment with Delta(9)-tetrahydrocannabinol enhances the locomotor response to amphetamine and heroin. Implications for vulnerability to drug addiction. Neuropharmacology, 2001. 41(1): p. 118-29.

160. MacLean, K.I. and J.M. Littleton, Environmental stress as a factor in the response of rat brain catecholamine metabolism to delta8-tetrahydrocannabinol. European Journal of Pharmacology, 1977. $41(2)$ : p. $171-82$.

161. Mokler, D.J., et al., Neonatal administration of delta-9-tetrahydrocannabinol (THC) alters the neurochemical response to stress in the adult Fischer-344 rat. Neurotoxicol Teratol, 1987. 9(4): p. $321-7$.

162. Filip, M., et al., Involvement of cannabinoid CB 1 receptors in drug addiction: effects of rimonabant on behavioral responses induced by cocaine. Pharmacol Rep, 2006. 58(6): p. 806-19.

163. Corbille, A.G., et al., Role of cannabinoid type 1 receptors in locomotor activity and striatal signaling in response to psychostimulants. J Neurosci, 2007. 27(26): p. 6937-47.

164. Thiemann, G., et al., The role of the CB1 cannabinoid receptor and its endogenous ligands, anandamide and 2-arachidonoylglycerol, in amphetamine-induced behavioural sensitization. Behav Brain Res, 2008. 187(2): p. 289-96.

165. Thiemann, G., et al., The $C B(1)$ cannabinoid receptor antagonist AM251 attenuates amphetamineinduced behavioural sensitization while causing monoamine changes in nucleus accumbens and hippocampus. Pharmacol Biochem Behav, 2008. 89(3): p. 384-91. 



\section{DELTA-9-TETRAHYDROCANNABINOL-INDUCED DOPAMINE RELEASE AS A FUNCTION OF PSYCHOTIC ILLNESS AND PSYCHOTIC VULNERABILITY: [18F]FALLYPRIDE PET STUDY}

Rebecca Kuepper ${ }^{1}$, Jenny Ceccarini ${ }^{3}$, Johan Lataster ${ }^{1,4}$, Jim van Os ${ }^{1,5}$, Koen Van Laere ${ }^{3}$, Marinus van Kroonenburgh6, Joop van Gerven7, Machteld Marcelis ${ }^{1}$ \& Cécile Henquet ${ }^{1,2}$

1 Department of Psychiatry and Neuropsychology, South Limburg Mental Health Research and Teaching Network, EURON, Maastricht University, Maastricht, The Netherlands 2 PsyQ Heerlen, Mondriaan, South Limburg, The Netherlands ${ }^{3}$ Division of Nuclear Medicine, University Hospital and Catholic University Leuven, Belgium

${ }^{4}$ Open University of the Netherlands, Faculty of Psychology, Heerlen, The Netherlands ${ }^{5}$ King's College London, King's Health Partners, Department of Psychosis Studies, Institute of Psychiatry, London, UK

${ }^{6}$ Division of Nuclear Medicine, Maastricht University Medical Center, Maastricht, The Netherlands

7 Centre for Human Drug Research, Leiden, The Netherlands submitted 


\begin{abstract}
Cannabis use is associated with psychosis, particularly in those with expression of, or vulnerability for, psychotic illness. The biological underpinnings of these differential associations, however, remain largely unknown. We tested the hypothesis that THC (delta-9-tetrahydrocannabinol, the main psychoactive ingredient of cannabis) provokes psychotic experiences by differential induction of striatal dopamine release. Positron Emission Tomography and [18F]fallypride were used to investigate striatal dopamine release after pulmonary administration of THC in a group of healthy cannabis users (average risk psychotic disorder), patients with psychotic disorder (high risk psychotic disorder) and first-degree relatives (intermediate risk psychotic disorder). While THC was not associated with increased dopamine release in the control group, significant ligand displacement in striatal subregions indicative of dopamine release was detected in both patients and relatives, most pronounced in caudate nucleus. The results indicate that dopamine may mediate the psychotogenic effects of THC, in particular in individuals already at risk for dopamine dysregulation.
\end{abstract}




\section{Introduction}

The use of cannabis, the most frequently used illicit drug in the world, has long been associated with an increased risk of developing psychotic symptoms in healthy individuals, and with poor outcome in patients with psychotic disorder [1-4]. While the epidemiological link between cannabis and psychosis is well established, very little is known about the biological underpinnings of this association $[5,6]$.

Long-term heavy cannabis use, in particular when started during early adolescence, is associated with structural brain changes such as impaired structural integrity of the corpus callosum [7], alterations in white and gray matter [8, 9], and decreased hippocampal and amygdala volumes [10]. However, it has been argued that the use of cannabis is unlikely to increase the risk of psychosis by mechanisms that manifest themselves as major structural brain changes [1 1, 12]. Alternatively, neurochemical interactions between cannabis and neurotransmitters such as dopamine (DA) may constitute a biological link between cannabis and psychosis [6]. In the human brain, delta-9-tetrahydrocannabinol (THC, the main psychoactive constituent of cannabis) binds to cannabinoid $1(C B 1)$ receptors, the main binding site of endogenous cannabinoids (eCBs) $[13,14]$. Activation of $C B 1$ receptors inhibits pre-synaptic neurotransmitter release - direct targets being GABA and glutamate terminals- and consequently modulates several neurotransmitter systems, including the DA system $[13,15,16]$. DA is thought to play a role in schizophrenia pathophysiology $[17,18]$, and animal studies suggest that THC affects DA neurotransmission in several regions of the brain including prefrontal cortex (PFC) and mesolimbic regions [19-22]. However, direct evidence for interaction between THC and DA in the human brain to date remains scarce. First insights came from a single case report study with Single Positron Emission Computed Tomography (SPECT) and the radiotracer [123|]IBZM. In this study, a $20 \%$ decrease in striatal D2 receptor binding ratio was observed, indicating increased synaptic DA activity, in a medication-free patient with schizophrenia just after using cannabis [23]. Three subsequent studies used neurochemical imaging to examine the effects of THC on DA neurotransmission in healthy human volunteers. Bossong and colleagues [24] included seven healthy male recreational cannabis users and investigated the effects of THC on DA neurotransmission with Positron Emission Tomography (PET) and [ $\left.{ }^{11} \mathrm{C}\right]$ raclopride. The authors observed small (around 3.5\%) but significant decreases in D2 receptor binding in two subregions of the striatum, the ventral striatum and the precommissural dorsal putamen after pulmonary THC administration [24]. The PET study by Stokes and colleagues [25] failed to find significant changes in D2 receptor binding after an oral dose of THC in thirteen healthy male volunteers. Similarly, Barkus and colleagues [26] did not observe DA release after intravenous THC studied with SPECT and [123|]IBZM [26]. 
The present study aimed to shed light on these inconsistencies, hypothesizing that inconsistencies in previous studies may be related to differential inclusion of individuals with increased sensitivity to THC. Thus, we not only investigated THCinduced DA release using PET and the highly selective and high affinity DA $D_{2 / 3}$ PET radioligand [ $\left.{ }^{18} \mathrm{~F}\right]$ fallypride (Lataster and colleagues, [27]) in a group of healthy cannabis users, but also in groups with demonstrated increased sensitivity to THC: patient with psychotic disorder and their first-degree relatives. Patients with psychotic disorder have been shown to be more sensitive to the behavioral and cognitive effects of cannabis [28, 29] and individual differences in sensitivity to cannabis are in part mediated by genetic risk for psychotic disorder, siblings displaying more sensitivity than well controls $[30,31]$.

\section{Materials and methods}

\section{Participants}

A total of 30 volunteers (10 patients with psychotic disorder, 10 first-degree relatives of patients with psychotic disorder, and 10 healthy controls agreed to participate in the study. Participants were recruited through flyers in local coffee shops (cafes where cannabis is sold and consumed legally) and newspaper advertisements, as well as through in- and outpatient mental health service facilities in South Limburg, The Netherlands. Inclusion criteria were i) age 18-60 years, ii) sufficient command of the Dutch language, iii) no intellectual impairment (i.e. $I Q>$ 80 ) as ensured by the Dutch version of the Wechsler Adult Intelligence Scale [32], iv) having smoked cannabis at least once in the past 12 months, v) patients only: a diagnosis of psychotic disorder according to the Diagnostic and Statistical Manual of Mental Disorders (DSM-IV) [33], and vi) relatives only: having a first degree relative with a diagnosis of psychotic disorder. Exclusion criteria were i) head trauma with loss of consciousness or neurological disorder, ii) endocrine or cardiovascular disorder, iii) a diagnosis of psychiatric illness according to the DSM-IV in the relatives and the controls and, controls only, a positive family history of psychotic disorder, iv) current use of psychotropic medication, v) current use of illicit drugs other than cannabis, vi) current use of alcohol in excess of 5 standard units per day, vii) presence of metal elements in the body, viii) pregnancy or lactation, and ix) a history of claustrophobia.

The study was carried out in accordance with the World Medical Association's declaration of Helsinki and approved by the standing medical ethics committee of Maastricht University Medical Center. Written informed consent was obtained from all participants. 


\section{Design and procedure}

The study made use of a single-blind placebo-controlled design. This was done in order to apply statistical modeling as previously described by Christian and colleagues [34] and recently by Lataster and colleagues [27], which requires only a single radiochemical synthesis and administration, thereby avoiding session effects and minimizing the amount of radiation exposure. All participants attended a screening session, during which inclusion and exclusion criteria were confirmed and baseline clinical measures taken. During the actual testing session, which was separated by approximately one week from the screening session, participants received both $8 \mathrm{mg}$ of THC and placebo in a single-blind manner: participants first received the placebo followed by the active drug separated by approximately 2 hours, but were told that the order of administration was random. Participants were asked to abstain from cannabis at least five days prior to the testing session and from caffeine and nicotine 4 hours prior to the testing session. At the beginning of the testing session, participants received a standardized meal and a caffeine-free beverage. Baseline clinical measures were repeated in case the screening session and testing session were more than one week from each other. Urinalysis was carried out to verify drug and medication abstinence and a pregnancy test was done to rule out pregnancy in the female participants. Additionally, abstinence from alcohol was assured by means of a breathalyzer. Finally, the inhalation procedure was practiced. The PET procedure started approximately 1 hour after the start of the testing session (see figure 1 for an illustration of the PET protocol). Effects of THC on subjective experience and psychopathology were assessed using computerassisted tasks and self-report questionnaires, which were applied after the administration of placebo and THC, respectively. At the end of each session, blood pressure and heart rate was measured. All participants stayed under psychological observation until the acute effects of the THC had faded and it was safe for the participants to return home.

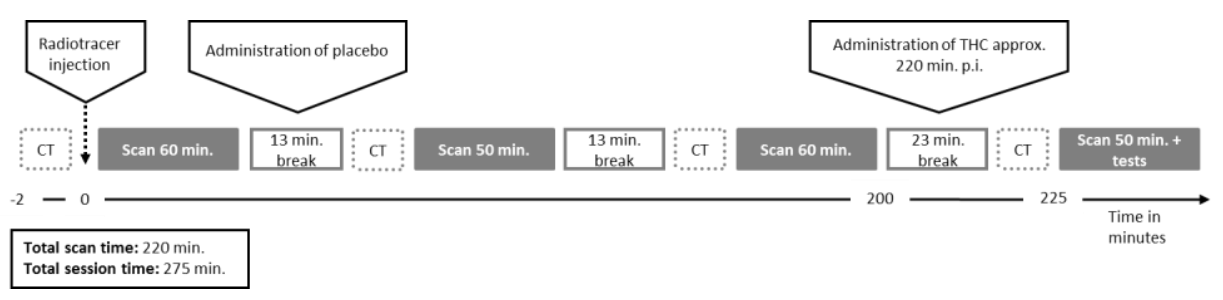

Figure 1. Schematic illustration of the scan protocol. The first three scan segments provided a total of 53 frames, representing tracer kinetics during the baseline condition; the 25 frames of the last scan segment represented tracer kinetics during the exposure condition. Each scan segment was preceded by a 2 min CT scan and followed by a short break. 


\section{Baseline clinical measures}

Diagnoses were confirmed using the Operational Criteria Checklist and associated OPCRIT computer program [35]. Presence and severity of psychotic symptoms during the past two weeks was assessed in all participants with the Positive and Negative Syndrome Scale (PANSS) [36]. Current and past cannabis and other drug use was assessed using the appropriate sections of the WHO Composite International Diagnostic Interview [37] and the Structured Clinical Interview for DSM Disorders [38].

\section{Drug preparation and administration}

Preparation and administration of drugs was performed according to Zuurman and colleagues [39]. THC was purified from Cannabis sativa by Farmalyse BV, Zaandam, The Netherlands, in agreement with GMP guidelines, and was dissolved in $200 \mu \mathrm{l}$ 100 vol\% alcohol. The solvent was used as placebo. Drugs were administered by means of a vaporizer (Volcano ${ }^{\circledR}$, Storz-Bickel GmbH, Tuttlingen, Germany). Cannabis vaporization is a technology designed to safely and effectively deliver THC while avoiding the respiratory hazards of smoking by heating THC to a temperature where active THC vapors are produced, but below the point of combustion where noxious pyrolytic by products are formed [40, 41]. Approximately five minutes before administration, $8 \mathrm{mg}$ of THC was vaporized and stored in an opaque polythene bag equipped with a valved mouthpiece preventing the loss of THC in between inhalations. As practiced at the beginning of the testing session, subjects were instructed to inhale the volume of the bag in 3-5 subsequent inhalations, holding their breath for 10 seconds after each inhalation and without speaking during the inhalation process. The same procedure was followed for the administration of placebo.

\section{Blood sampling}

Venous blood samples were taken at baseline and 5, 10, 15, and 75 minutes after THC administration to determine plasma concentrations of THC and its two main metabolites 11 -OH-THC and 11 -nor-9-carboxy-THC, as indicated by Zuurman and colleagues [39, 42]. To prevent unblinding of participants, sham samples were taken at baseline and 5, 10, 15 and 75 minutes after placebo administration. Plasma samples were analyzed by ABL, Analytisch Biochemisch Laboratorium BV, Assen, The Netherlands.

\section{Visual Analogue Scales}

For experimental validation, Visual Analogue Scales (VAS) on alertness, feeling 'high' and external and internal perception [39, 43] were used to assess subjective changes in perception induced by THC. 


\section{PET scans}

\section{Radiotracer preparation}

The fluorinated substituted benzamide $\left[{ }^{18} \mathrm{~F}\right]$ fallypride is a high affinity antagonist radiotracer used to visualize and estimate both striatal and extrastriatal DA D2/3 receptors $[44,45]$. The use of $\left[{ }^{18} \mathrm{~F}\right]$ fallypride in combination with the linear extension of the simplified reference region model as described by Alpert and colleagues [46] allows for a single experimental session requiring only a single radiochemical synthesis and administration, baring the advantage of avoiding session effects and minimizing amount of radiation exposure for the participants as previously demonstrated in an environmental exposure paradigm [27]. The precursor for tracer synthesis was obtained from ABX (Radeberg, Germany) and labeling was performed on-site using a Raytest Synchrom R\&D synthesis module (Raytest, Straubenhardt, Germany). The final product was obtained after reverse-phase high performance liquid chromatographic (HLPC) purification using a Waters XTerra ${ }^{\mathrm{TM}}$ RP18 $5 \mu \mathrm{m} 7.8 \mathrm{~mm} \times 150 \mathrm{~mm}$ column and sodium acetate $0.05 \mathrm{M} \mathrm{pH5.5/ethanol}$ $70: 30 \mathrm{~V} / \mathrm{V}$ as mobile phase at a flow rate of $1.5 \mathrm{ml} / \mathrm{min}$. The [ $\left.{ }^{18} \mathrm{~F}\right]$ fallypride eluted after 18 minutes. The collected peak $(2 \mathrm{ml})$ was diluted with $8 \mathrm{ml}$ of $\mathrm{NaCl} 0.9 \%$ and sterile filtered over a Millipore Cathivex-GS $0.22 \mu \mathrm{m}$ filter. The final product of the radioligand was administered as a sterile solution of $7 \mathrm{mM}$ sodium acetate buffer $\mathrm{pH}$ $5.5,0.72 \%$ and $6 \%$ ethanol. The specific radioactivity at the time of injection was greater than $37 \mathrm{GBq} / \mu \mathrm{mol}(1000 \mathrm{Ci} / \mathrm{mmol})$. Radiochemical purity was $>95 \%$.

\section{Data acquisition and processing}

Subjects were placed on the scanner bed with their head fixated using a vacuum bed and the body strapped to the bed to avoid movement during PET acquisition. Positions of the monitor and response box were adjusted to allow for optimal comfort. Subjects received $185 \mathrm{MBq}$ of [ $\left.{ }^{18} \mathrm{~F}\right]$ fallypride in a slow intravenous bolus injection through a catheter in the left antecubital vein. Upon tracer injection, dynamic emission scans were initiated in three-dimensional mode using a PET/CT scanner (Philips, Eindhoven, The Netherlands). Data were acquired in frames of 60 seconds during the first 6 minutes and in frames of 120 seconds thereafter. PET emission was performed based on the PET imaging protocol for [ $\left.{ }^{18} \mathrm{~F}\right]$ fallypride used previously by Christian and colleagues [34], adapted for detecting [18F]fallypride displacement in striatal brain regions according to a simulation study (Ceccarini et al., submitted). Emission data were acquired in four segments, separated by a total of three breaks (figure 1). Given the use of an "activation" parameter in the kinetic model used for analyses (Alpert et al., 2003; discussed below), representing presence or absence of additional DA release, and the hypothesis of THC administration being associated with increased DA activity, the THC condition was always presented after the placebo condition. The first three PET segments with a 
total duration of 170 min thus represented tracer kinetics during the placebo condition. Administration of placebo occurred between the first and the second segment. THC was administered at 220 min post-injection, followed by the last PET segment with duration of $50 \mathrm{~min}$, representing tracer kinetics during the exposure condition. The timing of THC administration was adjusted such that the proportional distribution of radioligand was optimized for striatal brain regions, high in dopamine $D_{2 / 3}$ receptor density [34]. Each PET segment was preceded by a lowdose CT scan (80kV tube potential, $11 \mathrm{mAs}$ ) to correct for attenuation. PET data were reconstructed using a 3D OSEM (ordered-subset expectation maximization) iterative reconstruction including model-based scatter as well as attenuation correction based on a measured attenuation map acquired by the $\mathrm{CT}$, with a final spatial resolution of $4 \mathrm{~mm}$. All subjects additionally received a high-resolution $\mathrm{T} 1$ weighted structural MRI for anatomical co-registration purposes (1.5 Tesla Scanner, Phillips, Netherlands). Reconstructed PET data were transferred in DICOM (Digital Imaging and Communications in Medicine) and converted to Analyze using PMOD software version 2.95 (PMOD Inc., Zurich Switzerland). To correct for head motion during the scan segments, all PET frames were realigned, co-registered to the subject's MRI and then spatially normalized to a specific T1-weighted template constructed in MNI (Montreal Neurological Institute) stereotaxic space using SPM2 (Statistical Parametric Mapping, The Wellcome Department of Cognitive Neurology, London, UK). To increase signal to noise ratio, the normalized images were then smoothed with a 3D gaussian filter ( $4 \mathrm{~mm}$ full width at half maximum) before applying the pharmacokinetic model.

\section{Statistical analysis}

Given hierarchical clustering of the data, each person contributing minimally two observations (placebo and THC), behavioral (VAS) data were analyzed using multilevel random regression analysis in Stata using the XTREG routine, examining the effects of condition (placebo versus THC) on subjective experience.

To estimate THC-induced DA release, PET data were analyzed applying the linear extension of the simplified reference region model (LSRRM) [46], a kinetic model described and used in functional neurotransmitter [ $\left.{ }^{18} \mathrm{~F}\right]$ fallypride PET studies on DA D2/3 receptors [34, 47]. This method takes into account the THC-induced temporal perturbations in ligand specific binding, including a baseline condition and a DA activation paradigm during a single scan session. The design has several practical advantages, such as the requirement for only a single radiochemical synthesis and administration and avoidance of session effects. The LSRRM accounts for timedependent changes in ligand concentration, assuming that the steady state is not maintained. The LSRRM therefore allows the dissociation rate of the ligand, $k 2 a$, to 
change throughout the paradigm in response to fluctuating levels of DA $\left(k_{2 a}=k_{2} /[1\right.$ $+B P_{N D}$, where $k_{2}$ is the tissue to plasma efflux constant and BP $P_{N D}$ is the nondisplaceable binding potential). Changes in $B P_{N D}$ in activation studies are usually assumed to reflect changes in the concentration of available receptor sites ( $\left.B_{\text {avail }}\right)$, and a decrease in $B P_{N D}$ is assumed to reflect increased neurotransmitter release. The temporal change of $k_{2}$, via a change in $B P_{N D}$, is obtained by introducing the additional term $Y \cdot h(t)$, where $Y$ represents the amplitude of the ligand displacement and the function $h(t)$ describes a rapid change following activation onset and dissipation over time. The exponential decay function $h(t)=\exp [-T(t-T)]$ accounts for temporal variation in the model parameters, where $T$ controls the rate at which activation effects die away $\left(T=0.03 \mathrm{~min}^{-1}\right.$ ) and $\mathrm{T}$ indicates activation initiation ( $\mathrm{T}=$ $220 \mathrm{~min})$. It follows that, through linearization of the simplified reference region model (SRRM), an increased $k_{2}$ a reflected in a decreased BPND for DA receptors due to increased DA release would result in a positive value of $\mathrm{Y}$. The cerebellum, representing an area with negligible density of DA D2/3 receptors, was used as reference region [48]. For each subject, two binary masks were created based on the corresponding normalized MRI, using an in-house created set of volumes-ofinterests (VOIs) based on the Talairach atlas [49]. One binary mask contained all brain regions of interest (i.e. the striatal subregions, in particular caudate nucleus, putamen, pallidum and nucleus accumbens), and a second mask was drawn only on the cerebellum. For each subject, VOI analysis was performed by estimating the kinetic parameters using the LSRRM and the PET time-activity curves (TACs) for all VOls. A voxel-wise $t$-statistic map of the $Y$ parameters was computed to localize those areas with estimated increased DA release following THC administration. These statistic $t$ maps were generated as $t=\mathrm{Y} / \mathrm{sd}(\mathrm{Y})$, where the standard deviation parametric image of $\mathrm{Y}(\mathrm{sd}[\mathrm{Y}])$ was created based on the estimated covariance matrix, consistent with previous work by Christian and colleagues [34] and Lataster and colleagues [27]. The false discovery rate (FDR) [50] correction was utilized to control statistical significance thresholds in the context of multiple comparisons at the voxel level [51]. In order to identify the spatial extent of the neuromodulation for each region of interest showing significant activation-induced DA release, the percentage of voxels exceeding an FDR-corrected significance threshold of $p(\alpha(F D R)$ $=5 \%)<0.05$ was calculated. Group differences in the spatial extent of THCinduced DA release were then tested using regression models within STATA. Inspection of residuals from the regression models indicated substantial heteroscedasticity of the error variances across the three groups. To account for this, we used a regression model that allowed the error variances to differ between groups. All analyses were a priori adjusted for age (in years), sex, nicotine use (continuous: number of cigarettes per day), alcohol use (continuous: grams of alcohol per week) and use of other medication (dichotomous: yes versus no). 


\section{Results}

\section{Participants}

The initial sample consisted of 10 patients with a DSM-IV diagnosis of psychotic disorder, 10 first-degree relatives of patients with psychotic disorder, and 10 healthy controls. However, two patients were excluded due to protocol violation in terms of use of antipsychotic medication. In addition, two individuals (one relative and one control subject) were excluded due to excessive movement during the scan, yielding uncorrectable movement artifacts in the PET data. The resulting final sample thus consisted of 8 medication-free patients with a diagnosis of psychotic disorder, 9 first-degree relatives and 9 healthy controls. Of the patients, five individuals fulfilled criteria for non-affective psychotic disorder and three individuals fulfilled criteria for affective psychotic disorder. Controls and relatives did not fulfill criteria for any psychiatric disorder. There were not suggestive differences between three groups with regard to mean age, male/female ratio and mean intellectual functioning as indexed by $I Q$ (Table 1). Patients had higher scores on the positive syndrome dimension of the PANSS. All participants had used cannabis during the past 12 months: 16 participants reported daily use during the past 12 month, 3 participants admitted to weekly use and 7 participants used cannabis monthly or less than monthly (Table 1).

\section{Drug screening}

Urinalysis was positive for THC in 18 participants $(6$ patients, 6 relatives and 6 controls). Since the majority of the sample reported daily use of cannabis, urinalysis can be expected to reveal traceable amounts of $\mathrm{THC}_{\text {; }}$ all participants indicated compliance with the protocol and gave verbal confirmation of abstention from cannabis minimally 5 days before testing. One participant tested positive for cocaine (a relative). For power reasons and since exclusion of this latter individual did not change the results, this observation was kept in the analyses. All participants tested negative for benzodiazepines, amphetamine, methamphetamine, opiates and alcohol.

\section{Blood sample analysis}

The concentration of THC in plasma reached a maximum of $37.3 \pm 19.3 \mathrm{ng} / \mathrm{ml}$ at 5 minutes post inhalation and decreased subsequently. The two main metabolites 11 $\mathrm{OH}-\mathrm{THC}$ and $\mathrm{THC}-\mathrm{COOH}$ reached a maximum concentration of $1.6 \pm 1.5 \mathrm{ng} / \mathrm{ml}$ and $25.2 \pm 22.4 \mathrm{ng} / \mathrm{ml}$ at 5 and 15 minutes post-inhalation, respectively (see table 2 and figure 2). 
Table 1. Participant characteristics.

\begin{tabular}{|c|c|c|c|}
\hline & $\begin{array}{l}\text { Controls } \\
(n=9)\end{array}$ & $\begin{array}{c}\text { Relatives } \\
(n=9)\end{array}$ & $\begin{array}{c}\text { Patients } \\
(n=8)\end{array}$ \\
\hline Mean Age (SD) & $31.4(11.4)$ & $36.1(12.0)$ & $31.1(8.9)$ \\
\hline Percentage male (n) & $55.6(5)$ & $66.7(6)$ & $75.0(6)$ \\
\hline Mean IQ (SD) & $102.8(14.0)$ & 105.5 (11.8) & $102.7(15.6)$ \\
\hline \multicolumn{4}{|l|}{ Cannabis use $\%(n)^{*}$} \\
\hline Monthly or less & $33.3(3)$ & $22.2(2)$ & $25.0(2)$ \\
\hline Weekly & $11.1(1)$ & $11.1(1)$ & $12.5(1)$ \\
\hline Daily & $55.6(5)$ & $66.7(6)$ & $62.5(5)$ \\
\hline \multicolumn{4}{|l|}{ Mean PANSS score (SD) } \\
\hline Positive & $1.0(0.0)$ & $1.0(0.1)$ & $1.5(0.7)$ \\
\hline Negative & $1.0(0.0)$ & $1.0(0.1)$ & $1.1(0.2)$ \\
\hline Global & $1.2(0.1)$ & $1.1(0.1)$ & $1.2(0.2)$ \\
\hline Total & $1.0(0.0)$ & $1.0(0.1)$ & $1.1(0.1)$ \\
\hline \multicolumn{4}{|l|}{ Nicotine use ${ }^{a} \%(n)$} \\
\hline 0 & $44.4(4)$ & $22.2(2)$ & $25(2)$ \\
\hline $1-10$ & $22.2(2)$ & $44.4(4)$ & $25(2)$ \\
\hline $11-20$ & $33.3(3)$ & $33.3(3)$ & $37.5(3)$ \\
\hline$>20$ & $0.0(0)$ & $0.0(0)$ & $12.5(1)$ \\
\hline \multicolumn{4}{|l|}{ Alcohol use ${ }^{b} \%$ (n) } \\
\hline $0-50$ & $66.7(6)$ & $44.4(4)$ & $62.5(5)$ \\
\hline $50-150$ & $33.3(3)$ & $44.4(4)$ & $0.0(0)$ \\
\hline $150-350$ & $0.0(0)$ & $11.1(1)$ & $37.5(3)$ \\
\hline \multicolumn{4}{|l|}{ Other medication use $\%(n)$} \\
\hline Yes & $0.0(0)$ & $11.1(1)$ & $25.0(2)$ \\
\hline No & $100.0(9)$ & $88.9(8)$ & $75.0(6)$ \\
\hline \multicolumn{4}{|l|}{ Use of contraceptives \% (n) } \\
\hline Yes & $22.2(2)$ & $11.1(1)$ & $0.0(0)$ \\
\hline No & $77.8(7)$ & $88.9(8)$ & $100.0(8)$ \\
\hline
\end{tabular}

Note. Percentages do not always total 100 due to rounding.

* Refers to cannabis use in the last 12 month.

a Refers to number of cigarettes per day.

b Refers to grams per week. Standard drink/unit size in the Netherlands contains $9.9 \mathrm{~g}$ of ethanol.

Table 2. Mean maximum plasma concentration of $\mathrm{THC}, 11-\mathrm{OH}-\mathrm{THC}$ and $\mathrm{THC}-\mathrm{COOH}$

\begin{tabular}{r|ccc|} 
& $\begin{array}{c}\text { Patients } \\
(\mathbf{n}=\mathbf{9})\end{array}$ & $\begin{array}{c}\text { Relatives } \\
(\mathbf{n}=\mathbf{9})\end{array}$ & $\begin{array}{c}\text { Controls } \\
(\mathbf{n}=\mathbf{8})\end{array}$ \\
\hline THC & $43.5(22.4)$ & $36.1(19.3)$ & $33.0(17.0)$ \\
\hline $11-\mathrm{OH}-\mathrm{THC}$ & $1.6(1.3)$ & $2.2(2.0)$ & $1.2(0.9)$ \\
THC-COOH & $25.7(30.3)$ & $28.0(21.9)$ & $22.0(16.3)$
\end{tabular}

Note. Numbers are $\mathrm{ng} / \mathrm{ml}(\mathrm{SD})$. 


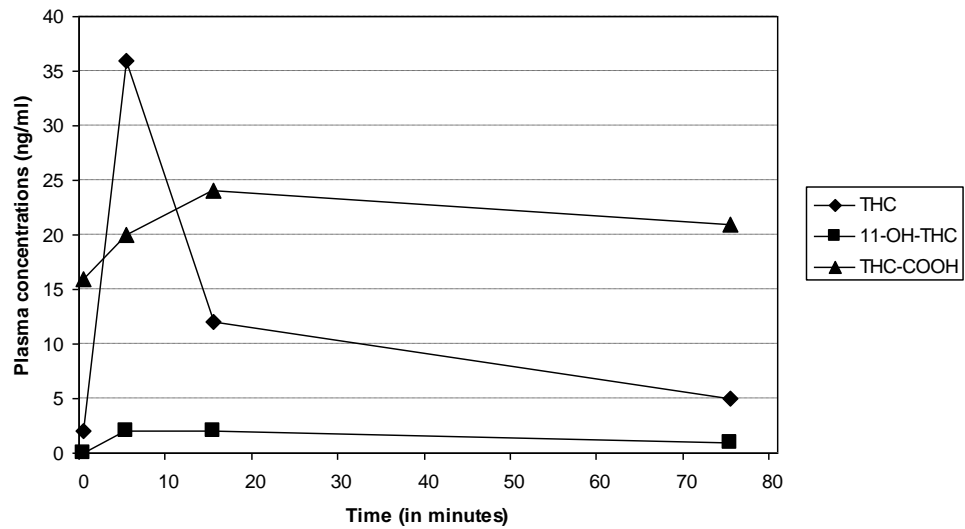

Figure 2. Plasma concentrations of THC and its two main metabolites $11-\mathrm{OH}-\mathrm{THC}$ and 11 -nor-9-carboxy-THC (THC-COOH) at baseline (0) and at 5, 15, and 75 minutes post inhalation across groups.

\section{Visual Analogue Scales}

From the 13 VAS composite scores on 'external perception' ( 5 scales) and 'internal perception' ( 7 scales) were calculated. The scale on 'feeling high' was analyzed separately. As expected, THC induced significant increases in 'feeling high' $(\beta=$ $11.74,95 \% \mathrm{Cl}: 6.90-16.59, p<0.001)$, 'external perception' $(\beta=2.16,95 \% \mathrm{Cl}$ : $0.84-3.47, p=0.001)$ and 'internal perception' $(\beta=1.19,95 \% \mathrm{Cl}: 0.01-2.38, p=$ 0.049 ) (figure 3).
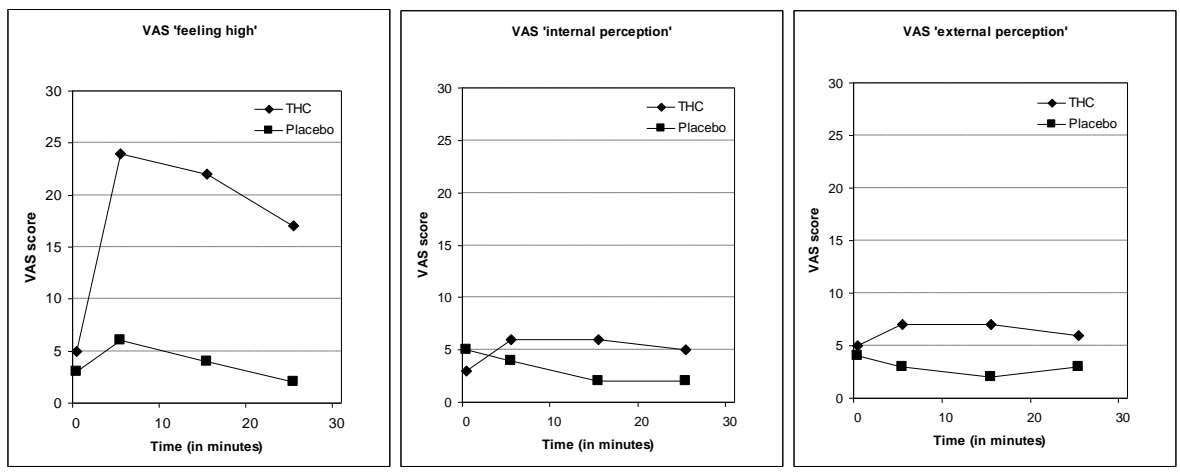

Figure 3. Effects of pulmonary THC on subjective feelings of 'high', 'internal perception', and 'external perception' across groups, measured with Visual Analogue Scales at baseline (0) and 5, 15, and 25 minutes post-inhalation. 


\section{In vivo DA release following THC administration}

Inspection of the scatterplot revealed one outlier (a relative); this subject was accordingly excluded from this analysis. THC induced detectable amounts of [ $\left.{ }^{18} \mathrm{~F}\right]$ fallypride displacement, indicative of DA release, throughout the striatum in both patients and relatives, but not in controls (see figure 4 and 5 and table 3). On average, $Y$ was positive, indicating DA release for all striatal subregions in the patient group, and for the caudate nucleus in the relatives (see table 4). Furthermore, there was a significant difference between the three groups with regard to the amount of ligand displacement in right and left caudate nucleus, left putamen, and right pallidum (see table 3 for group statistics). Post hoc pairwise comparisons showed that the amount of ligand displacement was significantly larger for patients and relatives versus controls, respectively, in left caudate nucleus ( $B_{\text {patients }}$ $\left.=0.18, p=0.002, B_{\text {relatives }}=0.18, p=0.021\right)$, left putamen $\left(B_{\text {patients }}=0.13, p=\right.$ $0.018, B_{\text {relatives }}=0.12, p=0.047$ ) and left pallidum ( $B_{\text {patients }}=0.19, p=0.032$, $\left.B_{\text {relatives }}=0.07, p=0.020\right)$. In the right caudate nucleus, the only group difference was between patients and controls $(B=0.18, p=0.032)$. No differences were observed between patients and relatives in any of the subregions. Tables 3 and 4 summarize THC-induced changes in $\left.{ }^{18} \mathrm{~F}\right]$ fallypride binding quantified as the number of voxels within a region exceeding the FDR-corrected significance threshold of $\left.P_{(\alpha(F D)}=5 \%\right)<0.05$, and mean parameter estimates, respectively. Ligand displacement was independent of age, gender, alcohol use, and nicotine use.

Table 3. Mean percentages (SD) of THC-induced ligand displacement in striatal subregions

\begin{tabular}{|c|c|c|c|c|}
\hline Caudate Nucleus & $\begin{array}{l}\text { Controls } \\
(n=9)\end{array}$ & $\begin{array}{c}\text { Relatives } \\
(n=9)\end{array}$ & $\begin{array}{l}\text { Patients } \\
\left(n=7^{a}\right)\end{array}$ & Group statistics* \\
\hline Right & $3.5(5.6)$ & $12.6(19.0)$ & $15.3(19.7)$ & $F(3,21)=2.18 ; p=0.071$ \\
\hline Left & $2.5(3.0)$ & $19.0(19.5)$ & $16.2(16.9)$ & $F(3,21)=1.67 ; p=0.058$ \\
\hline Average & $3.0(3.2)$ & $15.7(17.9)$ & $15.9(15.1)$ & $F(3,21)=2.48 ; p=0.034$ \\
\hline \multicolumn{5}{|l|}{ Putamen } \\
\hline Right & $1.4(1.5)$ & $9.8(17.3)$ & $16.2(19.8)$ & $F(3,21)=1.85 ; p=0.042$ \\
\hline Left & $2.4(4.3)$ & $14.6(16.7)$ & $13.7(12.7)$ & $F(3,21)=2.14 ; p=0.043$ \\
\hline Average & $1.9(2.5)$ & $12.3(14.9)$ & $14.9(15.5)$ & $F(3,21)=2.43 ; p=0.025$ \\
\hline \multicolumn{5}{|l|}{ Pallidum } \\
\hline Right & $1.5(2.7)$ & $6.8(7.6)$ & $16.4(21.8)$ & $F(3,21)=2.15 ; p=0.022$ \\
\hline Left & $3.3(5.6)$ & $15.7(27.9)$ & $11.6(17.1)$ & $F(3,21)=0.54 ; p=0.329$ \\
\hline Average & $2.3(2.8)$ & $10.8(15.5)$ & $14.4(15.6)$ & $F(3,21)=1.71 ; p=0.048$ \\
\hline \multicolumn{5}{|l|}{ Nucleus Accumbens } \\
\hline Right & $2.2(5.7)$ & $3.4(7.3)$ & $10.9(16.1)$ & $F(3,21)=1.37 ; p=0.086$ \\
\hline Left & $1.2(2.9)$ & $11.3(22.4)$ & $4.5(6.4)$ & $F(3,21)=0.81 ; p=0.440$ \\
\hline Average & $1.7(3.1)$ & $7.4(14.9)$ & $7.7(7.1)$ & $F(3,21)=1.51 ; p=0.140$ \\
\hline
\end{tabular}

Note. Numbers are percentages $(S D)$ of voxels within a region exceeding the FDR corrected significance threshold of $P_{(\alpha}(F D R)=$ $5 \%)<0.05$, relative to the total number of voxels within the mask of the respective region.

a One subject was identified as an outlier and accordingly excluded from this analysis.

*adjusted for age, gender, alcohol and nicotine use 


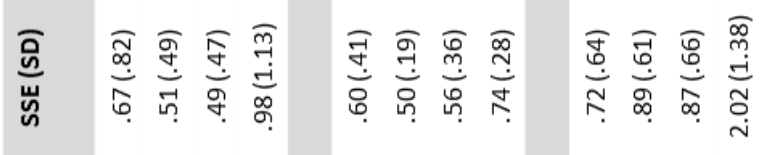

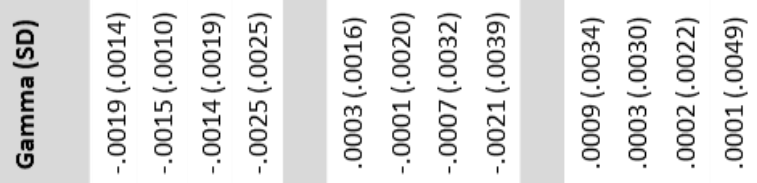

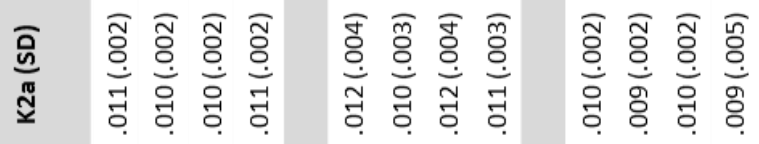

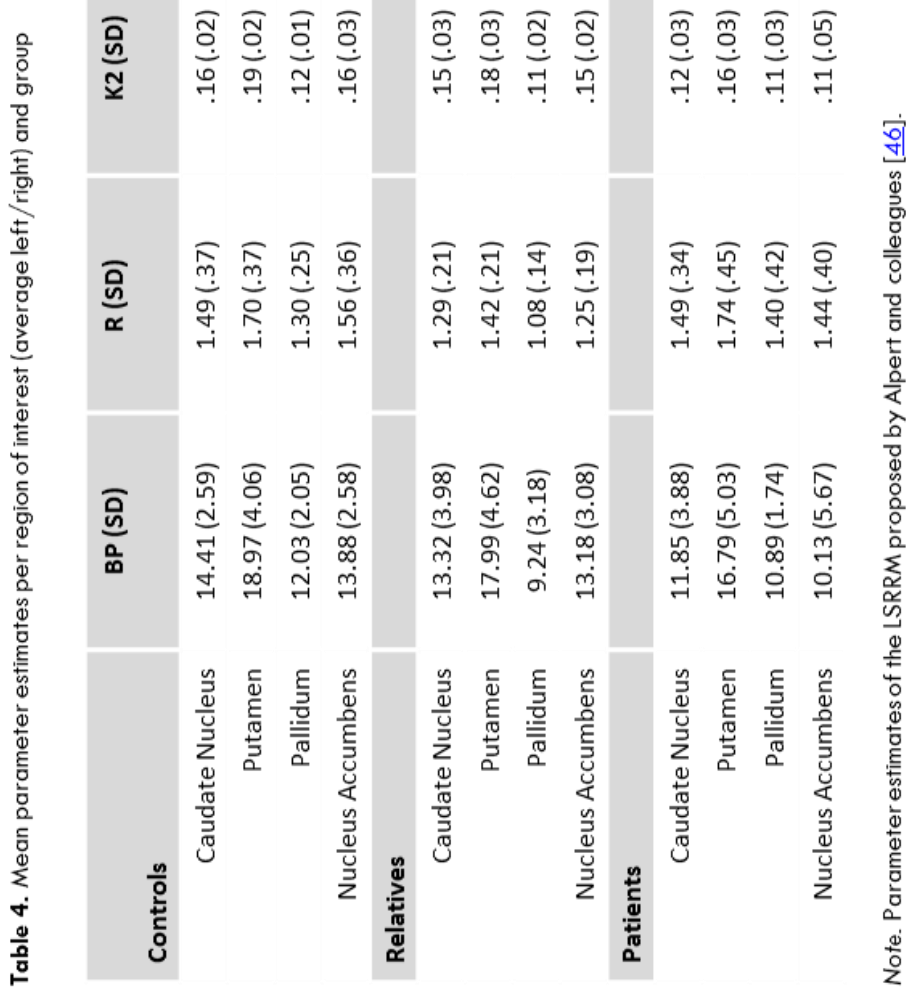




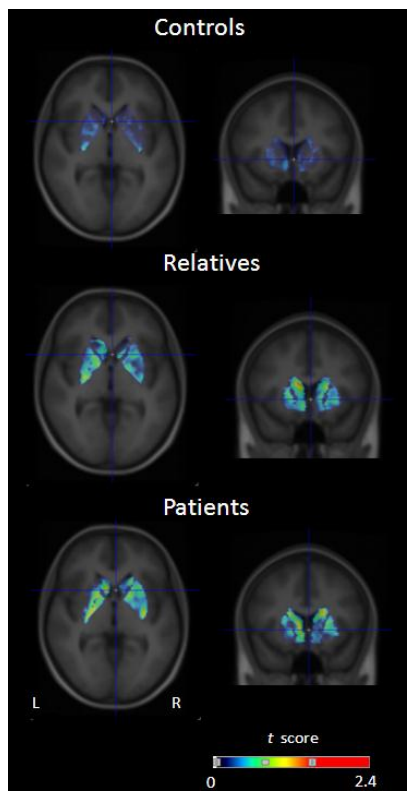

Figure 4. Mean statistical parametric $t$ map of $\gamma$ in sagittal (left) and coronal (right) sections overlaid on a MRI template, showing THC-induced $\left[{ }^{18} \mathrm{~F}\right]$ fallypride displacement at the level of the striatum $(x=0, y=11, z=-4)$ for controls (top row), relatives (middle row) and patients (bottom row).
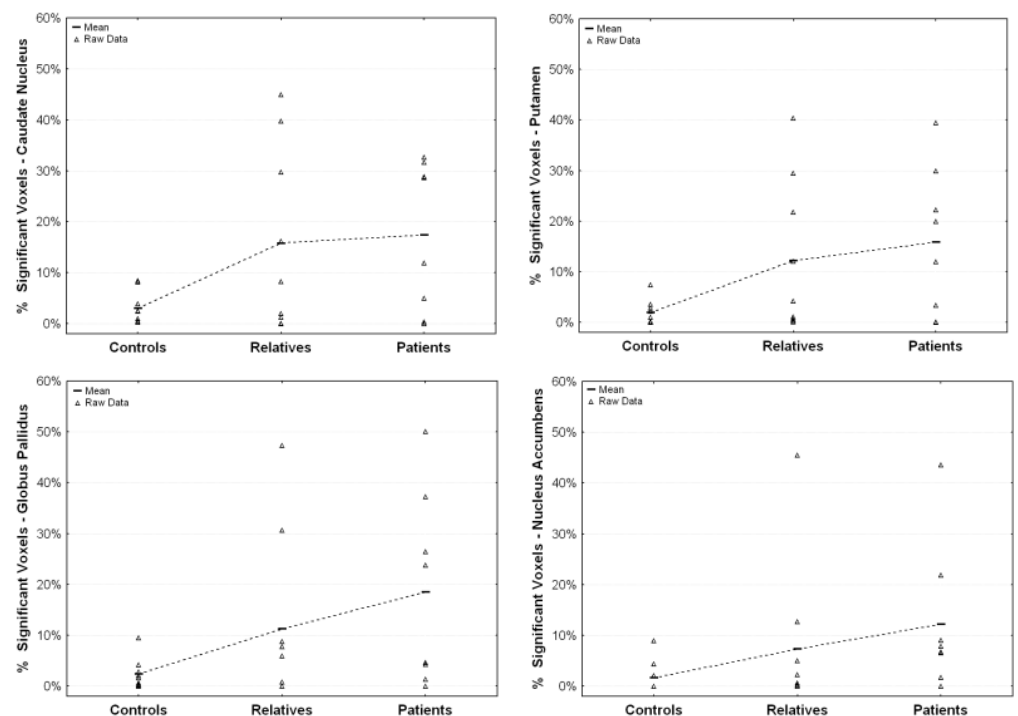

Figure 5. Percentage of voxels with significant THC-induced dopamine release in the caudate nucleus, putamen (top row), globus pallidus and nucleus accumbens (bottom row) for controls, relatives and patients. Horizontal lines indicate the mean value for each group 


\section{Discussion}

The present study revealed the novel finding of striatal DA release following pulmonary administration of THC in a group of patients with psychotic disorder and unaffected relatives. Consistent with most previous work, no DA release was detected in healthy control participants.

\section{THC-induced dopamine release: the mechanism behind cannabis-induced psychosis?}

Numerous animal studies suggest that exogenous cannabinoids such as THC stimulate burst firing of midbrain DA neurons and, as a consequence, facilitate striatal DA release through activation of cannabinoid 1 (CB1) receptors [15, 22, 52-56]. In humans however, evidence that DA may mediate acute effects of THC is scarce, and whether or not DA mediates in part the psychotogenic effects of cannabis remains unclear [6]. The results of three imaging studies investigating THC-induced dopamine release in the human striatum are inconsistent. While Bossong and colleagues [24] report a small, but significant increase in striatal DA, more recent work by Stokes and colleagues [25] as well as Barkus and colleagues [26] did not observe such an effect. Our present study agrees with the most recent studies, as no THC-induced DA release was detected in healthy controls. However, in both patients with psychotic disorder and unaffected relatives, pulmonary administration of THC was associated with a subsequent increase in striatal DA. This is in line with epidemiological and experimental work, demonstrating that patients with a psychotic disorder as well as individuals at risk for psychosis show increased vulnerability to the psychosis-inducing effects of cannabis at the behavioral level $[28,30,31,57,58]$. Thus, our findings suggest that in individuals at risk for psychotic disorder, increased sensitivity to the effects of cannabis may be mediated by striatal dopamine. Notably, in both patients and relative, dopamine release was most pronounced in the caudate nucleus, and dopaminergic hyperactivity in this region is thought to play an important role in the pathophysiology of psychotic symptoms [59]. The apparent discrepancy in the findings presented by Bossong and colleagues [24] may thus be explained by sample admixture of individuals with higher than average liability for psychotic disorder.

The present findings fit with animal work demonstrating interaction between the endocannabinoid and the DA system, in particular with regard to regulation of mesolimbic DA transmission [56, 60]. However, it has also been shown that part of the signaling activity mediated by the endocannabinoid system actually takes place downstream of DA neurotransmission in terms of D2 receptor activation [61, 62], and DA may conversely regulate endocannabinoid function [63]. In line with this, acute psychosis, which is thought to be characterized by dopaminergic hyperactivity in 
striatal brain regions, has been associated with increased levels of the endocannabinoid anandamide in cerebrospinal fluid (CSF), independent of cannabis use and inversely correlated with psychotic symptoms [64, 65]. In addition, patients treated with D2 receptor blocking agents had lower CSF levels of anandamide [65]. Moreover, elevated levels of anandamide may be present in patients in the prodromal phase of psychotic disorder [66]. Research has additionally indicated that the density of $C B 1$ receptors may be altered in individuals with psychotic disorder [67, 68]. Together, these observations suggest an important role of the endocannabinoid system in the pathophysiology of schizophrenia and may furthermore explain our finding that exogenous cannabinoids such as THC affect DA neurotransmission particularly in individuals at risk for DA dysregulation, such as patients with psychotic disorder and first degree relatives.

Taken together, the results of the present study are in line with earlier studies demonstrating that THC does not significantly increase striatal DA in healthy volunteers. However, the novel finding that in patients with psychotic disorder as well as in their first-degree relatives, THC was associated with increased DA release in striatum, most markedly in the caudate nucleus, may explain previous discrepancies. DA thus may mediate in part the psychosis-inducing effects of cannabis, conditional on additional risk of DA dysregulation.

\section{Limitations}

The present findings have to be interpreted in the light of some limitations. The use of the LSRRM has several practical advantages, such as the requirement for only a single radiochemical synthesis and administration and avoidance of session effects. However, practical implementation of the model implies that time-dependent alterations in regional cerebral blood flow ( $\mathrm{rCBF}$ ) are not fully accounted for. Still, as argued by Christian and colleagues [34], using a single injection protocol in combination with the in vivo kinetics of $\left[{ }^{18} \mathrm{~F}\right]$ fallypride may minimize the possible confounds of changing rCBF associated with drug administration. Second, due to the constraint of a one-day protocol, the order of drug administration was only singleblind, so that drugs were not administered randomly, but in the same order for every subject. However, since individuals were told that the order of administration would occur randomly, and the majority of individuals was not able to accurately indicate the active drug when asked at the end of the experiment expectation bias seems unlikely, and would not explain differential effects across the three groups. Third, maximum levels of THC were relatively low in the present study compared to studies using the same amount of THC under the same administration protocol [24, 39]. From these studies, it is known that THC reaches a peak concentration in plasma at 5 minutes after administration and decreases rapidly thereafter. In our study, due to practical reasons and despite major effort, blood samples were not always taken 
at exactly 5 minutes after THC inhalation. Therefore, the relatively low THC plasma concentrations in the present study are likely due to the fact that the maximum peak was missed in some individuals. Subtle differences in the timing of blood sampling might also explain the relatively high between-subject variability. Also, since our sample included frequent cannabis users, of whom the majority used daily, it is not surprising that urinalysis was positive for 18 participants, which equals approximately $70 \%$ of the sample. Therefore, compliance with the study protocol (i.e. abstinence from cannabis during the 5 days prior to scanning) could only be confirmed by interview. Finally, the present finding of a group difference in ligand displacement in several subregions of the striatum has to be interpreted in light of rather low power (0.6). Replication in a larger group is therefore imperative. 


\section{References}

1. Moore, T.H., et al., Cannabis use and risk of psychotic or affective mental health outcomes: a systematic review. Lancet, 2007. 370(9584): p. 319-28.

2. Zammit, S., et al., Effects of cannabis use on outcomes of psychotic disorders: systematic review. British Journal of Psychiatry, 2008. 193(5): p. 357-63.

3. Henquet, C., et al., The environment and schizophrenia: the role of cannabis use. Schizophrenia Bulletin, 2005. 31(3): p. 608-12.

4. Murray, R.M., et al., Cannabis, the mind and society: the hash realities. Nature Reviews Neuroscience, 2007. 8(1 1): p. 885-95.

5. Henquet, C., et al., Gene-environment interplay between cannabis and psychosis. Schizophrenia Bulletin, 2008. 34(6): p. $1111-21$.

6. Kuepper, R., et al., Does dopamine mediate the psychosis-inducing effects of cannabis? A review and integration of findings across disciplines. Schizophr Res, 2010. 121 (1-3): p. 107-17.

7. Arnone, D., et al., Corpus callosum damage in heavy marijuana use: preliminary evidence from diffusion tensor tractography and tract-based spatial statistics. Neuroimage, 2008. 41(3): p. 1067 74.

8. Matochik, J.A., et al., Altered brain tissue composition in heavy marijuana users. Drug Alcohol Depend, 2005. 77(1): p. 23-30.

9. Wilson, W., et al., Brain morphological changes and early marijuana use: a magnetic resonance and positron emission tomography study. J Addict Dis, 2000. 19(1): p. 1-22.

10. Yucel, M., et al., Regional brain abnormalities associated with long-term heavy cannabis use. Arch Gen Psychiatry, 2008. 65(6): p. 694-701.

11. DeLisi, L.E., The effect of cannabis on the brain: can it cause brain anomalies that lead to increased risk for schizophrenia? Curr Opin Psychiatry, 2008. 21 (2): p. 140-50.

12. Linszen, D. and T. van Amelsvoort, Cannabis and psychosis: an update on course and biological plausible mechanisms. Current Opinion in Psychiatry, 2007. 20(2): p. 116-20.

13. Kano, M., et al., Endocannabinoid-mediated control of synaptic transmission. Physiol Rev, 2009. 89(1): p. 309-80.

14. Pertwee, R.G., The diverse $C B 1$ and $C B 2$ receptor pharmacology of three plant cannabinoids: delta9tetrahydrocannabinol, cannabidiol and delta9-tetrahydrocannabivarin. Br J Pharmacol, 2008. 153(2): p. $199-215$.

15. Riegel, A.C. and C.R. Lupica, Independent presynaptic and postsynaptic mechanisms regulate endocannabinoid signaling at multiple synapses in the ventral tegmental area. J Neurosci, 2004. 24(49): p. $11070-8$

16. Chevaleyre, V., K.A. Takahashi, and P.E. Castillo, Endocannabinoid-mediated synaptic plasticity in the CNS. Annu Rev Neurosci, 2006. 29: p. 37-76.

17. Howes, O.D. and S. Kapur, The dopamine hypothesis of schizophrenia: version III--the final common pathway. Schizophrenia Bulletin, 2009. 35(3): p. 549-62.

18. Kapur, S., How antipsychotics become anti-'psychotic' - from dopamine to salience to psychosis, Trends in Pharmacological Sciences, 2004. 25: p. 402-406.

19. Chen, J., et al., Delta 9-tetrahydrocannabinol enhances presynaptic dopamine efflux in medial prefrontal cortex. Eur J Pharmacol, 1990. 190(1-2): p. 259-62.

20. Pistis, M., et al., Delta(9)-tetrahydrocannabinol decreases extracellular GABA and increases extracellular glutamate and dopamine levels in the rat prefrontal cortex: an in vivo microdialysis study. Brain Res, 2002. 948(1-2): p. 155-8.

21. Jentsch, J.D., et al., Delta 9-tetrahydrocannabinol increases prefrontal cortical catecholaminergic utilization and impairs spatial working memory in the rat: blockade of dopaminergic effects with HA966. Neuropsychopharmacology, 1997. 16(6): p. 426-32. 
22. Tanda, G., F.E. Pontieri, and G. Di Chiara, Cannabinoid and heroin activation of mesolimbic dopamine transmission by a common mul opioid receptor mechanism. Science, 1997. 276(5321): p. 2048-50.

23. Voruganti, L.N.P., et al., Cannabis induced dopamine release: an in-vivo SPECT study. Psychiatry Research: Neuroimaging 2001. 107: p. 173-177.

24. Bossong, M.G., et al., Delta9-Tetrahydrocannabinol Induces Dopamine Release in the Human Striatum. Neuropsychopharmacology, 2008.

25. Stokes, P.R., et al., Can recreational doses of THC produce significant dopamine release in the human striatum? Neuroimage, 2009. 48(1): p. 186-90.

26. Barkus, E., et al., Does intravenous \{triangleup\}9-tetrahydrocannabinol increase dopamine release? A SPET study. J Psychopharmacol, 2010.

27. Lataster, J., et al., Psychosocial stress is associated with in vivo dopamine release in human ventromedial prefrontal cortex: a positron emission tomography study using [(1)F]fallypride. Neuroimage, $2011.58(4):$ p. $1081-9$.

28. D'Souza, D.C., et al., Delta-9-tetrahydrocannabinol effects in schizophrenia: implications for cognition psychosis, and addiction. Biological Psychiatry, 2005. 57(6): p. 594-608.

29. Henquet, $\mathrm{C}$., et al., Psychosis reactivity to cannabis use in daily life: an experience sampling study. $\mathrm{Br} \mathrm{J}$ Psychiatry, 2010. 196(6): p. 447-53.

30. G.R.O.U.P., Evidence that familial liability for psychosis is expressed as differential sensitivity to cannabis: an analysis of patient-sibling and sibling-control pairs. Archives of General Psychiatry, 2011 . 68(2): p. 138-47.

31. van Winkel, R., Family-Based Analysis of Genetic Variation Underlying Psychosis-Inducing Effects of Cannabis: Sibling Analysis and Proband Follow-up. Archives of General Psychiatry, 201 1. 68(2): p. $148-57$.

32. Wechsler, D., WAIS-III Nederlandstalige bewerking. Afnames en scoringshandleiding 2000, Lisse: Swets Test Publishers.

33. American Psychiatric Association, Diagnostic and statistical manual of mental disorders. 4 ed2000, Washington, D.C.: Author.

34. Christian, B.T., et al., Measuring dopamine neuromodulation in the thalamus: using [F-18]fallypride PET to study dopamine release during a spatial attention task. Neuroimage, 2006. 31(1): p. 139-52.

35. McGuffin, P., A. Farmer, and I. Harvey, A polydiagnostic application of operational criteria in studies of psychotic illness. Development and reliability of the OPCRIT system. Archives of General Psychiatry, 1991. 48(8): p. 764-70.

36. Kay, S.R., L.A. Opler, and J.P. Lindenmayer, The Positive and Negative Syndrome Scale (PANSS): rationale and standardisation. British Journal of Psychiatry, 1989. 155(suppl.7): p. 59-67.

37. Robins, L.N., et al., The Composite International Diagnostic Interview. An epidemiologic Instrument suitable for use in conjunction with different diagnostic systems and in different cultures. Archives of General Psychiatry, 1988. 45(12): p. 1069-77.

38. Spitzer, R.L., et al., The Structured Clinical Interview for DSM-III-R (SCID). I: History, rationale, and description. Arch Gen Psychiatry, 1992. 49(8): p. 624-9.

39. Zuurman, L., et al., Effect of intrapulmonary tetrahydrocannabinol administration in humans. J Psychopharmacol, 2008.

40. Hazekamp, A., et al., Evaluation of a vaporizing device (Volcano) for the pulmonary administration of tetrahydrocannabinol. J Pharm Sci, 2006. 95(6): p. 1308-17.

41. Abrams, D.I., et al., Vaporization as a smokeless cannabis delivery system: a pilot study. Clinical Pharmacology and Therapeutics, 2007. 82(5): p. 572-8.

42. Strougo, A., et al., Modelling of the concentration-effect relationship of THC on central nervous system parameters and heart rate - insight into its mechanisms of action and a tool for clinical research and development of cannabinoids. J Psychopharmacol, 2008. 
43. Bowdle, T.A., et al., Psychedelic effects of ketamine in healthy volunteers: relationship to steady-state plasma concentrations. Anesthesiology, 1998. 88(1): p. 82-8.

44. Mukherjee, J., et al., Brain imaging of $18 \mathrm{~F}$-fallypride in normal volunteers: blood analysis, distribution, test-retest studies, and preliminary assessment of sensitivity to aging effects on dopamine $D-2 / D-3$ receptors. Synapse, 2002. 46(3): p. 170-88.

45. Mukherjee, J., et al., Preliminary assessment of extrastriatal dopamine D-2 receptor binding in the rodent and nonhuman primate brains using the high affinity radioligand, $18 \mathrm{~F}$-fallypride. Nucl Med Biol, 1999. 26(5): p. 519-27.

46. Alpert, N.M., et al., A novel method for noninvasive detection of neuromodulatory changes in specific neurotransmitter systems. Neuroimage, 2003. 19(3): p. 1049-60.

47. Badgaiyan, R.D., A.J. Fischman, and N.M. Alpert, Dopamine release during human emotional processing. Neuroimage, 2009. 47(4): p. 2041-5.

48. Lammertsma, A.A. and S.P. Hume, Simplified reference tissue model for PET receptor studies. Neuroimage, 1996. 4(3 Pt 1): p. 153-8.

49. Talairach, J. and P. Tournoux, Co-planar Stereotaxic Atlas of the Human Brain1988, New York: Thieme.

50. Benjamini, Y. and Y. Hochberg, Controlling the false discovery rate: a practical an powerful apporach to multiple testing. Journal of the Royal Statistical Society Series B: Statistical Methodology, 1995. 57: p. 289-300.

51. Genovese, C.R., N.A. Lazar, and T. Nichols, Thresholding of statistical maps in functional neuroimaging using the false discovery rate. Neuroimage, 2002. 15(4): p. 870-8.

52. Sakurai-Yamashita, Y., et al., Delta 9-tetrahydrocannabinol facilitates striatal dopaminergic transmission. Pharmacol Biochem Behav, 1989. 33(2): p. 397-400.

53. Cheer, J.F., et al., Cannabinoids enhance subsecond dopamine release in the nucleus accumbens of awake rats. Journal of Neuroscience, 2004. 24(18): p. 4393-400.

54. Wu, X. and E.D. French, Effects of chronic delta9-tetrahydrocannabinol on rat midbrain dopamine neurons: an electrophysiological assessment. Neuropharmacology, 2000. 39(3): p. 391-8.

55. French, E.D., K. Dillon, and X. Wu, Cannabinoids excite dopamine neurons in the ventral tegmentum and substantia nigra. Neuroreport, 1997. 8(3): p. 649-52.

56. Gessa, G.L., et al., Cannabinoids activate mesolimbic dopamine neurons by an action on cannabinoid CB 1 receptors. Eur J Pharmacol, 1998. 341(1): p. 39-44.

57. Henquet, C., et al., Prospective cohort study of cannabis use, predisposition for psychosis, and psychotic symptoms in young people. British Medical Journal, 2005. 330(7481): p. 11.

58. Henquet, C., et al., An experimental study of catechol-o-methyltransferase Val158Met moderation of delta-9-tetrahydrocannabinol-induced effects on psychosis and cognition. Neuropsychopharmacology, 2006. 31(12): p. 2748-57.

59. Kuepper, R., M. Skinbjerg, and A. Abi-Dargham, The dopamine dysfunction in psychosis revisited: New insights into topography and course, in Handbook of Experimental Pharmacology, Current Antipsychotics, M.A. Geyer and G. Gross, Editors. in press, Springer.

60. Diana, M., et al., Mesolimbic dopaminergic decline after cannabinoid withdrawal. Proc Natl Acad Sci U S A, 1998. 95(17): p. 10269-73.

61. Giuffrida, A., et al., Dopamine activation of endogenous cannabinoid signaling in dorsal striatum. Nature Neuroscience, 1999. 2(4): p. 358-63.

62. Morrison, P.D. and R.M. Murray, From real-world events to psychosis: the emerging neuropharmacology of delusions. Schizophrenia Bulletin, 2009. 35(4): p. 668-74.

63. Leweke, M. and D. Koethe, Cannabis and psychiatric disorders: it is not only addiction. Addiction Biology, 2008. 13(2): p. 264-75.

64. Leweke, M., et al., Elevated endogenous cannabinoids in schizophrenia. Neuroreport, 1999. 10(8): p. 1665-9. 
65. Giuffrida, A., et al., Cerebrospinal anandamide levels are elevated in acute schizophrenia and are inversely correlated with psychotic symptoms. Neuropsychopharmacology, 2004. 29(11): p. 2108-14.

66. Koethe, D., et al., Anandamide elevation in cerebrospinal fluid in initial prodromal states of psychosis. Br J Psychiatry, 2009. 194(4): p. 371-2.

67. Koethe, D., et al., Expression of $C B 1$ cannabinoid receptor in the anterior cingulate cortex in schizophrenia, bipolar disorder, and major depression. J Neural Transm, 2007. 114(8): p. 1055-63.

68. Wong, D.F., et al., Quantification of cerebral cannabinoid receptors subtype 1 (CB1) in healthy subjects and schizophrenia by the novel PET radioligand [1 1C]OMAR. Neuroimage, 2010. 52(4): p. 1505-13. 



\title{
IS PSYCHOTIC DISORDER ASSOCIATED WITH INCREASED
}

\section{LEVELS OF CRAVING FOR CANNABIS? AN EXPERIENCE SAMPLING STUDY}

\author{
Rebecca Kuepper $^{1 *}$, Margreet Oorschot ${ }^{1 *}$, Inez Myin-Germeys ${ }^{1,3}$, \\ Jim van Os ${ }^{1,4}$, Maurice Smits ${ }^{5}$ \& Cécile Henquet ${ }^{1,2}$
}

[*contributed equally]

1 Department of Psychiatry and Neuropsychology, South Limburg Mental Health Research and Teaching Network, EURON, Maastricht University Medical Center, PO Box 616 (location Vijverdal), NL-6200 MD, Maastricht, The Netherlands 2 PsyQ Heerlen, Mondriaan, South Limburg, The Netherlands ${ }^{3}$ School of Psychological Sciences, University of Manchester, UK ${ }^{4}$ King's College London, King's Health Partners, Department of Psychosis Studies, Institute of Psychiatry, London, UK

${ }^{5}$ Mondriaan Zorggroep, South Limburg, The Netherlands submitted 


\begin{abstract}
The prevalence of cannabis use among individuals with psychotic disorder is considerable. However, little is known about patterns of use and factors contributing to continuation of use in this vulnerable population. The present study therefore investigated the role of craving, a central feature of drug-using behavior, in relation to cannabis use in patients with psychotic disorder and healthy controls. Craving was assessed cross-sectionally with the Obsessive Compulsive Drug Use Scale (OCDUS) for cannabis, as well as in the flow of daily life using the Experience Sampling Method (ESM - a structured diary method assessing individuals in daily life). Patients scored higher on the OCDUS than controls, but did not differ from controls in ESM indices of craving in daily life. Nevertheless, the ESM measure of craving better predicted daily life cannabis use and this association was stronger in controls. Craving was not moderated by antipsychotic medication in the patient group. The results indicate that the association between craving and cannabis use is assessed most accurately when assessed in the moment in daily life.
\end{abstract}




\section{Introduction}

The use of cannabis is common in users of mental health services, particularly in patients with psychotic disorder [1, 2] in whom cannabis use has been associated with poor treatment concordance and higher relapse rates [3]. Although cannabis use thus constitutes a major concern in the treatment of patients with psychotic disorder [4], little is known about patterns and mechanisms of continuation of use in this vulnerable population, particularly in association with craving for cannabis.

Craving constitutes a central feature of drug-using behavior and addiction. It is assumed that craving develops as a result of associative learning processes, in which the repeated use of addictive drugs is reinforced by the attribution of (aberrant) incentive salience to the act of drug taking $[5,6]$. Recently, it has been suggested that craving for cannabis is increased in psychosis as patients, independent of frequency of use, experienced significantly more craving than cannabis-using healthy controls [7]. Another recent study suggested that in patients with psychotic disorder, craving was associated with antipsychotic treatment [8], which produces blockade at dopamine D2 receptors. Patients who were receiving risperidone experienced significantly more craving compared to patients treated with olanzapine or clozapine. These antipsychotics differ in D2 receptor occupancy rate, D2 receptor dissociation rate and D1/D2 receptor occupancy rate [9], risperidone having a higher D2 occupancy rate, a lower D1/D2 occupancy rate and a lower dissociation rate $[8,9]$. Thus, there is evidence that craving is increased in psychosis, possibly in association with the use of antipsychotic medication. As craving for cannabis may be particularly instrumental for continuation of cannabis use in patients with psychotic disorder and constitutes an important aspect of drug-using behavior, this issue requires further clarification.

In the above mentioned studies, craving for cannabis was measured primarily with the Obsessive Compulsive Drug Use Scale (OCDUS), which was recently validated for cannabis use [7]. It is a 12-item Likert-rated scale based on the Obsessive Compulsive Drinking Scale [10]. However, the use of cross-sectional questionnaires arguably is inaccurate as it does not allow assessment of dynamic, real-life fluctuations in craving and its association with cannabis use, which are important in users of mental health service given the complexity of drug-using behavior in relation to fluctuating psychiatric symptoms. Therefore, the current study used the Experience Sampling Method (ESM), a real-life self-assessment random time sampling technique, to extend existing findings on craving for cannabis in psychosis to the dynamic context of daily life. The following research questions were examined. First, is craving, measured both with the OCDUS and with ESM, increased in patients with psychotic disorder compared to healthy controls? Second, do OCDUS scores 
reflect craving in daily life measured with ESM? And third, are OCDUS and/or ESM craving associated with cannabis use in daily life and does this association differ between groups? In addition, analyses were carried out in order to investigate the influence of antipsychotic medication in the patient group.

\section{Method}

\section{Participants}

The study included 58 patients with a diagnosis of psychotic disorder and 63 healthy controls. Patients were recruited through in- and out-patient mental health service facilities in South Limburg, The Netherlands, in the context of a treatment study on the effectiveness of motivational interviewing for patients with psychotic disorder and co-morbid cannabis abuse. All patients were frequent cannabis users and willing to receive treatment. Controls were recruited using flyers in local 'coffeeshops' (cafes where cannabis is sold and consumed legally) and through word-by-mouth advertising. Inclusion criteria were i) aged between 18 to 55 years, ii) using cannabis minimally three times a week, and iii) patients only: a diagnosis of psychotic disorder according to the Operational Criteria Checklist (OPCRIT) [11], yielding diagnoses according to Research Diagnostic Criteria after running the OPCRIT computer program. Exclusion criteria were i) weekly use of illicit drugs other than cannabis, ii) alcohol use in excess of five units per day, iii) presence of neurological or psychiatric disorder other than psychosis (patients only) and iv) presence of any neurological or psychiatric disorder (confirmed by OPCRIT) and family history of psychosis (controls only).

\section{Instruments}

\section{Clinical measures}

The Positive and Negative Syndrome Scale (PANSS) [12] was used to assess severity of psychotic symptoms in the patient group. The Obsessive Compulsive Drug Use Scale (OCDUS) for cannabis use [8] was used to assess craving for cannabis in both patients and controls. The OCDUS is a 12-item Likert-rated scale, originally described by Anton and colleagues in work relating to alcohol use [10]. A cannabisspecific version of the OCDUS was recently validated by Dekker and colleagues [7] and successfully applied by Machielsen and colleagues [8], confirming the existence of three subscales which can be best described as 'craving/urge', 'resistance', and 'impact'. In order to capture levels of symptomatology and craving during the ESM week, both scales were administered after the week in which ESM was carried out. 
The Experience Sampling Method (ESM)

ESM is a pseudo-random self-assessment time sampling technique. Feasibility, validity and reliability of ESM in patients with schizophrenia have been demonstrated previously $[13,14]$. Participants received a digital wrist watch and paper-and-pencil ESM booklet. Twelve times a day on six consecutive days, the watch emitted a beep at random moments in 85-minute time blocks between 07:30 $\mathrm{h}$ and 1:30 h. After each beep, participants were asked to fill in a self-assessment form, collecting reports of affect, craving, severity of symptoms and activity rated on 7-point Likert scales at the moment of the beep. Participants were instructed to complete the form immediately after the beep to minimize memory distortion and to record the time at which they completed their report. In addition to filling in selfassessment forms after each beep, participants were asked to fill in a dayevaluation form at the end of each day before they went to sleep. During a briefing session, the ESM procedure was explained and a practice booklet was completed and discussed with the participant to confirm that the scale format was accurately understood. During the ESM week, participants were contacted by phone in order to ensure that they complied with the instructions. Participants were requested not to use illicit drugs other than cannabis during the ESM week and if they did, to tell the investigator about this use. None of the participants was a regular user of drugs other than cannabis. At each beep, when forms were completed, participants were asked to report the exact time. Reports completed more than 5 minutes before and 15 minutes after the beep were excluded from the analyses, given that previous research has shown that remote answers are less reliable and less valid [15]. In addition, given that previous work has shown that measures of individuals with less than $30 \%$ of completed reports are less reliable, participants with less than 24 valid beeps were similarly excluded from the analyses [15].

\section{ESM measures}

Measures of craving and use of cannabis in the flow of daily life were derived from the ESM reports as described below.

Craving intensity was assessed with the item 'I would like to use cannabis'. This item was rated on a 7-point Likert scale from 'not at all' to 'very much'. The score on this item formed a continuous variable ('ESM craving intensity') referring to the moment of the beep. The mean score per person across all beeps ('mean ESM craving intensity') was used to investigate overall group differences on this item.

Craving frequency was measured by calculating the proportion of beeps per person at which craving was reported (dichotomous, cut-off score of $>2$ on the craving item), relative to the number of valid beeps.

Craving variability was defined as the mean square successive difference (MSSD), which is calculated as the average of the squared difference between successive continuous craving scores. The MSSD measure is suggested by several authors as a 
measure of variability on a certain item across moments in momentary assessment studies and was accordingly adapted to represent variability in craving across measurement moments [16, 17].

Cannabis use was assessed with the item 'Since the previous beep I have used cannabis' and was dichotomously coded yes versus no. This item formed the variable 'momentary cannabis use' (dichotomous, yes versus no) and referred to cannabis use in the period between two beeps (see figure 1). In addition, to obtain a measure of frequency of cannabis use throughout the ESM week, we calculated the percentage of beeps per person at which cannabis use was reported, relative to the number of valid beeps. This score formed the continuous variable 'cannabis use frequency'.

Antipsychotic medication

Conform previous work [8, 18], antipsychotic medication was classified as tight (haloperidol, flupentixol, penfluridol, risperidone) or loose binding (olanzapine) based on published D2 receptor occupancy and dissociation rates.

\section{Statistical analysis}

Data were analyzed using Stata ${ }^{\circledR}$ release 11.0. All analyses were a priori adjusted for age and sex.

Individual-level analyses testing differences between groups

Linear regression models were used to investigate i) overall group differences on OCDUS scores, mean ESM craving intensity, mean ESM craving frequency, mean ESM craving variability and cannabis use frequency, ii) the association between OCDUS scores and mean ESM craving intensity with cannabis use frequency on the one hand, and iii) the association between scores on the OCDUS, including its subscales, and mean ESM craving intensity on the other. Additional analyses were performed in order to investigate the effect of antipsychotic medication in the patient group.

Momentary-level ESM analyses

Due to the multilevel structure of ESM (i.e. multiple assessments clustering within subjects) multilevel random effects regression analysis was applied using the xtgee routine, yielding odds ratio's (OR), to test the association between ESM craving intensity at time point ( $t$ ) (independent variable) and momentary cannabis use reported at time point $(t+1)$ dependent variable (figure 1). The interaction with group was calculated in order to investigate whether this association differed between groups. These analyses were additionally adjusted for cannabis use, reported at time point $(t)$. Analyses were performed in order to investigate the effect of antipsychotic medication in the patient group. 


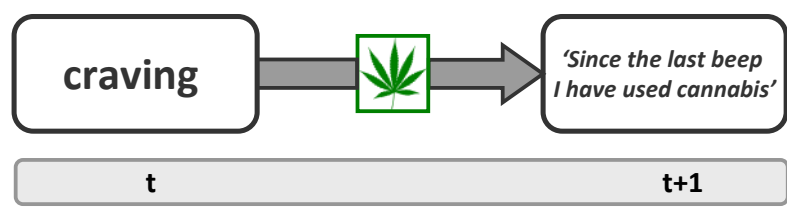

Figure 1. Moment-to-moment analyses of association between ESM craving intensity at time $t$ and momentary cannabis use between $t$ and $t+1$, under additional adjustment for cannabis use reported at time $t$.

\section{Results}

\section{Sample}

Of the 58 patients, $18(31.0 \%)$ had fewer than 24 valid reports and were therefore excluded from the analyses. Of the 63 controls, $6(9.5 \%)$ were excluded due to fewer than 24 valid reports. Thus, the final sample consisted of 40 patients with psychotic disorder (24 male, $60.0 \%$; for OPCRIT diagnoses see table 1) and 57 controls (41 male, $71.9 \%$ ). Eight participants (3 patients and 5 controls) used cannabis in combination with other drugs during a total of $12(0.9 \%)$ of all beeps at which cannabis use was reported $(n=1347)$. To avoid confounding by other drug use, these observations were excluded from the analyses. Patients were receiving either tight binding agents $(N=12)$, loose binding agents $(N=14)$, a combination of antipsychotics $(N=10)$ or no medication $(N=4)$. On average, patients were older, had lower educational level and, per definition, had higher PANSS scores than controls. Furthermore, patients had collected less valid ESM reports than controls. Demographic and clinical characteristics are summarized in table 1.

\section{Individual-level analyses: group differences}

Patients scored significantly higher on the total OCDUS than controls $(B=1.18, p=$ 0.022). Patients scored significantly higher than controls on the OCDUS subscale 'craving/urge' $(B=1.39, p=0.036)$ and on the subscale 'impact' $(B=1.43, p=$ $0.009)$, whereas patients and controls did not differ on the subscale 'resistance' $(B=$ $0.05, p=0.958)$. No differences between patients and controls were apparent with regard to mean $E S M$ craving intensity $(B=0.43, p=0.168$ ) or mean $E S M$ craving frequency $(B=12.67, p=0.062)$. The two groups also did not differ in mean ESM craving variability $(B=2.17, p=0.289)$, and similarly did not differ in mean ESM cannabis use frequency $(B=7.36, p=0.139)$. See table 2 for mean scores per group. 
Both OCDUS total score and mean ESM craving intensity were significantly associated with mean ESM cannabis use frequency (BocDus $=14.05, \mathrm{p}<.001$; $\mathrm{B}_{\mathrm{ES} \text { Mcraving }}=13.49, \mathrm{p}<0.001$ ), and this association did not differ between patients and controls $(p>0.05)$ When OCDUS total score and mean ESM craving intensity were entered in the model simultaneously, only mean ESM craving intensity remained significantly associated with mean ESM cannabis use frequency ( $B_{\text {ESMcraving }}=13.08$, $p<0.001$; BocDus $=-2.11, p=0.619)$.

OCDUS total score was significantly associated with mean ESM craving intensity $(B=$ 4.33, $p<0.001)$. Regarding the OCDUS subscales, significant associations with mean ESM craving intensity were found for 'craving/urge' ( $B=0.48, p<0.001$ ) and 'impact' $(B=0.33, p<0.001)$, but not for 'resistance' $(B=0.11, p=0.223)$.

Analyses did not reveal any differences in scores on the OCDUS, mean ESM craving intensity, mean ESM craving frequency or mean ESM craving variability between patients receiving tight binding agents versus patients receiving loose binding agents or no antipsychotic medication (all $p>0.05$, see table 3 for mean scores per medication group).

\section{Momentary-level ESM analyses}

Momentary ESM craving intensity was significantly associated with subsequent momentary cannabis use $(O R=1.49, \mathrm{p}<0.001)$, and this association was somewhat stronger in controls as compared to patients (Fig. 2, $p=0.001$ ). The association between ESM craving intensity and momentary cannabis use was not moderated by medication status in the patient group (all $p>0.05$ ).

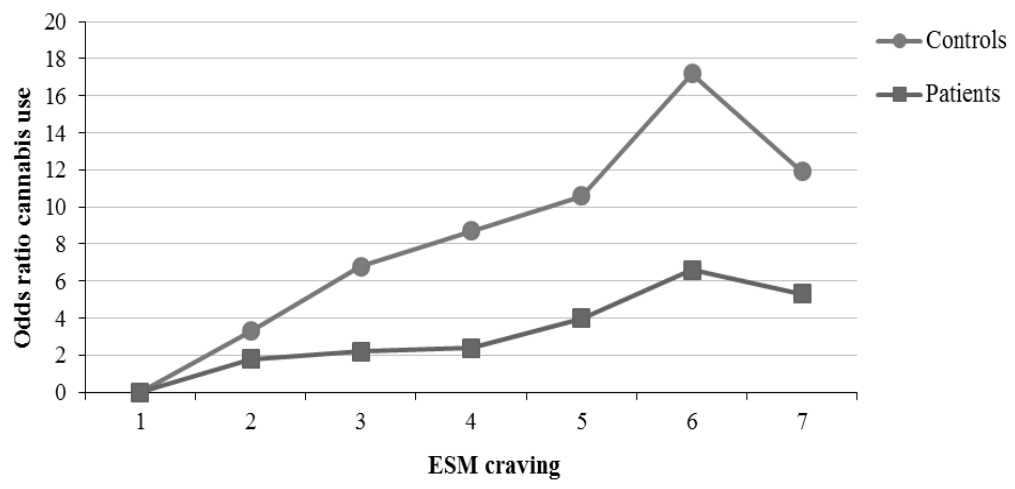

Figure 2. Moment-to-moment association between ESM craving intensity at time ( $t$ ) (ranging from $1=$ not at all to $7=$ very much) and momentary cannabis use (yes versus no) between $t$ and $(t+1)$. 
Table 1. Demographic and clinical characteristics

\begin{tabular}{|c|c|c|c|}
\hline & Patients & Controls & Patients vs. Controls \\
\hline $\mathrm{N}$ & 40 & 57 & n.a. \\
\hline Mean age (SD) & $37.7(9.15)$ & $27.4(9.16)$ & $F(1,95)=28.22, p<0.001$ \\
\hline Male \% (n) & $61.5(24)$ & $71.9(41)$ & $\chi^{2}(1)=1.14, p=0.285$ \\
\hline \multicolumn{4}{|l|}{ Education $\%(n)^{*}$} \\
\hline Elementary & $27.8(10)$ & $14.3(8)$ & \multirow{3}{*}{$\chi^{2}(2)=11.6, p=0.003$} \\
\hline Secondary & $61.1(22)$ & $41.1(23)$ & \\
\hline Higher & $11.1(4)$ & $44.6(25)$ & \\
\hline \multicolumn{4}{|l|}{ Mean PANSS scores (SD) } \\
\hline Positive symptoms & $1.73(0.77)$ & $1.05(0.10)$ & $F(1,91)=42.41, p<0.001$ \\
\hline Negative symptoms & $1.58(0.61)$ & $1.22(0.23)$ & $F(1,89)=25.61, p<0.001$ \\
\hline Global symptoms & $1.53(0.44)$ & $1.10(0.16)$ & $F(1,86)=45.81, p<0.001$ \\
\hline \multicolumn{4}{|l|}{ OPCRIT diagnoses \% (n) } \\
\hline Schizophrenia & $40.0(16)$ & n.a. & n.a. \\
\hline Schizoaffective disorder & $5.0(2)$ & n.a. & n.a. \\
\hline Other psychoses & $55.0(22)$ & n.a. & n.a. \\
\hline Current use of medication $\%(n)$ & $90.0(36)$ & $0.00(0)$ & n.a. \\
\hline Tight binding antipsychotics & $33.3(12)$ & n.a. & n.a. \\
\hline Loose binding antipsychotics & $38.9(14)$ & n.a. & n.a. \\
\hline Other/combination & $27.8(10)$ & n.a. & n.a. \\
\hline Mean valid ESM reports $\%(n)$ & $44.4(32)$ & $56.9(41)$ & $F(1,95)=14.99, p=0.002$ \\
\hline Cannabis use frequency $\%(n)$ & $40.6(13)$ & $36.6(15)$ & $F(1,93)=0.47, p=0.496$ \\
\hline
\end{tabular}

Note. Some percentages do not total 100 due to rounding.

* Information is missing in $n=1$ control subject and $n=4$ patients.

Table 2. Mean ESM and OCDUS scores per group.

\begin{tabular}{|c|c|c|}
\hline & Patients & Controls \\
\hline Mean ESM craving intensity (SD) & $3.57(1.31)$ & $3.20(1.26)$ \\
\hline ESM craving frequency $\%$ (SD) & $61.43(29.20)$ & $52.71(27.82)$ \\
\hline ESM craving variability MSSD (SD) & $15.58(8.69)$ & $13.90(9.14)$ \\
\hline \multicolumn{3}{|l|}{ Mean OCDUS scores (SD) } \\
\hline Craving/urge & $3.08(1.02)$ & $2.45(0.69)$ \\
\hline Resistance & $3.21(1.02)$ & $3.20(1.21)$ \\
\hline Impact & $2.27(0.80)$ & $1.65(0.63)$ \\
\hline Total score & $2.76(0.80)$ & $2.24(0.55)$ \\
\hline
\end{tabular}


Table 3. Mean scores per medication group (patients only; all $p>0.05$ ).

\begin{tabular}{|l|c|c|c|}
\hline & $\begin{array}{c}\text { Tight } \\
(\mathbf{N}=12)\end{array}$ & $\begin{array}{c}\text { Loose } \\
(\mathbf{N}=14)\end{array}$ & $\begin{array}{c}\text { No medication } \\
(\mathbf{N}=\mathbf{4})\end{array}$ \\
\hline $\begin{array}{r}\text { Mean ESM craving intensity (SD) } \\
\text { ESM craving frequency \% (SD) }\end{array}$ & $\begin{array}{c}\text { 3.27 (1.63) } \\
\text { ESM craving variability MSSD (SD) }\end{array}$ & $3.83(1.14)$ & $2.92(0.73)$ \\
\hline Mean OCDUS scores (SD) & $14.04(11.56)$ & $16.70(6.90)$ & $11.62(4.30)$ \\
\hline Craving/urge & $3.07(1.41)$ & $3.38(1.01)$ & $2.65(0.19)$ \\
Resistance & $3.05(1.33)$ & $3.14(1.19)$ & $3.38(0.75)$ \\
Impact & $2.29(1.13)$ & $2.51(0.59)$ & $2.05(0.93)$ \\
Total score & $2.74(1.10)$ & $2.98(0.74)$ & $2.52(0.41)$ \\
\hline
\end{tabular}

Note. Classification of medication according to high or loose binding characteristics at D2 receptors was done in agreement with Lataster and colleagues [18].

\section{Discussion}

The present study demonstrated that craving is increased in patients with psychotic disorder, when measured with the OCDUS. Yet, in daily life, patients and controls did not differ with regard to craving intensity, craving variability or craving frequency. In patients and controls, both OCDUS craving and ESM craving intensity were associated with frequency of cannabis use. However, ESM craving appeared to be a better predictor of cannabis use in daily life, given that when both terms were entered in the individual-level prediction model of cannabis use simultaneously, only ESM craving remained significant. Analysis of moment-to-moment assessment of craving in association with cannabis use showed that craving intensity was predictive of subsequent cannabis use. Although sample sizes per medication subgroup were small and individual-level comparisons likely had low power, no differences were revealed between patients receiving tight or loose binding agents or no medication.

\section{Craving for cannabis in daily life}

In line with the findings of Dekker and colleagues [7], increased craving was apparent in patients with psychotic disorder, relative to controls, in a comparison of OCDUS scores. Yet, when craving was measured in the moment with ESM, patients and controls reported comparable levels of craving intensity. One possibility to account for this discrepancy is that our ESM craving measure is not a valid tool for the assessment of craving for cannabis. However, OCDUS craving and ESM craving were significantly associated with each other, with the exception of the OCDUS subscale 'resistance', suggesting that at least part of the concept of craving is reflected by our daily life ESM craving measure. In addition, when both OCDUS 
scores and mean ESM craving intensity were simultaneously entered in the prediction model, only mean ESM craving remained significantly associated with frequency of cannabis use in daily life, suggesting that OCDUS is reducible to the ESM measure in this regard. Together with the finding that momentary ESM craving intensity was significantly associated with subsequent cannabis use, this supports the validity of ESM craving and furthermore indicates that craving as captured by the OCDUS is less predictive for daily life cannabis use. One explanation for this finding is that reporting on cross-sectional questionnaires is subject to interpretation, in comparison with reporting in the moment with ESM. In OCDUS, patients may report their (sometimes erroneous) interpretation of cannabis use as beneficial for symptoms [19], resulting in higher OCDUS craving scores, whereas during ESM individuals report momentary craving without interpretation of the perceived relationship between cannabis use and symptoms.

The association between ESM craving intensity and subsequent cannabis use was stronger for controls than for patients, yet no differences emerged with regard to overall craving intensity, craving frequency and craving variability. Since patients and controls did not differ with regard to the OCDUS subscale 'resistance' it is unlikely that the weaker association in patients with psychotic disorder is due to being more resistant to urges of craving. One explanation may be that patients with psychotic disorder are prevented from use more often than controls, for example due to lack of money or limited access to sites where cannabis may be obtained. Alternatively, cannabis use in patients may occur more independently of inner cues such as craving and may instead be under stronger guidance by other, unmeasured factors, such as fluctuating mental states.

\section{Antipsychotic medication and craving in patients with psychotic disorder}

In contrast to Machielsen and colleagues [8], who showed that patients receiving tight binding agents experienced significantly more craving than patients treated with loose binding antipsychotics, our study did not reveal any significant differences with regard to antipsychotic medication. Moreover, inspection of the mean OCDUS or ESM craving scores suggests that patients treated with tight binding agents experience less craving, both with regard to intensity and frequency, than patients receiving loose binding agents. Medication-free patients, on the other hand, had the lowest mean scores. Yet, none of these differences reached significance in our analyses. One explanation may be lack of power due to small sample sizes, although this would not explain the different directionality of the findings compared to Machielsen and colleagues (2011), and the ESM-level analyses were more powerful than the individual-level analyses. Therefore, the current results may also indicate that craving is increased in psychosis, independently of antipsychotic medication, supporting the later findings by Dekker and colleagues (in press). 


\section{Limitations}

The current results have to be interpreted in the light of several methodological limitations. First, compliance with the research protocol is a crucial element of the ESM research method. Some authors have cast doubt on compliance in paper-andpencil ESM studies and preferred the use of electronic devices [20]. However, two studies in which paper-and-pencil diary and electronic diary data were collected using comparable procedures, suggested good compliance rates with the time protocol and demonstrated that both methods yielded data comparable in terms of both psychometric features and research findings [21, 22]. Second, reports on craving and cannabis use were based on self-report and not confirmed by objective measures, such as urine analysis. However, underreporting of cannabis use is unlikely, as consumption of cannabis is legal in The Netherlands and participants reported cannabis use at $50 \%$ of the beeps. Finally, the current ESM protocol assessed craving with the use of a single item, which may not be sufficient to capture the complex concept of craving, compared to multi-item questionnaires such as the OCDUS. However, the current analyses suggest that our ESM craving measure is a better predictor of daily life cannabis use than the OCDUS, lending support to the validity of the ESM craving item.

\section{Conclusion}

Taken together, and several limitations notwithstanding, the findings suggest that craving is increased in patients with psychosis, independent of antipsychotic medication, when assessed with cross-sectional questionnaires such as the OCDUS that may carry more interpretational judgments than in-the-moment ESM reports. In contrast, daily life craving does not seem to differentiate between patients and controls, yet craving at ESM level is strongly associated with daily life cannabis use, independent of the cross-sectional OCDUS measure. Therefore, the current results emphasize the need to further investigate craving as a factor maintaining cannabis use in patients with psychotic disorder, especially regarding the influence of antipsychotic medication. Deeper insight into patterns of craving in relation to cannabis use in psychosis is crucial for understanding the co-morbidity between psychosis and cannabis use disorders and for developing new and effective treatment programs. 


\section{References}

1. Gregg, L., C. Barrowclough, and G. Haddock, Reasons for increased substance use in psychosis. Clinical Psychology Review, 2007. 27(4): p. 494-510.

2. Green, B., R. Young, and D. Kavanagh, Cannabis use and misuse prevalence among people with psychosis. Br J Psychiatry, 2005. 187: p. 306-13.

3. Zammit, S., et al., Effects of cannabis use on outcomes of psychotic disorders: systematic review. British Journal of Psychiatry, 2008. 193(5): p. 357-63.

4. Barrowclough, C., et al., Integrated motivational interviewing and cognitive behavioural therapy for people with psychosis and comorbid substance misuse: randomised controlled trial. British Medical Journal, 2010. 341 : p. c6325.

5. Franken, I.H., Drug craving and addiction: integrating psychological and neuropsychopharmacological approaches. Prog Neuropsychopharmacol Biol Psychiatry, 2003. 27(4): p. 563-79.

6. Robinson, T.E. and K.C. Berridge, The neural basis of drug craving: an incentive-sensitization theory of addiction. Brain Res Brain Res Rev, 1993. 18(3): p. 247-91.

7. Dekker, N., et al., Craving for cannabis in patients with psychotic disorderd, their non-affected siblings and healthy controls: psychometric analysis of the Obsessive Compulsive Drug Use Scale. International Journal of Methods in Psychiatric Research, in press.

8. Machielsen, M., et al., Differences in craving for cannabis between schizophrenia patients using risperidone, olanzapine or clozapine. J Psychopharmacol, 2011.

9. Seeman, P. and T. Tallerico, Antipsychotic drugs which elicit little or no parkinsonism bind more loosely than dopamine to brain D2 receptors, yet occupy high levels of these receptors. Mol Psychiatry, 1998. 3(2): p. 123-34.

10. Anton, R.F., D.H. Moak, and P. Latham, The Obsessive Compulsive Drinking Scale: a self-rated instrument for the quantification of thoughts about alcohol and drinking behavior. Alcohol Clin Exp Res, 1995. 19(1): p. 92-9.

11. McGuffin, P., A. Farmer, and I. Harvey, A polydiagnostic application of operational criteria in studies of psychotic illness. Development and reliability of the OPCRIT system. Archives of General Psychiatry, 1991. 48(8): p. 764-70.

12. Kay, S.R., L.A. Opler, and J.P. Lindenmayer, The Positive and Negative Syndrome Scale (PANSS): rationale and standardisation. British Journal of Psychiatry, 1989. 155(suppl.7): p. 59-67.

13. Oorschot, M., et al., Momentary assessment research in psychosis. Psychol Assess, 2009. 21(4): p. 498-505.

14. Myin-Germeys, I., et al., Experience sampling research in psychopathology: opening the black box of daily life. Psychol Med, 2009. 39(9): p. 1533-47.

15. Delespaul, P., Empirical studies in ESM reliability and validity, in Assessing schizophrenia in daily life: The Experience Sampling Method, M. de Vries, Editor 1995, Maastricht University Press: Maastricht.

16. Ebner-Priemer, U.W., et al., Analytic strategies for understanding affective (in)stability and other dynamic processes in psychopathology. J Abnorm Psychol, 2009. 11 8(1): p. 195-202.

17. Jahng, S., P.K. Wood, and T.J. Trull, Analysis of affective instability in ecological momentary assessment: Indices using successive difference and group comparison via multilevel modeling. Psychol Methods, 2008. 13(4): p. 354-75.

18. Lataster, J., et al., Emotional experience and estimates of $D(2)$ receptor occupancy in psychotic patients treated with haloperidol, risperidone, or olanzapine: an experience sampling study. J Clin Psychiatry, 2010.

19. Henquet, C., et al., Psychosis reactivity to cannabis use in daily life: an experience sampling study. British Journal of Psychiatry, 2010. 196(6): p. 447-53.

20. Stone, A.A., et al., Patient compliance with paper and electronic diaries. Controlled Clinical Trials, 2003. 24(2): p. 182-99. 
160 | IS CRAVING FOR CANNABIS INCREASED IN PATIENTS WITH PSYCHOTIC DISORDER?

21. Jacobs, N., et al., Electronic monitoring of salivary cortisol sampling compliance in daily life. Life Sciences, 2005. 76(21): p. 2431-43.

22. Green, A.S., et al., Paper or plastic? Data equivalence in paper and electronic diaries. Psychol Methods, 2006. $11(1)$ : p. 87-105. 
\begin{tabular}{l|l} 
CHAPTER 8 & 161
\end{tabular} 



\title{
DOES CANNABIDIOL PROTECT AGAINST THE NEGATIVE EFFECTS OF THC?
}

\author{
Cécile Henquet ${ }^{1,2}$ \& Rebecca Kuepper ${ }^{1}$ \\ 1 Department of Psychiatry and Neuropsychology, South Limburg Mental Health Research and \\ Teaching Network, EURON, Maastricht University Medical Center, PO Box 616 (location \\ Vijverdal), NL-6200 MD, Maastricht, The Netherlands \\ ${ }_{2}^{2}$ PsyQ Heerlen, Mondriaan, South Limburg, The Netherlands
}

British Journal of Psychiatry, 201 1. 197(4): p. 259-260. 


\section{Summary}

A recent study by Morgan and colleagues found that cannabidiol attenuates the acute cognitive effects of delta-9-tetrahydrocannabinol (THC). This is of interest as THC has been associated with the detrimental effects of cannabis on mental health in at-risk users, and the potency of cannabis is increasing across Europe. 


\section{Introduction}

The paper by Morgan and colleagues published in this issue [1] adds to the idea that cannabidiol (a cannabis compound) antagonises the effects of delta-9tetrahydrocannabinol (THC, the main psychoactive compound of cannabis). Cannabidiol itself has no psychoactive properties but instead, as Morgan et al showed, may protect against the cognitive effects of THC. In their naturalistic study, participants consumed their own, self-chosen cannabis and were subsequently tested on a number of cognitive task and questionnaires. Individuals whose self-chosen cannabis contained high levels of cannabidiol showed no memory impairment after consumption of their cannabis, in contrast to the individuals whose self-chosen cannabis contained low levels of cannabidiol: here, cannabis significantly worsened memory performance.

Cannabis is the most frequently used drug in the world and although recent data suggest a stabilisation of cannabis use in most countries in Europe, its use remains particularly popular among young adolescents. That this is of great concern follows from epidemiological studies showing that heavy use during adolescence is particularly detrimental with respect to long-term effects on cognition and mental health. The findings by Morgan and colleagues [1] emphasise the importance of taking into account differences in potency of cannabis preparations. Cannabis potency varies widely between and within countries and between different products. Data from the UK, for instance, show that, on average, cannabis resin contains approximately equal levels of THC and cannabidiol, whereas herbal cannabis contains only moderate levels of THC and almost no cannabidiol. By contrast, sinsemilla, or skunk, contains high levels of THC and almost no cannabidiol [2]. European data show that the use of resin has remained relatively stable over the past years. The use of skunk, however, has significantly increased in the UK and other European countries. It is quite established that the detrimental effects of cannabis on mental health are primarily attributable to THC. In this light, the findings by Morgan and colleagues are relevant as they show that different types of cannabis may moderate the risk for mental health problems to different extents, depending largely on the potency of the cannabis preparation. This is also of relevance for the cannabis-psychosis causality debate and could help to explain why not everyone exposed to cannabis will develop cognitive impairments or psychiatric symptoms. Although a clear dose-response association has consistently been shown between cannabis exposure and psychosis risk, none of the published epidemiological studies differentiated between potency and content of the cannabis they studied. The first evidence that different strains of cannabis may indeed have a differential impact on mental health risk was provided by Di Forti and colleagues [3]. They investigated different types of self-chosen cannabis and found that 
individuals with a first episode of psychosis had used higher-potency cannabis (i.e. skunk) for a longer period of time and with greater frequency than healthy controls [3]. Thus, use of skunk containing high concentrations of THC but low concentrations of cannabidiol was associated with a higher risk of mental health problems.

\section{Does cannabidiol reverse the acute effects of THC?}

In Morgan and colleagues' current study, cannabidiol antagonised the effects of THC on memory [1]. However, no such reversal effects were observed for transient psychotic-like symptoms or anxiety. In a previous study, the authors did show that individuals who smoked cannabis low in cannabidiol reported significantly more psychotic experiences compared with those who smoked cannabis containing high levels of cannabidiol [4]. Although the authors assessed psychosis proneness as opposed to state-related psychotic experiences in that study, the results suggest that cannabidiol may protect against the psychotogenic properties of THC. Experimental studies are in line with this, showing that cannabidiol has the ability to reduce anxiety and psychotomimetic symptoms as well as psychosis-related cognitive distortions induced by $\operatorname{THC}[5,6]$. It remains largely unknown, however, how cannabidiol brings about the THC-antagonising effects, since its pharmacological actions are still elusive. Although cannabidiol seems to have only low affinity for the cannabinoid 1 ( $C B 1$ ) receptor (the main binding site for $T H C$ ), some interaction between cannabidiol and the $C B 1$ receptor has been suggested [7]. Also, cannabidiol may exert its effects by inhibiting reuptake of the endogenous cannabinoid anandamide [7]. In an effort to understand the biological mechanisms underlying the interaction between THC and cannabidiol, Bhattacharyya and colleagues [5] demonstrated opposing effects of THC and cannabidiol on brain activity by using functional magnetic resonance imaging. Delta-9tetrahydrocannabinol attenuated striatal activity and concurrently induced psychotic symptoms, whereas the reverse (i.e. increases in striatal activity) was observed under cannabidiol conditions. Clearly, the molecular actions of cannabidiol are in urgent need of further investigation.

\section{Cannabidiol as a potential antipsychotic drug}

The fact that cannabidiol is able to reduce the acute anxietyinducing and psychotomimetic effects of THC has raised the question whether cannabidiol might also be effective in treating patients with established psychotic disorder [8]. Evidence, although limited, seems promising: Zuardi and colleagues reported improvement in two patients treated with cannabidiol, whose symptoms worsened 
after discontinuation of cannabidiol. In two other patients, cannabidiol had no therapeutic effects; however, these two patients did not respond to other antipsychotic medication either [8]. Preliminary data on a clinical trial including 42 patients described similar positive effects for cannabidiol on Positive and Negative Syndrome Scale scores, but with fewer side-effects than regular treatment with amisulpride [9].

\section{Cannabis and genetic liability}

It seems clear from the above that cannabidiol has the ability to reverse some of the acute effects of THC and may even reduce psychotic symptoms in some patients. Differences in cannabidiol content and THC/cannabidiol ratio between different cannabis preparations may furthermore help to explain why only a minority of cannabis users develop a psychotic illness. Underlying genetic liability for psychosis, however, has been found to determine cannabis sensitivity as well. In a recent study, it was found that individuals at (genetic) risk for psychosis may be more sensitive not only to the psychosis-inducing effects of cannabis in daily life, but also to the positive, mood-enhancing effects of cannabis [10]. Moreover, the rewarding effects of cannabis on mood seem to be acute, whereas psychotic experiences emerge subacute. These findings fit with the idea that different components may have different or even opposing effects, with THC being responsible for the psychosisinducing effects and cannabidiol for the anxiolytic effects. Although numerous studies have shown that cannabis has a negative impact on mental health, especially in individuals with established psychotic disorder, the intriguing question remains as to why patients continue to use these highpotency types of cannabis. Di Forti's data seem to indicate that individuals at increased genetic risk for psychosis (i.e. patients with first-episode psychosis) prefer smoking the higher-potency cannabis. Morgan and colleagues, however, found no evidence that healthy controls with elevated schizotypy scores show preference towards the high THC/low-cannabidiol variants, as the 'high-cannabidiol' and 'low-cannabidiol' groups did not differ with regard to schizotypy [1].

Thus, as different types of cannabis clearly affect mental health differentially, more research is needed to understand how genetic liability may increase sensitivity to and preference for specific types of cannabis. Furthermore, the study by Morgan and colleagues stresses the importance of taking into account differences in cannabis potency when studying acute and long-term effects of cannabis use. Also, when treating patients with psychosis and comorbid cannabis dependence, the type of self-chosen cannabis needs to be considered in order to better understand how not 
168 | DOES CANNABIDIOL PROTECT AGAINST THE NEGATIVE EFFECTS OF THC?

only the rewarding and anxiolytic, but also the psychosis-inducing properties of the drug determine patterns and continuation of use. 


\section{References}

1. Morgan, C.J., et al., Impact of cannabidiol on the acute memory and psychotomimetic effects of smoked cannabis: naturalistic study: naturalistic study [corrected]. British Journal of Psychiatry, 2010. 197(4): p. 285-90.

2. Potter, D.J., P. Clark, and M.B. Brown, Potency of delta 9-THC and other cannabinoids in cannabis in England in 2005: implications for psychoactivity and pharmacology. J Forensic Sci, 2008. 53(1): p. 90-4.

3. Di Forti, M., et al., High-potency cannabis and the risk of psychosis. British Journal of Psychiatry, 2009. 195(6): p. 488-91.

4. Morgan, C.J. and H.V. Curran, Effects of cannabidiol on schizophrenia-like symptoms in people who use cannabis. British Journal of Psychiatry, 2008. 192(4): p. 306-7.

5. Bhattacharyya, S., et al., Opposite effects of delta-9-tetrahydrocannabinol and cannabidiol on human brain function and psychopathology. Neuropsychopharmacology, 2009. 35(3): p. 764-74.

6. Leweke, M., et al., Different effects of nabilone and cannabidiol on binocular depth inversion in Man. Pharmacol Biochem Behav, 2000. 66(1): p. 175-81.

7. Pertwee, R.G., The diverse $C B 1$ and $C B 2$ receptor pharmacology of three plant cannabinoids: delta9tetrahydrocannabinol, cannabidiol and delta9-tetrahydrocannabivarin. Br J Pharmacol, 2008. 153(2): p. 199-215.

8. Zuardi, A.W., et al., Cannabidiol, a Cannabis sativa constituent, as an antipsychotic drug. Braz J Med Biol Res, 2006. 39(4): p. 421-9.

9. Leweke, F., et al., Antipsychotic effects of cannabidiol. European Psychiatry, 2009. 24(suppl 1): p. 207.

10. Henquet, C., et al., Psychosis reactivity to cannabis use in daily life: an experience sampling study. $\mathrm{Br} \mathrm{J}$ Psychiatry, 2010. 196(6): p. 447-53. 



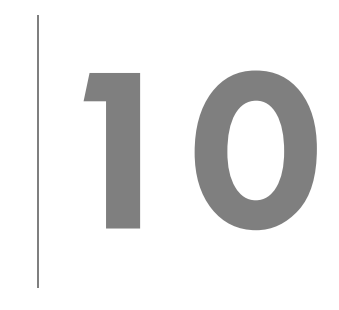

DISCUSSION 
172 | DISCUSSION 


\section{Discussion}

This thesis applied epidemiological, observational and experimental neurochemical imaging techniques to study several aspects of the cannabis-psychosis relationship. First, the temporal association between exposure to cannabis and the incidence of psychotic symptoms was studied. Second, it was investigated whether differential sensitivity to the psychosis-inducing effects of cannabis is influenced by the environment. Third, biological mechanisms that might underlie the association between exposure to cannabis and increased risk for psychosis were elucidated. Finally, in order to increase our understanding of patterns and mechanisms of use in clinical populations, craving for cannabis was investigated as a maintaining factor for continuation of cannabis use in daily life of patients with psychotic disorder, among which cannabis use is particularly prevalent.

Results presented in this thesis show that the use of cannabis preceded the onset of incident, subclinical psychotic symptoms in individuals who had never experienced any psychotic symptoms previously (chapter 2). It was also revealed that continued use of cannabis was associated with an increased risk of persistence of these subclinical psychotic symptoms (chapter 2). Furthermore, it could be demonstrated that individuals who grew up in an urban environment were particularly sensitive to the long-term psychosis-inducing effects of cannabis, providing evidence for environmental moderation of vulnerability to the long-term psychosis-inducing effects of cannabis (chapter 3). Yet, the previously reported link between childhood trauma and increased sensitivity to the effects of cannabis on psychosis risk could not be replicated (chapter 4 ). With regard to possible neurobiological mechanisms of the cannabis-psychosis relationship, it was shown that part of the psychosis-inducing effects of cannabis might be mediated by THC-induced dopamine release in striatal brain regions, in particular in patients with established psychotic disorder and individuals at risk for psychosis (chapters 5, 6 and 7). Finally, it was indicated that patients with established psychotic disorder experience higher levels of craving for cannabis as indicated by higher scores on the Obsessive Compulsive Drug Use Scale (OCDUS) for cannabis. Yet in daily life, craving did not seem to differentiate between patients and controls (chapter 8 ).

\section{Cannabis and psychosis: A causal association?}

Primarily based on the results of numerous epidemiological studies, the link between exposure to cannabis and increased psychosis risk has been well established [1-3]. However, cannabis use is much more prevalent among patients with psychotic disorder compared to the general population [4-6]. Therefore, it has remained a matter of debate, whether the association between cannabis use and the onset of psychosis is indeed causal, or else is due to reverse causality, e.g. in form of self- 
medication, or residual confounding by preexisting symptoms or underlying vulnerability $[7,8]$. This issue was systematically addressed in chapter 2 of this thesis by analyzing data from a prospective population-based cohort study of adolescents and young adults. Due to the long follow-up period, individuals with carefully assessed pre-existing psychotic symptoms could be excluded from the analyses. Still, the study revealed a strong association between cannabis use in the period between baseline and first follow-up and incident psychotic symptoms four years later at second follow-up [9]. Moreover, the presence of psychotic symptoms at baseline did not predict cannabis use at follow-up. This is in accordance with previous research in the same cohort [10] and in contrast to the self-medication hypothesis, which implies that cannabis use is caused by the intention to relieve pre-existing psychotic symptoms or distress resulting from mental illness [11]. The study presented in this thesis thus clarifies the temporal association between exposure to cannabis and the development of psychotic symptoms and, together with the observation that continuation of use is particularly detrimental, provides further evidence for a causal relationship. Of note, in a large Danish cohort study, age of onset of schizophrenia was significantly lower in patients with a history of cannabis use, compared to patients who were never exposed [12]. This link between cannabis use and earlier age of onset of psychotic disorders was recently established by meta-analytic work [13].

Thus, the research presented in this thesis strengthens the evidence that cannabis use is causally linked with increased risk of psychotic disorder [9], the first stage of which is expressed as abnormal persistence of subclinical psychotic symptoms [9, 14]. Alternatively, it has been argued that the link between cannabis and psychosis is due to shared etiology, where similar neuropathology, including alterations in mesolimbic dopamine transmission and dysregulation of cortical, temporal and mesoaccumbens circuits, give rise to both cannabis use and psychotic disorder [15]. However, individuals differ in their sensitivity to the effects of cannabis and not all patients with psychotic disorder have been exposed to cannabis. Hence, cannabis use most likely constitutes a component cause of psychosis, co-depending on other causal influences [16]. Previous research has shown that underlying psychosis liability (as indicated by higher than average scores on scales measuring schizotypy or by familial predisposition) as well as variation in specific genetic polymorphisms may render some individuals more vulnerable to the effects of cannabis than others [10, 17-20]. Little is known however about possible environmental moderation. Therefore, this thesis investigated the influence of two environmental candidates, urbanicity and trauma. Urbanicity is one of the major risk factors of schizophrenia [21] and trauma has been previously suggested to interact with cannabis use in increasing psychosis risk [22-24]. In agreement with the proposed mechanism of environmental moderation, the results described in chapter 3 indicate that the risk of developing 
psychotic symptoms is greater in individuals growing up in an urban environment compared to individuals from rural areas [25]. On the contrary, as discussed in chapter 4 of this thesis, interaction between trauma and cannabis use could not be confirmed [26]. Still, our findings lend support to the idea that in addition to geneenvironment interaction, mechanisms of environment-environment interaction play a causal role in the pathway to psychosis.

\section{Cannabis and psychosis: A role for sensitization?}

As outlined above and discussed in this thesis, cannabis use increases the risk of incident subclinical psychotic symptoms, and moreover, impacts negatively on the persistence of these subclinical symptoms [9]. Previous research has shown that developmental expression of subclinical psychotic experiences in adolescence is mostly transient [27]. Yet, repeated exposure to environmental risk factors, including cannabis use as shown in chapter 2, may cause subclinical psychotic experiences to persist and become more severe, resulting in onset of psychotic illness in a minority of individuals $[9,28,29]$. This is in line with the psychosis-proneness-persistence model, in which genetic predisposition for psychosis synergistically combines with increasing levels of environmental exposure to cause clinical impairment and the onset of psychotic disorder [14]. This has been previously demonstrated for the additive effects of cannabis, trauma and urbanicity [30] and receives further support by our recent finding of interaction between cannabis use and urbanicity. Intriguingly, while cannabis, trauma, and urbanicity do not reflect the same environmental influence, they all impact on the same (psychotic) outcome [31]. This implies a shared interactive pathway. A mechanism of developmental sensitization has been suggested to underlie this pathway, referring to the observation that repeated exposure to a stimulus elicits progressively greater responses over time [32]. Studies demonstrating increasing effects on psychosis risk dependent on cumulative environmental exposure are in agreement with a process of sensitization [9, 33-35] Moreover, the aforementioned synergistic effects between different risk factors, including cannabis use and urbanicity (chapter 3 ), are suggestive of a mechanism of cross-sensitization underlying the developmental pathway to psychosis $[25,30,36]$.

Biologically, the mechanism of sensitization remains understudied and not well understood, at least concerning the evidence in humans. Only one study so far has investigated neurochemical correlates of sensitization to amphetamine in humans and demonstrated alterations in dopaminergic signaling induced by the repeated administration of amphetamine [37]. This is in line with animal studies demonstrating increased activity of dopamine neurons in the VTA [38] and increased dopamine release in the striatum [39-41] following amphetamine sensitization. Yet, as discussed in chapter 6 of this thesis, neurobiological evidence for sensitization 
induced by exposure to cannabis is scarce, although some studies in rats do suggest a dopaminergic mechanism of cannabis sensitization $[42,43]$.

\section{Cannabis and psychosis: Do all roads lead to dopamine?}

While the epidemiological link between cannabis and psychosis has received considerable attention, very little is known about the biological underpinnings. The important role of a dopamine dysfunction in psychosis and schizophrenia summarized in chapter 5 - together with evidence from animal work suggesting interaction between the endocannabinoid and the dopamine system - discussed in chapter 6 - has given rise to the hypothesis that part of the psychosis-inducing effects of cannabis are mediated by dopamine [44]. This hypothesis has been tested recently by groups in the UK and The Netherlands, yet with inconsistent results. While Bossong and colleagues [45] found evidence for dopamine release induced by THC, Stokes and colleagues [46] and Barkus and colleagues [47] did not find such an effect. Notably, these studies were conducted in healthy male subjects who used cannabis only recreationally. However, patients with established psychotic disorder as well as their unaffected relatives have been shown to be more vulnerable to the psychosis-inducing and behavioral effects of cannabis [17, 48]. It is therefore important to investigate the effects of THC on dopamine release in these at-risk populations. Chapter 7 of this thesis presents the first neurochemical imaging study of THC-induced dopamine release in patients with psychotic disorder, unaffected relatives of patients with psychotic disorder and healthy controls. In line with Stokes and colleagues [46] and Barkus and colleagues [47], THC did not induce dopamine release following pulmonary administration of THC in healthy controls. Yet in both patients and relatives, administration of THC was associated with a subsequent increase in striatal dopamine. This suggests that the psychosis-inducing effects of cannabis are mediated by a mechanism of increased striatal dopamine release in individuals at risk for dopamine dysregulation, and thus provides evidence for differential sensitivity towards cannabis on a neurobiological level (chapter 7). However, previously it was demonstrated that pre-treatment with haloperidol, a D2 receptor antagonist, did not prevent the behavioral and psychotomimetic effects produced by administration of THC and even worsened some of the cognitive effects of THC [49]. Although this might corroborate the evidence concerning interaction between the dopaminergic and endocannabinoid system, the findings suggest that the psychosis-inducing and perceptual effects of cannabis involve pathways other than increased dopamine turnover [49]. This would also explain the observation that THC does not induce dopamine release in healthy controls. A recent review on the neuropharmacological mechanisms of endocannabinoid action suggests that, in the striatum, dopaminergic neurotransmission is actually upstream of endocannabinoid function and D2 receptor stimulation leads to endocannabinoid-mediated depression of cortical inputs [50]. Moreover, it appears that drugs which follow this 
mechanism and weaken cortical inputs to medium spiny neurons of the indirect pathway are pro-psychotic [50]. Yet, findings from animal studies indicate that, in the ventral tegmental area, THC might stimulate burst firing of DA neurons and as a consequence increase DA levels in the striatum [44], which is in agreement with the results in chapter 7 , at least concerning patients with psychotic disorder and firstdegree relatives. Thus, the pathway from cannabis to psychosis most likely involves multiple mechanisms and THC might exert its effects by both increasing striatal dopamine and directly influencing synaptic plasticity via a mechanism downstream of dopamine. Still, the former might be particularly true in individuals at risk for psychosis, who are especially prone to dopamine dysregulation (chapter 7).

Notably, early adolescence has been shown to be a particularly vulnerable period for the long-term effects of cannabis, as the risk to develop psychosis later in life is higher for those who started to use cannabis before the age of 15 compared to those who started at a later age $[51,52]$. This is in agreement with preclinical evidence from studies in rats: Chronic treatment with THC led to long-lasting cognitive as well as behavioral changes in both pubertal and adult rats, yet the effects of acute administration were significantly more pronounced in the pubertal rats [53]. Adolescence represents a highly vulnerable period with regard to brain development in general and the development of the endocannabinoid system in particular [54]. Although our understanding of the function and development of the endocannabinoid system is still limited, this system is thought to play a crucial role in the fine-tuning of synaptic transmission, modulating GABAergic, glutamatergic and dopaminergic neurotransmission [55]. Early exposure to exogenous cannabinoids such as THC might accordingly lead to profound disturbances in the development of cortical as well as subcortical neural networks, providing the basis for vulnerability towards mental health problems, including psychosis, later in life [56]. Interestingly, schizophrenia has been associated with functional impairments in the endocannabinoid system, independent of cannabis use. In acutely psychotic patients with schizophrenia as well as in individuals with prodromal schizophrenia pathology, levels of the endogenous cannabinoid anandamide were elevated and inversely correlated with both positive and negative symptoms [57-59]. In addition, studies on the availability of cannabinoid 1 ( $C B 1$ ) receptors, the primary binding site of both endocannabinoids and THC, suggest increased levels of cortical CB 1 receptors in schizophrenia [60-63]. Yet other studies are not consistent with this and found no elevation in $C B 1$ receptor density $[64,65]$. Surprisingly, no study so far has looked at $C B 1$ receptor density in healthy cannabis users. The results of an early rat study are indicative of downregulation of $C B 1$ receptor availability following chronic administration of THC [66]. 
Taken together, the biological pathways from cannabis to psychosis appear to be diverse and research on this topic is still in its infancy. The research presented in this thesis is in line with a role for dopamine in bringing about the psychotomimetic effects of cannabis, at least in individuals at risk for psychosis. Alternatively, studies into the functional role of the endocannabinoid system in both healthy individuals and patients with schizophrenia suggest that (early) exposure to cannabis might induce psychosis risk by disrupting crucial cortical and subcortical neural networks necessary for a well-functioning endocannabinoid system [67]. Further research into the developmental processes and mechanisms of endocannabinoid-mediated neurotransmission in the human brain is therefore warranted.

\section{Cannabidiol as a protective agent}

So far, research on the effects of cannabis in relation to psychosis risk has primarily targeted THC as the main psychoactive ingredient in cannabis. However, apart from THC, cannabis contains a range of other cannabinoids, among others cannabidiol $(C B D)$, and street preparations of cannabis differ largely in their composition [68]. Notably, in contrast to THC, CBD has been shown to have antipsychotic and anxiolytic properties [69] and, as has been suggested among others by Morgan and colleagues [70, 71], might antagonize the effects of THC. Chapter 9 of this thesis provides an editorial on this topic and concludes that it is inevitable to consider differences in the potency (i.e. the amount of THC) of cannabis when studying its effects on behavior, cognition and mental health. Yet so far, only few studies have differentiated between high and low potent cannabis preparations in that regard. In an epidemiological study, Di Forti and colleagues [72] investigated 280 cases presenting with a first episode of psychosis and 174 controls to study the differential effects of high versus low potency cannabis. It was revealed that, among those who used cannabis, first-episode patients were almost seven times more likely to have used high potency cannabis than the controls, independent of confounding factors. Additionally, there was evidence for a dose-response relationship as those who used high-potency cannabis daily were six times more likely to be in the patient group compared to those who used it less frequently [72]. The link between patient status and high-potency cannabis might imply that patients have a preference for stronger cannabis preparations. On the other hand, these findings emphasize that the psychosis-inducing effects of cannabis are primarily attributable to and dependent on the concentration of THC. A study by Morgan and colleagues, who analyzed hair samples of 140 healthy individuals with regard to traceable levels of THC and CBD, confirmed this link: The group of individuals who had only THC in hair showed the highest levels of psychotic experiences compared to individuals with THC and CBD in hair and those without any cannabinoids in hair [70]. In a set of subsequent naturalistic studies, in which healthy participants were tested while intoxicated with their own chosen cannabis, the researchers further revealed that cannabidiol 
antagonized the negative effects of THC on cognition, both acutely and longer term $[71,73]$, and attenuated the incentive salience of cannabis-associated cues, including self-rated indices of liking of the drug [74]. Similar evidence stems from imaging studies documenting opposite effects of THC and CBD on both brain function and behavioral parameters in healthy individuals $[75,76]$.

In agreement with the above, CBD has been discussed as an antipsychotic drug [77, 78], but might also be of relevance for the treatment of drug addiction [79]. Future research should therefore focus on the identification of mechanisms of action of both endogenous cannabinoids and exogenous cannabinoids such as THC and CBD to advance our understanding of the etiology as well as treatment of schizophrenia and co-morbid cannabis abuse.

\section{Clinical relevance}

Notably, the prevalence of cannabis use is much higher among patients with psychotic disorder compared to the general population [4-6]. Research on the reasons and motives of cannabis use has shown that patients with psychotic disorder and healthy controls share similar intentions [80, 81]. Both groups most often report using cannabis to get 'high', to enhance mood and relieve dysphoria, and for reasons of social participation and relaxation. Yet patients with psychotic disorder are more likely to also use cannabis out of boredom and to avoid social isolation [82]. Studies on the effects of cannabis use in daily life are scarce, in particular in patients with psychotic symptoms. Accordingly, much on this topic remains unclear, including the question of why patients are particularly prone to continue to use cannabis. A recent study on the patterns of cannabis use in psychosis employed the Experience Sampling Method in a group of patients and revealed both a positive mood enhancing effect of cannabis and a negative hallucinogenic effect [83]. Notably, while the positive effects on mood occurred quite immediately, the negative effect on psychotic symptoms emerged only sub-acute [83]. The authors concluded that this bi-phasic pattern of cannabis effects in psychosis might be indicative of a vicious circle: The positive effects are directly linked to the use of cannabis, while the delayed experience of symptom worsening is not associated with drug taking anymore and instead fosters further use [83]. In an attempt to replicate the bi-phasic effect of cannabis in psychosis, a second cohort of patients was recently investigated applying the same technique [84]. Again, cannabis had a positive effect on mood and a negative effect on hallucinations, yet the effects cooccurred acutely and could not be temporally disentangled [84].

Despite these initial attempts to shed light on patterns of cannabis use in patients with psychosis, this issue remains in need for investigation, especially with regard to mechanisms of continuation of cannabis use in this vulnerable group. Therefore, 
another aim of this thesis was to further investigate factors that contribute to maintenance of use in psychosis (chapter 8). A central feature of drug-using behavior is craving, the strong urge or desire for the drug, also called wanting [85]. Chapter 8 accordingly investigated craving in relation to cannabis use in a group of patients with psychotic disorder and healthy controls. An earlier study, employing the cross-sectional Obsessive Compulsive Drug Use Scale (OCDUS) for cannabis use, had suggested that craving levels are increased in patients with psychotic disorder [86]. In line with this, our study also revealed higher levels of craving in patients with psychosis compared to healthy controls independent of frequency of use, when measured with the OCDUS. When measured with the Experience Sampling Method in daily life however, no differences emerged with regard to mean levels of craving intensity or frequency during the last week. Yet, craving measured with ESM in daily life better predicted daily life cannabis use compared to the OCDUS, implying that craving is assessed most accurately when measured in the moment in daily life. Moreover, although craving in daily life was significantly associated with cannabis use in daily life in both groups, this association was stronger in the controls than the patients, possibly implying that patients are prevented from use more often, for example due to lack of money or limited accessibility. Alternatively, patients might be using cannabis widely independent from inner cues such as the experience of craving, implying that use has become rather habit-like. This might be supported by the observation that patients often use cannabis to fight boredom [82]. Previously it had been suggested that craving levels might differ according to type of antipsychotic treatment - tight binding agents at D2 receptors were found to be associated with higher levels of craving compared to agents with a low binding profile [87]. In our study, no such differences were revealed, which is in line with one earlier study indicating no effects of olanzapine or risperidone on craving for cannabis in schizophrenia [88]. Yet, numbers of individuals per treatment group in our study were low. Thus, failure to detect differences could also be due to a lack of power. Since Dekker and colleagues [86], who were the first to indicate higher levels of craving in psychosis patients, did not include any statistical adjustment for the effects of medication, this issue needs further clarification.

Overall, cannabis use has been associated with poorer outcome and higher relapse rates in patients with psychotic illness [89], while cessation of use after a first episode of psychosis has been shown to have beneficial effects on mental health outcomes [90]. Accordingly, cannabis use constitutes a major concern in the treatment of co-morbid patients and research has put effort in the development and evaluation of effective treatment strategies [91]. Cognitive Behavioral Therapy in combination with motivational interviewing had been suggested to be of particular promise [91, 92]. However, a recently evaluated trial in patients with psychotic disorder and co-morbid substance use indicated only little improvement in terms of a 
reduction in the amount of substance used per day [93]. No improvement was observed with regard to overall frequency of use or in relation to any form of clinical or functional outcome or measures of psychopathology [93]. Together with our findings discussed in chapter 8 of this thesis, these observations emphasize the need to further elucidate patterns of cannabis use in patients with psychotic disorder.

\section{Conclusion}

Overall, the research presented in this thesis has contributed towards a better understanding of the mechanisms behind the association between exposure to cannabis and an increased risk of developing psychotic symptoms and schizophrenia, both from an epidemiological perspective and with regard to neurobiological underpinnings. Evidence could be strengthened that cannabis use precedes the onset of psychosis and negatively impacts on the persistence of subclinical psychotic symptoms. This thesis moreover provided evidence for environmental moderation of the psychosis-inducing effects of cannabis, in form of interaction between urbancity and cannabis use. With regard to the neurobiological mechanism by which exposure to cannabis might increase psychosis risk, the research presented in this thesis postulates an important, although clearly not exclusive, role for the dopamine system. Finally, it was shown that craving for cannabis, which might be most accurately assessed in the moment in daily life, is strongly associated with cannabis use and might thus play a central role in maintaining drug use.

Future research should be dedicated to shedding further light on the involvement of the endocannabinoid system in psychotic disorders as well as in drug-addiction. Especially the involvement of the $C B 1$ receptor in the mediation of the effects of cannabis on the brain is in urgent need of further investigation. Moreover, the mechanisms of action of cannabinoids other than THC, such as CBD, remain widely unknown and knowledge on how CBD brings about its potentially antipsychotic effects would greatly advance the development of novel pharmacological treatment strategies. Finally, it is of major importance to gain better insight into patterns of initiation and continuation of use in vulnerable populations such as patients with psychotic symptoms, in particular with regard to the influence of antipsychotic medication on levels of craving for cannabis. Together, such research advances will hopefully translate into prevention and treatment strategies that will not only improve quality of life of many patients but also help to inform adolescents towards a sensible dealing with cannabis. 


\section{References}

1. Moore, T.H., et al., Cannabis use and risk of psychotic or affective mental health outcomes: a systematic review. Lancet, 2007. 370(9584): p. 319-28.

2. Henquet, C., et al., The environment and schizophrenia: the role of cannabis use. Schizophrenia Bulletin, 2005. 31(3): p. 608-12.

3. McGrath, J., et al., Association between cannabis use and psychosis-related outcomes using sibling pair analysis in a cohort of young adults. Archives of General Psychiatry, 2010. 67(5): p. 440-7.

4. Green, B., R. Young, and D. Kavanagh, Cannabis use and misuse prevalence among people with psychosis. Br J Psychiatry, 2005. 187: p. 306-13.

5. Gregg, L., C. Barrowclough, and G. Haddock, Reasons for increased substance use in psychosis. Clinical Psychology Review, 2007. 27(4): p. 494-510.

6. Koskinen, J., et al., Rate of cannabis use disorders in clinical samples of patients with schizophrenia: a meta-analysis. Schizophrenia Bulletin, 2010. 36(6): p. $1115-30$.

7. Verdoux, H. and M. Tournier, Cannabis use and risk of psychosis: an etiological link? Epidemio Psichiatr Soc, 2004. 13(2): p. $113-9$.

8. Minozzi, S., et al., An overview of systematic reviews on cannabis and psychosis: discussing apparently conflicting results. Drug Alcohol Review, 2010. 29(3): p. 304-17.

9. Kuepper, R., et al., Continued cannabis use and risk of incidence and persistence of psychotic symptoms: 10 year follow-up cohort study. British Medical Journal, 2011 1. 342: p. d738.

10. Henquet, C., et al., Prospective cohort study of cannabis use, predisposition for psychosis, and psychotic symptoms in young people. British Medical Journal, 2005. 330(7481): p. 11

11. Goswami, S., et al., Substance-abusing schizophrenics: do they self-medicate? American Journal of Addiction, 2004. 13(2): p. 139-50.

12. Arendt, M., et al., Cannabis-induced psychosis and subsequent schizophrenia-spectrum disorders: follow-up study of 535 incident cases. British Journal of Psychiatry, 2005. 187: p. 510-5.

13. Large, M., et al., Cannabis use and earlier onset of psychosis: a systematic meta-analysis. Arch Gen Psychiatry, $2011.68(6):$ p. 555-61.

14. van Os, J., et al., A systematic review and meta-analysis of the psychosis continuum: evidence for a psychosis proneness-persistence-impairment model of psychotic disorder. Psychological Medicine, 2009. 39(2): p. 179-95.

15. Weiser, M. and S. Noy, Interpreting the association between cannabis use and increased risk for schizophrenia. Dialogues Clin Neurosci, 2005. 7(1): p. 81-5.

16. Henquet, C., et al., Gene-environment interplay between cannabis and psychosis. Schizophrenia Bulletin, 2008. 34(6): p. $1111-21$.

17. van Winkel, R., Family-Based Analysis of Genetic Variation Underlying Psychosis-Inducing Effects of Cannabis: Sibling Analysis and Proband Follow-up. Archives of General Psychiatry, 2011. 68(2): p. 148-57.

18. G.R.O.U.P., Evidence that familial liability for psychosis is expressed as differential sensitivity to cannabis: an analysis of patient-sibling and sibling-control pairs. Archives of General Psychiatry, $2011.68(2):$ p. 138-47.

19. Caspi, A., et al., Moderation of the effect of adolescent-onset cannabis use on adult psychosis by a functional polymorphism in the catechol-O-methyltransferase gene: longitudinal evidence of a gene $X$ environment interaction. Biological Psychiatry, 2005. 57(10): p. 1117-27.

20. Henquet, C., et al., An experimental study of catechol-o-methyltransferase Val158Met moderation of delta-9-tetrahydrocannabinol-induced effects on psychosis and cognition. Neuropsychopharmacology, 2006. 31 (12): p. 2748-57.

21. Krabbendam, L. and J. van Os, Schizophrenia and urbanicity: a major environmental influence-conditional on genetic risk. Schizophrenia Bulletin, 2005. 31 (4): p. 795-9. 
22. Harley, M., et al., Cannabis use and childhood trauma interact additively to increase the risk of psychotic symptoms in adolescence. Psychological Medicine, 2010. 40(10): p. 1627-34.

23. Houston, J.E., et al., Childhood sexual abuse, early cannabis use, and psychosis: testing an interaction model based on the National Comorbidity Survey. Schizophrenia Bulletin, 2008. 34(3): p. 580-5.

24. Konings, M., et al., Replication in two independent population-based samples that childhood maltreatment and cannabis use synergistically impact on psychosis risk. Psychol Med, 201 1: p. 1-1 1.

25. Kuepper, R., et al., Do cannabis and urbanicity co-participate in causing psychosis? Evidence from a 10-year follow-up cohort study. Psychological Medicine, 201 1: p. 1-9.

26. Kuepper, R., et al., Non-replication of interaction between cannabis use and trauma in predicting psychosis. Schizophr Res, 2011 . 131(1-3): p. 262-3.

27. Hanssen, M., et al., The incidence and outcome of subclinical psychotic experiences in the general population. British Journal of Clinical Psychology, 2005. 44(P+ 2): p. $181-91$.

28. Dominguez, M.D., et al., Evidence that onset of clinical psychosis is an outcome of progressively more persistent subclinical psychotic experiences: an 8-year cohort study. Schizophrenia Bulletin, 2011. 37(1): p. 84-93.

29. Mackie, C.J., N. Castellanos-Ryan, and P.J. Conrod, Developmental trajectories of psychotic-like experiences across adolescence: impact of victimization and substance use. Psychological Medicine, $2011.41(1):$ p. $47-58$.

30. Cougnard, A., et al., Does normal developmental expression of psychosis combine with environmental risk to cause persistence of psychosis? A psychosis proneness-persistence model. Psychological Medicine, 2007. 37(4): p. 513-27.

31. van Os, J., et al., The schizophrenia envirome. Curr Opin Psychiatry, 2005. 18(2): p. $141-5$.

32. Collip, D., I. Myin-Germeys, and J. Van Os, Does the concept of "sensitization" provide a plausible mechanism for the putative link between the environment and schizophrenia? Schizophrenia Bulletin, 2008. 34(2): p. 220-5.

33. Lataster, J., et al., Adversity and psychosis: a 10-year prospective study investigating synergism between early and recent adversity in psychosis. Acta Psychiatr Scand, 2011.

34. Myin-Germeys, I., P. Delespaul, and J. van Os, Behavioural sensitization to daily life stress in psychosis. Psychological Medicine, 2005. 35(5): p. 733-41.

35. van Winkel, R., N.C. Stefanis, and I. Myin-Germeys, Psychosocial stress and psychosis. A review of the neurobiological mechanisms and the evidence for gene-stress interaction. Schizophrenia Bulletin, 2008. 34(6): p. 1095-105.

36. Lardinois, M., et al., Childhood trauma and increased stress sensitivity in psychosis. Acta Psychiatrica Scandinavica, 2011 . 123(1): p. 28-35.

37. Boileau, I., et al., Modeling sensitization to stimulants in humans: an [1 1C]raclopride/positron emission tomography study in healthy men. Archives of General Psychiatry, 2006. 63(12): p. 1386-95.

38. Lodge, D.J. and A.A. Grace, Amphetamine activation of hippocampal drive of mesolimbic dopamine neurons: a mechanism of behavioral sensitization. J Neurosci, 2008. 28(31): p. 7876-82.

39. Chen, J.C., et al., Significance of glutamate and dopamine neurons in the ventral pallidum in the expression of behavioral sensitization to amphetamine. Life Sci, 2001. 68(9): p. 973-83.

40. Patrick, S.L., et al., Concomitant sensitization of amphetamine-induced behavioral stimulation and in vivo dopamine release from rat caudate nucleus. Brain Res, 1991. 538(2): p. 343-6.

41. Robinson, T.E. and J.B. Becker, Behavioral sensitization is accompanied by an enhancement in amphetamine-stimulated dopamine release from striatal tissue in vitro. European Journal of Pharmacology, 1982. 85(2): p. 253-4.

42. Cadoni, C., V. Valentini, and G. Di Chiara, Behavioral sensitization to delta 9-tetrahydrocannabinol and cross-sensitization with morphine: differential changes in accumbal shell and core dopamine transmission. Journal of Neurochemistry, 2008. 106(4): p. 1586-93.

43. Kolb, B., et al., Chronic treatment with Delta-9-tetrahydrocannabinol alters the structure of neurons in the nucleus accumbens shell and medial prefrontal cortex of rats. Synapse, 2006. 60(6): p. 429-36. 
44. Kuepper, R., et al., Does dopamine mediate the psychosis-inducing effects of cannabis? A review and integration of findings across disciplines. Schizophr Res, 2010. 121(1-3): p. 107-17.

45. Bossong, M.G., et al., Delta9-Tetrahydrocannabinol Induces Dopamine Release in the Human Striatum. Neuropsychopharmacology, 2008.

46. Stokes, P.R., et al., Can recreational doses of THC produce significant dopamine release in the human striatum? Neuroimage, 2009. 48(1): p. 186-90.

47. Barkus, E., et al., Does intravenous \{triangleup\}9-tetrahydrocannabinol increase dopamine release? A SPET study. J Psychopharmacol, 2010.

48. D'Souza, D.C., et al., Delta-9-tetrahydrocannabinol effects in schizophrenia: implications for cognition, psychosis, and addiction. Biological Psychiatry, 2005. 57(6): p. 594-608.

49. D'Souza, D.C., et al., Effects of haloperidol on the behavioral, subjective, cognitive, motor, and neuroendocrine effects of Delta-9-tetrahydrocannabinol in humans. Psychopharmacology (Berl), 2008. 198(4): p. 587-603.

50. Morrison, P.D. and R.M. Murray, From real-world events to psychosis: the emerging neuropharmacology of delusions. Schizophrenia Bulletin, 2009. 35(4): p. 668-74.

51. Konings, M., et al., Early exposure to cannabis and risk for psychosis in young adolescents in Trinidad. Acta Psychiatrica Scandinavica, 2008. 118 (3): p. 209-13.

52. Arseneault, L., et al., Cannabis use in adolescence and risk for adult psychosis: longitudinal prospective study. British Medical Journal, 2002. 325(7374): p. 1212-3.

53. Schneider, M., E. Schomig, and F.M. Leweke, Acute and chronic cannabinoid treatment differentially affects recognition memory and social behavior in pubertal and adult rats. Addict Biol, 2008. 13(3-4): p. $345-57$.

54. Viveros, M.P., et al., The endocannabinoid system in critical neurodevelopmental periods: sex differences and neuropsychiatric implications. J Psychopharmacol, 2011.

55. Hashimotodani, Y., T. Ohno-Shosaku, and M. Kano, Endocannabinoids and synaptic function in the CNS. The Neuroscientist, 2007. 13: p. 127-137.

56. Bossong, M.G. and R.J. Niesink, Adolescent brain maturation, the endogenous cannabinoid system and the neurobiology of cannabis-induced schizophrenia. Progress in Neurobiology, 2010.

57. Leweke, M., et al., Elevated endogenous cannabinoids in schizophrenia. Neuroreport, 1999. 10(8): p. 1665-9.

58. Giuffrida, A., et al., Cerebrospinal anandamide levels are elevated in acute schizophrenia and are inversely correlated with psychotic symptoms. Neuropsychopharmacology, 2004. 29(1 1): p. 2108-14.

59. Koethe, D., et al., Anandamide elevation in cerebrospinal fluid in initial prodromal states of psychosis. Br J Psychiatry, 2009. 194(4): p. 371-2.

60. Wong, D.F., et al., Quantification of cerebral cannabinoid receptors subtype 1 (CB1) in healthy subjects and schizophrenia by the novel PET radioligand [1 1C]OMAR. Neuroimage, 2010. 52(4): $p$ 1505-13.

61. Zavitsanou, K., T. Garrick, and X.F. Huang, Selective antagonist [3H]SR141716A binding to cannabinoid $C B 1$ receptors is increased in the anterior cingulate cortex in schizophrenia. Prog Neuropsychopharmacol Biol Psychiatry, 2004. 28(2): p. 355-60.

62. Ujike, H. and Y. Morita, New perspectives in the studies on endocannabinoid and cannabis: cannabinoid receptors and schizophrenia. J Pharmacol Sci, 2004. 96(4): p. 376-81

63. Newell, K.A., C. Deng, and X.F. Huang, Increased cannabinoid receptor density in the posterior cingulate cortex in schizophrenia. Exp Brain Res, 2006. 172(4): p. 556-60.

64. Koethe, D., et al., Expression of $C B 1$ cannabinoid receptor in the anterior cingulate cortex in schizophrenia, bipolar disorder, and major depression. J Neural Transm, 2007. 114(8): p. 1055-63.

65. Deng, C., M. Han, and X.F. Huang, No changes in densities of cannabinoid receptors in the superior temporal gyrus in schizophrenia. Neurosci Bull, 2007. 23(6): p. 341-7. 
66. Rodriguez de Fonseca, F., et al., Downregulation of rat brain cannabinoid binding sites after chronic delta 9-tetrahydrocannabinol treatment. Pharmacol Biochem Behav, 1994. 47(1): p. 33-40.

67. Leweke, M. and D. Koethe, Cannabis and psychiatric disorders: it is not only addiction. Addiction Biology, 2008. 13(2): p. 264-75.

68. Potter, D.J., P. Clark, and M.B. Brown, Potency of delta 9-THC and other cannabinoids in cannabis in England in 2005: implications for psychoactivity and pharmacology. J Forensic Sci, 2008. 53(1): p. 90-4.

69. Leweke, F., et al., Antipsychotic effects of cannabidiol. European Psychiatry, 2009. 24(suppl 1): p. 207.

70. Morgan, C.J. and H.V. Curran, Effects of cannabidiol on schizophrenia-like symptoms in people who use cannabis. British Journal of Psychiatry, 2008. 192(4): p. 306-7.

71. Morgan, C.J., et al., Impact of cannabidiol on the acute memory and psychotomimetic effects of smoked cannabis: naturalistic study: naturalistic study [corrected]. British Journal of Psychiatry, 2010. 197(4): p. 285-90.

72. Di Forti, M., et al., High-potency cannabis and the risk of psychosis. British Journal of Psychiatry, 2009. 195(6): p. 488-91.

73. Morgan, C.J., et al., Sub-chronic impact of cannabinoids in street cannabis on cognition, psychotic-like symptoms and psychological well-being. Psychol Med, 2011: p. 1-10.

74. Morgan, C.J., et al., Cannabidiol attenuates the appetitive effects of Delta 9-tetrahydrocannabinol in humans smoking their chosen cannabis. Neuropsychopharmacology, 2010. 35(9): p. 1879-85.

75. Bhattacharyya, S., et al., Opposite Effects of Delta-9-Tetrahydrocannabinol and Cannabidiol on Human Brain Function and Psychopathology. Neuropsychopharmacology, 2009.

76. Fusar-Poli, P., et al., Distinct effects of \{delta\}9-tetrahydrocannabinol and cannabidiol on neural activation during emotional processing. Archives of General Psychiatry, 2009. 66(1): p. 95-105.

77. Zuardi, A.W., et al., Cannabidiol monotherapy for treatment-resistant schizophrenia. J Psychopharmacol, 2006. 20(5): p. 683-6.

78. Zuardi, A.W., et al., Cannabidiol, a Cannabis sativa constituent, as an antipsychotic drug. Braz J Med Biol Res, 2006. 39(4): p. 421-9.

79. Maldonado, R., O. Valverde, and F. Berrendero, Involvement of the endocannabinoid system in drug addiction. Trends Neurosci, 2006. 29(4): p. 225-32.

80. Dekker, N., D.H. Linszen, and L. De Haan, Reasons for cannabis use and effects of cannabis use as reported by patients with psychotic disorders. Psychopathology, 2009. 42(6): p. 350-60.

81. Kolliakou, A., et al., Why do patients with psychosis use cannabis and are they ready to change their use? Int J Dev Neurosci, 2011 1. 29(3): p. 335-46.

82. Schaub, M., K. Fanghaenel, and R. Stohler, Reasons for cannabis use: patients with schizophrenia versus matched healthy controls. Aust N Z J Psychiatry, 2008. 42(12): p. 1060-5.

83. Henquet, C., et al., Psychosis reactivity to cannabis use in daily life: an experience sampling study. $\mathrm{Br} \mathrm{J}$ Psychiatry, 2010. 196(6): p. 447-53.

84. Oorschot, M., et al., Predictors and consequences of cannabis use in daily life of patients with psychotic disorder. submitted.

85. Robinson, T.E. and K.C. Berridge, The neural basis of drug craving: an incentive-sensitization theory of addiction. Brain Res Brain Res Rev, 1993. 18(3): p. 247-91.

86. Dekker, N., et al., Craving for cannabis in patients with psychotic disorderd, their non-affected siblings and healthy controls: psychometric analysis of the Obsessive Compulsive Drug Use Scale. International Journal of Methods in Psychiatric Research, in press.

87. Machielsen, M., et al., Differences in craving for cannabis between schizophrenia patients using risperidone, olanzapine or clozapine. J Psychopharmacol, 2011. 
88. van Nimwegen, L.J., et al., Effect of olanzapine and risperidone on subjective well-being and craving for cannabis in patients with schizophrenia or related disorders: a double-blind randomized controlled trial. Can J Psychiatry, 2008. 53(6): p. 400-5.

89. Zammit, S., et al., Effects of cannabis use on outcomes of psychotic disorders: systematic review. British Journal of Psychiatry, 2008. 193(5): p. 357-63.

90. Gonzalez-Pinto, A., et al., Cannabis and first-episode psychosis: different long-term outcomes depending on continued or discontinued use. Schizophr Bull, 2011 . 37(3): p. 631-9.

91. Baker, A.L., L. Hides, and D.I. Lubman, Treatment of cannabis use among people with psychotic or depressive disorders: a systematic review. J Clin Psychiatry, 2010. 71(3): p. 247-54.

92. Di Forti, M., et al., Cannabis use and psychiatric and cogitive disorders: the chicken or the egg? Current Opinion in Psychiatry, 2007. 20(3): p. 228-34.

93. Barrowclough, C., et al., Integrated motivational interviewing and cognitive behavioural therapy for people with psychosis and comorbid substance misuse: randomised controlled trial. British Medical Journal, 2010. 341 : p. c6325. 

SUMMARY

ACKNOWLEDGMENTS

CURRICULUM VITAE

Publications 
190 | SUMMARY 


\section{SUMMARY}

The use of cannabis has been widely established as a risk factor for psychosis. Yet, the exact nature of the association still remains unclear, including the issue of selfmedication or reverse causality. Previously it had been suggested that the link between cannabis and psychosis might best be understood in terms of interaction between genes and environment, where individual vulnerability combines with environmental risk such as cannabis use in causing psychotic disorder. However, it is widely unknown whether cannabis use might also interact with other environmental risk factors. In addition, the biological mechanisms behind such findings remain elusive.

This thesis therefore considered epidemiological (studied in chapters 2, 3 and 4 ) as well as biological mechanisms (discussed in chapters 5, 6, 7, and 9) that may underlie the association between exposure to cannabis and the development of psychotic symptoms and schizophrenia.

CHAPTER 1 provides an overview on the epidemiology and etiology of psychotic disorders such as schizophrenia and discusses cannabis use as an important environmental risk factor. Furthermore, epidemiological studies on the link between cannabis use and psychosis are reviewed and an outlook is given on potential biological correlates of this association, concluding that dopamine might play a role in mediating the effects of cannabis on psychosis risk. The chapter subsequently discusses existing findings on differential sensitivity to the effects of cannabis, indicating that genetic predisposition for psychosis as well as variation in specific molecular polymorphisms might give rise to increased vulnerability. Also, cannabidiol is introduced as another cannabinoid and ingredient of cannabis with potential antipsychotic properties. Finally, research gaps in the study of cannabis as cause of psychosis are identified and the aims of the current thesis are outlined.

CHAPTER 2 deals with the temporal association between exposure to cannabis and the development of psychotic symptoms to shed further light on the nature of this association. In addition it was of interest whether cannabis use increases psychosis risk by impacting on the persistence of subclinical psychotic symptoms. For this purpose, data from a large German prospective cohort study of adolescents and young adults (Early Developmental Stages of Psychopathology, EDSP) were analyzed. The following research questions were formulated: i) Does cannabis use precede the onset of incident subclinical psychotic symptoms in adolescence and young adults? And ii) Does cannabis use increase psychosis risk by negatively impacting on the persistence of these subclinical psychotic symptoms? Analyses of the 
data revealed that, in individuals who were cannabis naïve at baseline, use of cannabis between baseline and first follow-up was significantly associated with increased risk of incident psychotic symptoms at second follow-up. Moreover it was revealed that continued use of cannabis (i.e. cannabis use at both baseline and first follow-up) increased the risk that subclinical psychotic symptoms persisted throughout the period between first and second follow-up. Together these findings provide further evidence for a causal role of cannabis use in the etiology of psychosis. Moreover, a mechanism of abnormal persistence of subclinical psychotic symptoms is suggested to form part of the pathway from cannabis to psychosis.

CHAPTER 3 considers the possibility of environmental moderation of the cannabispsychosis relationship. In particular we investigated whether urbanicity - growing up in an urban environment - played a role for the long-term psychosis-inducing effects of cannabis. Again, data from the German EDSP study were analyzed and the interaction between urbanicity and cannabis use at follow-up was calculated. To ensure the prediction of incident psychotic symptoms, all individuals with preexisting psychotic symptoms at baseline were excluded from the analyses. It was revealed that the risk to develop psychotic symptoms following cannabis use was much higher for individuals who grew up in an urban environment compared to individuals from the rural surroundings. This suggests that, in addition to genetic factors, exposure to environmental risk factors may induce increased sensitivity to the psychosis-inducing effects of cannabis.

CHAPTER 4 presents a further epidemiological study on environmental moderation of the cannabis-psychosis relationship. Previous research had indicated that trauma interacts with cannabis use in causing psychosis: Individuals who were exposed to trauma during childhood had a higher risk of developing psychotic symptoms and schizophrenia following cannabis use later in life. The present study aimed at replication of this finding in the large cohort of the prospective German EDSP study. Contrary to our hypothesis and to previous findings, trauma was not associated with increased risk of developing psychotic symptoms after cannabis use.

CHAPTER 5 provides an update on the dopamine dysfunction in psychosis and schizophrenia. This includes neurochemical imaging studies on mechanisms of presynaptic and postsynaptic dopamine function in both striatal and extrastriatal regions of the brain, including prefrontal areas. It is concluded that contrary to the long-standing claim of involvement of mesolimbic dopamine dysregulation, it is rather a dysfunction in nigrostriatal dopaminergic pathways and in particular associative striatum that is associated with (early) expression of symptomatology in psychosis. 
In order to elucidate the role of dopamine in the psychosis inducing effects of cannabis, CHAPTER 6 summarizes and integrates research across different disciplines on the interaction between the dopamine and the endocannabinoid system. Dopamine plays a central role in the emergence as well as experience of psychotic symptoms. Striatal dopaminergic hyperfunction has been implicated in the positive symptoms of psychosis, while dopaminergic hypoactivity in prefrontal brain regions has been assumed to underlie the expression of negative symptoms. Evidence from animal research suggests that endocannabinoids are key components in the regulation of dopaminergic neurotransmission, both in striatal and prefrontal brain regions. Moreover, THC has been shown to differentially affect these pathways. It is concluded that the repeated administration of THC might alter PFC function and impair cognition by acting on dopamine signaling via activation of $C B 1$ receptors. In the ventral tegmental area, it seems that THC leads to burst firing of dopamine neurons and as a consequence increased dopamine levels in the striatum. Here, THC might additionally exert its effects by directly influencing synaptic plasticity. Yet, most of the evidence discussed in this chapter stems from animal research, and research into the processes underlying cannabis-induced psychosis in the human brain is demanded.

In line with the considerations put forward in chapter 6, CHAPTER 7 tests the hypothesis that THC increases the risk of developing psychotic symptoms by stimulating striatal dopamine neurotransmission. For this purpose, Positron Emission Tomography and $\left[{ }^{18} \mathrm{~F}\right]$ fallypride was used to study ligand displacement at D2 receptors - indicative of increased dopamine release - following pulmonary administration of THC in 9 patients with psychotic disorder, 9 first-degree relatives and 10 healthy controls. Analyses revealed significant dopamine release associated with administration of THC in both patients with psychotic disorder and first-degree relatives. In line with previous findings, no THC-induced dopamine release was found in healthy controls. These findings support a dopaminergic mechanism of cannabisinduced psychosis in individuals at risk for psychosis.

CHAPTER 8 investigates patterns of cannabis use in relation to craving for cannabis in daily life of patients with psychotic disorder and healthy controls. The study makes use of the Experience Sampling Method (ESM), a structured diary technique that pseudo-randomly collects multiple observations per person during several consecutive days. Participants received a digital wrist watch and a paper-and-pencil ESM booklet. Twelve times a day on six consecutive days, the watch emitted a beep at random moments. After each beep, participants were asked to fill in a selfassessment form, collecting reports on craving intensity, severity of symptoms and cannabis use rated on 7-point Likert scales at the moment of the beep. In addition to collecting information on craving levels by means of the ESM booklets, craving was 
assessed with the Obsessive Compulsive Drug Use Scale (OCDUS) for cannabis use. It was revealed that patients with psychotic disorder displayed higher levels of craving when measured with the OCDUS, but did not differ from healthy controls in craving intensity when measured in daily life with ESM. Also ESM craving was a better predictor of cannabis use during the ESM week than scores on the OCDUS. Craving in daily life was significantly associated with cannabis use in daily life. Notably, this association was stronger for controls than for patients, suggesting that patients are either prevented more often from use or alternatively use cannabis rather independent from inner cues. In general, no differences were found between patients receiving different types of antipsychotics, yet failure to detect such differences might be due to a lack of power. Overall, the study emphasizes the need to further elucidate the role of craving in relation to cannabis use in patients with psychosis, in particular with regard to the influence of antipsychotic medication. Better insight into mechanisms of cannabis use might ultimately guide the development of effective treatment strategies.

CHAPTER 9 presents an editorial addressing the issue of differences in potency of cannabis (i.e. the amount of THC) in relation to its effects on psychosis and cognition. Apart from THC, cannabis contains a range of other cannabinoids, among others cannabidiol. Notably, cannabidiol has been shown to act anxiolytic and, as suggested by studies by Morgan and colleagues, might antagonize the negative effects of THC. This is of relevance as the composition of cannabis differs widely with regard to the ratio of cannabidiol and THC. It is concluded that future research on this topic is warranted in order to shed further light on the possibly antipsychotic mechanisms of action of cannabidiol. Furthermore the importance of considering differences in the cannabidiol:THC ratio of cannabis when studying its effects on human behavior and mental health is emphasized.

Finally, CHAPTER 10 briefly summarizes the results of the studies presented in this thesis, which includes three epidemiological studies, an experimental neuroimaging, an observational study, two reviews and an editorial. The relevance of the current results is discussed on the background of existing findings. Furthermore, clinical implications are considered. Directions for future research are provided. 


\section{ACKNOWLEDGMENTS}

Leider lässt sich eine wahrhafte Dankbarkeit mit Worten nicht ausdrücken.

[Johann Wolfgang von Goethe]

First and foremost I want to thank my supervisors Cécile and Jim, whose constant personal and professional support has always encouraged me. Thanks for confiding in me.

Further, I want to thank

... all other colleagues from Mental Health, in particular Margreet, Anne, Dina, Johan and Tineke

... my colleagues from Nuclear Medicine in Maastricht and Leuven, in particular Marinus van Kroonenburgh, Emiel Beijer, Christel Demollin, Christian Urbach, Koen van Laere and Jenny Ceccarini ... the colleagues from Columbia University, in particular Anissa Abi-Dargham ... Steffen, Ruth, Sarah, Fanny, Lena, Liz und Andrea.

"You've got the love I need to see me through"

... Hannah, Silvia, Vanessa und Ruth ... Hanna, Nick, Julia und Anne ... Mama, Papa, Annika, David und Arne.

Ten slotte wil ik van harte iedereen danken die een bijdrage heeft geleverd aan onze studies, als deelnemer, onderzoeker of data-invoerder. Zonder jullie bijdrage was dit proefschrift niet mogelijk geweest. 


\section{CURriculum Vitae}

Rebecca Kuepper was born on October 9, 1979 in Aachen, Germany. In 1999 she graduated from Einhard Gymnasium Aachen, Germany. From 2002 to 2007 she studied Psychology at Maastricht University, Maastricht, the Netherlands, where she received her Bachelor degree in 2005 and her Master degree in 2007 (cum laude). During her Bachelor's she spent six month at the Universidad de Salamanca, Spain. As part of her Master's in 'Cognitive Neuroscience, Neuropsychology and Psychopathology' at Maastricht University she conducted her research internship at the Department of Psychiatry and Psychotherapy, University Medical Center Hamburg-Eppendorf, Germany, under supervision of Prof. Dr. Steffen Moritz, where she continued to work as a research assistant after her graduation. From 2008 to 2011 she worked as a PhD student at the division of Mental Health, School for Mental Health and Neuroscience (MHeNS), Department of Psychiatry and Neuropsychology at Maastricht University, the Netherlands, under supervision of Prof. Dr. Jim van Os and Dr. Cécile Henquet. In early 2011, she spent six month as a research fellow at Columbia University and New York State Psychiatric Institute, Division Translational Imaging, New York, NY, USA. She is currently working as a post-doc researcher at the Department of Psychiatry and Neuropsychology at Maastricht University. 


\section{Publications}

\section{International journals}

Konings, M., Stefanis, N., Kuepper, R., de Graaf R., ten Have, M., van Os, J., Bakoula, C., \& Henquet, C. (2012). Replication in two independent populationbased samples that childhood trauma interacts with cannabis use on psychosis risk. Psychological Medicine, 42(1), 149-159.

Kuepper, R., Henquet, C., Lieb, R., Wittchen, H.-U., \& van Os, J. (2011). Nonreplication of interaction between cannabis use and trauma in predicting psychosis. Schizophrenia Research, 131 (1-3), 262-263.

Kuepper, R., van Os, J., Lieb, R., Wittchen, H.-U., \& Henquet, C. (201 1). Do cannabis and urbanicity co-participate in causing psychosis? Evidence from a 10-year follow up cohort study. Psychological Medicine, 41(10), 2121-2129.

Kuepper, R., van Os, J., Lieb, R., Wittchen, H.-U., \& Henquet, C. (2011). Continued cannabis use and risk of incidence and persistence of psychotic symptoms: 10-year follow up cohort study, British Medical Journal, 342, d738.

Henquet, C., \& Kuepper, R. (2010). Does cannabidiol protect against the negative effects of THC? British Journal of Psychiatry, 197(4), 259-260.

Kuepper, R., Morrison, P., van Os, J., Murray, R., Kenis, G., \& Henquet, C. (2010). Does dopamine mediate the psychosis-inducing effects of cannabis? A review and integration of findings across disciplines, Schizophrenia Research, 121(1-3), 107 117.

Henquet, C., van Os, J., Kuepper, R., Smits, M., Delespaul, P., a Campo, J., \& MyinGermeys, I. (2010). Psychosis reactivity to cannabis use in daily life: an experience sampling study. British Journal of Psychiatry, 196, 447-453.

Henquet, C., Di Forti, M., Morrison, P., Kuepper, R., \& Murray, R. M. (2008). Geneenvironment interplay between cannabis and psychosis. Schizophrenia Bulletin, $34(6), 1111-1121$. 


\section{Book contributions}

Kuepper, R., Skinbjerg, M. \& Abi-Dargham, A. (in press). The Dopamine Dysfunction in Psychosis Revisited: New Insights Into Topography And Course. In Mark A. Geyer \& Gerhard Gross (Eds), Handbook of Experimental Pharmacology: Current Antipsychotics. Springer: Berlin.

Konings, M., Kuepper, R., Smits, M., \& Henquet, C. (201 1). Biologische risicofactoren: cannabisgebruik. In W. Cahn, L. Krabbendam, I. Myin-Germeys, R. Bruggeman \& L. de Haan (Eds.), Handboek Schizofrenie. Utrecht: De Tijdstroom.

\section{Submitted papers}

Kuepper, R., Ceccarini, J., Lataster, J., van Os, J., van Kroonenburgh, M., van Laere, K., Marcelis, M., \& Henquet, C. (submitted). Delta-9-tetrahydrocannabinol-induced dopamine release as a function of psychotic illness and psychotic vulnerability: [18F]fallypride PET study.

Kuepper, R.*, Oorschot, M.*, Myin-Germeys, I., van Os, J., Smits, M., \& Henquet, C. (submitted). Is craving for cannabis increased in individuals with psychotic disorder? An Experience Sampling Study.

Oorschot, M.*, Kuepper, R.*, Henquet, C., van Os, J., Smits, M., \& Myin-Germeys, I. (submitted). Predictors and consequences of cannabis use in daily life of patients with psychotic disorder: An Experience Sampling Study.

(*contributed equally)

\section{Abstracts}

Kuepper, R., van Os, J., Myin-Germeys, I., Henquet, C., \& G.R.O.U.P (2011). Geneenvironment interaction in psychosis: Effects of cannabis use, childhood trauma and genetic liability. Schizophrenia Bulletin, 37(Supplement 1), 54.

Kuepper, R., van Os, J., Lieb, R., Wittchen, H.U., \& Henquet, C. (2010). Crosssensitization between urbanicity and cannabis in psychosis. Early Intervention in Psychiatry, 4(Supplement 1), 33. 
Kuepper, R. (2010). Is het effect van cannabis op psychose sterker in de stad? Tijdschrift voor Psychiatrie, 52(Supplement 2), 99-100.

Kuepper, R., van Os, J., Lieb, R., Wittchen, H.U., \& Henquet, C. (2010). Cannabis as cause of psychosis: Effects on incidence and persistence of psychotic symptoms. Schizophrenia Research, $117(2-3), 298$.

Kuepper, R., van Os, J., Wittchen, H.U., Lieb, R., \& Henquet, C. (2009). Cannabis use and true incidence of psychotic symptoms in a population-based sample. Schizophrenia Bulletin, 35(Supplement 1), 88.

Henquet, C., Kuepper, R., Konings, M., Lieb, R., Wittchen, H.U., \& van Os, J. (2009). Do cannabis and urbanicity interact in causing psychosis? Schizophrenia Bulletin, 35(Supplement 1), 87.

Kuepper, R., Myin-Germeys, I., Konings, M., van Winkel, R., van Os, J., \& Henquet C., (2009). Exploring the causal relationship between cannabis and schizophrenia: what is the role of genes and environment? European Psychiatry, 24(Supplement 1), 31.

Kuepper, R., Klinge, R., Randjbar, S., Hottenrott, B., van Os, J., \& Moritz, S. (2007). An investigation of jumping to conclusions, need for closure and metacognitive awareness in schizophrenia patients. Der Nervenarzt, 78(Supplement 2), 96. 